\title{
A NUMERICAL STUDY OF FREE \\ CONVECTIVE HEAT TRANSFER WITHIN \\ DOMED SKYLIGHT CAVITIES
}

\author{
By \\ AmirAbbas Sartipi \\ Bachelor of Engineering (B.Eng.) \\ Azad University, Tehran, 1995
}

\author{
A thesis \\ Presented to Ryerson University \\ in partial fulfillment of the \\ requirements for the degree of \\ Master of Applied Science \\ in the Program of \\ Mechanical Engineering
}

Toronto, Ontario, Canada, 2007

(C) AmirAbbas Sartipi 2007 


\section{AUTHOR'S DECLARATION}

I hereby declare that I am the sole author of this thesis. I authorize Ryerson University to lend this thesis to other institutions or individuals for the purpose of scholarly research.

I further authorize Ryerson University to reproduce this thesis by photocopying or by other means, in total or in part, at the request of other institutions or individuals for the purpose of scholarly research. 


\section{BORROWER}

Ryerson University requires the signature of all persons using or photocopying this thesis.

Please sign below, and give address and date. 


\section{A Numerical Study of Free Convective Heat Transfer within Domed Skylight Cavities}

\section{AmirAbbas Sartipi, M.A.Sc., 2007 \\ Department of Mechanical and Industrial Engineering, Ryerson University}

Domed skylights are important architectural design elements to deliver daylight and solar heat into buildings and connect buildings' occupants to outdoors. To increase the energy efficiency of skylighted buildings, domed skylights employ a number of glazing layers forming enclosed spaces. The latter are subject to complex buoyancy-induced convection heat transfer. Currently, existing fenestration design computer tools and building energy simulation programs do not, however, cover such skylights to quantify their energy performance when installed in buildings. This work presents a numerical study on natural laminar convection within concentric and vertically eccentric domed cavities. The edges of domed cavities are assumed adiabatic and the temperature of the interior and exterior surfaces are uniform and constant. The concentric and vertically eccentric domed cavities were studied when heated from inside and heated from outside, respectively. A commercial CFD package employing the control volume approach is used to solve the laminar convective heat transfer within the cavity. The obtained results showed steady flow for small Grashof numbers. For moderate and large Grashof numbers, depending on the gap ratio and the cases of heating from inside or outside, the flow may be steady or transient periodic with a single vortex-cell or multi vortex-cells. The Nusselt number for the case of heated from inside is greater than the case of heated from outside. The numerical results show that the changes in the gap ratio have smaller effect on Nusselt number in high profile domed skylights than lower profile domed skylights. 


\section{ACKNOWLEDGEMENTS}

The author would like to express his sincerest appreciation to Dr. Abdelaziz (Aziz) Laouadi and Dr. David Naylor. Their invaluable input and guidance led to the successful completion of this thesis. Their knowledge and enthusiasm provided ongoing motivation, and a sense of greater responsibility as an engineer. Also, the author would like to thank Dr. Ramdhane Dhib for his valuable input and financial support.

The author would also like to acknowledge the financial support of the National Research Council of Canada, the Panel for Energy Research and Development (PERD), B.C. Hydro and Natural Resources Canada.

The author wishes to thank his family, specially his sister Yasi, who has given ongoing support and encouragement. Also, a special thanks to Bita for her love and support during the course of this thesis. 
Author's Declaration ii

Borrower __iii

Abstract _ iv

Acknowledgements __ $\mathrm{V}$

Table of Contents _ vi

List of Tables___

List of Figures __ xii

Nomenclature _ $\mathrm{xv}$

CHAPTER 1 - LITERATURE REVIEW __ 1

1.1 Introduction __ 1

1.2 Overview __ 2

1.3 Natural Convection Flow ___ 2

1.4 Dimensionless Numbers ___ 3

1.5 Previous Work _ 5

1.5.1 Natural Laminar Convection within Domed Cavities ___ 6

1.5.2 Natural Convection from Interior Surface of Dome $\_10$

1.5.3 Forced and Natural Convection from Exterior Surface of Dome __ 14

1.6 Conclusion__ 19

1.7 Scope of Research _ 19

1.8 Approach _ 20

CHAPTER 2 - MATHEMATICAL FORMULATION _ 21

2.1 Introduction 21

2.2 Modeling The Geometry __ 21

2.3 Assumptions _ 25 
2.3.1 Newtonian Fluid 25

2.3.2 Physical Properties and Boundary Conditions 26

2.3.3 Boussinesq Approximation and Incompressible Flow 26

2.3.4 Axisymmetric Two-Dimensional Flow 28

2.4 Governing Equations 28

2.4.1 Flow in Domed Cavities 29

2.4.2 Boundary Conditions 31

2.5 Dimensionless Governing Equations 33

2.6 Evaluation of Heat Transfer 35

2.6.1 Cavities with Uniform Gap Thickness 36

2.6.2 Cavities with Non-Uniform Gap Thickness 40

2.7 Transient Heat Transfer 41

2.8 Table of Governing Parameters 42

CHAPTER 3 - NUMERICAL APPROACH 44

3.1 Introduction 44

3.2 Numerical Method, Parameters and Order of Accuracy 45

3.3 Boundary and Initial Conditions 47

3.4 Grid Topology and Grid Density 48

3.5 Accuracy and Convergence Criteria 51

3.6 Flow Stability and Critical Rayleigh Number 52

3.7 Numerical Model Validation 53

3.8 Summary 60

4.1 Introduction 61

4.2 Results for Domed Concentric Cavities 61

4.2.1 Concentric Cavities with Truncation Angle $\left(\theta_{0}\right)$ of $90^{\circ}$ 61

4.2.1.1 Correlations for heat Transfer 72 
4.2.2 Concentric Cavities with Truncation Angle of $\left(\theta_{0}=45^{\circ}\right) \_81$

4.2.2.1 Correlations for heat Transfer _ 85

4.2.3 Concentric Cavities with Truncation Angle of $\left(\theta_{0}=30^{\circ}\right) \_92$

4.2.3.1 Correlations for heat Transfer __ 96

4.2.4 Discussion _ 102

4.3 Results for Eccentric Dome Cavities___ 105

4.3.1 Discussion _ 110

CHAPTER 5 - CONCLUSIONS AND RECOMMENDATIONS _ 114

5.1 Conclusions _ 114

5.2 Recommendations _ 116

\section{APPENDIX A- UNIFORM GAP THICKNESS DOMED SKYLIGHT HEATED} FROM INSIDE

A.1 Average Nusselt Number -118

A.2 Curve-Fitting to Coefficient $c$ and $d \_124$

A.3 Curve-Fitting to The Nusselt Number _ _ 126

APPENDIX B - NON-UNIFORM GAP THICKNESS DOMED SKYLIGHT HEATED FROM OUTSIDE

B.1 Average Nusselt Number 148

B.2 Curve-Fitting to Coefficient $c$ and $d$ 156

B.3 Curve-Fitting to the Nusselt Number 163

REFERENCES 189 
Table Title

Page

2.1 The value of the parameters 43

3.1 Comparison of Nusselt number with different grid density $\left(\theta_{0}=30^{\circ}, \mathrm{T}_{\mathrm{o}}<\mathrm{T}_{\mathrm{i}}, \delta=0.1\right.$ and $\varepsilon=0)$ 50

3.2 Nusselt number comparison between present model and Raithby and Hollands (1975) in spherical cavities $\left(\varepsilon=0, \theta_{0}=180^{\circ}, \delta=1\right.$ and $\left.\mathrm{T}_{0}<\mathrm{T}_{\mathrm{i}}\right)$ 54

3.3 Nusselt number comparison between present model and Grag (1991) in spherical cavities $\left(\varepsilon=0, \theta_{0}=180^{\circ}, \delta=1\right.$ and $\left.\mathrm{T}_{0}<\mathrm{T}_{\mathrm{i}}\right)$ 55

3.4 Nusselt number comparison between present model and Laouadi (2001) in spherical cavities $\left(\varepsilon=0, \theta_{0}=180^{\circ}, \delta=1\right.$ and $\left.\mathrm{T}_{0}<\mathrm{T}_{\mathrm{i}}\right)$ 56

3.5 Nusselt number comparison between present model and equation 3.8 $\left(\varepsilon=0, \theta_{0}=45^{\circ}, \delta=0.5\right.$ and $\left.\mathrm{T}_{0}>\mathrm{T}_{\mathrm{i}}\right)$ 58

3.6 Nusselt number comparison between present model and equation 3.8 $\left(\varepsilon=0, \theta_{0}=90^{\circ}, \delta=0.2\right.$ and $\left.\mathrm{T}_{0}>\mathrm{T}_{\mathrm{i}}\right)$

4.1 The constant $c$ and $d$ for $\theta_{0}=90^{\circ}$ 73

4.2 Nusselt number calculated from present model and power function curve-fitting for $\delta=0.4$ 75

4.3 Constant $c$ and $d$ for $\theta_{0}=90^{\circ}$ 77

4.4 The Nusselt number comparison between present numerical model and equation 4.4 for $\theta_{0}=90^{\circ} \delta=0.4$ 79

4.5 The average percentage error in Nusselt number calculated from equation 4.4 and present model for $\theta_{0}=90^{\circ}$ 80

4.6 The constant $c$ and $d$ for $\theta_{0}=45^{\circ}$ 85 
4.7 Nusselt number calculated from present model and power function curve-fitting in $\delta=0.5$

4.8 Constant $c$ and $d$ for $\theta_{0}=45^{\circ}$

4.9 The Nusselt number comparison between present model and equation 4.4 for $\theta_{\mathrm{o}}=45^{\circ} \delta=0.3$

4.10 The average percentage error in Nusselt number calculated from equation 4.4 and present model for $\theta_{0}=45^{\circ}$

4.11 The constant $c$ and $d$ for $\theta_{0}=90^{\circ}$ 97

4.12 Nusselt number calculated from present model and power function curve-fitting for $\delta=0.2$ 98

4.13 Constant $c$ and $d$ for $\theta_{\mathrm{o}}=30^{\circ}$ 100

4.14 The Nusselt number comparison between present model and equation 4.4 for $\theta_{0}=30^{\circ} \delta=0.3$ 101

4.15 The average percentage error in Nusselt number calculated from equation 4.4 and present model for $\theta_{0}=30^{\circ}$ 102

4.16 The maximum absolute error in equation 4.4 related to the numerical results 110

A.1 Average Nusselt number when $\theta_{0}=90^{\circ}$ 118

A.2 Average Nusselt number when $\theta_{0}=45^{\circ}$ 120

A.3 Average Nusselt number when $\theta_{0}=30^{\circ}$ 122

A.4 Coefficient $c$ and $d$ for $\theta_{0}=90^{\circ}$ 124

A.5 Coefficient $c$ and $d$ for $\theta_{0}=45^{\circ}$ 124

A.6 Coefficient $c$ and $d$ for $\theta_{0}=30^{\circ}$ 125 
A.7 Curve-fitting to Nusselt number and percentage difference for $\theta_{0}=90^{\circ}$

A.8 Curve-fitting to Nusselt number and percentage difference for $\theta_{0}=45^{\circ}$ 142

A.9 Curve-fitting to Nusselt number and percentage difference for $\theta_{0}=30^{\circ}$ 145

B.1 Average Nusselt number when $\theta_{0}=90^{\circ}$ 148

B.2 Average Nusselt number when $\theta_{0}=45^{\circ}$

B.3 Average Nusselt number when $\theta_{0}=30^{\circ}$ 154

B.4 Coefficient $c$ and $d$ for $\theta_{\mathrm{o}}=90^{\circ}$ 157

B.5 Coefficient $c$ and $d$ for $\theta_{\circ}=45^{\circ}$ 159

B.6 Coefficient $c$ and $d$ for $\theta_{\circ}=30^{\circ}$ 162

B.7 Curve-fitting to Nusselt number and percentage difference for $\theta_{0}=90^{\circ}$ 180

B.8 Curve-fitting to Nusselt number and percentage difference for $\theta_{0}=45^{\circ}$ 183

B.9 Curve-fitting to Nusselt number and percentage difference for $\theta_{0}=30^{\circ}$ 186 


\section{LIST OF FIGURES}

Figure Title

Page

1.1 Definition of surface angle $\zeta$ for heated and cooled surface. For $90^{\circ} \leq \zeta \leq 180^{\circ}$ the flow remains attached, while it may detach if $0^{\circ} \leq \zeta \leq 90^{\circ}$ (Raithby G.D. and Hollands K.G.T., 1998) 12

2.1 Section view of spherical caps 22

2.2 Spherical coordinates system for a dome cavity 28

2.3 Oscillation of function $\mathrm{F}(\mathrm{t})$ in time period when the time step is $\Delta \mathrm{t}$ 41

3.1 Section of a quadrilateral grid for $\theta_{0}=30^{\circ}, \varepsilon=0$ and $\delta=0.1$ 49

4.1 Streamlines and isotherms for Grashof Number of $1 \times 10^{3}\left(\theta_{0}=90^{\circ}, \varepsilon=0\right.$ and $\delta=0.8)$

4.2 Streamlines and isotherms for Grashof Number of $5 \times 10^{4}\left(\theta_{0}=90^{\circ}, \varepsilon=0\right.$ and $\delta=0.8)$

4.3 Streamlines and isotherms for Grashof Number of $5 \times 10^{6}\left(\theta_{0}=90^{\circ}, \varepsilon=0\right.$ and $\delta=0.8)$

4.4 Streamlines and isotherms for Grashof Number of $1 \times 10^{3}\left(\theta_{0}=90^{\circ}, \varepsilon=0\right.$ and $\delta=0.3$ ) 64

4.5 Streamlines and isotherms for Grashof Number of $5 \times 10^{4}\left(\theta_{0}=90^{\circ}, \varepsilon=0\right.$ and $\delta=0.3$ ) 64

4.6 Streamlines and isotherms for Grashof Number of $5 \times 10^{6}\left(\theta_{0}=90^{\circ}, \varepsilon=0\right.$ and $\delta=0.3$ )

4.7 Streamlines for Grashof Number of $5 \times 10^{4}\left(\theta_{0}=90^{\circ}, \varepsilon=0\right.$ and $\left.\delta=0.5\right)$

a) Step-increasing Grashof number b) Step-decreasing Grashof Number 66

4.8 Changes in Nusselt number in time (Time Step $=0.5 \mathrm{~s}$ ) when $\mathrm{Gr}=7.5 \times 10^{3}$ $\left(\theta_{0}=90^{\circ}, \varepsilon=0\right.$ and $\left.\delta=0.2\right)$

4.9 Isotherms and streamlines for $\delta=0.1$ when $\mathrm{Gr}=1 \times 10^{3}\left(\theta_{0}=90^{\circ}, \varepsilon=0\right)$ 68 
4.10 Isotherms and streamlines for $\delta=0.1$ when $\mathrm{Gr}=5 \times 10^{4}\left(\theta_{\mathrm{o}}=90^{\circ}, \varepsilon=0\right)$ 68

4.11 Isotherms and streamlines for $\delta=0.1$ when $\mathrm{Gr}=5 \times 10^{6}\left(\theta_{\mathrm{o}}=90^{\circ}, \varepsilon=0\right)$ 69

4.12 Changes in the Nusselt versus Rayleigh number to the power of (1/4) $\left(\theta_{\mathrm{o}}=90^{\circ}, \varepsilon=0\right.$ and $\left.\delta=0.2\right)$ 70

4.13 Oscillation in Nusselt number with the time step $=1$ and 0.1

$\left(\mathrm{Gr}=5 \times 10^{5}, \theta_{0}=90^{\circ}, \varepsilon=0\right.$ and $\left.\delta=0.01\right)$ 72

4.14 Numerically predicted Nusselt number (with $\pm 4 \%$ error bars) and power function curve-fitted to the numerical results when $\delta=0.4$ 74

4.15 The curve-fitted (equation 4.2) to the constant $c$ 76

4.16 The curve-fitted (equation 4.3) to the constant $d$ 77

4.17 Nusselt number correlation with $\pm 10 \%$ error bar and numerical results for $\theta_{\mathrm{o}}=90^{\circ}$ 80

4.18 Streamlines and isotherms for $\delta=0.8$ and $\mathrm{Gr}=5 \times 10^{4}\left(\theta_{\mathrm{o}}=45^{\circ}\right.$ and $\left.\varepsilon=0\right)$ 81

4.19 Streamlines and isotherms for $\delta=0.8$ and $\mathrm{Gr}=5 \times 10^{6}\left(\theta_{\mathrm{o}}=45^{\circ}\right.$ and $\left.\varepsilon=0\right)$ 82

4.20 Streamlines and isotherms for $\delta=0.3$ and $\mathrm{Gr}=5 \times 10^{4}\left(\theta_{\mathrm{o}}=45^{\circ}\right.$ and $\left.\varepsilon=0\right)$ 83

4.21 Streamlines and isotherms for $\delta=0.3$ and $\mathrm{Gr}=5 \times 10^{6}\left(\theta_{0}=45^{\circ}\right.$ and $\left.\varepsilon=0\right)$ 83

4.22 Streamlines and isotherms for $\delta=0.1$ and $\mathrm{Gr}=5 \times 10^{4}\left(\theta_{\mathrm{o}}=45^{\circ}\right.$ and $\left.\varepsilon=0\right)$ 84

4.23 Streamlines and isotherms for $\delta=0.1$ and $\mathrm{Gr}=5 \times 10^{6}\left(\theta_{\mathrm{o}}=45^{\circ}\right.$ and $\left.\varepsilon=0\right)$ 84

4.24 Nusselt number from numerical result ( $\pm 4 \%$ error bar) and power function fitted to the numerical results for $\delta=0.5$ 86

4.25 The curve-fitted (equation 4.6) to the constant $c$ where $0<\delta<0.3$ 88

4.26 The curve-fitted (equation 4.2) to the constant $c$ where $1 \leq \delta \leq 0.3$ 89

4.27 Nusselt number correlation with $\pm 10 \%$ error bars and numerical results for $\theta_{\mathrm{o}}=45^{\circ}$ 92

4.28 Streamlines and isotherms for $\delta=0.8$ and $\mathrm{Gr}=5 \times 10^{4}\left(\theta_{\mathrm{o}}=30^{\circ}\right.$ and $\left.\varepsilon=0\right)$ 93

4.29 Streamlines and isotherms for $\delta=0.8$ and $\mathrm{Gr}=5 \times 10^{6}\left(\theta_{\mathrm{o}}=30^{\circ}\right.$ and $\left.\varepsilon=0\right)$ 93 
4.30 Streamlines and isotherms for $\delta=0.3$ and $\mathrm{Gr}=5 \times 10^{4}\left(\theta_{0}=30^{\circ}\right.$ and $\left.\varepsilon=0\right)$ 94

4.31 Streamlines and isotherms for $\delta=0.3$ and $\mathrm{Gr}=5 \times 10^{6}\left(\theta_{0}=30^{\circ}\right.$ and $\left.\varepsilon=0\right)$ 94

4.32 Streamlines and isotherms for $\delta=0.1$ and $\mathrm{Gr}=5 \times 10^{4}\left(\theta_{0}=30^{\circ}\right.$ and $\left.\varepsilon=0\right)$ 95

4.33 Streamlines and isotherms for $\delta=0.1$ and $\mathrm{Gr}=5 \times 10^{6}\left(\theta_{0}=30^{\circ}\right.$ and $\left.\varepsilon=0\right)$ 95

4.34 Oscillation in Nusselt number when Time Step $=0.01, \delta=0.1$ and $\mathrm{Gr}=5 \times 10^{6}$ $\left(\theta_{0}=30^{\circ}\right.$ and $\left.\varepsilon=0\right)$ 96

4.35 Nusselt number from numerical result ( $\pm 4 \%$ error bar) and power function fitted to the numerical results for $\delta=0.2$ 97

4.36 The curve-fitted (equation 4.8) to the constant $c$ 99

4.37 The curve-fitted (equation 4.9) to the constant $d$ 100

4.38 Profile of the time average Nusselt number as a function of $\mathrm{Ra}^{{ }^{*} 1 / 4}$ for a truncation angle of $90^{\circ}$

4.39 Profile of the time average Nusselt number as a function of $\mathrm{Ra}^{{ }^{*}{ }^{1 / 4}}$ for a truncation angle of $45^{\circ}$ 103

4.40 Profile of the time average Nusselt number as a function of $\mathrm{Ra}^{{ }^{*}{ }^{1 / 4}}$ for a truncation angle of $30^{\circ}$ 104

4.41 Nusselt number as a function of $\delta$ 105

4.42 Streamline and isotherms for $\theta_{\mathrm{o}}=45^{\circ}$ and $90^{\circ}$ when $\mathrm{Gr}=5 \times 10^{4}$ 107

4.43 Streamline and isotherms for $\theta_{\mathrm{o}}=45^{\circ}$ and $90^{\circ}$ when $\mathrm{Gr}=5 \times 10^{6}$ 108

4.44 Profile of the time average Nusselt number as a function of $\mathrm{Ra}^{{ }^{* 1 / 4}}$ for a truncation angle of $90^{\circ}$

4.45 Profile of the time average Nusselt number as a function of $\mathrm{Ra}^{{ }^{*} / 4}$ for a truncation angle of $45^{\circ}$

4.46 Nusselt number as a function of $\delta_{\max }$ 113

A.1 Curve-fitting to the numerical results for $\theta_{\mathrm{o}}=90^{\circ}$ and $\delta=1$ 126

A.2 Curve-fitting to the numerical results for $\theta_{0}=90^{\circ}$ and $\delta=0.8$ 127 
A.3 Curve-fitting to the numerical results for $\theta_{0}=90^{\circ}$ and $\delta=0.5$

A.4 Curve-fitting to the numerical results for $\theta_{0}=90^{\circ}$ and $\delta=0.4$ 128

A.5 Curve-fitting to the numerical results for $\theta_{0}=90^{\circ}$ and $\delta=0.3$ 128

A.6 Curve-fitting to the numerical results for $\theta_{0}=90^{\circ}$ and $\delta=0.2$ 129

A.7 Curve-fitting to the numerical results for $\theta_{0}=90^{\circ}$ and $\delta=0.1$ 129

A.8 Curve-fitting to the numerical results for $\theta_{0}=90^{\circ}$ and $\delta=0.01$ 130

A.9 Curve-fitting to the numerical results for $\theta_{0}=45^{\circ}$ and $\delta=1$ 130

A.10 Curve-fitting to the numerical results for $\theta_{0}=45^{\circ}$ and $\delta=0.8$ 131

A.11 Curve-fitting to the numerical results for $\theta_{0}=45^{\circ}$ and $\delta=0.5$ 131

A.12 Curve-fitting to the numerical results for $\theta_{0}=45^{\circ}$ and $\delta=0.4$ 132

A.13 Curve-fitting to the numerical results for $\theta_{0}=45^{\circ}$ and $\delta=0.3$ 132

A.14 Curve-fitting to the numerical results for $\theta_{0}=45^{\circ}$ and $\delta=0.2$ 133

A.15 Curve-fitting to the numerical results for $\theta_{0}=45^{\circ}$ and $\delta=0.1$ 133

A.16 Curve-fitting to the numerical results for $\theta_{0}=45^{\circ}$ and $\delta=0.01$ 134

A. 17 Curve-fitting to the numerical results for $\theta_{0}=30^{\circ}$ and $\delta=1$ 134

A.18 Curve-fitting to the numerical results for $\theta_{0}=30^{\circ}$ and $\delta=0.8$ 135

A.19 Curve-fitting to the numerical results for $\theta_{0}=30^{\circ}$ and $\delta=0.5$ 135

A. 20 Curve-fitting to the numerical results for $\theta_{0}=30^{\circ}$ and $\delta=0.4$ 136

A.21 Curve-fitting to the numerical results for $\theta_{0}=30^{\circ}$ and $\delta=0.3$ 136

A.22 Curve-fitting to the numerical results for $\theta_{0}=30^{\circ}$ and $\delta=0.2$ 137

A.23 Curve-fitting to the numerical results for $\theta_{0}=30^{\circ}$ and $\delta=0.1$ 137

A.24 Curve-fitting to the numerical results for $\theta_{0}=30^{\circ}$ and $\delta=0.01$ 138 
B.1 Curve-fitting to coefficient $c$ for $\theta_{0}=90^{\circ}$ 156

B.2 Curve-fitting to coefficient $d$ for $\theta_{0}=90^{\circ}$ 156

B.3 Curve-fitting to coefficient $c$ for $\theta_{0}=45^{\circ}$ 158

B.4 Curve-fitting to coefficient $d$ for $\theta_{0}=45^{\circ}$ 158

B.5 Curve-fitting to coefficient $c$ for $\theta_{0}=30^{\circ}$ when $\delta^{\prime} \geq 0.1$ 160

B.6 Curve-fitting to coefficient $c$ for $\theta_{0}=30^{\circ}$ when $\delta^{\prime}<0.1$ 160

B.7 Curve-fitting to coefficient $d$ for $\theta_{0}=30^{\circ}$ when $\delta^{\prime}<0.1$ 161

B.8 Curve-fitting to coefficient $d$ for $\theta_{0}=30^{\circ}$ when $\delta^{\prime} \geq 0.1$ 161

B.9 Curve-fitting to the numerical results for $\theta_{0}=90^{\circ}$ and $\delta^{\prime}=0.5$ 163

B.10 Curve-fitting to the numerical results for $\theta_{0}=90^{\circ}$ and $\delta^{\prime}=0.4$ 164

B.11 Curve-fitting to the numerical results for $\theta_{0}=90^{\circ}$ and $\delta^{\prime}=0.3$ 164

B.12 Curve-fitting to the numerical results for $\theta_{0}=90^{\circ}$ and $\delta^{\prime}=0.25$ 165

B.13 Curve-fitting to the numerical results for $\theta_{0}=90^{\circ}$ and $\delta^{\prime}=0.2$ 165

B.14 Curve-fitting to the numerical results for $\theta_{0}=90^{\circ}$ and $\delta^{\prime}=0.15$ 166

B.15 Curve-fitting to the numerical results for $\theta_{0}=90^{\circ}$ and $\delta^{\prime}=0.1$ 166

B.16 Curve-fitting to the numerical results for $\theta_{0}=90^{\circ}$ and $\delta^{\prime}=0.05$ 167

B.17 Curve-fitting to the numerical results for $\theta_{0}=90^{\circ}$ and $\delta^{\prime}=0.025$ 167

B.18 Curve-fitting to the numerical results for $\theta_{0}=90^{\circ}$ and $\delta^{\prime}=0.015$ 168

B.19 Curve-fitting to the numerical results for $\theta_{0}=90^{\circ}$ and $\delta^{\prime}=0.01$ 168

B.20 Curve-fitting to the numerical results for $\theta_{0}=90^{\circ}$ and $\delta^{\prime}=0.008$ 169

B.21 Curve-fitting to the numerical results for $\theta_{0}=45^{\circ}$ and $\delta^{\prime}=0.4$ 169

B.22 Curve-fitting to the numerical results for $\theta_{0}=45^{\circ}$ and $\delta^{\prime}=0.35$ 170

B.23 Curve-fitting to the numerical results for $\theta_{0}=45^{\circ}$ and $\delta^{\prime}=0.3$ 170 
B. 24 Curve-fitting to the numerical results for $\theta_{o}=45^{\circ}$ and $\delta^{\prime}=0.25$

B.25 Curve-fitting to the numerical results for $\theta_{0}=45^{\circ}$ and $\delta^{\prime}=0.2$ 171

B.26 Curve-fitting to the numerical results for $\theta_{o}=45^{\circ}$ and $\delta^{\prime}=0.15$ 172

B.27 Curve-fitting to the numerical results for $\theta_{\mathrm{o}}=45^{\circ}$ and $\delta^{\prime}=0.1$ 172

B.28 Curve-fitting to the numerical results for $\theta_{0}=45^{\circ}$ and $\delta^{\prime}=0.05$ 173

B.29 Curve-fitting to the numerical results for $\theta_{0}=45^{\circ}$ and $\delta^{\prime}=0.025$ 173

B.30 Curve-fitting to the numerical results for $\theta_{\mathrm{o}}=45^{\circ}$ and $\delta^{\prime}=0.015$ 174

B.31 Curve-fitting to the numerical results for $\theta_{\circ}=45^{\circ}$ and $\delta^{\prime}=0.01$ 174

B.32 Curve-fitting to the numerical results for $\theta_{0}=45^{\circ}$ and $\delta^{\prime}=0.008$ 175

B.33 Curve-fitting to the numerical results for $\theta_{0}=30^{\circ}$ and $\delta^{\prime}=0.2$ 175

B.34 Curve-fitting to the numerical results for $\theta_{\circ}=30^{\circ}$ and $\delta^{\prime}=0.15$ 176

B.35 Curve-fitting to the numerical results for $\theta_{0}=30^{\circ}$ and $\delta^{\prime}=0.1$ 176

B.36 Curve-fitting to the numerical results for $\theta_{\mathrm{o}}=30^{\circ}$ and $\delta^{\prime}=0.05$ 177

B.37 Curve-fitting to the numerical results for $\theta_{0}=30^{\circ}$ and $\delta^{\prime}=0.025$ 177

B.38 Curve-fitting to the numerical results for $\theta_{0}=30^{\circ}$ and $\delta^{\prime}=0.015$ 178

B.39 Curve-fitting to the numerical results for $\theta_{\mathrm{o}}=30^{\circ}$ and $\delta^{\prime}=0.01$ 178

B.40 Curve-fitting to the numerical results for $\theta_{0}=30^{\circ}$ and $\delta^{\prime}=0.008$ 179 


\section{Symbol}

$\mathrm{A}_{\mathrm{e}}$

$\mathrm{A}_{\mathrm{n}}$

$\mathrm{A}_{\mathrm{s}}$

$\mathrm{A}_{\mathrm{w}}$

$\mathrm{A}_{\mathrm{i}}$

$\mathrm{A}_{\mathrm{o}}$

c, $d$

$\mathrm{C}_{\mathrm{p}}$

$\mathrm{Gr}$

g

$\mathrm{h}_{\mathrm{c}}$

K

$\mathrm{L}$

$\mathrm{L}_{\max }$

$\mathrm{L}^{*}{ }_{\max }$

$\mathrm{L}_{\min }$

$\mathrm{L}^{*}{ }_{\min }$

$\mathrm{Nu}_{\mathrm{i}}$

\section{Description}

: surface area of the control volume face e

: surface area of the control volume face $n$

: surface area of the control volume face s

: surface area of the control volume face $\mathrm{w}$

: surface area of the interior dome wall

: surface area of the exterior dome wall

: correlation coefficient and exponent for Nusselt number, equation

: specific heat at constant pressure

: Grashof Number, $\mathrm{Gr}=\mathrm{g} \beta\left(\mathrm{T}_{0}-\mathrm{T}_{\mathrm{i}}\right) \mathrm{L}^{3} / \mathrm{v}^{2}$

: gravitational acceleration $\left(9.81 \mathrm{~m} / \mathrm{s}^{2}\right)$

: convection film coefficient of the domed enclosure

: fluid thermal conductivity

: local gap spacing between the dome layers $\left(\mathrm{R}_{\mathrm{o}}-\mathrm{R}_{\mathrm{i}}\right)$

: maximum gap spacing between the dome layers

: dimensionless maximum gap spacing between the dome layers

$$
\left(\mathrm{L}_{\text {max }}^{*}=\mathrm{L}_{\max } / \mathrm{L}_{\max }=1\right)
$$

: minimum gap spacing between the dome layers

: dimensionless maximum gap spacing between the dome layers $\left(\mathrm{L}^{*}{ }_{\min }=\mathrm{L}_{\min } / \mathrm{L}_{\max }\right)$

: Nusselt number at the inner dome wall 


\begin{tabular}{|c|c|}
\hline $\mathrm{Nu}_{\mathrm{o}}$ & : Nusselt number at the outer dome wall \\
\hline $\mathrm{P}$ & : pressure \\
\hline $\mathrm{P}^{\prime}$ & : pressure defect $\left(\mathrm{P}^{\prime}=\mathrm{P}-\mathrm{P}_{\mathrm{c}}\right)$ \\
\hline $\mathrm{P}^{*}$ & : dimensionless pressure \\
\hline$P_{c}$ & : hydraulic pressure \\
\hline $\operatorname{Pr}$ & : Prandtl number, $\operatorname{Pr}=v / \alpha$ \\
\hline $\mathrm{q}_{\mathrm{i} \text { Conv }}$ & : convection heat transfer flux at the interior dome wall \\
\hline $\mathrm{q}_{\mathrm{cond}}$ & : heat transfer flux by pure conduction through the domed enclosure \\
\hline $\mathrm{q}_{\mathrm{o} \text { Conv }}$ & : convection heat transfer flux at the exterior dome wall \\
\hline $\mathrm{r}$ & : position radius \\
\hline$r^{*}$ & : dimensionless position radius $\left(\mathrm{r}^{*}=\mathrm{r}^{*} / \mathrm{L}_{\mathrm{max}}\right)$ \\
\hline $\mathrm{R}_{\mathrm{i}}$ & : interior dome radius \\
\hline $\mathrm{R} *_{\mathrm{i}}$ & : dimensionless radius of interior dome surface \\
\hline $\mathrm{R}_{\mathrm{o}}$ & : exterior dome radius \\
\hline $\mathrm{R} *_{\mathrm{o}}$ & : dimensionless radius of exterior dome surface \\
\hline $\mathrm{Ra}$ & : Rayleigh number $(\mathrm{Ra}=\mathrm{Gr} \cdot \mathrm{Pr})$ \\
\hline $\mathrm{Ra}^{*}$ & : modified Rayleigh number, equation (3.7) \\
\hline $\mathrm{T}$ & : temperature \\
\hline $\mathrm{T}_{\mathrm{i}}$ & : temperature of the inner dome wall \\
\hline $\mathrm{T}_{\mathrm{o}}$ & : temperature of the outer dome wall \\
\hline $\mathrm{T}^{*}$ & : dimensionless temperature $\mathrm{T}^{*}=\left(\mathrm{T}-\mathrm{T}_{\mathrm{o}}\right) /\left(\mathrm{T}_{\mathrm{i}}-\mathrm{T}_{\mathrm{o}}\right)$ \\
\hline $\mathrm{t}$ & : time \\
\hline $\mathrm{u}_{\mathrm{r}}$ & : velocity components in the $\mathrm{R}$ direction \\
\hline
\end{tabular}




\section{Greek Symbols}

$\beta$

$\varepsilon$

$\delta$

$\delta$

$\mu$

v

$\theta$

$\theta_{0}$

$\rho$

$\tau$

$\tau_{\mathrm{xy}}$
: velocity components in the $\theta$ direction

: dimensionless velocity components in the $\mathrm{r}$ direction

: dimensionless velocity components in the $\theta$ direction

: fluid velocity

\section{Description}

: fluid thermal expansion coefficient

: distance between the center of the interior and exterior surfaces (eccentricity)

: dimensionless gap spacing between dome layers $\left(\delta=L / R_{i}\right)$

: dimensionless gap spacing between dome layers $\left(\delta^{\prime}=\mathrm{L} / \mathrm{R}_{\mathrm{o}}\right)$

: dynamic viscosity

: fluid kinematic viscosity

: position angle

: dome truncation angle

: fluid density

: dimensionless time

: shear stress 


\section{CHAPTER 1}

\section{LITERATURE REVIEW}

\subsection{Introduction}

Domed skylights are perhaps the most important architectural design elements to deliver daylight and solar heat into buildings and connect the occupants to the outdoor environment. Using renewable energy sources such as daylight and solar heat is one of the requirements for sustainable building design, which aims to minimize building energy use and environmental impact, and improves the quality of the built environment for occupants. Domed skylights suit almost any type of building, with small size domes found in residential buildings, houses and retail stores, and large size domes found in industrial buildings and shopping malls. If properly designed, domed skylights may reduce building energy usage for lighting, cooling and heating. In addition, bringing daylight indoors and connecting occupants to the outdoors may have positive effects on occupant mood, health and well being. Despite their widespread use, the thermal performance of domed skylights has not been well understood. Current design tools, such as building energy simulation software and fenestration design computer tools do not cover such types of skylights. 
Domed skylights are made in different sizes, from low profiles to full hemispheres. The gap spacing between double glazed domed skylights may be uniform or non-uniform. The gap is typically filled with air.

\subsection{Overview}

This overview addresses convection heat transfer in domed skylights cavities with uniform/non-uniform cavity gap spacing. The first chapter presents basic background on natural convection heat transfer and heat transfer within and around skylight surfaces and the second chapter presents the mathematical modeling of natural convection in spherical coordinate systems and the boundary conditions of the problem at hand. In addition, Chapter 1 covers dimensionless governing equations and boundary conditions of natural convection within domed skylight cavities. The third chapter explains numerical modeling and numerical accuracy, as well as the validation of the numerical model. The fourth chapter presents numerical results, including streamlines, isotherms and Nusselt number for natural convection within domed skylight cavities with uniform/non-uniform gap spacing. The fifth chapter discusses the results and gives recommendation for future studies.

\subsection{Natural Convection Flow}

In natural convection, fluid motion is due to a fluid density gradient and body forces. The body force is gravitational, and the density gradient is due to a temperature 
gradient. The presence of a fluid density gradient in the gravitational field is the source of buoyancy-induced natural convection. Natural convection flow is a combination of fluid dynamics and heat transfer phenomena and coupling between these two phenomena make natural convection flow a complex subject to study. Often natural convection heat transfer equations are given dimensionless form for general application.

\subsection{Dimensionless Numbers}

The dimensionless variables involved in natural and forced convection include the Reynolds number (Re), Grashof number (Gr), Prandtl number (Pr), Rayleigh number (Ra) and Nusselt number $(\mathrm{Nu})$.

The Reynolds number is defined as the ratio of inertia to viscous forces in the velocity boundary layer. The Reynolds number is an important dimensionless variable in analyzing flow type when there is a substantial velocity gradient. It is defined as:

$$
\operatorname{Re}=\frac{V L}{v}
$$

where $V$ is the fluid velocity, $L$ is the characteristic length and $v$ is the kinematic viscosity $(\mu / \rho)$. The effect of Reynolds number is important in forced convection. 
The Grashof number characterizes the ratio of the buoyancy force to the viscous force acting on the fluid. The Grashof number in natural convection plays the same role as the Reynolds number in forced convection. It is defined as follows:

$$
G r=\frac{\beta g\left(T_{h}-T_{c}\right) L^{3}}{v^{2}}
$$

where $\beta$ is the volumetric thermal expansion coefficient, $g$ is the gravity acceleration, $T_{h}$ and $T_{c}$ are the hot and cold boundary temperatures, $\mathrm{L}$ is the characteristic length, and $v$ is the kinematic viscosity.

The Prandtl number is a dimensionless fluid property and is defined as the ratio of momentum diffusivity $(v)$ to thermal diffusivity $(\alpha)$ :

$$
\operatorname{Pr}=\frac{\mu C_{p}}{k_{f}}=\frac{v}{\alpha}
$$

where thermal diffusivity of the fluid is $\alpha=\mathrm{k}_{\mathrm{f}} / \rho \mathrm{C}_{\mathrm{p}}$

The Rayleigh number is the product of the Grashof number and the Prandtl number. It is defined as follows: 


$$
R a=G r \operatorname{Pr}=\frac{g \beta\left(T_{h}-T_{c}\right) L^{3}}{v \alpha}
$$

The Nusselt number characterizes the ratio of heat transfer by convection to the heat transfer by pure conduction and it is defined as follows:

$$
N u=\frac{q_{c o n v}}{q_{c o n d}}=\frac{h L}{k_{f}}
$$

where $q_{\text {cond }}=k_{f} A\left(T_{h}-T_{c}\right) / L$ and $q_{c o n v}=h A\left(T_{h}-T_{c}\right) . \mathrm{k}_{\mathrm{f}}$ is the fluid thermal conductivity at the mean temperature $T_{m}=\left(T_{h}+T_{c}\right) / 2, A$ is the surface area for heat transfer, and $\mathrm{h}$ is the convective heat transfer coefficient.

\subsection{Previous Work}

Heat transfer in double-glazed domed skylights is a complex subject to study. The heat transfer depends on the skylight's profile, gap thickness and boundary conditions. Previous studies are grouped into three different categories that are reviewed separately, as follows:

- Laminar natural convection within domed cavities

- Natural convection from the interior surface of domed skylights 
- Forced and natural convection from the exterior surface of domed skylights

\subsubsection{Natural Laminar Convection within Domed Cavities}

Although a few researchers have investigated heat transfer within concentric or vertically eccentric spheres and circular cylinders, there is very limited information available on heat transfer within domed cavities. Recently, Laouadi and Atif (2001) used the control volume method to address this and found the critical gap spacing that maximizes the heat transfer for different truncation angles in concentric domes when heated from outside under steady state conditions. They found that, for a small gap spacing ratios (gap spacing to radius ratio $\delta<0.1$ ), convective heat transfer in hemisphere domes (truncation angle of $90^{\circ}$ ) is about $13 \%$ higher than in low profile domes (truncation angle of $30^{\circ}$ ). They also developed the heat transfer correlations for uniform gap thickness in domed skylights as a function of dome profile, gap spacing, inner radius and Rayleigh number. The results apply when the interior surface is at a temperature lower than the exterior surface.

McGowan et al. (1998) used a numerical approach and found the Nusselt number for natural convection within pyramid and barrel vault (concentric half-circle cylinder) skylight cavities. McGowan et al. (1998) also used the thin boundary layer assumption to find the Nusselt number for natural convection from the interior surface of barrel vaults to the indoor environment. For natural laminar convection flow, they calculated the Nusselt number as follows: 


$$
N u_{\text {lam }}=\frac{2.8}{\ln \left(1+\frac{2.8}{N u_{\text {Thin }}}\right)}
$$

where $N u_{T h i n}=0.464 R a^{1 / 4}$

For natural convection turbulent flow McGowan et al. (1998) derived the Nusselt number as follows:

$$
N u_{\text {turb }}=0.122 R a^{1 / 3}
$$

McGowan et al. (1998) used the following equation to combine laminar and turbulent Nusselt numbers:

$$
N u=\left[\left(N u_{\text {lam }}\right)^{m}+\left(N u_{\text {turb }}\right)^{m}\right]^{1 / m}
$$

Bishop et al. (1964) set up experimental testing to study the flow patterns in concentric spheres for diameter ratios $\left(D_{\text {out }} / D_{\text {in }}\right)$ of $1.19,1.72$ and 3.14 when heated from inside. In their experiment, the temperature difference between the inner and outer spheres was $5^{\circ}, 15^{\circ}, 30^{\circ}$ and $60^{\circ} \mathrm{F}$. They visualized three different flow patterns, depending on the gap size and temperature differences. The first flow pattern, named the "crescent-eddy" type, occurred at a small temperature difference, at the largest diameter ratio (outer diameter to inner diameter ratio of $D_{o} / D_{i}=3.14$ ) and at the smallest 
diameter ratio $\left(D_{o} / D_{i}=1.19\right)$, while, for the intermediate diameter ratio $\left(D_{o} / D_{i}=1.72\right)$, it happens for all temperature differences $\left(5^{\circ} F<\Delta T<30^{\circ} F\right)$. They found a thin layer of high speed fluid near the spheres and slow moving fluid at the central crescent-eddy. The second flow pattern, named the "kidney-shaped-eddy" type, occurred at the large diameter ratio at a moderate to large temperature difference. The third flow pattern, named the "falling-vortices" type, occurred at a small diameter ratio and at moderate to high temperature differences. This is an unsteady flow characterized by the formation and shedding of vortex cells. These vortices appear in the upper region near the symmetric axis in counter-rotating pairs. Upon the formation of the second pair of counter-rotating cells, they merged together. Bishop et al. (1966) extended the previous experiment and established the convective heat transfer correlation in concentric spheres for diameter ratios from 1.19 to 3.14 when the Grashof number varied from $2 \times 10^{4}$ to $3.6 \times 10^{6}$.

Yin et al. (1973) studied the heat transfer within concentric spherical cavities with the diameter ratio $\left(\mathrm{D}_{0} / \mathrm{D}_{\mathrm{i}}\right)$ in the range of 1.09 to 2.17 for Grashof numbers in the range of $1.7 \times 10^{3}$ to $1.5 \times 10^{7}$. Yin et al. (1973) defined the transition Grashof number as the value of the Grashof number at which any unsteadiness can be observed in the flow field. They found that the transition Grashof number is dependent on the diameter ratio. They also found the transition Grashof number for different inverse relative gap thickness $\left(D_{i} / L\right)$. Their experiments showed that the transition Grashof number decreases as the inverse relative gap thickness increases. 
Caltagirone et al. (1980) studied heat transfer and flow patterns between concentric spheres numerically using a two-dimensional axisymmetric stream function. Their study was limited to small Rayleigh numbers from $3 \times 10^{3}$ to $5 \times 10^{4}$. They found mono-cellular flow, for relatively small Rayleigh numbers and for high Rayleigh numbers, depending on the initial conditions, and the solution might converge to mono-cellular or bi-cellular flow. Their solution converged to bi-cellular flow when a negative velocity is imposed at the top of the cavity.

Chiu and Chen (1996) investigated transient convection heat transfer between concentric and vertically eccentric spheres. They used a numerical model to study the case of heating from inside when the radius ratio is two, with air filling the cavity. They used three different setups; concentric, outer sphere's center below the inner sphere's center and outer sphere's center above the inner sphere's center to investigate the convective heat transfer. The numerical results showed that the heat transfer and flow patterns depend on the Rayleigh number and eccentricity. For concentric spheres and for vertically eccentric with the outer sphere's center above the inner sphere's center, they found only one steady vortex-cell flow for Rayleigh numbers up to $10^{5}$, but for the outer sphere's center below the inner sphere's center they found multi-cellular flow, which start to form at a critical Rayleigh number $R a=10^{5}$. At first, a vortex cell formed on top of the main cell, then as time passes, another cell appears in the bottom part of the cavity. They also found that heat transfer is greater if the inner sphere's center is lower than the outer sphere. 


\subsubsection{Natural Convection from Interior Surface of Dome}

Although there are some experimental and analytical studies available on natural convection for different geometries, such as long cylinders and spheres, there is very little information available on natural convection from concave surfaces. Chen and Cheng (2002) designed an experimental investigation for flow visualization to validate their numerical model of heat transfer and flow in an inclined arc-shape enclosure. They assumed that the curved surface is hotter than the flat surface. They found that the Nusselt number is appreciable for Grashof numbers larger than $10^{4}$, and with increasing Grashof number, the overall Nusselt number will increase. Analyzing the flow pattern for the inclination angles of $0^{\circ}$ and $180^{\circ}$, they found two symmetric cells when $10^{4}<\mathrm{Gr}$ $<10^{7}$, but when the inclination angle is $180^{\circ}$, there is thermal stratification and symmetric vortices are relatively weaker than the case when the inclination angle is $0^{\circ}$. For other angles, they visualized a one-cell pattern and a thermal boundary forming along the flat wall. From the numerical results, they suggested the correlation between Nusselt number and Grashof number as:

$$
N u=a(\theta) G r^{b(\theta)} \quad \text { for } \operatorname{Pr}=0.7 \text { and } 10^{4} \leq G r \leq 10^{7}
$$

where

$$
\begin{aligned}
& a(\theta)=\frac{1}{0.5155-0.0277 \theta^{2.3139}} \\
& b(\theta)=\frac{0.1539-0.0471 \theta}{1-0.2729 \theta-0.00681 \theta^{2}}
\end{aligned}
$$


Yovanovich (1987) and Chow and Yovanovich (1982) suggested $\sqrt{A}$ as a characteristic length to define the Nusselt number where $A$ is the surface area of heat transfer. When there is almost no motion $\left(N u_{C O N D}\right)$, heat transfers just by conduction. They performed different experiments and found $N u_{C O N D}$ for a wide range of geometries. They found that the quantity of $N u_{C O N D}$ is highly insensitive to the body shape and only varies from 3.391 to 3.609 over a very wide range of shapes, including cubes, lenses, and ellipsoids. Yovanovich (1987) defined the Nusselt number for natural convection as:

$$
N u_{\sqrt{A}}=N u_{C O N D}+c R a_{\sqrt{A}}^{(1 / 4)}
$$

where $\sqrt{A}$ is the characteristic length. The constants $c$ and $N u_{C O N D}$ can be found from the tables of Yovanovich et al. (1987).

Raithby and Hollands (1975) used a thin boundary layer approach to find a general correlation for the Nusselt number. They categorized flow over a surface in to two different types; attached flow or detached flow. Attached flow happens when the surface angle is $90^{\circ} \leq \zeta \leq 180^{\circ}$ and detached flow happens when $0^{\circ} \leq \zeta \leq 90^{\prime \prime}$.

Figure 1.1, shows the surface angle, attached-flow and detached-flow. The surface angle is the angle between $\hat{b}$ and $\hat{n}$, where $\hat{b}$ is the unit vector in the direction of the buoyancy force and $\hat{n}$ is the outward unit normal vector of the surface. Attached flow 
occurs in the case of a hot concave surface or a cold convex surface, and detached flow happens for the case of a cold concave surface or a hot convex surface.

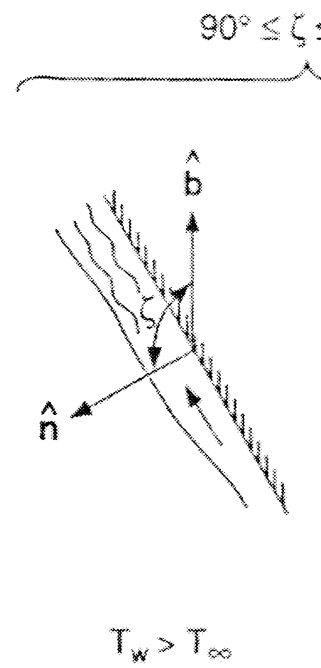

(a)

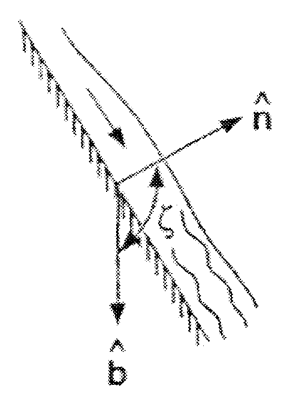

$T_{w}<T_{\infty}$

(b)

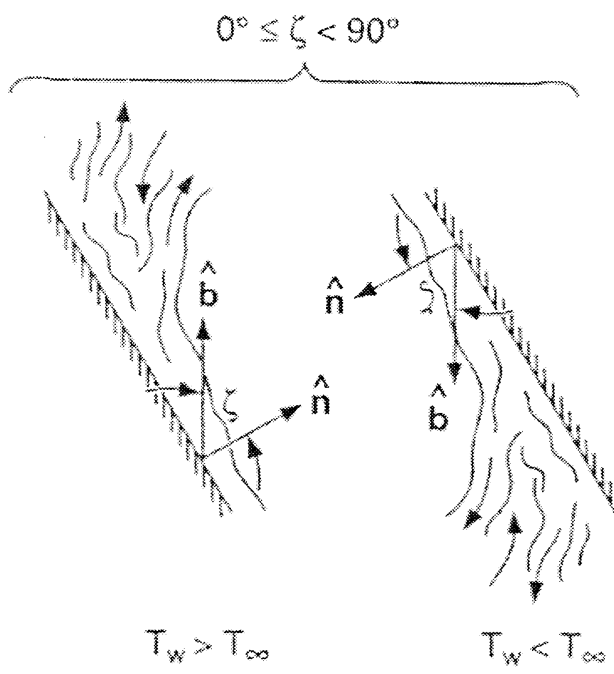

(c)

(d)

Figure 1.1: Definition of surface angle $\zeta$ for heated and cooled surface. For $90^{\circ} \leq \zeta \leq 180^{\circ}$ the flow remains attached, while it may detach if $0^{\circ} \leq \zeta \leq 90^{\circ}$ (Raithby. and Hollands, 1998)

Although their equation gives a rough estimate for natural convection, in reality there is no thin boundary layer for natural convection. Therefore, this correlation should be corrected to account for the thick boundary layer. Hassani and Hollands (1989) suggest the following correction for the thin boundary layer axisymmetric laminar natural convection:

$$
N u_{\text {lam }}=N u_{C O N D}+N u^{T h i n}
$$


And for long horizontal isothermal circular cylinders, they suggested the following correction to the thin boundary layer assumption:

$$
N u_{\text {lam }}=\frac{C_{1}}{\ln \left(1+\frac{C_{1}}{N u^{\text {Thin }}}\right)}
$$

where $\quad C_{1}=\frac{2 \pi L}{P_{i}}, \quad L=2 R, \quad P_{i}=$ Perimeter

These equations are valid for laminar flow. For the case of fully turbulent natural convection, they found that the local Nusselt number is independent of how far the point is from the leading edge and that it is only a function of the local surface angle $\zeta$ and Rayleigh number, and it can be integrated over the surface area to find the average Nusselt number. The average Nusselt number is:

$$
\overline{N u}_{t u r}=\bar{C}_{t}(\zeta) R a^{1 / 3}
$$

where $\bar{C}_{t}(\zeta)$ is defined as follows:

$$
\begin{aligned}
& \bar{C}_{t}(\zeta)=\frac{1}{A} \int_{A} C_{t}(\zeta) d A \\
& C_{t}(\zeta)=\left[C_{t}^{U}[\cos \zeta, 0]_{\max }, C_{t}^{V} \sin ^{1 / 3} \zeta\right]_{\max }, C_{t}^{U}=0.14\left(\frac{1+0.0107 \operatorname{Pr}}{1+0.01 \operatorname{Pr}}\right), C_{t}^{V}=\left(\frac{0.13 \operatorname{Pr}^{0.22}}{\left(1+0.61 \operatorname{Pr}^{0.81}\right)^{0.42}}\right)
\end{aligned}
$$


When there is a mixture of natural laminar and turbulent natural convection, the Nusselt number can be found from blending the laminar and turbulent Nusselt number using the following equation:

$$
N u=\left[\left(N u_{\text {lam }}\right)^{m}+\left(N u_{\text {tur }}\right)^{m}\right]^{1 / m}
$$

The value of ' $m$ ' depends on shape and is found from curve-fitting to experimental results. Fore some shapes, the value of ' $m$ ' can be found from Raithby and Hollands (1998).

\subsubsection{Forced and Natural Convection from Exterior Surface of Dome}

In the outdoor environment, usually the wind element is an important factor and has significant effect on heat transfer. Thus, heat transfer around the exterior surface of domed skylight is usually by forced convection. In the absence of wind, heat transfer occurs by natural convection. Previous studies on forced and natural convection will be

reviewed separately. For natural convection, same as the natural convection from interior surface of dome, two different cases of natural laminar and turbulent convection should be considered. Studies of forced convection heat transfer from a curved surface to its adjacent environment were very scarce, covering only flows over simple shapes such as spheres, cylinders, cubes, etc. 
Ziskind et al. (2001) setup an experimental investigation on heat transfer from a heated small sphere in a horizontal flow. The flow velocity was in the range of $0-0.1 \mathrm{~m} / \mathrm{s}$ "low Reynolds number". Ziskind et al. (2001) considered free convection, forced convection, and a combination of both. In the case of mixed convection, it is common to study three basic conditions: (1) "assisting" regime, where the direction of the forced flow coincides with that of the free flow; (2) "opposing" regime, where the direction of the forced flow is opposite to that of the free flow; and (3) "crossing" regime, where the direction of the forced flow is normal to that of the free flow. Any other setting can be deduced based on the results for the above-mentioned three scenarios. It is evident that, in the mixed cross flow regime, as the velocity decreases, the free convection dominates, and that, as the velocity increases, the forced convection dominates the free convection. Their results match well with the following equations for pure forced and free convection respectively suggested by Bird et al. (1960):

$$
\begin{aligned}
& N u=2+0.6 \operatorname{Re}^{1 / 2} \operatorname{Pr}^{1 / 3} \quad \text { (Forced convection) } \\
& N u=2+0.6 \mathrm{Gr}^{1 / 4} \operatorname{Pr}^{1 / 3} \quad(\text { Free convection })
\end{aligned}
$$

They used the ratio of the Grashof number to the square of the Reynolds number, $\mathrm{Gr} / \mathrm{Re}^{2}$, in order to compare the effects of forced and free convection. They found that free convection is negligible when $\mathrm{Gr} / \mathrm{Re}^{2} \leq 0.02-0.03$. 
Melissari and Argyropoulos (2005) used a mathematical model of the melting sphere and solved it numerically to find the correlation of heat transfer in forced convection over a sphere. They suggested the following correlation to find the Nusselt number for a wide range of Prandtl numbers $(0.003<\operatorname{Pr}<10)$ :

$$
\begin{aligned}
& N u=2+0.47 \operatorname{Re}^{1 / 2} \operatorname{Pr}^{0.36} \\
& \text { for } \\
& 3 \times 10^{-3}<\operatorname{Pr}<10 \text { and } 10^{2}<\operatorname{Re}<2 \times 10^{4}
\end{aligned}
$$

Previously, Melissari and Argyroulos (2004) had concluded that, for values of the buoyancy parameter lower than the range of $G r / \operatorname{Re}^{2}=0.5-1$, the total melting time is not affected by natural convection. They compared their computational results with different experimental results with a wide range of Prandtl numbers (liquid metal to water). The comparison showed good agreement between predictions from the derived correlation and experimental results.

Pop and Yan (1998) performed an analytical study on forced convection over a circular cylinder and a sphere for large Peclet numbers. They assumed steady state heat transfer from the circular cylinder and sphere, which they maintained at constant temperature. Most of the researchers report the average heat transfer over a circular cylinder and sphere. Pop and Yan, however, used an analytical approach to find the local and average Nusselt number for both the sphere and the cylinder. Their equations match very well with the experimental and numerical results by other researchers. They studied 
heat transfer around the circular cylinder for steady state forced convection when both cylinder and ambient temperature are constant, and the ambient temperature is lower than cylinder's temperature. Their results are summarized as:

$$
\begin{aligned}
& \frac{N u_{\theta}}{P e^{1 / 2}}=\frac{2}{\sqrt{\pi}} \sin \left(\frac{\theta}{2}\right) \\
& \frac{\overline{N u}}{P e^{1 / 2}}=0.71825
\end{aligned}
$$

where the Peclet number is defined as $P e=\operatorname{Re} \operatorname{Pr}$ and $\theta=0^{\circ}$ is the horizontal flow direction. They followed the same steps and found local and average Nusselt numbers for steady state forced convection heat transfer from a hot isothermal sphere. The ambient temperature is assumed constant. Their results are summarized as:

$$
\begin{aligned}
& \frac{N u_{\theta}}{P e^{1 / 2}}=\frac{3}{\sqrt{2 \pi}} \frac{1-\eta}{(2-\eta)^{1 / 2}} \text { where } \eta=\cos \theta \\
& \frac{\overline{N u}}{P e^{1 / 2}}=0.79788
\end{aligned}
$$

Alassar (2005) used the stream function approach to solve the time-dependent Navier-Stokes and energy equations for small Reynolds numbers in flow over spheroid bodies to find the average Nusselt number. He investigated the problem for hot spheroid bodies for axis ratios between 0.55 and 0.99 at three Reynolds numbers: 10, 40 and 100 . 
To study the effect of Reynolds number, he considered a wide range of Reynolds numbers (from 10 to 500 ) for the axis ratio of 0.75 . He observed about a $12 \%$ increase in the average Nusselt number by changing the geometry from a sphere to a spheroid with an axis ratio of 0.55 at all Reynolds numbers considered.

Hilpert (2002) suggested the following empirical equation to find the average Nusselt number over a cylinder with different cross sections.

$$
N u_{D}=C \operatorname{Re}_{D}^{m} \operatorname{Pr}^{1 / 3}
$$

where $\operatorname{Re}_{D}$ is Reynolds number based on a characteristic length, $\mathrm{D}$, which can be calculated from the following equation:

$$
\begin{gathered}
\operatorname{Re}_{D}=\frac{\rho D U_{\infty}}{\mu} \\
D=\frac{\text { Perimiter }}{\pi}
\end{gathered}
$$

The characteristic length $D$ is the equivalent circular diameter and the values of the constants $C$ and $m$ are found from curve fitting to the experimental data. The values of $C$ and $m$ for different shapes can be found from Jacob (1949). 


\subsection{Conclusion}

Natural convection heat transfer for spheres and cylinders was studied experimentally and numerically. Laouadi and Atif (2001) derived the correlation of the Nusselt number for natural convection within domed skylight cavities with uniform gap spacing when heated from outside. To the knowledge of the author, there is no study in domed skylights with uniform gap spacing when heated from inside and domed skylights with nonuniform gap spacing. Therefore, this research will study laminar natural convection heat transfer within domed skylights with uniform gap spacing when heated from inside and domed skylights with non-uniform gap spacing when heated from outside.

\subsection{Scope of Research}

This work is a part of the research collaboration between National Research Council of Canada (NRC) and Ryerson University to improve the capabilities of NRC software regarding prediction of thermal performance of skylights. The study addresses convection heat transfer in the domed cavities. This information is needed to compute the thermal performance (e.g., U-value) of domed skylights. The specific objectives are:

- To investigate the flow pattern and thermal field in domed cavities with uniform/non-uniform gaps.

- To develop correlations for Nusselt number as a function of the dome shape parameters and Grashof number. 
The results of this study will be implemented into NRC software: SkyVision (2007) and Daylight 1-2-3 (2007). SkyVision is a freeware specialized computer program to calculate the overall optical, thermal and daylighting performance of fenestration systems (windows, shadings, skylights). Daylight 1-2-3 is freeware nonexpert daylighting/energy analysis software to support design professionals and architectural students in dealing with daylighting-related design decisions in side-lit or top-lit commercial buildings during the initial design and design development stages. Daylight 1-2-3 integrates three computer programs - Radiance (for illuminance calculations), SkyVision (for optical and thermal calculation of fenestration systems) and ESP-r (for whole building energy calculation) - to calculate building requirements for lighting, heating and cooling energy.

\subsection{Approach}

This study will use numerical modelling and CFD computer simulation software to find the Nusselt number in domed skylights for different types of geometry. For each type of geometry, a power function will curve-fit for the Nusselt number. Subsequently, another equation, which is a function of domed skylight gap spacing, will curve-fit for each coefficient of the power functions to develop a correlation for the Nusselt number as a function of Rayleigh number and gap spacing. 


\section{CHAPTER 2}

\section{MATHEMATICAL FORMULATION}

\subsection{Introduction}

In order to determine the effect of domed skylights on cooling and heating loads of the building, the heat transfer and fluid flow in domed skylight cavities have been studied parametrically for a wide range of geometries and thermo-physical conditions. The general governing equations of continuity, Navier-Stokes and energy were simplified for the geometry of the problem at hand.

\subsection{Modeling The Geometry}

A double glazed domed skylight with uniform and non-uniform gap spacing is the subject of interest. Figure 2.1 shows a section of a spherical cap with non-uniform gap spacing. The $\mathrm{Y}$-axis is the rotation axis. The dome cavity is characterized by its interior and exterior radii $\left(\mathrm{R}_{\mathrm{i}}, \mathrm{R}_{\mathrm{o}}\right)$, minimum and maximum gap thicknesses $\left(\mathrm{L}_{\min }, \mathrm{L}_{\max }\right)$, truncation angle $\left(\theta_{0}\right)$ and eccentricity $(\varepsilon)$. The center of the exterior surface is on the origin of the coordinate system and the center of the interior surface (A) is at distance of $\varepsilon$ on the $\mathrm{Y}$-axis below the origin. The inner radius is always greater than or equal to the outer radius and $\varepsilon$ may take negative or zero values. The edge of the dome (BC) is along the outer radius $\left(R_{0}\right)$. The truncation angle $\left(\theta_{0}\right)$ is the angle between the Y-axis and OC. 
The maximum gap spacing $\left(\mathrm{L}_{\max }\right)$ is measured at the top of the cavity and the minimum gap spacing $\left(\mathrm{L}_{\min }\right)$ is measured at the edge of dome.

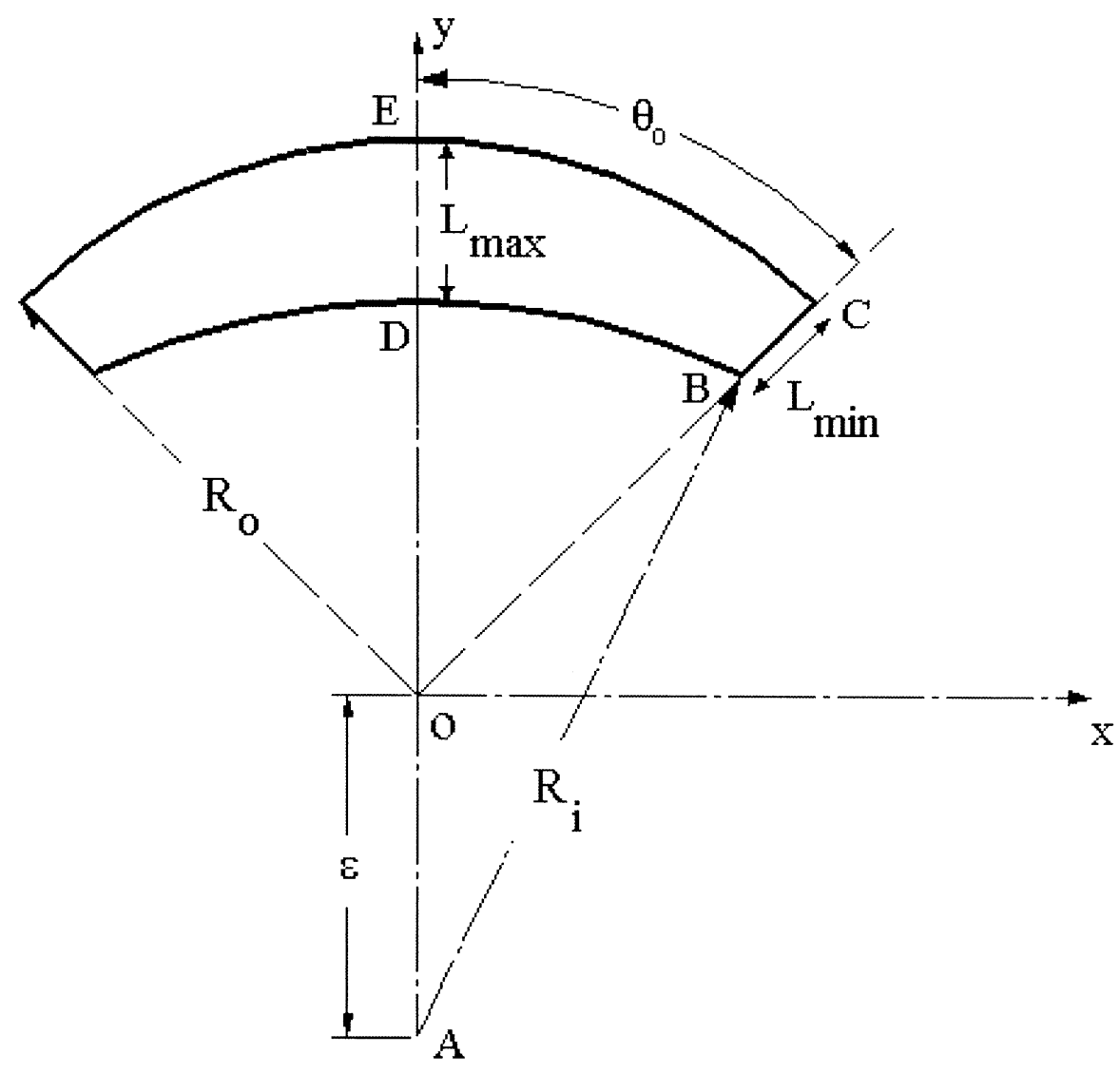

Figure 2.1: Section view of a spherical cap

Different geometries may be generated by varying the truncation angle, inner radius, outer radius, eccentricity, minimum gap thickness and maximum gap thickness. However, these variables are not all independent. The maximum gap thickness is assumed to be constant and assigned a unit length. Each geometry is defined by the truncation angle, the inner radius or the outer radius and the ratio of maximum to 
minimum gap thicknesses. Knowing the outer radius, the inner radius can be determind from the other parameters. From Figure 2.1 it can be concluded that:

$$
\begin{aligned}
& O D=O E-D E=R_{o}-L_{\text {max }} \\
& O D=A D-D O=R_{i}-\varepsilon
\end{aligned}
$$

Finding $\varepsilon$ from these two equations:

$$
\mathcal{E}=R_{i}-\left(R_{o}-L_{\max }\right)
$$

In triangle $\mathrm{AOB}$, from the vector algebra, the distance ' $\mathrm{AB}$ ' is the sum of distances 'AO' and 'OB':

$$
A O B: R_{i}^{2}=\left(R_{o}-L_{\min }\right)^{2}+\varepsilon^{2}-2 \varepsilon\left(R_{o}-L_{\min }\right) \cos \left(\pi-\theta_{0}\right)
$$

Defining $a$ and $b$ as follows:

$$
R_{o}-L_{\min }=a, \quad R_{o}-L_{\max }=b
$$

then, substituting Equation 2.2 and 2.4 into Equation 2.3 gives:

$$
R_{i}^{2}=a^{2}+\left(R_{i}-b\right)^{2}+2\left(R_{i}-b\right) a \cos \theta_{0}
$$


After simplification of Equation 2.5, one finds $R_{i}$ :

$$
R_{i}=\frac{a^{2}+b^{2}-2 a b \cos \theta_{0}}{2\left(b-a \cos \theta_{0}\right)}
$$

Substituting the original value of $a$ and $b$ into Equation 2.6, one obtains:

$$
R_{i}=\frac{\left(R_{o}-L_{\min }\right)^{2}+\left(R_{o}-L_{\max }\right)^{2}-2\left(R_{o}-L_{\min }\right)\left(R_{o}-L_{\max }\right) \cos \theta_{0}}{2\left(\left(R_{o}-L_{\max }\right)-\left(R_{o}-L_{\min }\right) \cos \theta_{0}\right)}
$$

It is useful to use the dimensionless ratio $R_{i} / R_{o}$ in Equation 2.7 to get a general solution. To this end, the dimensionless gap thicknesses may be defined as follows:

$$
\frac{L_{\max }}{R_{o}}=\delta_{\text {max }}^{\prime} \quad, \quad \frac{L_{\min }}{R_{o}}=\delta_{\text {min }}^{\prime}
$$

Dividing Equation 2.7 by $R_{o}$ and substituting Equation 2.8 into it gives the following relation:

$$
\frac{R_{i}}{R_{o}}=\frac{\left(1-\delta_{\text {min }}^{\prime}\right)^{2}+\left(1-\delta_{\text {max }}^{\prime}\right)^{2}-2\left(1-\delta_{\text {min }}^{\prime}\right)\left(1-\delta_{\text {max }}^{\prime}\right) \cos \theta_{0}}{2\left(\left(1-\delta_{\text {max }}^{\prime}\right)-\left(1-\delta_{\text {min }}^{\prime}\right) \cos \theta_{0}\right)}=d
$$


Note that if $\varepsilon=0$ then, $L_{\text {max }}=L_{\text {min }}=L$ and $\delta_{\text {max }}^{\prime}=\delta_{\text {min }}^{\prime}=\delta^{\prime}$. In this case, Equation 2.9 will simplify to:

$$
\frac{R_{i}}{R_{o}}=1-\delta^{\prime}
$$

\subsection{Assumptions}

The general governing equations should be simplified for the geometry of the problem and physical properties. Typical assumptions that are made to simplify the problem at hand are: Newtonian fluid, constant physical properties, Boussinesq approximation, incompressible flow, axisymmetric flow or two-dimensional flow. Each assumption will be discussed in more detail in this chapter. There are also some ideal conditions assumed for the boundary conditions.

\subsubsection{Newtonian Fluid}

The fluid inside the cavity is assumed to be Newtonian, where the stress tensor, and hence the shear-stress tensor, is linearly related to the deformation-rate tensor. Almost all gases are Newtonian fluids. The following Equation 2.11 defines a Newtonian fluid.

$$
\tau_{x y}=\tau_{y x}=\mu\left(\frac{\partial u_{x}}{\partial y}+\frac{\partial u_{y}}{\partial x}\right)
$$


where $\mu$ is the dynamic viscosity of fluid.

\subsubsection{Physical Properties and Boundary Conditions}

All physical properties of the fluid are assumed constant (i.e., $d C_{p} / d T=d \mu / d T=$ $d k / d T=0)$ in the range of the temperature differences under consideration. Due to the small temperature differences that are encountered in skylight applications, the assumption of constant thermal properties is a good approximation. The edges of the dome skylight are assumed adiabatic. The latter assumption is based on the assumption that heat transfer from the edges can be neglected with respect to the heat transfer from the dome surfaces. The temperature of the outer and inner surface of the cavity is assumed uniform and constant.

\subsubsection{Boussinesq Approximation and Incompressible Flow}

The fluid is assumed to be incompressible, which holds well for gases at low velocity. The incompressible flow assumption results in the density being constant. But the changes in density due to thermal expansion are the driving force in natural convection and cannot be neglected. To solve this issue, the Boussinesq assumption considers changes in density only in the gravitational body-force term of the momentum equations. The changes in density in other terms are assumed to be small and are neglected. The temperature difference is assumed to be small enough to have a negligible effect on fluid properties. Therefore, constant fluid properties at a mean fluid temperature are considered constant for the problem. 
The Boussinesq approximation is valid when the temperature difference between hot and cold surfaces is very small. This criteria is defined as:

$$
\beta\left(T_{h}-T_{c}\right)<<1
$$

where $T_{h}$ and $T_{c}$ are hot and cold surface temperatures respectively and $\beta$ is the volumetric thermal expansion coefficient, defined as follows:

$$
\beta=-\frac{1}{\rho_{r e f}}\left(\frac{\partial \rho}{\partial T}\right)_{P=c o n s t}
$$

With the Boussinesq approximation, density changes linearly with temperature.

Detailed steps on the simplification of the momentum equations using the Boussinesq approximation can be found in Oosthuizen and Naylor (1999). The Boussinesq approximation can be expressed as:

$$
\rho-\rho_{\text {ref }} \approx-\rho_{\text {ref }} \cdot \beta\left(T-T_{r e f}\right)
$$

Gray and Giorgini (1975) found that the Boussinesq approximation is valid for a Newtonian fluid when $\left(T_{h}-T_{c}\right) \leq 28.6^{\circ} \mathrm{C}$. 


\subsubsection{Axisymmetric Two-Dimensional Flow}

The flow in domed skylight cavities can be classified into two-dimensional or axisymmetric flow. The flow is axisymmetric when the flow is laminar and takes place near the bodies that are generated by revolving a two-dimensional shape around a vertical (parallel to gravity) line. The flow is two dimensional when the flow is along a body that has an invariant cross section along the horizontal axis.

\subsection{Governing Equations}

The governing equations are cast in the spherical coordinate system. Figure 2.2 shows a section of a spherical cap with a truncation angle of $\theta_{\mathrm{o}}$ and maximum gap thickness $\mathrm{L}_{\max }$.

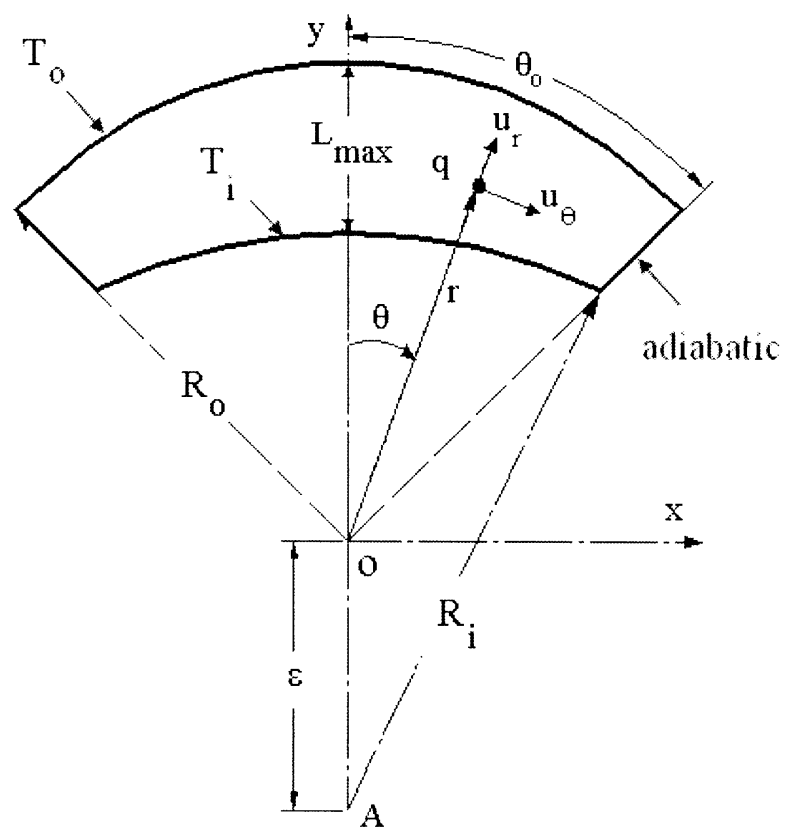

Figure 2.2: Spherical coordinates system for a domed cavity. 


\subsubsection{Fluid Flow in Domed Cavities}

Using the foregoing assumptions, the governing equations for continuity, momentum, and energy are cast as follows (Bird et al. 2001):

Continuity:

$$
\frac{1}{r} \frac{\partial}{\partial r}\left(r^{2} u_{r}\right)+\frac{1}{\sin \theta} \frac{\partial}{\partial \theta}\left(u_{\theta} \sin \theta\right)=0
$$

r-momentum:

$$
\begin{array}{r}
\rho\left[\frac{\partial u_{r}}{\partial t}+u_{r} \frac{\partial u_{r}}{\partial r}+\frac{u_{\theta}}{r} \frac{\partial u_{r}}{\partial \theta}-\left.\frac{u_{\theta}^{2}}{r}\right|_{\rfloor}=-\frac{\partial P}{\partial r}-\rho g_{r}+\right. \\
\mu\left[\frac{1}{r^{2}} \frac{\partial^{2}}{\partial r^{2}}\left(r^{2} u_{r}\right)+\frac{1}{r^{2} \sin \theta} \frac{\partial}{\partial \theta}\left(\sin \theta \frac{\partial u_{r}}{\partial \theta}\right)_{\rfloor}\right.
\end{array}
$$

$\theta$-momentum:

$$
\begin{aligned}
& \rho\left[\frac{\partial u_{\theta}}{\partial t}+u_{r} \frac{\partial u_{\theta}}{\partial r}+\frac{u_{\theta}}{r} \frac{\partial u_{\theta}}{\partial \theta}+\left.\frac{u_{r} u_{\theta}}{r}\right|_{]}=-\frac{1}{r} \frac{\partial P}{\partial \theta}-\rho g_{\theta}+\right. \\
& \mu\left[\frac{1}{r^{2}} \frac{\partial}{\partial r}\left(r^{2} \frac{\partial u_{\theta}}{\partial r}\right)+\frac{1}{r^{2}} \frac{\partial}{\partial \theta}\left(\frac{1}{\sin \theta} \frac{\partial}{\partial \theta}\left(u_{\theta} \sin \theta\right)\right)+\frac{2}{r^{2}} \frac{\partial u_{r}}{\partial \theta}\right\rceil
\end{aligned}
$$

energy:

$$
\begin{aligned}
\rho C_{p}\left[\frac{\partial T}{\partial t}+u_{r} \frac{\partial T}{\partial r}+\frac{u_{\theta}}{r} \frac{\partial T}{\partial \theta} \mid\right\rfloor= & k\left[\frac{1}{r^{2}} \frac{\partial}{\partial r}\left(r^{2} \frac{\partial T}{\partial r}\right)+\right. \\
& \left.\frac{1}{r^{2} \sin \theta} \frac{\partial}{\partial \theta}\left(\sin \theta \frac{\partial T}{\partial \theta}\right)\right\rfloor
\end{aligned}
$$


To apply the Boussinesq approximation in the previous equations, we define a pressure defect $p^{\prime}$ as a difference between the local pressure $(p)$ resulting from fluid motion and the hydrostatic pressure $\left(p_{c}\right)$ at the same level at the mean enclosure temperature $\left(T_{c}\right)$.

$$
p^{\prime}=p-p_{c}=p-\rho_{c} g r \cos \theta
$$

Finding pressure $(p)$ from Equation 2.19 and then calculating pressure gradient in the $r$ direction, one obtains:

$$
\frac{\partial p}{\partial r}=\frac{\partial p^{\prime}}{\partial r}-\frac{\partial\left(\rho_{c} g r \sin \theta\right)}{\partial r}=\frac{\partial p^{\prime}}{\partial r}-\rho_{c} g_{r}
$$

where $g_{r}=g \cos \theta$. Substituting Equation 2.14 and 2.20 into the r-momentum Equation (2.16) and simplifying the result, one gets:

$$
\begin{gathered}
\rho\left[\frac{\partial u_{r}}{\partial t}+u_{r} \frac{\partial u_{r}}{\partial r}+\frac{u_{\theta}}{r} \frac{\partial u_{r}}{\partial \theta}-\left.\frac{u_{\theta}^{2}}{r}\right|_{\rfloor}=-\frac{\partial P}{\partial r}+\rho \beta g_{r}\left(T-T_{i}\right)+\right. \\
\mu\left[\frac{1}{r^{2}} \frac{\partial^{2}}{\partial r^{2}}\left(r^{2} u_{r}\right)+\frac{1}{r^{2} \sin \theta} \frac{\partial}{\partial \theta}\left(\sin \theta \frac{\partial u_{r}}{\partial \theta}\right)_{\rfloor}^{]}\right.
\end{gathered}
$$

Following the same steps as above for the $\theta$-momentum equation reads as follows: 


$$
\begin{gathered}
\rho\left[\frac{\partial u_{\theta}}{\partial t}+u_{r} \frac{\partial u_{\theta}}{\partial r}+\frac{u_{\theta}}{r} \frac{\partial u_{\theta}}{\partial \theta}+\left.\frac{u_{r} u_{\theta}}{r}\right|_{\rfloor}=-\frac{1}{r} \frac{\partial P}{\partial \theta}+\rho \beta g_{\theta}\left(T-T_{i}\right)+\right. \\
\mu\left[\frac{1}{r^{2}} \frac{\partial}{\partial r}\left(r^{2} \frac{\partial u_{\theta}}{\partial r}\right)+\frac{1}{r^{2}} \frac{\partial}{\partial \theta}\left(\frac{1}{\sin \theta} \frac{\partial}{\partial \theta}\left(u_{\theta} \sin \theta\right)\right)+\left.\frac{2}{r^{2}} \frac{\partial u_{r}}{\partial \theta}\right|^{\prime}\right]
\end{gathered}
$$

\subsubsection{Boundary Conditions}

The foregoing Equations (2.15), (2.18), (2.21) and (2.22) are subject to the following boundary conditions:

- The inner and outer surfaces of the cavity are assumed isothermal and constant

- The edges of the cavity are assumed sealed and adiabatic.

- The flow is axis-symmetric at the $\mathrm{Y}$ axis. In this regard, the fluid velocity normal to the line of symmetry is zero.

- Non slip condition at the interior and exterior surfaces as well as all the edges.

In reality, the temperature varies along the surface, especially near the edges of the domed skylight (frame effect), but it is almost constant at the center. Curcija and Goss (1993) analyzed the effect of the edges on fenestration temperature surfaces and found that the temperature varies in a band of $63.5 \mathrm{~mm}$ around the fenestration edges and it is almost constant at the center. In the problem at hand, this band around the frame is very small compared to the size of the dome surface; and as a result, its effect can be neglected. Therefore, the isothermal assumption is a reasonable engineering 
approximation. In addition, the no-slip boundary condition is assumed at the boundary surfaces and at the edge of the cavity.

These boundary conditions are translated into the following mathematical conditions:

a. Edges: adiabatic and no-slip conditions

at $\theta=\theta_{0},\left(R_{o}-L_{\min }\right)<r<R_{o}: u_{\theta}=u_{r}=0, \frac{\partial T}{\partial \theta}=0$

b. Interior surface: isotherm and no-slip conditions ( $L$ is the local gap thickness at $\theta$ ) at $r=R_{o}-L, 0<\theta<\theta_{o}: u_{\theta}=u_{r}=0, \quad T=T_{i}$

c. Exterior surface: Isotherm and no-slip conditions at $r=R_{o}, 0<\theta<\theta_{0}: u_{\theta}=u_{r}=0, \quad T=T_{o}$

d. Symmetric line:

at $\theta=0$ for $R_{i}-\varepsilon<r<R_{o}: u_{\theta}=0, \frac{\partial u_{r}}{\partial \theta}=\frac{\partial T}{\partial \theta}=0$ 


\subsection{Dimensionless Governing Equations}

To obtain a general solution of the problem for any similar geometry, the governing equations are non-dimensionalized using the following dimensionless variables:

$$
\begin{aligned}
& r^{*}=\frac{r}{L_{\max }}, \tau=\frac{t}{\left(\frac{L_{\max }^{2}}{v}\right)}, \quad U_{r}=\frac{u_{r}}{\left(\frac{v}{L_{\max }}\right)}, \quad U_{\theta}=\frac{u_{\theta}}{\left(\frac{v}{L_{\max }}\right)}, \\
& P^{*}=\frac{p^{\prime}}{\rho_{i}\left(\frac{v}{L_{\max }}\right)^{2}}, \quad T^{*}=\frac{T-T_{i}}{T_{o}-T_{i}}
\end{aligned}
$$

Substituting the dimensionless variables, Equation (2.27), into the governing Equations (2.15), (2.18), (2.21) and (2.22), the dimensionless governing equations read as follows:

continuity:

$$
\frac{1}{r^{*}} \frac{\partial}{\partial r^{*}}\left(r^{* 2} U_{r}\right)+\frac{1}{\sin \theta} \frac{\partial}{\partial \theta}\left(U_{\theta} \sin \theta\right)=0
$$

r-momentum:

$$
\begin{gathered}
{\left[\frac{\partial U_{r}}{\partial \tau}+U_{r} \frac{\partial U_{r}}{\partial r^{*}}+\frac{U_{\theta}}{r^{*}} \frac{\partial U_{r}}{\partial \theta}-\left.\frac{U_{\theta}^{2}}{r^{*}}\right|_{\rfloor}=-\frac{\partial P^{*}}{\partial r^{*}}+G r T^{*} \cos \theta+\right.} \\
{\left[\frac{1}{r^{* 2}} \frac{\partial^{2}}{\partial r^{* 2}}\left(r^{* 2} U_{r}\right)+\frac{1}{r^{* 2} \sin \theta} \frac{\partial}{\partial \theta}\left(\sin \theta \frac{\partial U_{r}}{\partial \theta}\right)_{]}\right.}
\end{gathered}
$$


$\theta$-momentum:

$$
\begin{aligned}
& {\left[\frac{\partial U_{\theta}}{\partial \tau}+U_{r} \frac{\partial U_{\theta}}{\partial r^{*}}+\frac{U_{\theta}}{r^{*}} \frac{\partial U_{\theta}}{\partial \theta}+\left.\frac{U_{r} U_{\theta}}{r^{*}}\right|_{\rfloor}=-\frac{1}{r^{*}} \frac{\partial P^{*}}{\partial \theta^{*}}+G r T^{*} \sin \theta+\right.} \\
& {\left[\frac{1}{r^{* 2}} \frac{\partial}{\partial r^{*}}\left(r^{* 2} \frac{\partial U_{\theta}}{\partial r^{*}}\right)+\frac{1}{r^{* 2}} \frac{\partial}{\partial \theta}\left(\frac{1}{\sin \theta} \frac{\partial}{\partial \theta}\left(U_{\theta} \sin \theta\right)\right)+\left.\frac{2}{r^{* 2}} \frac{\partial U_{r}}{\partial \theta}\right|_{]}\right.}
\end{aligned}
$$

energy:

$$
\operatorname{Pr}\left[\frac{\partial T^{*}}{\partial \tau}+U_{r} \frac{\partial T^{*}}{\partial r^{*}}+\left.\frac{U_{\theta}}{r^{*}} \frac{\partial T^{*}}{\partial \theta}\right|_{\rfloor}=\frac{1}{r^{* 2}} \frac{\partial}{\partial r^{*}}\left(r^{* 2} \frac{\partial T^{*}}{\partial r^{*}}\right)+\frac{1}{r^{* 2} \sin \theta} \frac{\partial}{\partial \theta}\left(\sin \theta \frac{\partial T^{*}}{\partial \theta}\right)\right.
$$

where

$$
G r=\frac{\rho^{2} g \beta L_{\max }^{3}\left(T_{o}-T_{i}\right)}{\mu^{2}}, \operatorname{Pr}=\frac{\mu C_{p}}{k}
$$

Defining $L_{\min } / R_{o}=\delta_{\text {min }}^{\prime}, L_{\max } / R_{o}=\delta^{\prime}{ }_{\max }$ and $L / R_{o}=\delta^{\prime}$, where " $L$ " is the local gap thickness at an angle $\theta$, the dimensionless form of the boundary conditions are:

a. Edges: adiabatic and no-slip condition

$$
U_{\theta}=U_{r}=0, \frac{\partial T^{*}}{\partial \theta}=0 \text { at } \theta=\theta_{0}, \frac{1}{\delta_{\max }^{\prime}}-\frac{\delta_{\min }^{\prime}}{\delta_{\max }^{\prime}}<r^{*}<\frac{1}{\delta_{\max }^{\prime}}
$$

b. Interior surface: isotherm and no-slip conditions

$$
U_{\theta}=U_{r}=0, \quad T^{*}=0 \text { at } r^{*}=\frac{1}{\delta_{\max }^{\prime}}-\frac{\delta^{\prime}}{\delta_{\max }^{\prime}}, 0<\theta<\theta_{0}
$$


c. Exterior surface: isotherm and no-slip conditions

$$
U_{\theta}=U_{r}=0, \quad T=1 \text { at } r^{*}=\frac{1}{\delta^{\prime}{ }_{\max }}, 0<\theta<\theta_{0}
$$

d. Symmetric line,

$$
U_{\theta}=0, \quad \frac{\partial U_{r}}{\partial \theta}=\frac{\partial T^{*}}{\partial \theta}=0 \text { at } \theta=0, \frac{1}{\delta_{\text {max }}^{\prime}}-1<r^{*}<\frac{1}{\delta^{\prime}{ }_{\max }}
$$

\subsection{Evaluation of Heat Transfer}

Laminar natural convection within domed cavities is characterized by the Nusselt number. Nusselt number is defined as the ratio of the convective heat transfers to conductive heat transfer. The Nusselt number for the inner surface and outer surface are defined as follows:

$$
N u_{i}=\frac{q_{i_{\text {Conv }}}}{q_{\text {Cond }}}, \quad N u_{o}=\frac{q_{o_{\text {Conv }}}}{q_{\text {Cond }}}
$$

where $\mathrm{q}_{\mathrm{i} C o n v}$ and $\mathrm{q}_{\mathrm{oConv}}$ are the convection heat transfer from the inner surface and outer surface of domed cavity.

When the buoyancy force is very weak, heat transfers only by conduction. As a result, The Nusselt number in Equation 2.37 becomes unity. Under steady state 
conditions, the Nusselt numbers of the inner surface and outer surface are equal. In order to find the Nusselt number, conduction heat transfer and convection heat transfer are evaluated in the geometry of the problem in hand. Two types of geometry considered in this study including uniform gap thickness and non-uniform gap thickness. In this study the Nusselt number was calculated from the convection from the outer surface ( $\left.\mathrm{q}_{\mathrm{oConv}}\right)$. The convection heat transfer from the outer surface in Equation 2.37 is found from numerical procedures and the conduction heat transfer for uniform and non-uniform gap thicknesses were calculated in this section.

\subsubsection{Cavities with Uniform Gap Thickness}

Conduction heat transfer within a domed cavity in a spherical coordinate system is one dimensional and is defined as:

$$
q_{\text {Cond }}=-k A \frac{d T}{d r}=-k\left[2 \pi r^{2}(1-\cos \theta)\right] \frac{d T}{d r}
$$

The differential Equation (2.38) can be solved easily. Therefore, conduction heat transfer is defined as follows:

$$
q_{\text {Cond }}=\frac{-2 \pi(1-\cos \theta) k\left(T_{o}-T_{i}\right)}{1 / R_{i}-1 / R_{o}}
$$


The Nusselt number is found from rewriting the Equation 2.39 with the dimensionless variables and substituting it in the Equation 2.37. The Nusselt number takes the form:

$$
N u_{o}=\frac{q^{*}\left(R_{i}^{*}-R_{o}^{*}\right)}{2 \pi(1-\cos \theta) R_{o}^{*} R_{i}^{*} k\left(T_{o}^{*}-T_{i}^{*}\right)}
$$

where $q^{*}$ is the convection heat transfer from outer surface and will be calculated from numerical analysis.

Equation 2.40 is simplified by substituting the value of the dimensionless variables as follows:

$$
N u_{o}=\frac{q^{*}}{2 \pi(1-\cos \theta) R_{o}^{*}\left(R_{o}^{*}-1\right)}
$$

In this study the Nusselt number for uniform gap thickness is calculated from Equation 2.41.

Convection heat transfer for the problem in hand from the outer surface is:

$$
q_{o}=-\left.2 \pi R_{o}^{2} k \int_{0}^{\theta} \frac{\partial T}{\partial r}\right|_{r=R_{o}} \sin \theta d \theta
$$


Substituting Equation (2.39) and (2.42) into (2.37) and simplify the equation gives:

$$
N u_{o}=\left.\frac{R_{o}^{2}\left(1 / R_{o}-1 / R_{i}\right)}{(1-\cos \theta)\left(T_{o}-T_{i}\right)} \int_{0}^{\theta} \frac{\partial T}{\partial r}\right|_{r=R_{o}} \sin \theta d \theta
$$

To be consistent with the previous work of Laouadi and Atif (2001), the gap ratio is defined as:

$$
\delta=\frac{L}{R_{i}}
$$

From the dimensionless variables (Equation 2.27), one obtains:

$$
\begin{aligned}
& \partial T=\left(T_{o}-T_{i}\right) \partial T^{*} \\
& \partial r=L \partial r^{*}
\end{aligned}
$$

Substituting Equation 2.44 and 2.45 into Equation 2.43 gives:

$$
N u_{o}=\left.\frac{1+\delta}{(1-\cos \theta)} \int_{0}^{\theta} \frac{\partial T^{*}}{\partial r^{*}}\right|_{r^{*}=1 / \delta} \sin \theta d \theta
$$


It is practical to calculate the convection film in the steady-state condition, where the overall thermal conductance of the domed skylight can be calculated. The convection heat transfer from the inner surface to the outer surface is defined as:

$$
q=\frac{\left(A_{o}+A_{i}\right)}{2} h_{c}\left(T_{i}-T_{o}\right)
$$

where $A_{o}$ and $A_{i}$ are outer surface and inner surface respectively. Note that the surface area of the spherical cap is $A=2 \pi r^{2}(1-\cos \theta)$.

The Nusselt number takes the following form:

$$
N u=\frac{1+(1+\delta)^{2}}{2(1+\delta)} \frac{h_{c} L}{k}
$$

The convective film coefficient is thus given by:

$$
h_{c}=\frac{2(1+\delta)}{1+(1+\delta)^{2}} \frac{N u L}{k}
$$




\subsubsection{Cavities with Non-Uniform Gap Thickness}

The conduction heat transfer in non-uniform gap thickness domed cavities is no longer one-dimensional. To simplify the equations, the conduction heat transfer in non-uniform gap thickness cavity is estimated by uniform gap thickness domed cavity with the same truncation angle and outer radius. The equivalent gap thickness and inner radius are defined as:

$$
L=\frac{L_{\max }+L_{\min }}{2}, \quad R_{i}=R_{o}-L
$$

With the above assumption, the conduction heat transfer can be calculated from Equation 2.39. From dimensionless variables (Equation 2.27), one obtains:

$$
\partial r=L_{\max } \partial r^{*}
$$

With the above assumptions and following the same steps as uniform gap thickness, the Nusselt number is thus given by:

$$
N u_{o}=\left.\frac{\frac{1-d}{d \delta_{\max }^{\prime}}}{(1-\cos \theta)} \int_{0}^{\theta} \frac{\partial T^{*}}{\partial r^{*}}\right|_{r^{*}=1 / \delta^{\prime}} \sin \theta d \theta
$$

where $d$ can be found from Equation 2.9. 
The convective heat transfer from the outer surface should be calculated using the numerical procedure $\left(q^{*}\right)$ of the subsequent section.

\subsection{Transient Heat Transfer}

In some cases, for small gap to radius ratio the flow is transient periodic.

Consequently, the Nusselt number in such cases is periodic. Therefore, the Nusselt number was averaged over three or more oscillation periods. Figure 2.3 illustrates two periods of function $F(t)$ with time step size of $\Delta t$.

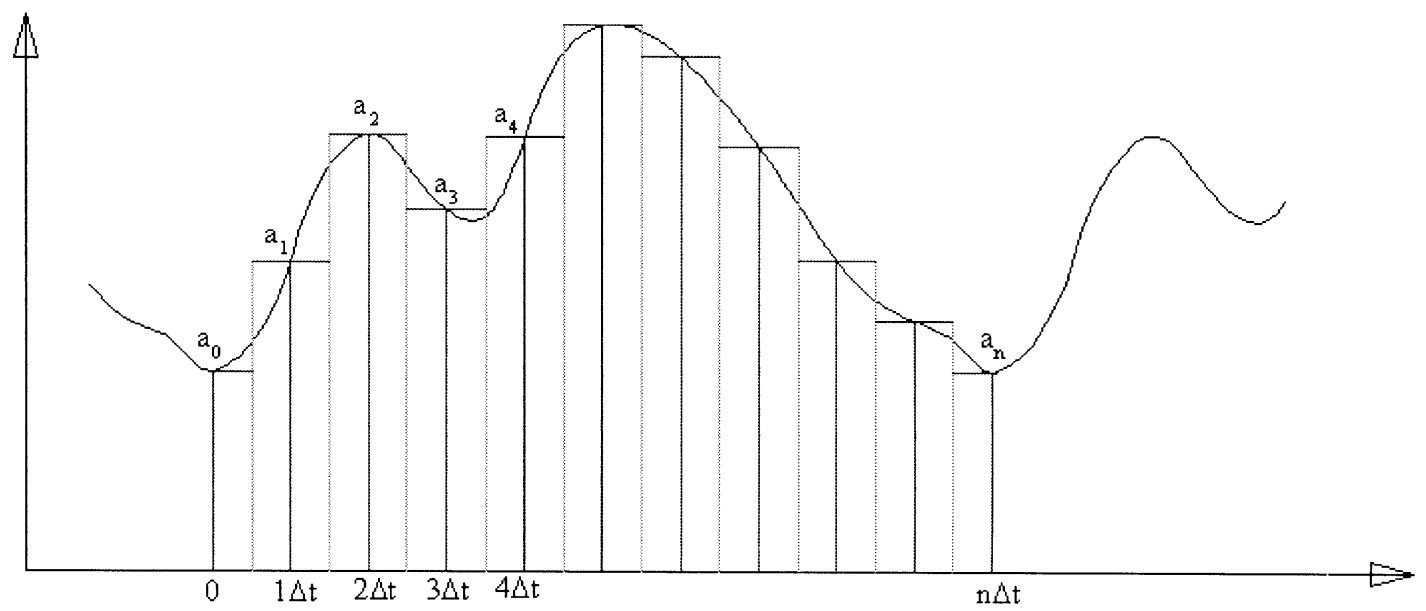

Figure 2.3: Oscillation of function $F(t)$ in time period when the time step is $\Delta t$. 
The time average of function $\mathrm{F}(\mathrm{t})$ in a time period is defined as follows:

$$
\bar{F}(t)=\frac{\int_{0}^{n \Delta t} F(t) d t}{n \Delta t-0}=\frac{\frac{a_{0} \Delta t}{2}+\sum_{1}^{n-1} a_{i} \Delta t+\frac{a_{n} \Delta t}{2}}{n \Delta t}=\frac{\sum_{0}^{n-1} a_{i}}{n}+\frac{a_{n}-a_{0}}{2 n}
$$

From Figure 2.3 it can be concluded that $\mathrm{a}_{\mathrm{n}}=\mathrm{a}_{0}$, since $\mathrm{a}_{\mathrm{n}}$ and $\mathrm{a}_{0}$ are the values for $\mathrm{a}$ period. As a result, Equation 2.53 is simplified as

$$
\bar{F}(t)=\frac{\sum_{0}^{n-1} a_{i}}{n}
$$

Equation 2.54 shows that the time averaged Nusselt number within three periods can be calculated by averaging the Nusselt number over those time periods.

\subsection{Table of Governing Parameters}

The dimensionless governing equations show that the free convective heat transfer in domed cavities is governed by several dimensionless parameters, including $\mathrm{Gr}$, $\operatorname{Pr}, \theta_{\mathrm{o}}, \delta_{\min }$ and $\delta_{\max }$. In this study, a wide range of parameter values are considered, except for Prandtl number.

Table 2.1 shows the values of the parameters. The gap to the inner radius ratio $(\delta)$ is used for the case of cavities with uniform gap thickness $\left(\varepsilon=0 \& \mathrm{~L}_{\max } / \mathrm{L}_{\min }=1\right)$ and the 
gap to outer radius $\left(\delta^{\prime}\right)$ is used for the case of cavity with non-uniform gap thickness $\left(\varepsilon \neq 0 \& \mathrm{~L}_{\max } / \mathrm{L}_{\min }=2\right)$.

Table 2.1: The value of the parameters

\begin{tabular}{ll}
\hline \multicolumn{1}{c}{ Parameter } & \multicolumn{1}{c}{ Value } \\
\hline $\operatorname{Pr}=$ & 0.72 \\
$L_{\max } / L_{\min }=$ & 1,2 \\
$\theta_{0}\left({ }^{\circ} \mathrm{C}\right)=$ & $90,45,30$ \\
$\delta\left(L^{\prime} / R_{i}\right)=$ & $1,0.8,0.5,0.4,0.3,0.2,0.1,0.01$ \\
$\delta^{\prime}\left(L_{\max } / R_{0}\right)\left(\theta_{0}=90^{\circ} \mathrm{C}\right)=$ & $0.5,0.4,0.3,0.25,0.2,0.15,0.1,0.05,0.025,0.015,0.01,0.008$ \\
$\delta^{\prime}\left(L_{\max } / R_{0}\right)\left(\theta_{0}=45^{\circ} \mathrm{C}\right)=$ & $0.4,0.35,0.3,0.25,0.2,0.15,0.1,0.05,0.025,0.015,0.01,0.009$ \\
$\delta^{\prime}\left(L_{\max } / R_{0}\right)\left(\theta_{0}=30^{\circ} \mathrm{C}\right)=$ & $0.2,0.15,0.1,0.05,0.025,0.015,0.01,0.010$ \\
$\mathrm{Gr}=$ & $1 \times 10^{3}, 2.5 \times 10^{3}, 5 \times 10^{3}, 7.5 \times 10^{3}, 1 \times 10^{4}, 2.5 \times 10^{4}, 5 \times 10^{4}, 7.5 \times 10^{4}$, \\
& $1 \times 10^{5}, 2.5 \times 10^{5}, 5 \times 10^{5}, 7.5 \times 10^{5}, 1 \times 10^{6}, 2.5 \times 10^{6}, 5 \times 10^{6}, 7.5 \times 10^{6}, 10^{7}$ \\
\hline
\end{tabular}




\section{CHAPTER 3}

\section{NUMERICAL APPROACH}

\subsection{Introduction}

It is usually very complicated and often impossible to find an analytical solution for convective heat transfer phenomenon. As a result, many numerical methods were developed to find a solution for a mathematical model. A numerical procedure for solving an equation will introduce uncertainty and truncation error to the results. Therefore, any study using a numerical approach to solve a problem must address truncation errors and other inaccuracies. Celik (1993) and Roache et al. (1986) hypothesized that the following conditions have a strong effect on accuracy:

1. Numerical method

2. Order of accuracy of discretized governing equations

3. Grid density

4. Grid topology

5. Convergence criteria

In the following sections, the above conditions are discussed and numerical results calculated from the present study are compared with available results to validate the numerical model. 


\subsection{Numerical Method, Parameters and Order of Accuracy}

In this study, a commercial computational fluid dynamics package, Fluent Version 6.2., employing the control volume approach was used to solve the laminar convective heat transfer within the cavity. The computational domain is divided into small volumes and the governing equations are numerically integrated over each control volume. Therefore, the governing equations are approximated by algebraic equations that could be solved iteratively. In order to increase the accuracy, the double-precision version of Fluent was used in analysis. For all simulations the axisymmetric option of FLUENT was selected. The segregated solver along with implicit formulation, which provides better stability than explicit method Anderson (1995), was used to linearize the governing equations.

As per the discussion in the previous chapter, the dimensionless governing equations should be used to find the general solution of heat transfer in domed skylights. Since the FLUENT software solves dimensional governing equations, the following steps describe how to setup the dimensionless governing equations in FLUENT. FLUENT solves the governing equation 2.27 to 2.30 over the computational domain using the control volume method. By comparing the dimensionless governing equations (Equation 2.28 to 2.31 ) with dimensional governing equations (Equation 2.15, 2.18, 2.21 and 2.22), it is concluded that they are identical and only the coefficients of the equations are different. Therefore, it can be concluded that:

$$
\rho \equiv k_{f} \equiv \mu \equiv \beta \equiv 1, \quad g \equiv G r, \quad c_{P} \equiv \operatorname{Pr}, \quad T_{\text {hot }}=1, \quad T_{\text {cold }}=0
$$


As a result, for each type of geometry, if the fluid properties in FLUENT are set up using Equation 3.1, the general dimensionless result may be found. Gravity in the numerical model represents the Grashof number in dimensionless equations. Therefore, only the gravity value in the numerical solver is changed to vary the Rayleigh number. The geometry is non-dimensionalized by dividing all dimensions by $\mathrm{L}_{\max }$ which is used as the characteristic length. Therefore, it can be concluded that:

$$
L_{\max }^{*}=\frac{L_{\max }}{L_{\max }}=1, \quad L_{\min }^{*}=\frac{L_{\min }}{L_{\max }}, \quad R_{i}^{*}=\frac{R_{i}}{L_{\max }}, \quad R_{i}^{*}=\frac{R_{o}}{L_{\max }}
$$

It is useful to characterize the domed geometries to generalize the solution. Therefore, the following gap-to-radius ratios are defined:

$$
\delta=\frac{L_{\max }}{R_{i}}, \quad \delta^{\prime}=\frac{L_{\max }}{R_{o}}
$$

Since the flow is assumed to be laminar, the laminar flow option was used in FLUENT. The SIMPLEC algorithm was selected for the solution control. The SIMPLEC algorithm uses a relationship between velocity and pressure correction to enforce the mass conservation (Fluent 6.2 Documentation). More details on the SIMPLEC algorithm can be found in Versteeg and Malalasekera (1995). For pressure discretization the PRESTO! scheme was used. This scheme is described in detail in Versteeg and Malalasekera (1995). The PRESTO! scheme is recommended for natural convection flow 
with high Rayleigh numbers in the FLUENT 6.2 documentation. The second order upwind discretization scheme is used in the momentum and energy equations to increase the accuracy of the results. Although a first order discretization scheme yields better convergence than second order scheme, nevertheless the first order can increase the numerical truncation error (FLUENT 6.2 documentation).

The under-relaxation factors for the density, body forces and energy were usually set to the value of one, while the under-relaxation factor for pressure and momentum were set to 0.3 and 0.7 respectively. However, in this study for geometries with a small gap-to-radius ratio and high Rayleigh number, the values of the under-relaxation factor were decreased to achieve numerical convergence.

The steady-state solver was used to solve the problems with large gap-to-radius ratio cavities. In the small gap-to-radius ratio cavities, the results show that the flow is unsteady. Therefore, for the small gap cavities the unsteady-state option was selected along with a unit time step size. Also, a smaller time step size was used as a check, to make sure that the time step is small enough to capture the periodic behaviour of the flow and heat transfer.

\subsection{Boundary and Initial Conditions}

For the problem in hand, which is natural convection heat transfer in a closed cavity, the interior and exterior surfaces of the cavity have Dirichlet boundary conditions. 
This means that the interior and exterior surfaces of the domed skylight cavity have constant and uniform temperature. It is assumed that the edge of domed cavity is adiabatic, which is a Neumann boundary condition. For the initial condition, the fluid is assumed quiescent, therefore the fluid velocity is zero and there is no heat transfer by convection at the initial condition. The temperature at the initial condition is set to the value of one, which is equal to the temperature of the hot boundary surface. These initial conditions were used for all simulations, although the initial conditions were not required for steady-state solutions.

\subsection{Grid Topology and Grid Density}

The quality of grid has a significant effect on the accuracy and stability of the numerical results. The quality of grid consists of the grid topology and grid density. The grid topology plays an important role in truncation error (numerical diffusion). Numerical diffusion can be reduced by choosing a proper grid topology, which is aligned with the fluid flow. For natural convection in domed cavities, when the gap thickness is small relative to the domed radius, the flow is mainly along the boundary surfaces. Therefore, a quadrilateral grid type should be aligned with the fluid flow in order to reduce numerical diffusion. Grid density is the other important factor in numerical accuracy. A finer grid should be used where there is a high thermal gradient or high velocity gradient in the computational domain. 
For the problem in hand, the thermal and velocity gradients are high near the interior and exterior surfaces. As a result, a finer grid was used near the exterior and interior surfaces to increase numerical accuracy. This study covers a wide range of domed skylight geometries. Therefore, for each geometry, the results with different mesh sizes were compared to find the proper mesh size that gave grid independent results.

Figure 3.1 shows a partial section of a quadrilateral grid in a concentric spherical cap cavity when the truncation angle is $30^{\circ}$ degree and gap-to-radius ratio is equal to the value of $0.1(\delta=0.1)$. Note that the grid density increases near the interior and exterior surfaces.



Figure 3.1: Section of a quadrilateral grid for $\theta=30^{\circ}, \varepsilon=0$ and $\delta=0.1$ 
Table 3.1 shows the comparison between Nusselt number with four different grid sizes in a concentric spherical cavity with the truncation angle of $30^{\circ}$ degree and gap-to-radius ratio of $\delta=0.1$. Results are shown for a Rayleigh number of $7.5 \times 10^{3}$, $5 \times 10^{4}$ and $5 \times 10^{5}$. The Nusselt numbers are calculated for the condition when the interior surface is hotter than the exterior one. The percentage change in Table 3.1 is calculated from the following equation:

$$
\% \text { Difference }=\frac{\left|N u_{\text {old }}-N u_{\text {new }}\right|}{N u_{\text {new }}} \times 100
$$

Table 3.1: Comparison of Nusselt number with different grid density. $\left(\theta_{0}=30^{\circ}, \mathrm{T}_{0}<\mathrm{T}_{\mathrm{i}}\right.$, $\delta=0.1$ and $\varepsilon=0$ )

\begin{tabular}{|c|c|c|c|c|c|c|}
\hline \multirow{2}{*}{ Grid } & \multicolumn{2}{|c|}{ Ra $=\mathbf{7 . 5 \times 1 0}^{\mathbf{3}}$} & \multicolumn{2}{c|}{ Ra $=\mathbf{5 \times 1 0} \mathbf{4}^{\mathbf{4}}$} & \multicolumn{2}{c|}{ Ra $=\mathbf{5 \times 1 0} \mathbf{5}^{\mathbf{5}}$} \\
\cline { 2 - 7 } & Nu & \% Change & Nu & \% Change & Nu & \% Change \\
\hline $40 \times 30$ & 2.0622 & & 3.5125 & & 5.9458 & \\
\hline $50 \times 40$ & 2.0675 & $0.256 \%$ & 3.5357 & $0.66 \%$ & 6.0816 & $2.23 \%$ \\
\hline $60 \times 50$ & 2.0657 & $0.087 \%$ & 3.5459 & $0.29 \%$ & 5.7584 & $5.61 \%$ \\
\hline $70 \times 60$ & 2.0639 & $0.085 \%$ & 3.5467 & $0.02 \%$ & 5.7530 & $0.09 \%$ \\
\hline
\end{tabular}

It can be concluded from Table 3.1 that for a relatively small Rayleigh numbers, there is a small effect on Nusselt number due to increasing the grid density. Therefore, the results with a relatively coarse grid might be accurate for small Rayleigh numbers. However, when the Rayleigh number is high, the grid density has a significant effect on 
Nusselt numbers. Therefore, in order to obtain grid independent results, the numerical results for high Rayleigh number with different mesh sizes should be compared to find the proper mesh size for each geometry. In this study, the grid density was increased, until the percentage difference between the Nusselt numbers, calculated with the last two grids, was less than $0.1 \%$ for the Rayleigh numbers of $7.5 \times 10^{3}, 5 \times 10^{4}$ and $5 \times 10^{5}$.

\subsection{Accuracy and Convergence Criteria}

In a numerical study, it is difficult to separate the numerical errors from modeling errors. The first effort in any numerical study is having an accurate mathematical model and then trying to solve this model numerically. There are a few methods to examine numerical accuracy and convergence. But, unfortunately there is no universal method to determine numerical convergence. Depending on the problem, there are different criteria that play a main role in numerical convergence and accuracy. Generally, the residuals for continuity, $x$ and $y$ momentum and energy equations are considered as convergence criteria. In this study, in addition to those criteria, the heat transfer from the exterior

surface was monitored as well. The convergence criteria were set to $10^{-3}$ for the residuals of continuity, $x$ and $y$ momentum and energy, and include changes in heat transfer from the exterior surface (Equation 3.5) less than $10^{-3}$. The change in heat transfer rate is defined as follows:

$$
\frac{q^{*(i-1)}-q^{*(i)}}{q^{*(i)}}<10^{-3}
$$


where $(i)$ is the iteration level.

For small gap-to-radius ratios $(\delta<0.2$ or $\delta$ ' $<0.15)$, the flow might become unsteady. Therefore, the simulation was run in the unsteady state mode. In these cases, at each time step the convergence criteria should be satisfied. The same convergence criteria were considered for each time step in the unsteady flow.

\subsection{Flow Stability and Critical Rayleigh Number}

When the Rayleigh number is increased in natural convection in spherical cavities, at some point the flow will become transient or turbulent. Therefore, laminar flow could under-predict the heat transfer rate. In such cases, the flow is unstable and the dimensionless velocity is too high to satisfy the convergence criteria. As a result, the convergence criteria was changed to $10^{-1}$. This will be discussed in more detail in the next chapter.

There is some information available about the fluid flow and the critical Rayleigh number in spherical cavities. But, to the knowledge of the author, there is no information available regarding the critical Rayleigh number when the flow is in transition to the turbulent regime in spherical cap cavities. The Rayleigh numbers that cause unstable flow are reported in the next chapter. 


\subsection{Numerical Model Validation}

To the knowledge of author, there are no experimental or numerical results available for heat transfer within spherical cap cavities, except the one by Laouadi and Atif (2001). However, there are many studies available for other related geometries such concentric spheres $\left(\theta_{0}=180^{\circ}\right)$. The latter studies on heat transfer in concentric spheres are used to validate present model. In addition, the numerical results for heat transfer in dome cavities from present mode are compared with the one from Laouadi and Atif (2001).

For concentric sphere cavities, Raithby and Hollands (1975) employed a thin boundary layer approximation to study natural laminar convection within spherical cavities which are heated from inside. They suggested the following equation to find the Nusselt number within two concentric spheres when the interior sphere is hotter than the exterior one.

$$
N u=\max \left\{1, c\left(R a^{*}\right)^{1 / 4}\right\}
$$

where $c$ is a coefficient, which is calculated from the experimental data, and $\mathrm{Ra}^{*}$ is a modified Rayleigh number. The coefficient $c$ and modified Rayleigh number (Ra*) are defined as follows: 


$$
\begin{aligned}
& R a^{*}=\frac{R a(\delta / 2)}{\left\{(1+\delta)^{-3 / 5}+(1+\delta)^{4 / 5}\right\}^{5}} \\
& c=0.74\left(\frac{\operatorname{Pr}}{0.861+\operatorname{Pr}}\right)^{1 / 4}
\end{aligned}
$$

The results from Equation 3.6 match the experimental data within $13 \%$. Table 3.2 shows the Nusselt number calculated for concentric spheres, when heated from inside, using Equation 3.6 and the present model. In Table 3.2 the Nusselt numbers were calculated for concentric sphere cavities with $\delta=1$ when air filled the gap $(\operatorname{Pr}=0.7)$. From Table 3.2 it can be concluded that the present model over predicts the results by a maximum error of $13 \%$, which is within the reported error of Equation (3.6).

Table 3.2: Nusselt number comparison between present model and Raithby and Hollands (1975) in spherical cavities $\left(\varepsilon=0, \theta_{0}=180^{\circ}, \delta=1\right.$ and $\left.\mathrm{T}_{\mathrm{o}}<\mathrm{T}_{\mathrm{i}}\right)$

\begin{tabular}{cccc}
\hline Ra & $\begin{array}{c}\text { Present Model } \\
\left(\mathbf{N u}_{\mathbf{L}}\right)\end{array}$ & $\begin{array}{c}\text { Raithby \& } \\
\text { Hollands } \\
\left(\mathbf{N u}_{\mathbf{L}}\right)\end{array}$ & \% Difference \\
\hline 1000 & 1.1000 & 1.0000 & $9.09 \%$ \\
3000 & 1.4180 & 1.2610 & $11.07 \%$ \\
6300 & 1.7346 & 1.5180 & $12.49 \%$ \\
10500 & 1.9800 & 1.7248 & $12.89 \%$ \\
14000 & 2.1283 & 1.8534 & $12.92 \%$ \\
21000 & 2.3511 & 2.0511 & $12.76 \%$ \\
42000 & 2.7707 & 2.4392 & $11.96 \%$ \\
91000 & 3.3030 & 2.9594 & $10.42 \%$ \\
\hline
\end{tabular}


Grag (1991) and Laouadi and Atif (2001) calculated the Nusselt number for concentric spheres. They all used numerical approaches to find the Nusselt number when the interior surface is hotter than exterior one and the gap is equal to the inner radius $(\delta=1)$. The Nusselt number calculated from their numerical models and present the model are shown in Table 3.3 and Table 3.4 respectively.

From Table 3.3 and 3.4, it can be concluded that the Nusselt number calculated from the present model shows a good agreement with the results from Grag (1991) and Laouadi and Atif (2001). The maximum percentage difference is less than $1 \%$ in both cases.

Table 3.3: Nusselt number comparison between present model and Grag (1991) in spherical cavities $\left(\varepsilon=0, \theta_{0}=180^{\circ}, \delta=1\right.$ and $\left.\mathrm{T}_{0}<\mathrm{T}_{\mathrm{i}}\right)$

\begin{tabular}{cccc}
\hline Ra & $\begin{array}{c}\text { Present Model } \\
\left(\mathbf{N u}_{\mathbf{L}}\right)\end{array}$ & $\begin{array}{c}\mathbf{G r a g} \\
\left(\mathbf{N u}_{\mathbf{L}}\right)\end{array}$ & \% Difference \\
\hline 1000 & 1.1000 & 1.1006 & $0.06 \%$ \\
3000 & 1.4180 & 1.4213 & $0.24 \%$ \\
6300 & 1.7346 & 1.7393 & $0.27 \%$ \\
10500 & 1.9800 & 1.9848 & $0.24 \%$ \\
14000 & 2.1283 & 2.1331 & $0.23 \%$ \\
21000 & 2.3511 & 2.3560 & $0.21 \%$ \\
42000 & 2.7707 & 2.7761 & $0.19 \%$ \\
91000 & 3.3030 & 3.3110 & $0.23 \%$ \\
\hline
\end{tabular}


Table 3.4: Nusselt number comparison between present model and Laouadi (2001) in spherical cavities $\left(\varepsilon=0, \theta_{0}=180^{\circ}, \delta=1\right.$ and $\left.\mathrm{T}_{0}<\mathrm{T}_{\mathrm{i}}\right)$

\begin{tabular}{cccc}
\hline Ra & $\begin{array}{c}\text { Present Model } \\
\left(\mathbf{N u}_{\mathbf{L}}\right)\end{array}$ & $\begin{array}{c}\text { Laouadi and Atif } \\
(\mathbf{2 0 0 1}) \\
\left(\mathbf{N u}_{\mathbf{L}}\right)\end{array}$ & \% Difference \\
\hline 1000 & 1.1000 & 1.100 & $0.00 \%$ \\
3000 & 1.4180 & 1.420 & $0.14 \%$ \\
6300 & 1.7346 & 1.737 & $0.14 \%$ \\
10500 & 1.9800 & 1.980 & $0.00 \%$ \\
14000 & 2.1283 & 2.127 & $0.06 \%$ \\
21000 & 2.3511 & 2.345 & $0.26 \%$ \\
42000 & 2.7707 & 2.760 & $0.39 \%$ \\
91000 & 3.3030 & 3.283 & $0.62 \%$ \\
\hline
\end{tabular}

Laouadi and Atif (2001) studied natural laminar convection within concentric spherical cap cavities with the truncation angles of $30^{\circ}, 45^{\circ}$ and $90^{\circ}$ when heated from outside $\left(T_{0}>T_{i}\right)$ with an air filled the gap $(\operatorname{Pr}=0.7)$. They suggested the following equations to find the Nusselt number:

$$
N u=\max \left\{1, c\left(R a^{*}\right)^{d / 4}\right\}
$$

where $c$ and $d$ are coefficients that were found from curve fitting the numerical results. The coefficients are defined as follows: 
For $\theta_{0}=90^{\circ}$;

$$
\begin{aligned}
& d=1 \\
& c=0.7943-0.2461 \delta+0.1129 \delta^{2}-0.0162 \delta^{3}
\end{aligned}
$$

For $\theta_{0}=45^{\circ}$;

$$
\begin{aligned}
& d=\frac{1-3.0346 \delta+4.1542 \delta^{2}}{1-2.5071 \delta+2.27 \delta^{2}+8.349 \delta^{3}} \\
& c=\frac{0.8439+0.8375 \delta-17.7074 \delta^{2}+49.736 \delta^{3}}{1+2.4185 \delta-24.1328 \delta^{2}+58.2117 \delta^{3}}
\end{aligned}
$$

For $\theta_{0}=30^{\circ}$;

$$
\begin{aligned}
& d=\frac{1.0178-7.1552 \delta+18.6979 \delta^{2}}{1-5.5826 \delta+9.3132 \delta^{2}+123.4 \delta^{3}} \\
& c=\frac{0.8367-9.8836 \delta+50.133 \delta^{2}}{1-9.598 \delta+39.6426 \delta^{2}+26.0183 \delta^{3}}
\end{aligned}
$$

The Nusselt numbers calculated from the present model are compared with the Nusselt numbers calculated from Equation 3.8. Table 3.5 and 3.6 shows the comparison between the two Nusselt numbers for a truncation angle $\left(\theta_{0}\right)$ of $45^{\circ}$ with gap-to-radius ratio $(\delta)$ of 0.5 and for the truncation angle of $90^{\circ}$ with gap-to-radius ratio $(\delta)$ of 0.2 . 
Table 3.5: Nusselt number comparison between present model and Equation 3.8 $\left(\varepsilon=0, \theta_{0}=45^{\circ}, \delta=0.5\right.$ and $\left.\mathrm{T}_{0}>\mathrm{T}_{\mathrm{i}}\right)$

\begin{tabular}{cccc}
\hline Gr & $\begin{array}{c}\text { Present Model } \\
(\mathbf{N u})\end{array}$ & $\begin{array}{c}\text { Laouadi and } \\
\text { Atif (2001) } \\
(\mathbf{N u})\end{array}$ & \% Difference \\
\hline $1.0 \mathrm{E}+03$ & 1.0205 & 1.0042 & $1.59 \%$ \\
$2.5 \mathrm{E}+03$ & 1.0718 & 1.0965 & $2.30 \%$ \\
$5.0 \mathrm{E}+03$ & 1.1445 & 1.1720 & $2.40 \%$ \\
$7.5 \mathrm{E}+03$ & 1.1981 & 1.2185 & $1.70 \%$ \\
$1.0 \mathrm{E}+04$ & 1.2394 & 1.2526 & $1.07 \%$ \\
$2.5 \mathrm{E}+04$ & 1.3789 & 1.3678 & $0.81 \%$ \\
$5.0 \mathrm{E}+04$ & 1.4872 & 1.4619 & $1.70 \%$ \\
$7.5 \mathrm{E}+04$ & 1.5512 & 1.5199 & $2.02 \%$ \\
$1.0 \mathrm{E}+05$ & 1.5972 & 1.5625 & $2.17 \%$ \\
$2.5 \mathrm{E}+05$ & 1.7457 & 1.7061 & $2.27 \%$ \\
$5.0 \mathrm{E}+05$ & 1.8606 & 1.8235 & $2.00 \%$ \\
$7.5 \mathrm{E}+05$ & 1.9289 & 1.8959 & $1.71 \%$ \\
$1.0 \mathrm{E}+06$ & 1.9779 & 1.9489 & $1.46 \%$ \\
\hline
\end{tabular}

Table 3.5 shows that the Nusselt numbers calculated from the present model matches within $3 \%$ with the Nusselt numbers calculated from Equation 3.8. The Nusselt numbers for the same truncation angle with some other gap-to-radius ratios $(\delta)$ and different Rayleigh numbers were compared as well. The percentage differences in all of the compared values are less than $5 \%$. 
Table 3.6: Nusselt number comparison between present model and Equation 3.8 $\left(\varepsilon=0, \theta_{0}=90^{\circ}, \delta=0.2\right.$ and $\left.\mathrm{T}_{\mathrm{o}}>\mathrm{T}_{\mathrm{i}}\right)$

\begin{tabular}{cccc}
\hline $\mathbf{G r}$ & $\begin{array}{c}\text { Present Model } \\
(\mathbf{N u})\end{array}$ & $\begin{array}{c}\text { Laouadi and } \\
\text { Atif (2001) } \\
(\mathbf{N u})\end{array}$ & \% Difference \\
\hline $1.0 \mathrm{E}+03$ & 1.0280 & 1.0000 & $2.73 \%$ \\
$2.5 \mathrm{E}+03$ & 1.1205 & 1.1168 & $0.33 \%$ \\
$5.0 \mathrm{E}+03$ & 1.2844 & 1.3281 & $3.40 \%$ \\
$7.5 \mathrm{E}+03$ & 1.4252 & 1.4698 & $3.13 \%$ \\
$1.0 \mathrm{E}+04$ & 1.5424 & 1.5794 & $2.40 \%$ \\
$2.5 \mathrm{E}+04$ & 1.9905 & 1.9860 & $0.22 \%$ \\
$5.0 \mathrm{E}+04$ & 2.4009 & 2.3617 & $1.63 \%$ \\
$7.5 \mathrm{E}+04$ & 2.6629 & 2.6137 & $1.85 \%$ \\
$1.0 \mathrm{E}+05$ & 2.8594 & 2.8086 & $1.78 \%$ \\
$2.5 \mathrm{E}+05$ & 3.5769 & 3.5316 & $1.27 \%$ \\
$5.0 \mathrm{E}+05$ & 4.2385 & 4.1998 & $0.91 \%$ \\
$7.5 \mathrm{E}+05$ & 4.6815 & 4.6479 & $0.72 \%$ \\
$1.0 \mathrm{E}+06$ & 5.0240 & 4.9945 & $0.59 \%$ \\
$2.5 \mathrm{E}+06$ & 6.2946 & 6.2802 & $0.23 \%$ \\
$5.0 \mathrm{E}+06$ & 7.4673 & 7.4685 & $0.02 \%$ \\
$7.5 \mathrm{E}+06$ & 8.2562 & 8.2653 & $0.11 \%$ \\
$1.0 \mathrm{E}+07$ & 8.8729 & 8.8816 & $0.10 \%$ \\
\hline
\end{tabular}

The comparison between the Nusselt numbers calculated from two different models for a truncation angle of $90^{\circ}$ shows that the maximum percentage difference is $3.4 \%$. For the same truncation angle, some other Nusselt numbers for different gap-to-radius ratio $(\delta)$ and Rayleigh numbers were compared and the overall percentage difference was less than $5 \%$. 


\subsection{Summary}

By selecting the proper options and methods for the numerical procedure, which was discussed in Section 3.1, and the proper grid type and grid size, the numerical errors can be reduced. The comparison between the results from the present model and the results from other researchers confirms that the numerical model is valid and has been set up properly. In the following chapter, the numerical results from the present model will be discussed. 


\section{CHAPTER 4}

\section{RESULTS AND DISCUSSION}

\subsection{Introduction}

This chapter presents the numerical results for two different types of geometries of domed cavities: concentric and eccentric geometries. Given the parameter ranges within Table 2.1, a total of 1400 cases were simulated.

\subsection{Results for Domed Concentric Cavities}

The results for the laminar natural convection in domed concentric cavities with uniform gap spacing $\left(\varepsilon=0\right.$ or $\left.\mathrm{L}_{\max } / \mathrm{L}_{\min }=1\right)$ are presented for the case where the interior surface temperature is greater than the exterior one $\left(T_{i}>T_{0}\right)$. The results cover truncation angles of $90^{\circ}, 45^{\circ}$ and $30^{\circ}$ with varying cavity gap thickness.

\subsubsection{Concentric Cavities with Truncation Angle $\left(\theta_{0}\right)$ of $90^{\circ}$}

At large gap-to-radius ratios $(0.5 \leq \delta \leq 1)$, the numerical results reached steady state conditions for all Grashof numbers in the range of this study. The results for large gap cavities showed that the flow is made of a single vortex-cell. Streamlines and Isotherms are shown in Figures 4.1 to 4.3 for $\delta=0.8$ while the Grashof number is $1 \times 10^{3}$, 
$5 \times 10^{4}$ and $5 \times 10^{6}$. From Figure 4.1 to 4.3 , it can be concluded that increasing the Grashof number causes the vortex-cell to become bigger.
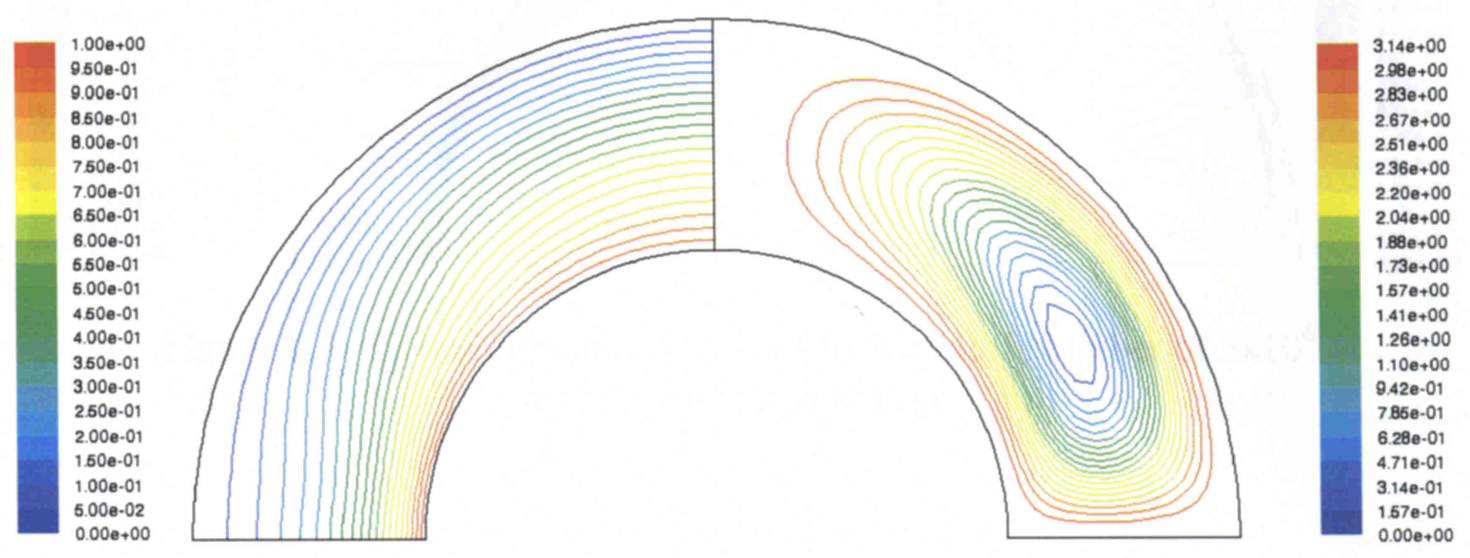

Figure 4.1: Streamlines and isotherms for Grashof Number of $1 \times 10^{3}$ $\left(\theta_{0}=90^{\circ}, \varepsilon=0\right.$ and $\left.\delta=0.8\right)$

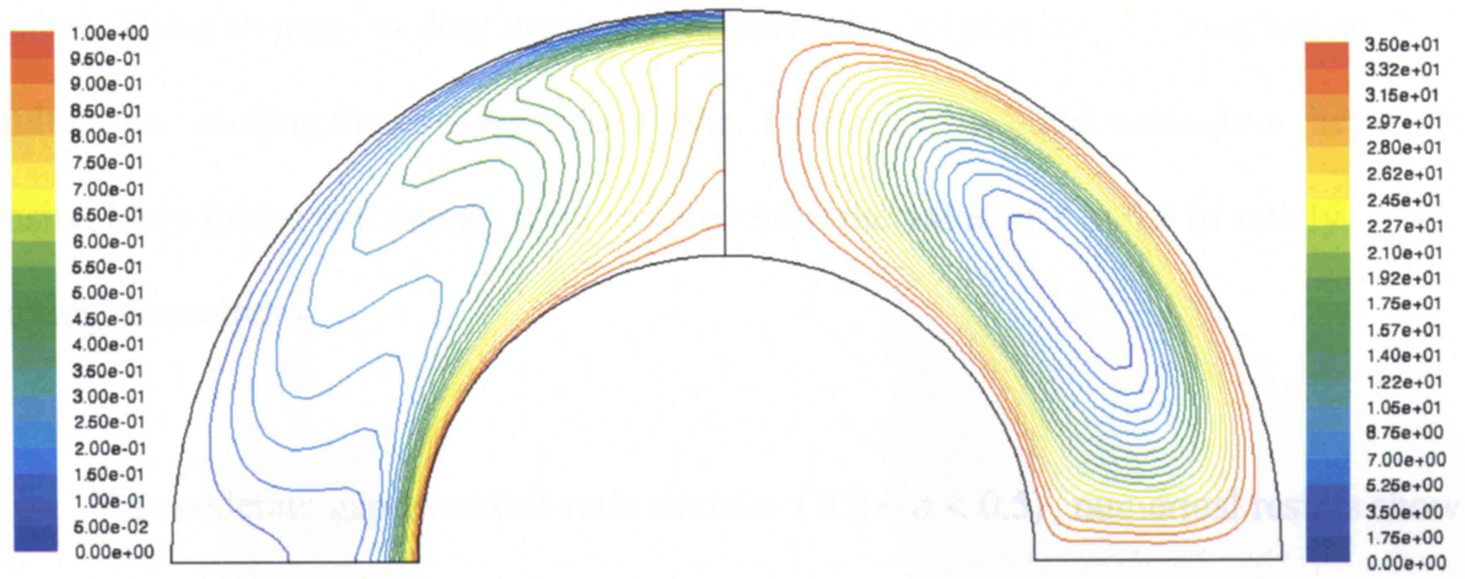

Figure 4.2: Streamlines and isotherms for Grashof Number of $5 \times 10^{4}$ $\left(\theta_{0}=90^{\circ}, \varepsilon=0\right.$ and $\left.\delta=0.8\right)$ 


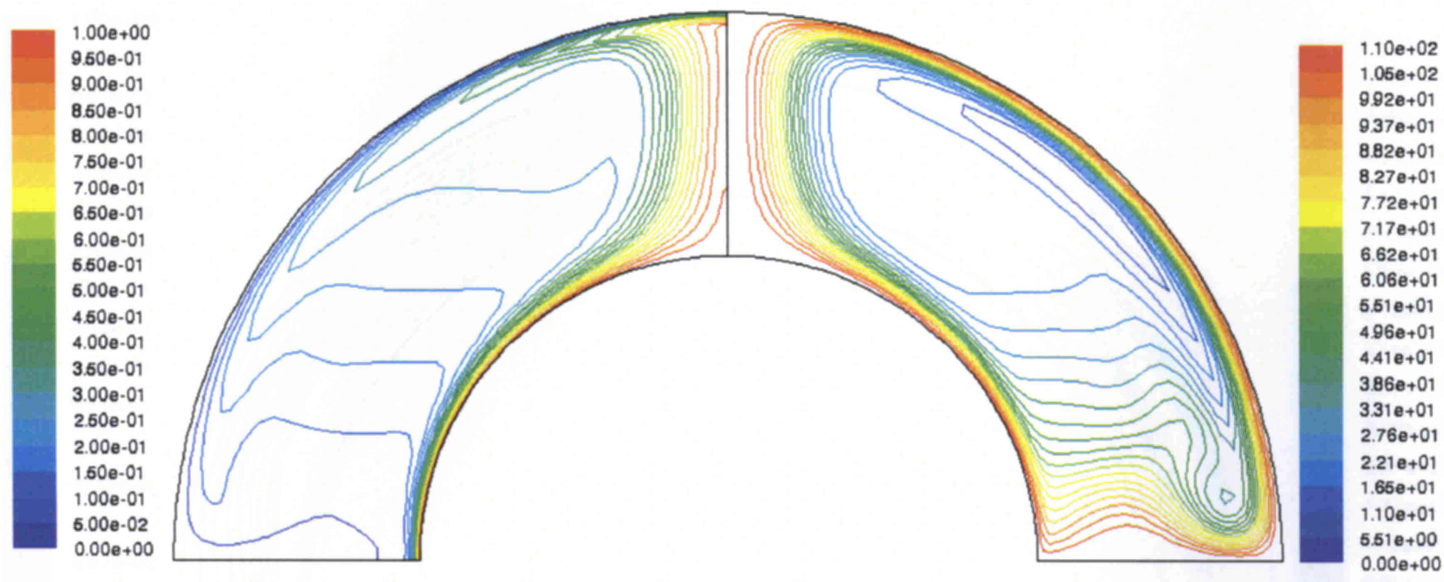

Figure 4.3: Streamlines and isotherms for Grashof Number of $5 \times 10^{6}$ $\left(\theta_{0}=90^{\circ}, \varepsilon=0\right.$ and $\left.\delta=0.8\right)$

Figure 4.3 shows that a small vortex-cell forms inside the main vortex-cell in the bottom region. This behavior was found for all large gap cavities at high Grashof numbers, except for $\delta=1$. In addition to a small vortex-cell, weak changes in flow were observed inside the big vortex-cell, which caused a very small oscillation in the Nusselt number. These changes in flow increased the residuals and prevented the numerical results from reaching the convergence criteria. Therefore, the under-relaxation factors for density, body forces and energy were reduced from the value of 1 to 0.9 to satisfy convergence criteria.

For moderate gap-to-radius ratio cavities $(0.2<\delta<0.5)$, numerical results show that the flow is in the steady state region, with a single-vortex-cell or multi-vortex-cells. Figures 4.4 to 4.6 present the streamlines and isotherms for $\delta=0.3$ at three Grashof numbers of $1 \times 10^{3}, 5 \times 10^{4}$ and $5 \times 10^{6}$. 


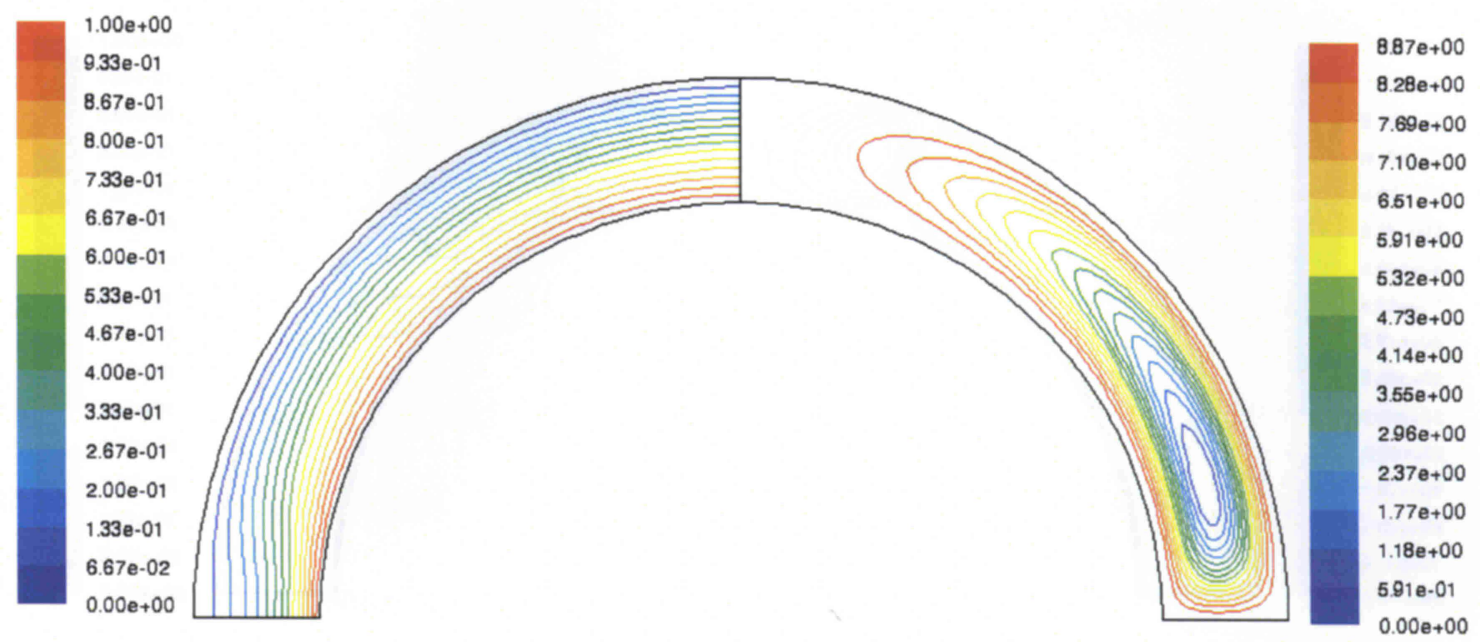

Figure 4.4: Streamlines and isotherms for Grashof Number of $1 \times 10^{3}$ $\left(\theta_{0}=90^{\circ}, \varepsilon=0\right.$ and $\left.\delta=0.3\right)$

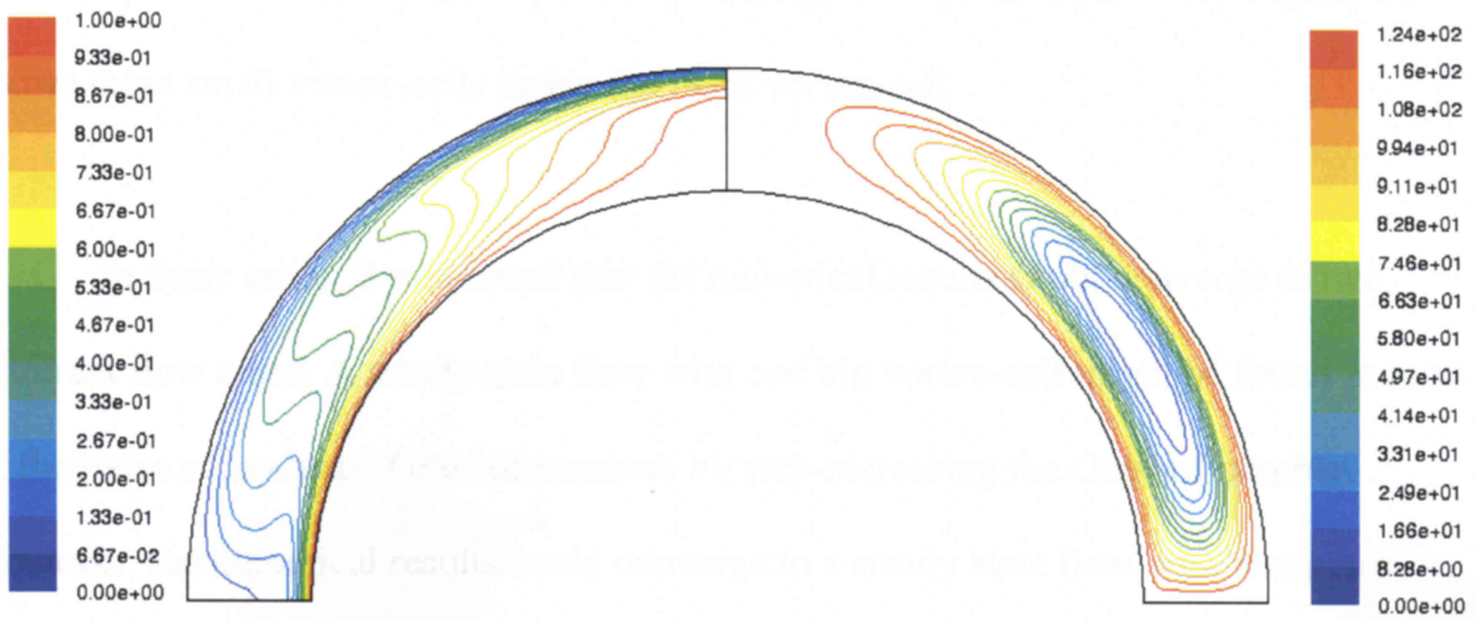

Figure 4.5: Streamlines and isotherms for Grashof Number of $5 \times 10^{4}$ $\left(\theta_{0}=90^{\circ}, \varepsilon=0\right.$ and $\left.\delta=0.3\right)$ 




Figure 4.6: Streamlines and isotherms for Grashof Number of $5 \times 10^{6}$ $\left(\theta_{0}=90^{\circ}, \varepsilon=0\right.$ and $\left.\delta=0.3\right)$

When the Grashof number is greater than $10^{6}$, small vortex-cells form inside the main vortex-cell. These vortex-cells move inside the main vortex-cell, which causes small oscillations in heat transfer, and consequently in the Nusselt number. Figure 4.6 shows these small vortex-cells inside the main vortex-cell.

In some cases, it was found that the numerical results could converge to two different flow types. A steady state flow with one big vortex-cell might be found in most of the cases at moderate Grashof numbers by step-increasing the Grashof number. However, the numerical results could converge to a steady state flow with two main vortex-cells, if the results were calculated by step-decreasing Grashof number. The Navier-Stokes equations are nonlinear and nonlinear equations can have multiple solutions. One or more may be non-physical and experiments are needed to determine this. 
The heat transfer rate with two vortex-cells is higher than the one with one vortex-cell. For instance, when the Grashof number is $5 \times 10^{4}$ and $\delta=0.5$, the Nusselt number calculated by step-decreasing the Grashof number (two-vortex-cells) is $6.16 \%$ higher then the Nusselt number calculated by step-increasing the Grashof number (one-vortex-cell). In this study, the Nusselt numbers were calculated by step-increasing the Grashof number. Figure 4.7 shows two different types of steady flows that were found by step-increasing and step-decreasing the Grashof number.

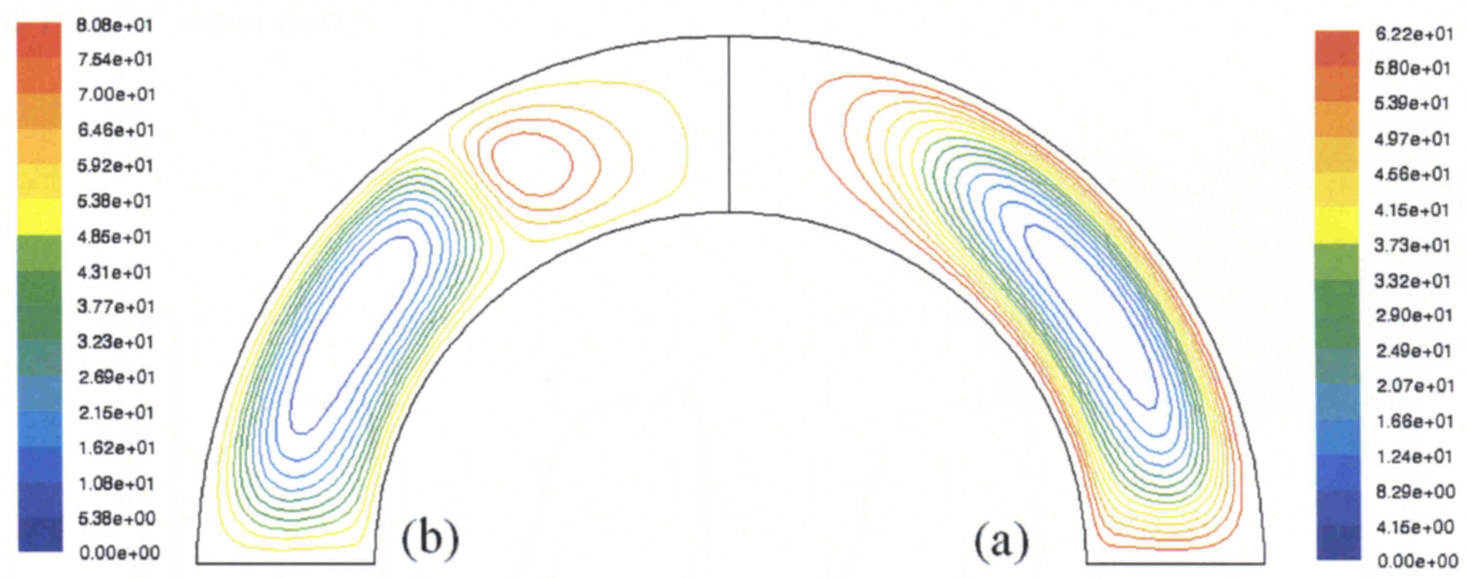

Figure 4.7: Streamlines for Grashof Number of $5 \times 10^{4}\left(\theta_{0}=90^{\circ}, \varepsilon=0\right.$ and $\left.\delta=0.5\right)$ a) Step-increasing Grashof number b) Step-decreasing Grashof Number

For small gap-to-radius ratios $(\delta \leq 0.2)$, steady state flows were observed for Grashof numbers smaller than $5 \times 10^{3}$. In this range of Grashof numbers, the fluid flow is made of a single vortex-cell. Unsteady flows with multi-vortex-cells were found for 
Grashof numbers in the range of $5 \times 10^{3} \leq G r \leq 5 \times 10^{4}$. In this range of Grashof numbers, small vortex-cells formed at the top of the cavity, along with a bigger vortex-cell at the bottom. Small vortex-cells, which were formed on top of the cavity, were pushed down by the newly formed vortex-cells and then merge with the big main vortex-cell. This behavior resulted in an oscillation in the Nusselt number. The numerical results showed unsteady flow. Nevertheless, the changes in flow were repeated periodically, which caused a periodic change in the Nusselt number. The Nusselt numbers for such cases were calculated by averaging the Nusselt number over three or more oscillation periods in time. Figure 4.8 shows such an oscillation in the Nusselt number for a Grashof number of $7.5 \times 10^{3}$ when $\delta=0.2$.

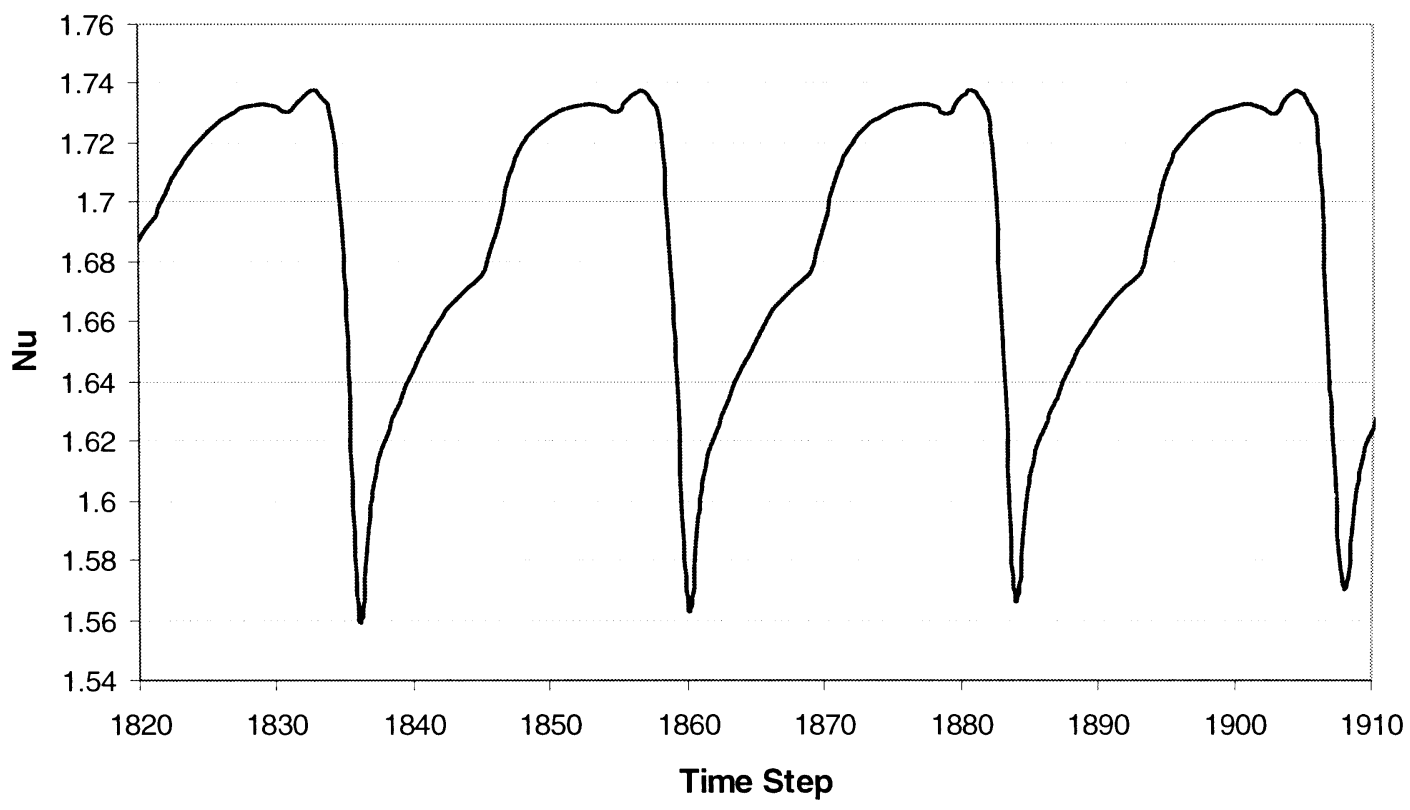

Figure 4.8: Changes in Nusselt number in time (Time Step $=0.5 \mathrm{~s}$ ) when $\mathrm{Gr}=7.5 \times 10^{3}$ $\left(\theta_{0}=90^{\circ}, \varepsilon=0\right.$ and $\left.\delta=0.2\right)$ 
Figures 4.9 to 4.11 show the streamline and isotherms for $\delta=0.1$ while the Grashof number is $1 \times 10^{3}, 5 \times 10^{4}$ and $5 \times 10^{6}$.
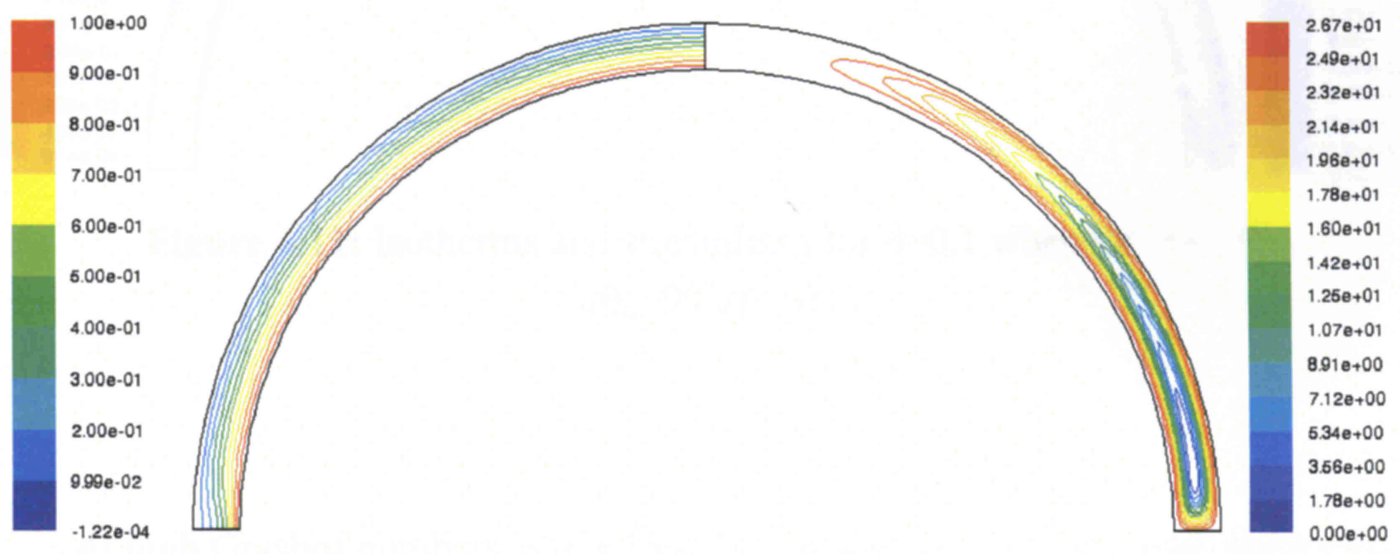

Figure 4.9: Isotherms and streamlines for $\delta=0.1$ when $\mathrm{Gr}=1 \times 10^{3}$

$$
\left(\theta_{0}=90^{\circ}, \varepsilon=0\right)
$$

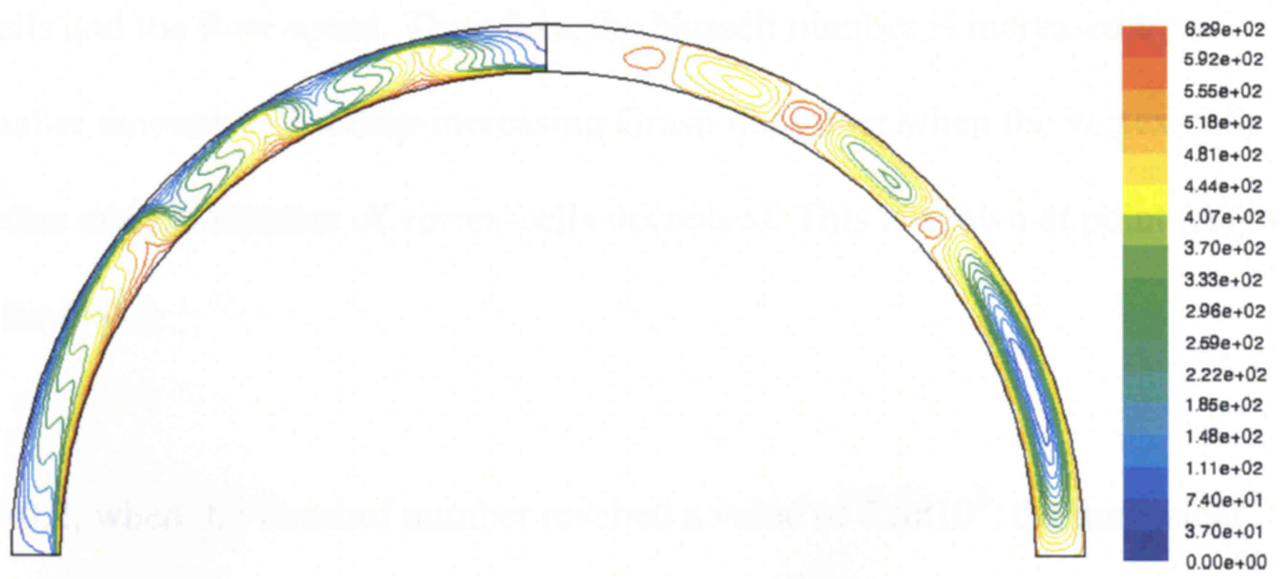

Figure 4.10: Isotherms and streamlines for $\delta=0.1$ when $\mathrm{Gr}=5 \times 10^{4}$

$$
\left(\theta_{0}=90^{\circ}, \varepsilon=0\right)
$$




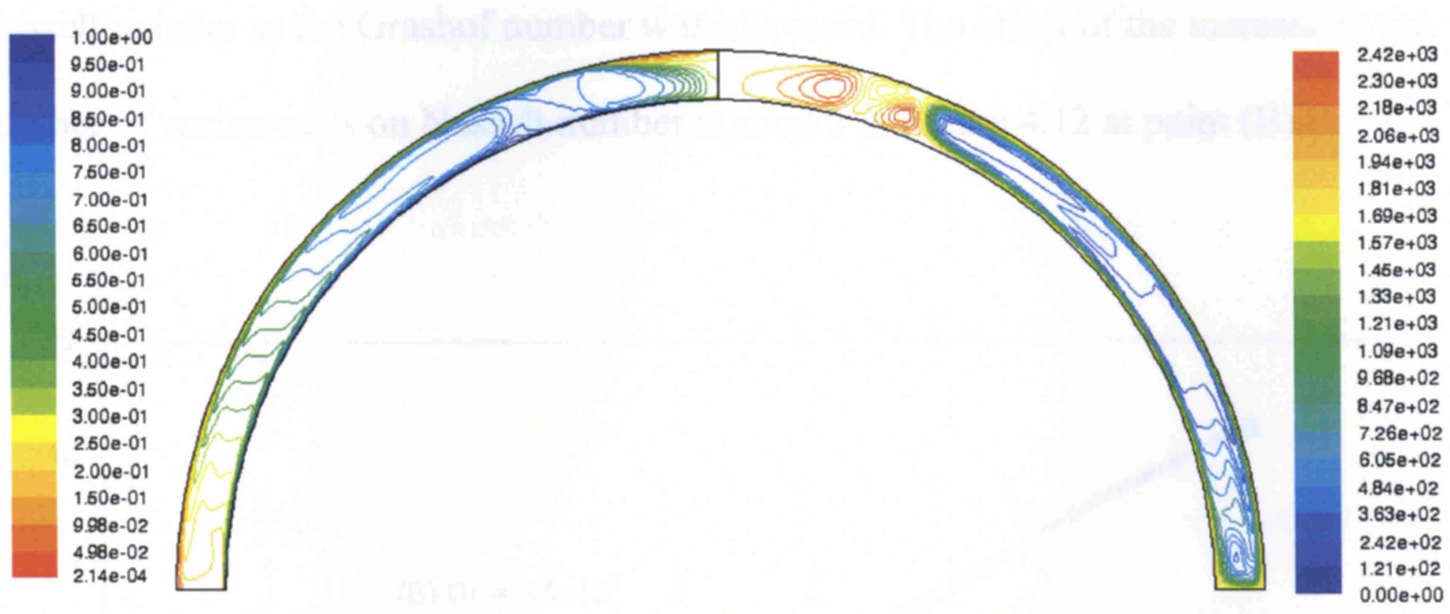

Figure 4.11: Isotherms and streamlines for $\delta=0.1$ when $\mathrm{Gr}=5 \times 10^{6}$

$$
\left(\theta_{0}=90^{\circ}, \varepsilon=0\right)
$$

At high Grashof numbers $\left(G r>5 \times 10^{4}\right)$, the numerical results may reach steady state or unsteady flow conditions with multi-vortex-cells. As the Grashof number increased, the vortex-cells on top of the main vortex-cell tended to merge with the main vortex-cell at the bottom. The heat transfer within the cavity is related to the number of the vortex-cells and the flow speed. Therefore, the Nusselt number is increased by a relatively smaller amount with a step-increasing Grashof number when the vortex-cells merged together and the number of vortex-cells decreased. This is shown at point (A) in Figure 4.12 for $\delta=0.2$.

At $\delta=0.2$, when the Grashof number reached a value of $7.5 \times 10^{5}$, the numerical results converged to the unsteady state again, and new vortex-cells formed in the top region of the cavity. The newly formed vortex-cells caused an additional increase in the 
Nusselt number as the Grashof number was increased. The effect of the increase in the number of vortex-cells on Nusselt number is shown in Figure 4.12 at point (B).

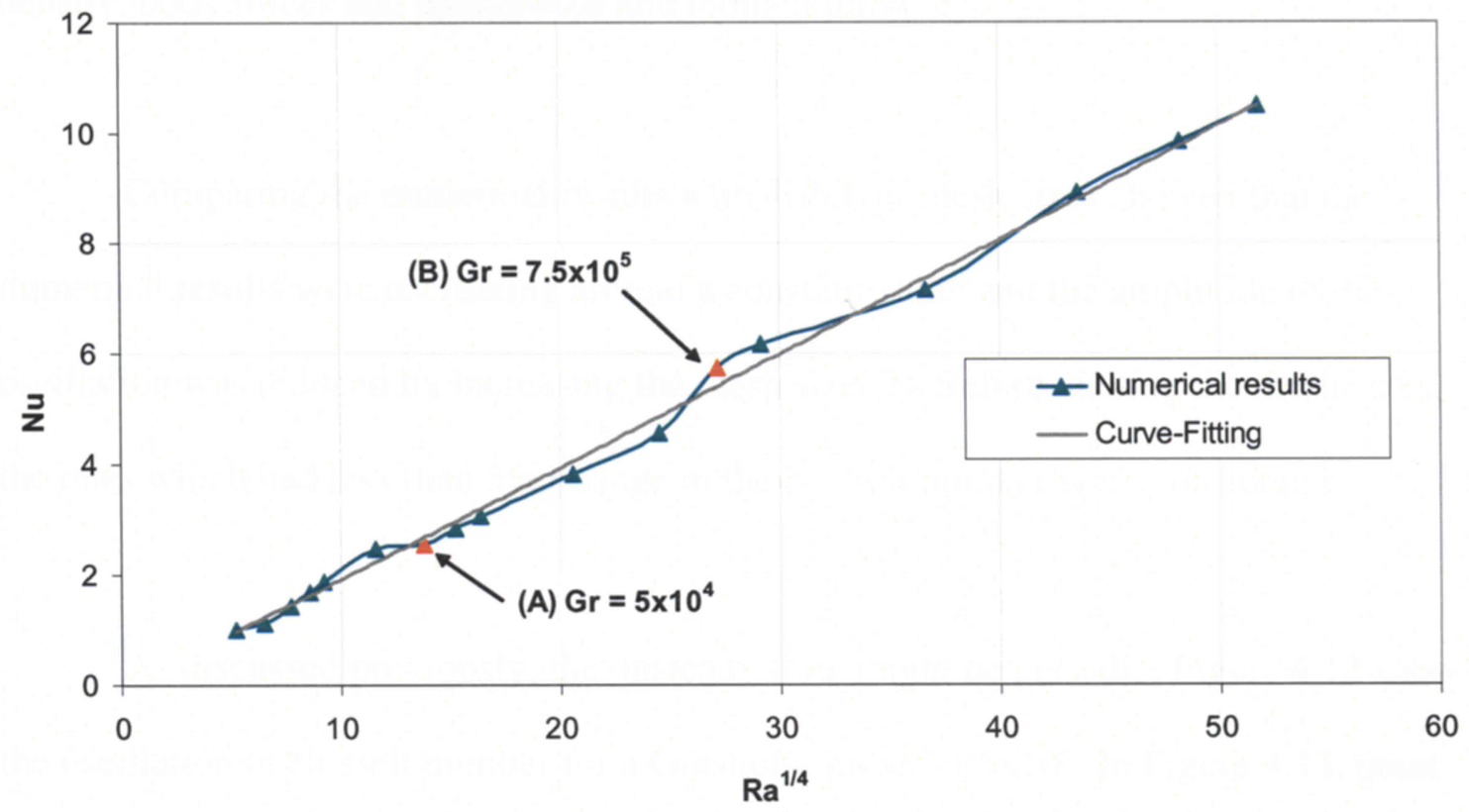

Figure 4.12: Changes in the Nusselt versus Rayleigh number to the power of $(1 / 4)$ $\left(\theta_{0}=90^{\circ}, \varepsilon=0\right.$ and $\left.\delta=0.2\right)$

For the smallest gap-to-radius ratio $(\delta=0.01)$, all simulations were run in the unsteady mode, because oscillations in heat transfer from the exterior surface were observed at most Grashof numbers. Since the radius is relatively big compared to the gap spacing when $\delta=0.01$, the number of control-volume-cells in the meshed geometry was increased significantly. Consequently, increasing the number of control-volume-cells caused an increase in the number of numerical calculations per iteration. Therefore, the numerical process was slowed down significantly. The numerical results showed that the 
changes in the Nusselt number were periodic and the time period was relatively large. As a result, running the simulation for each mesh size took more than a week. The under-relaxation factor's values were reduced from the original value (pressure $=0.25$, density, body forces and energy $=0.8$ and momentum $=0.6$ ).

Comparing the numerical results with different mesh sizes showed that the numerical results were oscillating around a constant value and the amplitude of the oscillation was reduced by increasing the mesh size. Therefore, among the mesh sizes, the ones which had less than 5\% change in the Nusselt number were considered.

As discussed previously, the unsteady flow might be periodic. Figure 4.13 shows the oscillation in Nusselt number for a Grashof number of $5 \times 10^{5}$. In Figure 4.13, point (A) is the point that the time step was changed from the value of 1.0 to the value of 0.1 in the numerical analysis. In the numerical procedure different time step sizes were considered to make sure that the results were independent of time step size. Figure 4.13 shows a comparison between Nusselt number for time step sizes equal to 1 and 0.1 . It is concluded that changing the time step size has no effect on the average Nusselt number if the Nusselt number is calculated by averaging within a time period much bigger than the oscillation period. 


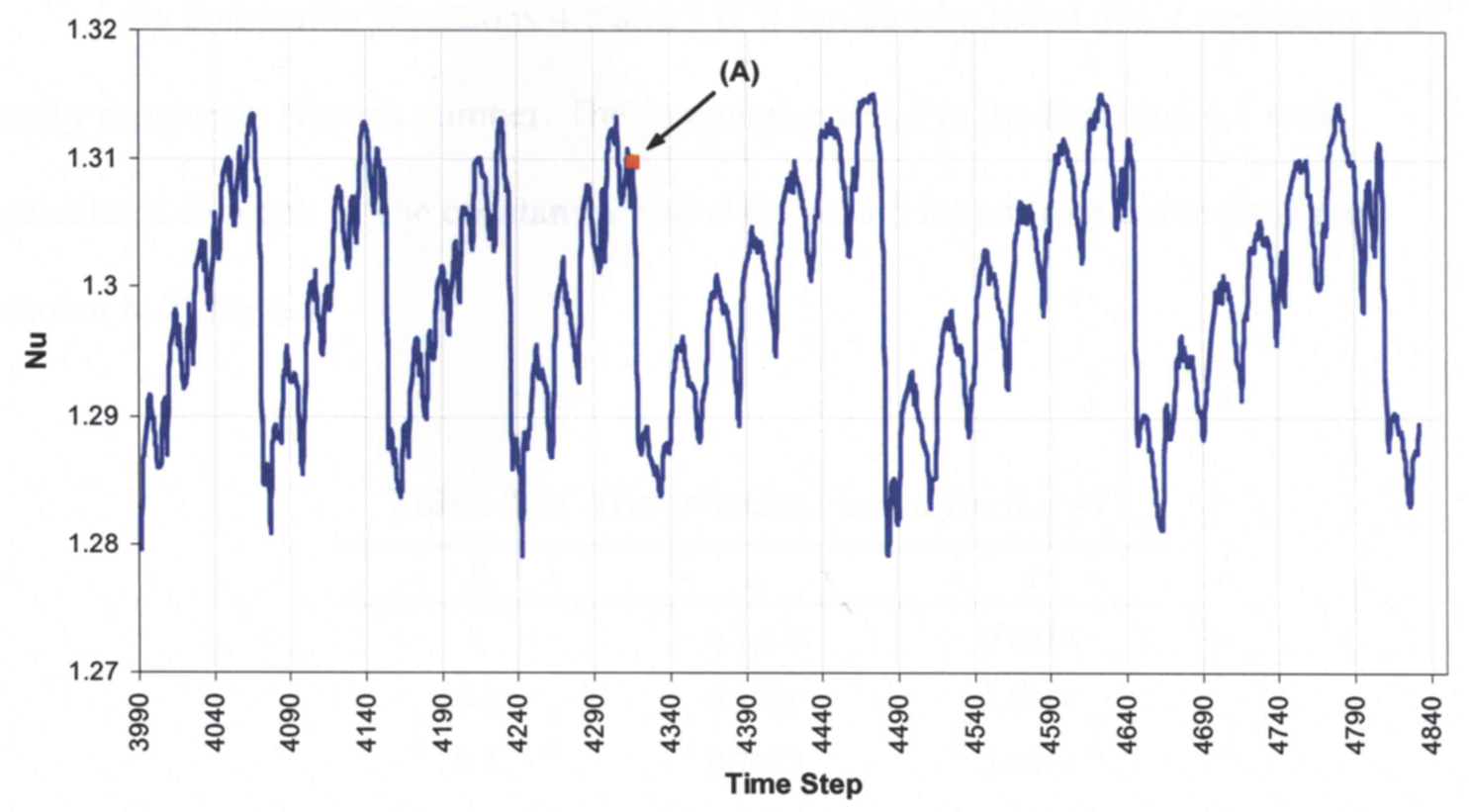

Figure 4.13: Oscillation in Nusselt number with the time step $=1$ and 0.1

$$
\left(\mathrm{Gr}=5 \times 10^{5}, \theta_{0}=90^{\circ}, \varepsilon=0 \text { and } \delta=0.01\right)
$$

\subsubsection{Correlations for Heat Transfer}

The average Nusselt number calculated from the present model for a truncation angle of $90^{\circ}$ and for different values of $\delta$, is presented in Appendix-A, Table A.1. The main objective of this study is to find a simplified correlation similar to Equation 3.6. Therefore, the power Equation 4.1 was curve-fitted to the numerical results for each value of $\delta$.

$$
y=c x^{d}
$$


By comparing Equations 4.1 and 3.6, it can be concluded that $x$ represents $\mathrm{Ra}^{*^{1 / 4}}$ and $y$ represents Nusselt number. The constants $c$ and $d$ in the Equation 4.1 were calculated for each $\delta$. The constants $c$ and $d$ for each $\delta$ found from curve-fitting are shown in Table 4.1.

Table 4.1: The constant $c$ and $d$ for $\theta_{0}=90^{\circ}$

\begin{tabular}{ccc}
\hline $\boldsymbol{\delta}$ & $\boldsymbol{c}$ & $\boldsymbol{d}$ \\
\hline 1 & 0.7801 & 0.9914 \\
0.8 & 0.7721 & 1.0068 \\
0.5 & 0.7622 & 1.0258 \\
0.4 & 0.7610 & 1.0293 \\
0.3 & 0.7402 & 1.0513 \\
0.2 & 0.8164 & 1.0339 \\
0.1 & 0.8994 & 1.0097 \\
0.01 & 0.8430 & 0.9869 \\
\hline
\end{tabular}

The Nusselt number calculated from the present model are compared with the Nusselt number calculated from curve-fitting Equation 4.1. Figure 4.14 shows a sample of curve-fitting of Equation 4.1 to the numerical results when $\delta=0.4$. Figure 4.14 shows the best-fit curve of the numerical results with $\pm 4 \%$ error bars. 


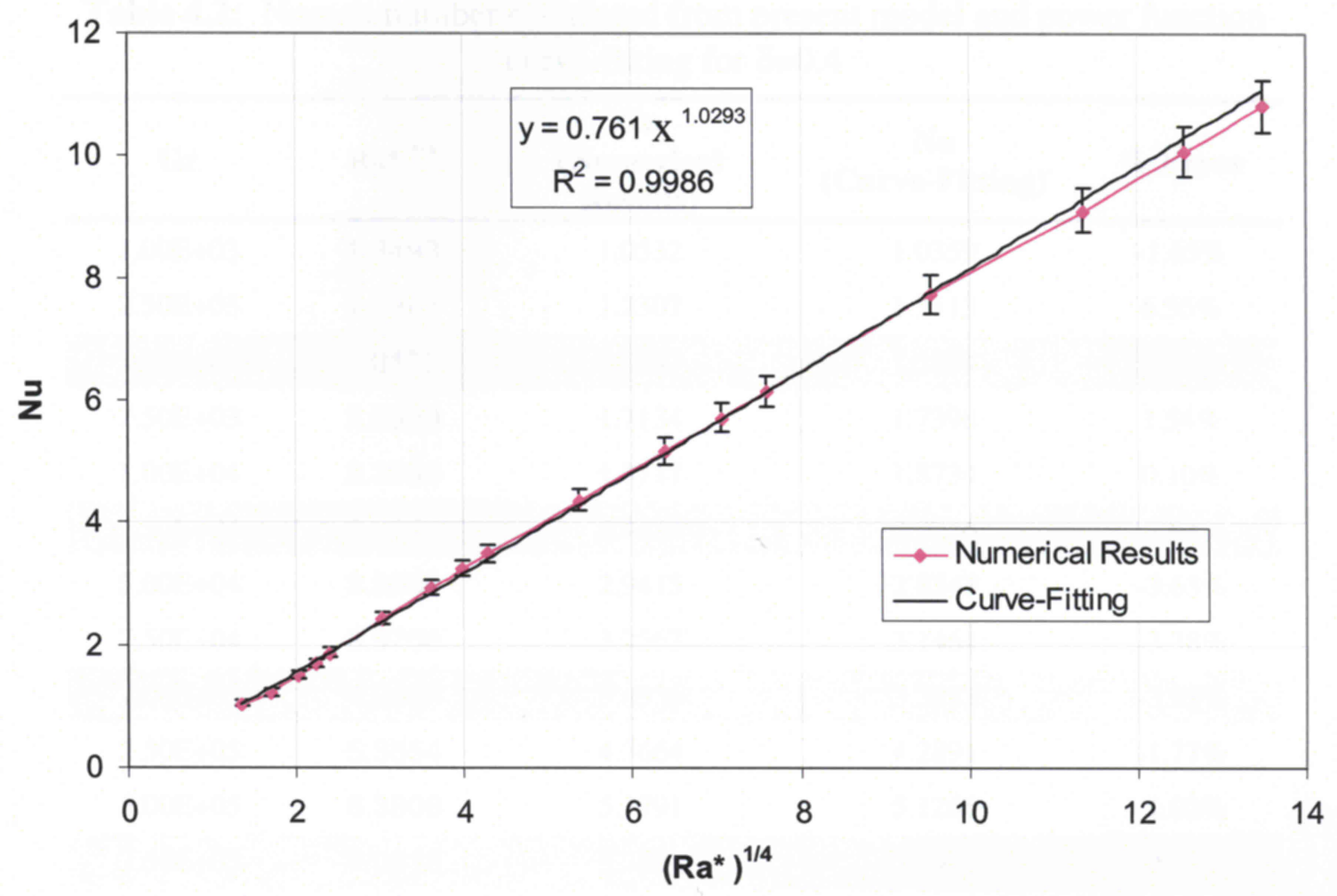

Figure 4.14: Numerically predicted Nusselt number (with $\pm 4 \%$ error bars) and power function curve-fitted to the numerical results when $\delta=0.4$.

Table 4.2 compares the numerical results and the results from Equation 4.1 for $\delta=0.4(c=0.761$ and $d=1.0293)$. From the results in Table 4.2 , it can be concluded the power function that was curve-fitted to the numerical results predicts the Nusselt number with less than $4 \%$ error, except at a Grashof number of $2.5 \times 10^{3}$, where the percentage error is about $7 \%$. 
Table 4.2: Nusselt number calculated from present model and power function curve-fitting for $\delta=0.4$

\begin{tabular}{ccccc}
\hline $\mathbf{G r}$ & $\mathbf{R a}^{* 1 / 4}$ & $\begin{array}{c}\text { Nu } \\
\text { (Numerical } \\
\text { Results) }\end{array}$ & $\begin{array}{c}\text { Nu } \\
\text { (Curve-Fitting) }\end{array}$ & \% Error \\
\hline $1.00 \mathrm{E}+03$ & 1.3493 & 1.0532 & 1.0359 & $-1.65 \%$ \\
$2.50 \mathrm{E}+03$ & 1.6967 & 1.2307 & 1.3113 & $6.55 \%$ \\
\hline $5.00 \mathrm{E}+03$ & 2.0177 & 1.5087 & 1.5674 & $3.89 \%$ \\
$7.50 \mathrm{E}+03$ & 2.2330 & 1.7134 & 1.7398 & $1.54 \%$ \\
$1.00 \mathrm{E}+04$ & 2.3995 & 1.8717 & 1.8734 & $0.10 \%$ \\
\hline $2.50 \mathrm{E}+04$ & 3.0172 & 2.4434 & 2.3716 & $-2.94 \%$ \\
\hline $5.00 \mathrm{E}+04$ & 3.5881 & 2.9415 & 2.8347 & $-3.63 \%$ \\
$7.50 \mathrm{E}+04$ & 3.9708 & 3.2563 & 3.1464 & $-3.38 \%$ \\
\hline $1.00 \mathrm{E}+05$ & 4.2669 & 3.4936 & 3.3882 & $-3.02 \%$ \\
\hline $2.50 \mathrm{E}+05$ & 5.3654 & 4.3664 & 4.2891 & $-1.77 \%$ \\
\hline $5.00 \mathrm{E}+05$ & 6.3806 & 5.1791 & 5.1266 & $-1.02 \%$ \\
\hline $7.50 \mathrm{E}+05$ & 7.0613 & 5.7268 & 5.6903 & $-0.64 \%$ \\
\hline $1.00 \mathrm{E}+06$ & 7.5878 & 6.1510 & 6.1276 & $-0.38 \%$ \\
\hline $2.50 \mathrm{E}+06$ & 9.5412 & 7.7319 & 7.7569 & $0.32 \%$ \\
\hline $5.00 \mathrm{E}+06$ & 11.3464 & 9.0960 & 9.2715 & $1.93 \%$ \\
\hline $7.50 \mathrm{E}+06$ & 12.5569 & 10.0676 & 10.2911 & $2.22 \%$ \\
\hline $1.00 \mathrm{E}+07$ & 13.4933 & 10.8183 & 11.0819 & $2.44 \%$ \\
\hline & & & & \\
\hline
\end{tabular}

The constants $c$ and $d$ are a function of $\delta$. It would be useful to define a general correlation for the constants $c$ and $d$ as a function of $\delta$. TableCurve version 1.10 software was used to find the best correlation that could be fitted to the constants $c$ and $d$. TableCurve software uses a least squares method to find the constants of different functions and fit them to the input values. Equations 4.2 and 4.3 were found from curve-fitting using TableCurve. 


$$
\begin{gathered}
c=\frac{0.77923+0.88266 \ln (\delta)+0.27049(\ln (\delta))^{2}}{1+1.10818 \ln (\delta)+0.33058(\ln (\delta))^{2}} \\
d=\frac{0.99443+1.12137 \ln (\delta)+0.50497(\ln (\delta))^{2}}{1+1.15263 \ln (\delta)+0.51559(\ln (\delta))^{2}}
\end{gathered}
$$

Figures 4.15 and 4.16 show the Equations 4.2 and 4.3 that curve-fitted to the value of constant $c$ and $d$ respectively.

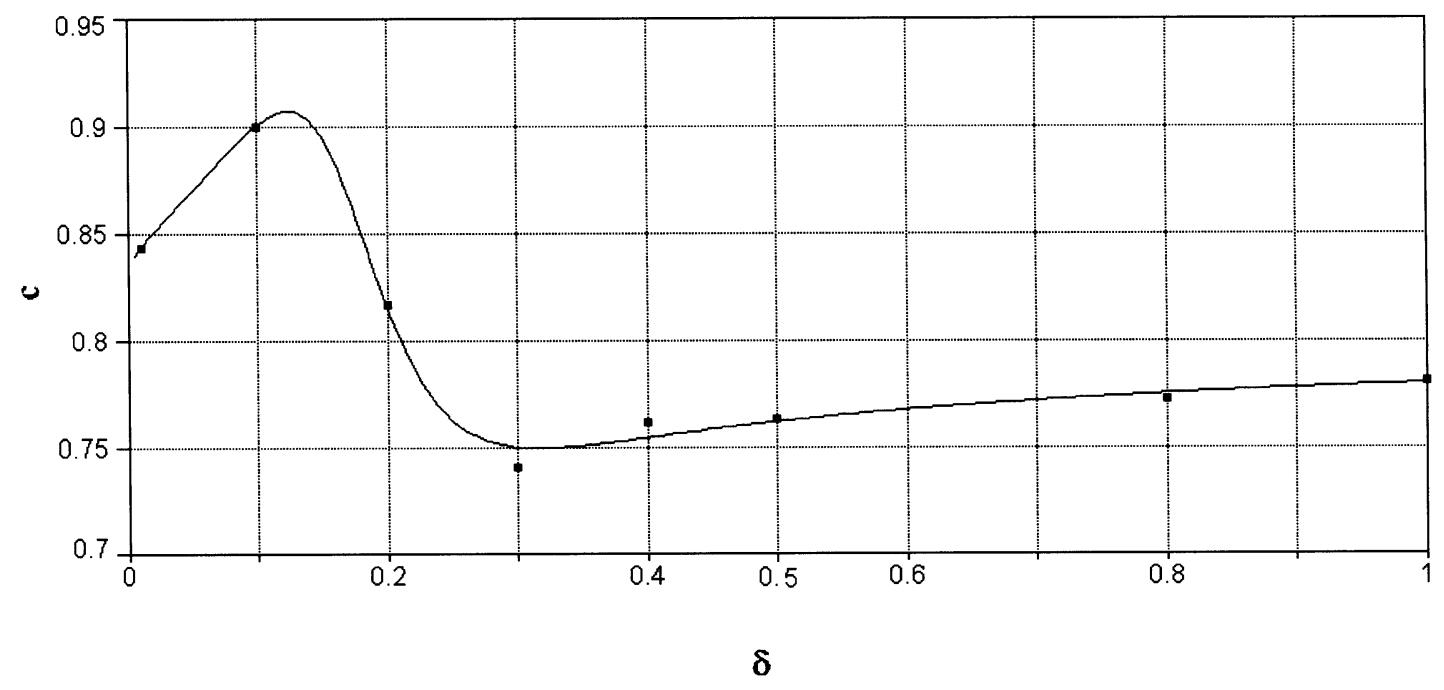

Figure 4.15: The curve-fitted (Equation 4.2) to the constant $c$ 


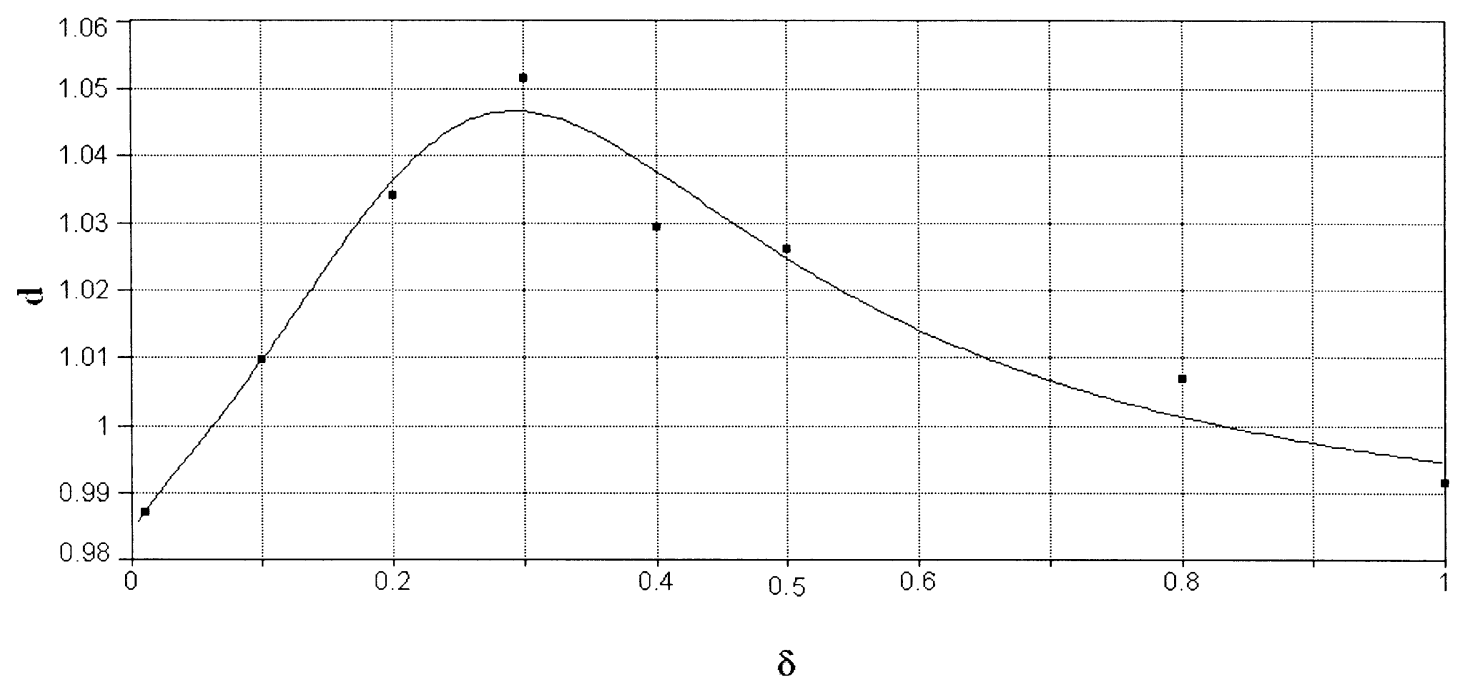

Figure 4.16: The curve-fitted (Equation 4.3) to the constant $d$

Table 4.3 compares the values of $c$ and $d$, which were calculated from curve-fitting Equation 4.1 to the numerical results (Table 4.1) and Equations 4.2 and 4.3. From Table 4.3, it can be concluded that Equations 4.2 and 4.3 could predict the values of $c$ and $d$ with less than $1.5 \%$ error.

Table 4.3: constant $c$ and $d$ for $\theta_{0}=90^{\circ}$

\begin{tabular}{ccccccc}
\hline $\boldsymbol{\delta}$ & $\boldsymbol{c}$ & $\begin{array}{c}\boldsymbol{c} \\
(\mathbf{E q . 4 . 2})\end{array}$ & \% Error & $\boldsymbol{d}$ & $\begin{array}{c}\boldsymbol{d} \\
(\mathbf{E q . 4 . 3})\end{array}$ & \% Error \\
\hline 1 & 0.7801 & 0.7792 & $0.11 \%$ & 0.9914 & 0.9944 & $0.31 \%$ \\
0.8 & 0.7721 & 0.7745 & $0.31 \%$ & 1.0068 & 1.0011 & $0.56 \%$ \\
0.5 & 0.7622 & 0.7611 & $0.14 \%$ & 1.0258 & 1.0245 & $0.13 \%$ \\
0.4 & 0.7610 & 0.7537 & $0.96 \%$ & 1.0293 & 1.0376 & $0.80 \%$ \\
0.3 & 0.7402 & 0.7493 & $1.23 \%$ & 1.0513 & 1.0464 & $0.47 \%$ \\
0.2 & 0.8164 & 0.8151 & $0.16 \%$ & 1.0339 & 1.0359 & $0.19 \%$ \\
0.1 & 0.8994 & 0.9002 & $0.09 \%$ & 1.0097 & 1.0094 & $0.03 \%$ \\
0.01 & 0.8430 & 0.8430 & $0.00 \%$ & 0.9869 & 0.9869 & $0.00 \%$ \\
\hline
\end{tabular}


Therefore, the Nusselt number which was calculated from the present model can be estimated with the following equation:

$$
N u=\max \left\{1, c\left(R a^{*}\right)^{d / 4}\right\}
$$

where the constants $c$ and $d$ can be calculated from Equations 4.2 and 4.3 respectively.

The average percentage difference between the Nusselt number which is calculated from present model and Equation 4.4 for each $\delta$ is less than 5\%. The average percentage error is defined as:

$$
\text { Averaged \% Error }=\sum_{1}^{n} \frac{\mid \% \text { Error } \mid}{n}
$$

Table 4.4 shows a comparison between the Nusselt number found from the numerical model and the Nusselt number calculated from Equation 4.4. Table 4.4 shows that the maximum percentage error for $\delta=0.4$ is about $6 \%$ at the Grashof number of $2.5 \times 10^{3}$. Maximum percentage error for truncation angle of $90^{\circ}$, considering all gap-to-radius ratios, is less then $12 \%$ which is found for $\delta=0.1$ at $\mathrm{Gr}=5 \times 10^{3}$. 
Table 4.4: The Nusselt number comparison between the present numerical model and Equation 4.4 for $\theta_{0}=90^{\circ} \delta=0.4$.

\begin{tabular}{ccccc}
\hline $\mathbf{G r}$ & $\mathbf{R a}^{* 1 / 4}$ & $\begin{array}{c}\text { Nu } \\
\text { (Numerical } \\
\text { Results) }\end{array}$ & $\begin{array}{c}\text { Nu } \\
\text { (Equation. 4.4 })\end{array}$ & \% Error \\
\hline $1.00 \mathrm{E}+03$ & 1.3493 & 1.0532 & 1.0284 & $2.36 \%$ \\
$2.50 \mathrm{E}+03$ & 1.6967 & 1.2307 & 1.3044 & $5.98 \%$ \\
\hline $5.00 \mathrm{E}+03$ & 2.0177 & 1.5087 & 1.5613 & $3.49 \%$ \\
$7.50 \mathrm{E}+03$ & 2.2330 & 1.7134 & 1.7345 & $1.23 \%$ \\
$1.00 \mathrm{E}+04$ & 2.3995 & 1.8717 & 1.8688 & $0.15 \%$ \\
\hline $2.50 \mathrm{E}+04$ & 3.0172 & 2.4434 & 2.3703 & $2.99 \%$ \\
$5.00 \mathrm{E}+04$ & 3.5881 & 2.9415 & 2.8371 & $-.55 \%$ \\
$7.50 \mathrm{E}+04$ & 3.9708 & 3.2563 & 3.1518 & $3.21 \%$ \\
$1.00 \mathrm{E}+05$ & 4.2669 & 3.4936 & 3.3960 & $2.79 \%$ \\
\hline $2.50 \mathrm{E}+05$ & 5.3654 & 4.3664 & 4.3071 & $1.36 \%$ \\
\hline $5.00 \mathrm{E}+05$ & 6.3806 & 5.1791 & 5.1555 & $0.46 \%$ \\
\hline $7.50 \mathrm{E}+05$ & 7.0613 & 5.7268 & 5.7273 & $0.01 \%$ \\
\hline $1.00 \mathrm{E}+06$ & 7.5878 & 6.1510 & 6.1710 & $0.33 \%$ \\
\hline $2.50 \mathrm{E}+06$ & 9.5412 & 7.7319 & 7.8267 & $1.23 \%$ \\
\hline $5.00 \mathrm{E}+06$ & 11.3464 & 9.0960 & 9.3684 & $2.99 \%$ \\
\hline $7.50 \mathrm{E}+06$ & 12.5569 & 10.0676 & 10.4074 & $3.37 \%$ \\
$1.00 \mathrm{E}+07$ & 13.4933 & 10.8183 & 11.2137 & $3.65 \%$ \\
\hline
\end{tabular}

Table 4.5 shows the average percentage error for $\theta_{0}=90^{\circ}$ calculated from Equation 4.5 for different values of $\delta$. Table 4.5 shows that the average percentage error for a truncation angle of $90^{\circ}$ which is less than $5 \%$. 
Table 4.5: The average percentage error in Nusselt number calculated from Equation 4.4 and present model for $\theta_{0}=90^{\circ}$.

\begin{tabular}{cc}
\hline $\boldsymbol{\delta}$ & $\begin{array}{c}\text { Average } \\
\text { \% Error }\end{array}$ \\
\hline 1 & $1.73 \%$ \\
0.8 & $1.63 \%$ \\
0.5 & $1.98 \%$ \\
0.4 & $2.30 \%$ \\
0.3 & $2.06 \%$ \\
0.2 & $4.56 \%$ \\
0.1 & $4.39 \%$ \\
0.01 & $1.30 \%$ \\
\hline
\end{tabular}

Figure 4.17 shows all numerical results for the Nusselt number, as well as calculated values based on Equation 4.4 for a truncation angle of $90^{\circ}$.

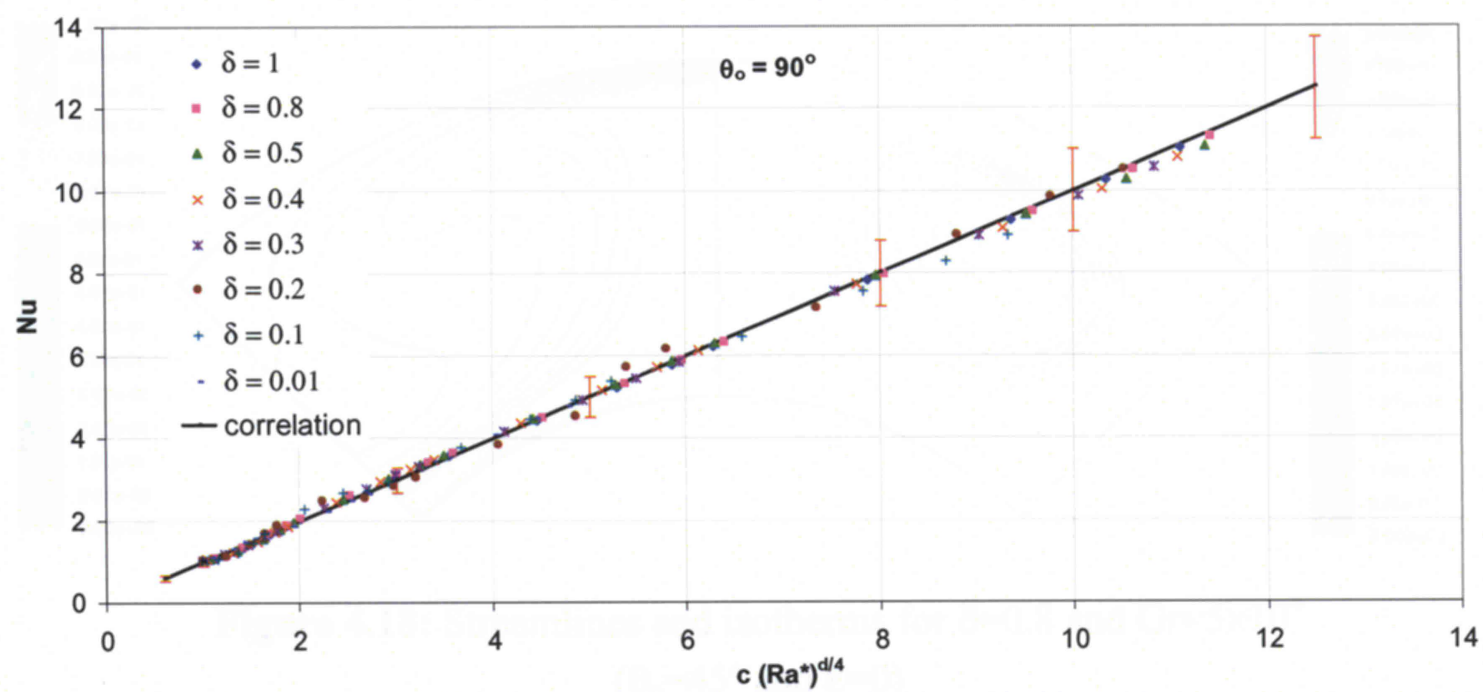

Figure 4.17: Nusselt number correlation with $\pm 10 \%$ error bar and numerical results for $\theta_{0}=90^{\circ}$. 


\subsubsection{Concentric Cavities with Truncation Angle of $\left(\theta_{0}=\mathbf{4 5}^{\circ}\right)$}

Numerical results for domed cavities with a truncation angle of $45^{\circ}$ showed that, for large gap-to-radius ratios $(0.5 \leq \delta \leq 1)$, the flow may reach the steady state condition with one vortex-cell for the studied range of Grashof numbers. For small Grashof numbers, a flow type similar to that for a truncation angle of $90^{\circ}$ was observed. Figures 4.18 and 4.19 show the isotherms and streamlines for $\delta=0.8$ at Grashof numbers of $5 \times 10^{4}$ and $5 \times 10^{6}$, respectively. For very large Grashof numbers, small unstable vortex-cells appeared inside the main vortex-cell. The unstable vortex-cells caused small oscillations in the Nusselt number at high Grashof numbers.

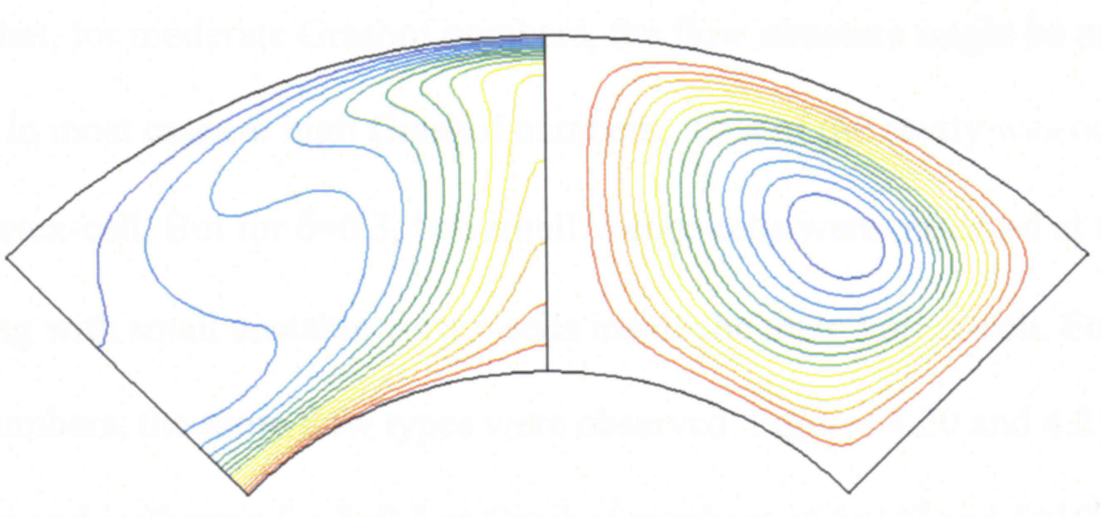

Figure 4.18: Streamlines and isotherms for $\delta=0.8$ and $\mathrm{Gr}=5 \times 10^{4}$

$$
\left(\theta_{0}=45^{\circ} \text { and } \varepsilon=0\right)
$$



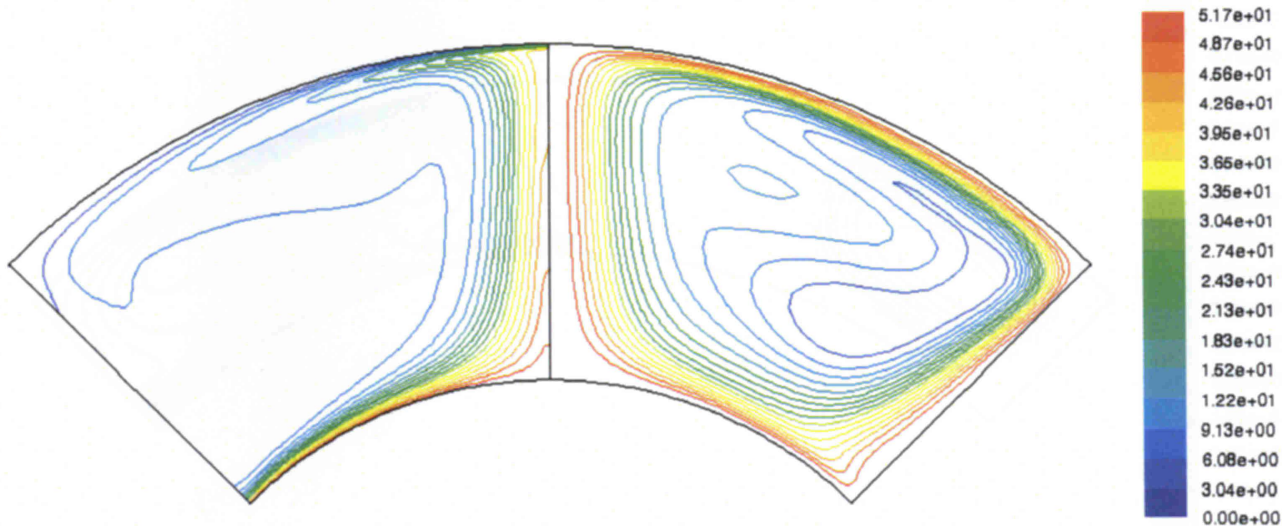

Figure 4.19: Streamlines and isotherms for $\delta=0.8$ and $\mathrm{Gr}=5 \times 10^{6}$

$$
\left(\theta_{\mathrm{o}}=45^{\circ} \text { and } \varepsilon=0\right)
$$

Numerical results for moderate gap-to-radius ratios $(0.5<\delta<0.2)$ showed that the flow may reach the steady state condition. For these geometries, the fluid flow was comprised of a single vortex-cell for relatively small Grashof numbers. However, it was observed that, for moderate Grashof numbers, the flow structure might be multi-cellular in nature. In most cases at high Grashof numbers, most of the cavity was occupied with one big vortex-cell. But for $\delta=0.3$, two small vortex-cells were observed at the top of the cavity along with small unstable vortex-cells inside the main vortex-cell. For small Grashof numbers, the same flow types were observed. Figures 4.20 and 4.21 show the streamlines and isotherms for $\delta=0.3$ at Grashof numbers of $5 \times 10^{4}$ and $5 \times 10^{6}$. 

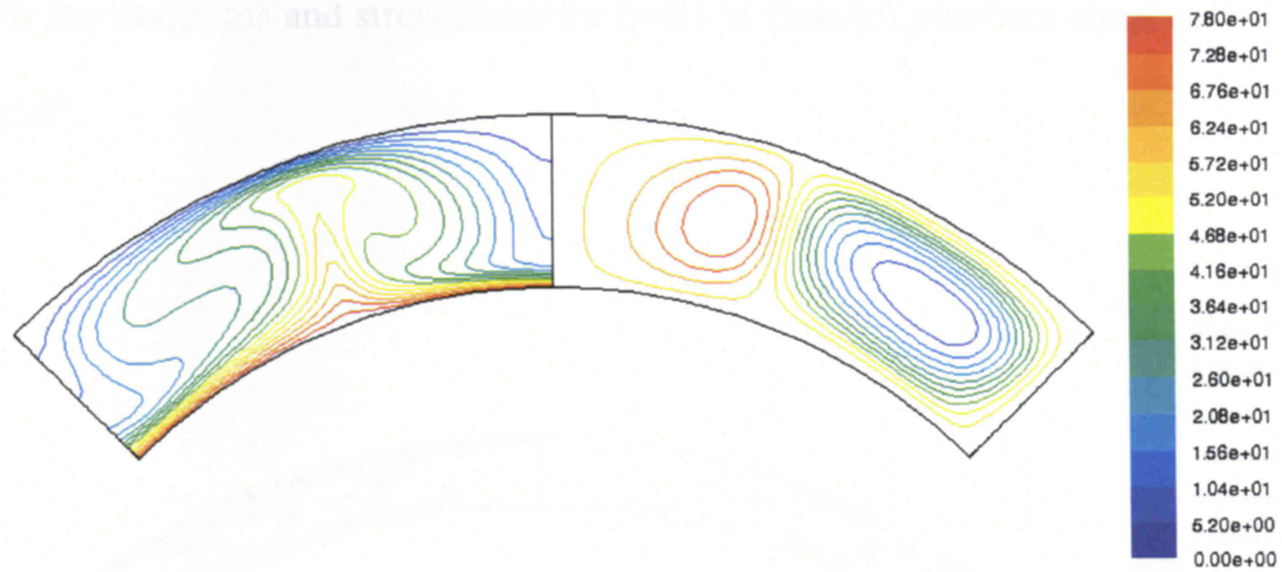

Figure 4.20: Streamlines and isotherms for $\delta=0.3$ and $\mathrm{Gr}=5 \times 10^{4}$ $\left(\theta_{0}=45^{\circ}\right.$ and $\left.\varepsilon=0\right)$
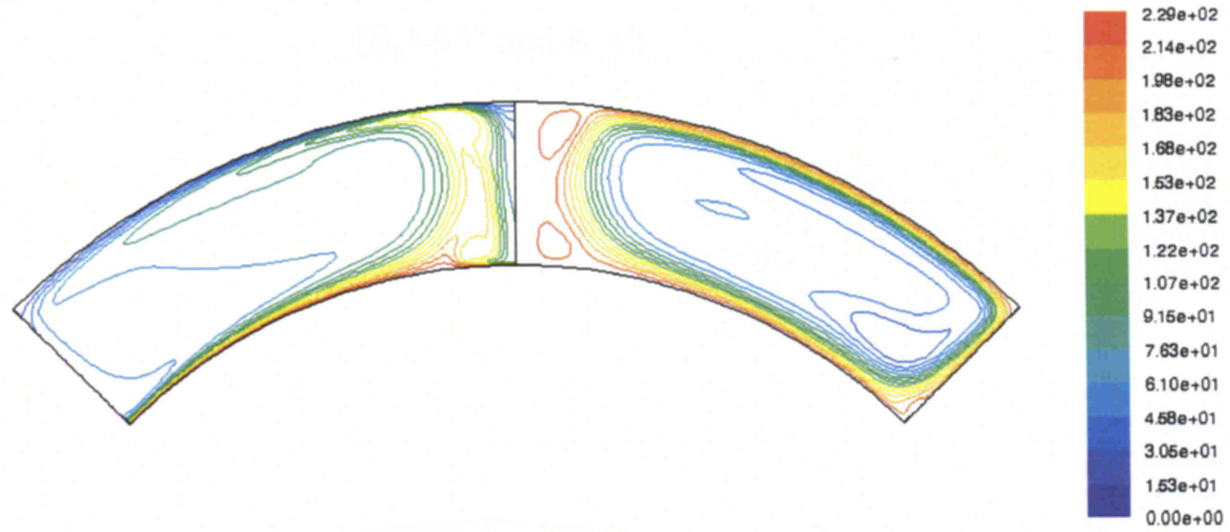

Figure 4.21: Streamlines and isotherms for $\delta=0.3$ and $\mathrm{Gr}=5 \times 10^{6}$

$$
\left(\theta_{0}=45^{\circ} \text { and } \varepsilon=0\right)
$$

The numerical cases for small gap-to-radius cavities $(\delta \leq 0.2)$ converged very slowly. A single-vortex-cell was observed for small Grashof numbers. The fluid flow was unsteady with multi-vortex-cells for moderate and high Grashof numbers. Figures 4.22 
and 4.23 show the isotherms and streamlines for $\delta=0.1$ at Grashof numbers equal to $5 \times 10^{4}$ and $5 \times 10^{6}$.
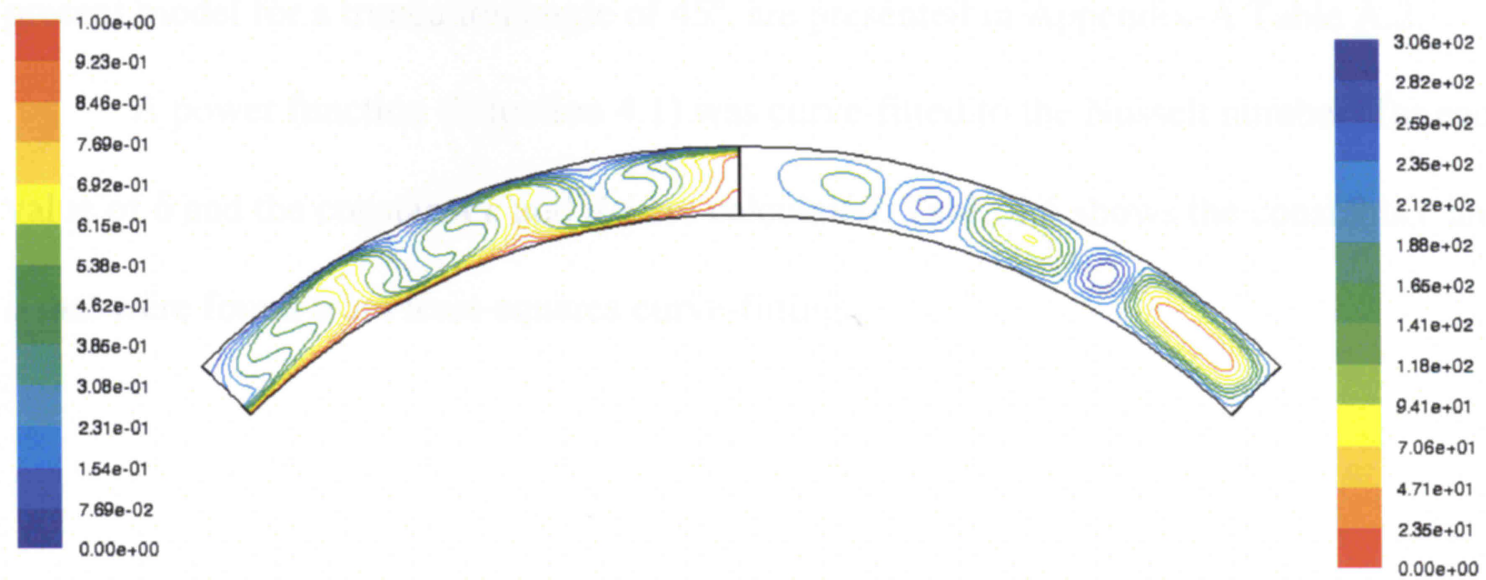

Figure 4.22: Streamlines and isotherms for $\delta=0.1$ and $\mathrm{Gr}=5 \times 10^{4}$ $\left(\theta_{\mathrm{o}}=45^{\circ}\right.$ and $\left.\varepsilon=0\right)$

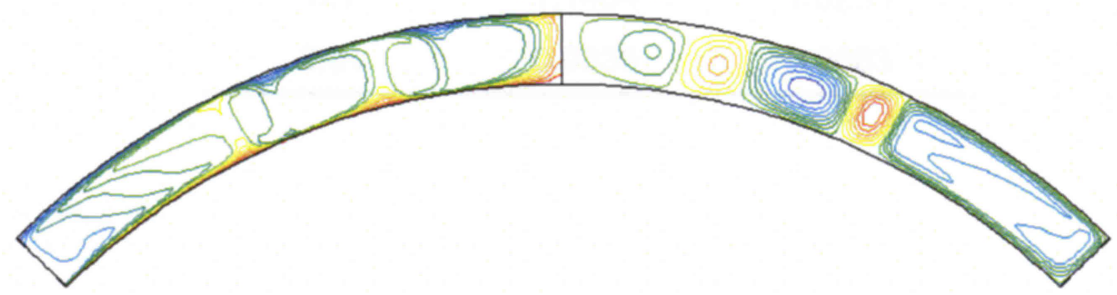

Figure 4.23: Streamlines and isotherms for $\delta=0.1$ and $\mathrm{Gr}=5 \times 10^{6}$

$$
\left(\theta_{0}=45^{\circ} \text { and } \varepsilon=0\right)
$$




\subsubsection{Correlations for Heat Transfer}

Similar to the results for a truncation angle of $90^{\circ}$, periodic behavior in the Nusselt number was observed. The Nusselt number data, which were calculated from the present model for a truncation angle of $45^{\circ}$, are presented in Appendix-A Table A.2.

A power function (Equation 4.1) was curve-fitted to the Nusselt numbers for each value of $\delta$ and the constants $c$ and $d$ were calculated. Table 4.6 shows the constants $c$ and $d$ that were found from least-squares curve-fitting.

Table 4.6: The constant $c$ and $d$ for $\theta_{0}=45^{\circ}$

\begin{tabular}{ccc}
\hline $\boldsymbol{\delta}$ & $\boldsymbol{c}$ & $\boldsymbol{d}$ \\
\hline 1 & 0.6992 & 1.0435 \\
0.8 & 0.7436 & 1.0373 \\
0.5 & 0.8337 & 1.0233 \\
0.4 & 0.8229 & 1.0447 \\
0.3 & 0.9049 & 1.0239 \\
0.2 & 2.1270 & 0.9809 \\
0.1 & 1.1404 & 1.0233 \\
0.01 & 0.9335 & 0.9683 \\
\hline
\end{tabular}

Figure 4.24 shows the Nusselt number calculated from the present model with $\pm 4 \%$ error bars for $\delta=0.5$ and the power function curve-fitted to it. 




Figure 4.24: Nusselt number from numerical result ( $\pm 4 \%$ error bar) and power function fitted to the numerical results for $\delta=0.5$

Table 4.7 compares the Nusselt number calculated from the present model and the Nusselt number found from Equation 4.1 for $\delta=0.5$. 
Table 4.7: Nusselt number calculated from present model and power function curve-fitting in $\delta=0.5$

\begin{tabular}{|c|c|c|c|c|}
\hline $\mathbf{G r}$ & $\mathbf{R a} *^{1 / 4}$ & $\begin{array}{c}\mathrm{Nu} \\
\text { (Present Model) }\end{array}$ & $\begin{array}{c}\mathrm{Nu} \\
\text { (Equation 4.1) }\end{array}$ & \% Error \\
\hline $1.00 \mathrm{E}+03$ & 1.3930 & 1.0528 & 1.1000 & $4.49 \%$ \\
\hline $2.50 \mathrm{E}+03$ & 1.7516 & 1.3405 & 1.4795 & $10.37 \%$ \\
\hline $5.00 \mathrm{E}+03$ & 2.0830 & 1.7396 & 1.7665 & $1.55 \%$ \\
\hline $7.50 \mathrm{E}+03$ & 2.3052 & 1.9885 & 1.9596 & $-1.46 \%$ \\
\hline $1.00 \mathrm{E}+04$ & 2.4771 & 2.1684 & 2.1093 & $-2.73 \%$ \\
\hline $2.50 \mathrm{E}+04$ & 3.1148 & 2.7785 & 2.6664 & $-4.03 \%$ \\
\hline $5.00 \mathrm{E}+04$ & 3.7041 & 3.3015 & 3.1838 & $-3.56 \%$ \\
\hline $7.50 \mathrm{E}+04$ & 4.0993 & 3.6403 & 3.5318 & $-2.98 \%$ \\
\hline $1.00 \mathrm{E}+05$ & 4.4049 & 3.8981 & 3.8015 & $-2.48 \%$ \\
\hline $2.50 \mathrm{E}+05$ & 5.5389 & 4.8400 & 4.8057 & $-0.71 \%$ \\
\hline $5.00 \mathrm{E}+05$ & 6.5869 & 5.7168 & 5.7381 & $0.37 \%$ \\
\hline $7.50 \mathrm{E}+05$ & 7.2896 & 6.3106 & 6.3653 & $0.87 \%$ \\
\hline $1.00 \mathrm{E}+06$ & 7.8332 & 6.7758 & 6.8514 & $1.12 \%$ \\
\hline $2.50 \mathrm{E}+06$ & 9.8498 & 8.5590 & 8.6613 & $1.20 \%$ \\
\hline $5.00 \mathrm{E}+06$ & 11.7134 & 10.2328 & 10.3417 & $1.06 \%$ \\
\hline $7.50 \mathrm{E}+06$ & 12.9630 & 11.3537 & 11.4721 & $1.04 \%$ \\
\hline $1.00 \mathrm{E}+07$ & 13.9297 & 12.2158 & 12.3482 & $1.08 \%$ \\
\hline
\end{tabular}

Equations 4.6 and 4.7 can be found by following the same steps that were taken for a truncation angle of $90^{\circ}$ using TableCurve software.

$$
c=\left\{\begin{array}{l}
\frac{1}{1.15362-49.65900 \delta^{2}+163.15103 \delta^{3}} \text { if } 0<\delta<0.3 \\
0.11441+0.92002 / \delta^{-0.39951 / \delta^{2}+0.05837 / \delta^{3}} \quad \text { if } 0.3 \leq \delta \leq 1
\end{array}\right.
$$




$$
d= \begin{cases}0.9718 & \text { if } 0<\delta<0.3 \\ 1.030 & \text { if } 0.3 \leq \delta \leq 1\end{cases}
$$

Figures 4.25 and 4.26 present the value of $c$ along with the equation 4.6 , which is the curve-fit for the constant $c$. The results from Table 4.6 show that the constant $d$ changes only slightly over the range of $\delta$ considered.

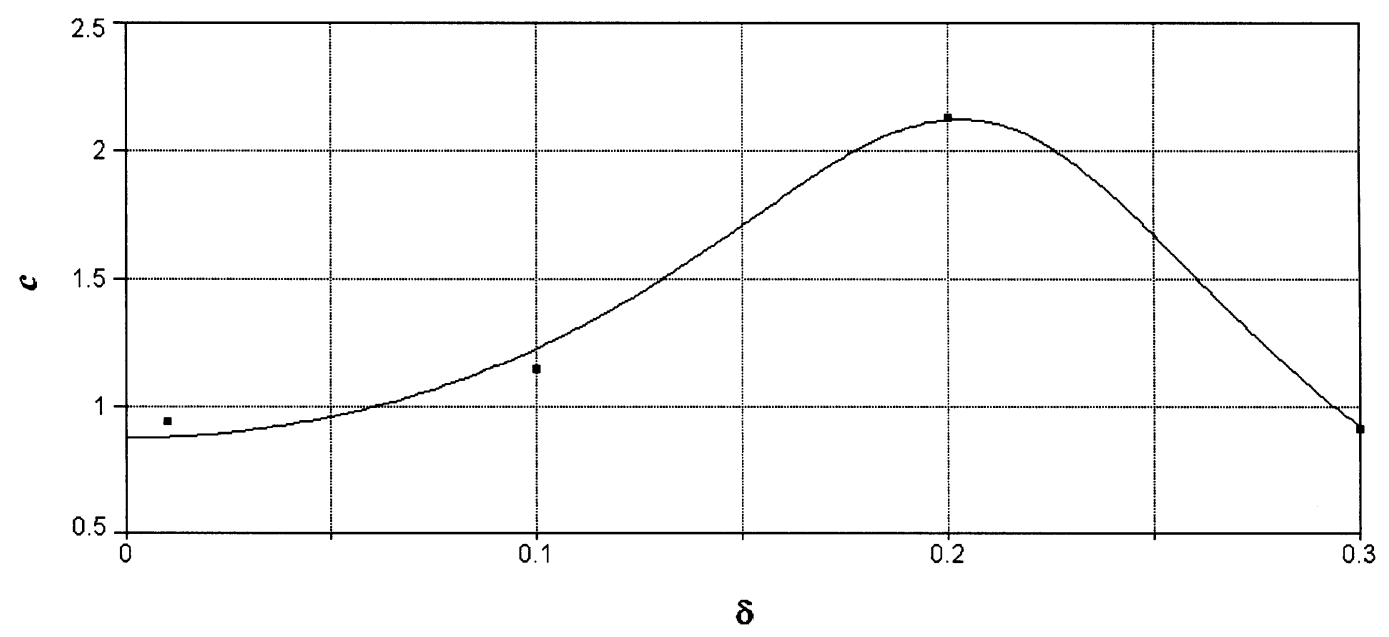

Figure 4.25: The curve-fitted (equation 4.6) to the constant $c$ where $0<\delta<0.3$ 


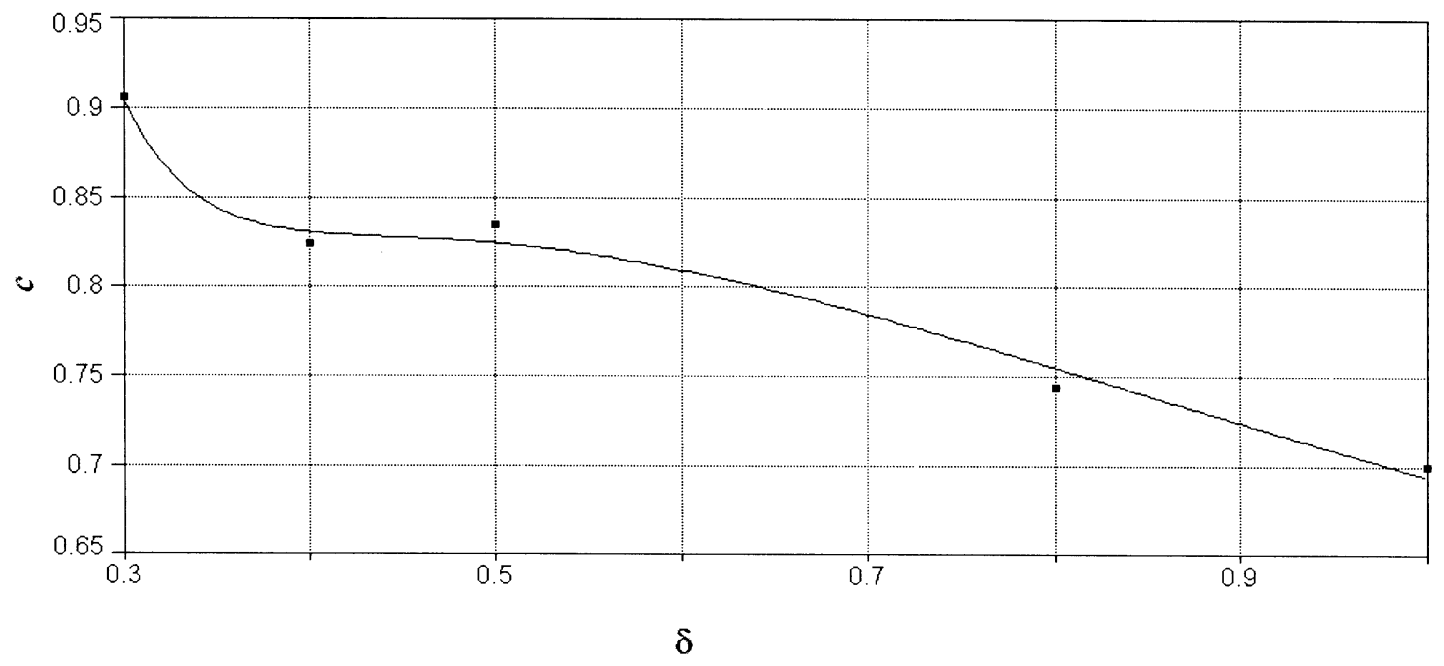

Figure 4.26: The curve-fitted (Equation 4.2) to the constant $c$ where $1 \leq \delta \leq 0.3$

Table 4.8 shows the comparison between the values of $c$ and $d$ with the Equations 4.6 and 4.7. Table 4.8 shows that the maximum percentage error is about $7 \%$, which happens for $\delta=0.1$.

Table 4.8: constant $c$ and $d$ for $\theta_{\mathrm{o}}=45^{\circ}$

\begin{tabular}{ccccccc}
\hline $\boldsymbol{\delta}$ & $\boldsymbol{c}$ & $\begin{array}{c}\boldsymbol{c} \\
(\mathbf{E q . ~ 4 . 6 )}\end{array}$ & \% Error & $\boldsymbol{d}$ & $\begin{array}{c}\boldsymbol{d} \\
(\mathbf{E q . ~ 4 . 7 )}\end{array}$ & \% Error \\
\hline 1 & 0.6992 & 0.6933 & $0.85 \%$ & 1.0435 & 1.0300 & $1.29 \%$ \\
0.8 & 0.7436 & 0.7542 & $-1.43 \%$ & 1.0373 & 1.0300 & $0.70 \%$ \\
0.5 & 0.8337 & 0.8234 & $1.24 \%$ & 1.0233 & 1.0300 & $-0.65 \%$ \\
0.4 & 0.8229 & 0.8295 & $-0.81 \%$ & 1.0447 & 1.0300 & $1.41 \%$ \\
0.3 & 0.9049 & 0.9039 & $0.11 \%$ & 1.0239 & 1.0300 & $-0.60 \%$ \\
0.2 & 2.1270 & 2.1165 & $0.49 \%$ & 0.9809 & 0.9718 & $0.93 \%$ \\
0.1 & 1.1404 & 1.2192 & $-6.91 \%$ & 1.0233 & 0.9718 & $5.03 \%$ \\
0.01 & 0.9335 & 0.8705 & $6.75 \%$ & 0.9683 & 0.9718 & $-0.36 \%$ \\
\hline
\end{tabular}


The comparison between the Nusselt number from the present model and Equation 4.4 shows that Equation 4.4 over predicts the Nusselt number for small Grashof numbers. At some gap-to-radius ratios this error is greater than $20 \%$. The comparison between Equation 4.4 and the present model for all gap cavities could be found in Appendix A. Table 4.9 presents a sample comparison between Equation 4.4 and the present model for $\delta=0.3$.

Table 4.9: The Nusselt number comparison between present model and Equation 4.4 for $\theta_{0}=45^{\circ} \delta=0.3$

\begin{tabular}{ccccc}
\hline $\mathbf{G r}$ & Ra* $^{1 / 4}$ & $\begin{array}{c}\text { Nu } \\
(\text { Present Model })\end{array}$ & $\begin{array}{c}\text { Nu } \\
(\mathbf{E q . 4 . 4})\end{array}$ & \% Error \\
\hline $1.0 \mathrm{E}+03$ & 1.2847 & 1.0423 & 1.1835 & $-13.55 \%$ \\
$2.5 \mathrm{E}+03$ & 1.6154 & 1.2602 & 1.4985 & $-18.90 \%$ \\
\hline $5.0 \mathrm{E}+03$ & 1.9211 & 1.7559 & 1.7913 & $-2.01 \%$ \\
$7.5 \mathrm{E}+03$ & 2.1260 & 2.0467 & 1.9884 & $2.85 \%$ \\
$1.0 \mathrm{E}+04$ & 2.2846 & 2.2479 & 2.1413 & $4.74 \%$ \\
$2.5 \mathrm{E}+04$ & 2.8727 & 2.9125 & 2.7111 & $6.92 \%$ \\
$5.0 \mathrm{E}+04$ & 3.4162 & 3.4787 & 3.2409 & $6.84 \%$ \\
$7.5 \mathrm{E}+04$ & 3.7807 & 3.8410 & 3.5975 & $6.34 \%$ \\
$1.0 \mathrm{E}+05$ & 4.0626 & 4.1121 & 3.8741 & $5.79 \%$ \\
$2.5 \mathrm{E}+05$ & 5.1084 & 5.0449 & 4.9051 & $2.77 \%$ \\
$5.0 \mathrm{E}+05$ & 6.0750 & 5.6078 & 5.8636 & $-4.56 \%$ \\
$7.5 \mathrm{E}+05$ & 6.7231 & 6.1987 & 6.5089 & $-5.00 \%$ \\
$1.0 \mathrm{E}+06$ & 7.2244 & 6.6655 & 7.0093 & $-5.16 \%$ \\
\hline $2.5 \mathrm{E}+06$ & 9.0842 & 8.4066 & 8.8745 & $-5.57 \%$ \\
\hline $5.0 \mathrm{E}+06$ & 10.8030 & 9.9787 & 10.6087 & $-6.31 \%$ \\
$7.5 \mathrm{E}+06$ & 11.9555 & 11.0876 & 11.7762 & $-6.21 \%$ \\
$1.0 \mathrm{E}+07$ & 12.8470 & 11.9219 & 12.6817 & $-6.37 \%$ \\
\hline
\end{tabular}


The average percentage errors for $\theta_{0}=45^{\circ}$ in all gap cavities are presented in Table 4.10. It can be concluded that the average percentage error between the correlation equation and the numerical data is less than $9 \%$.

Table 4.10: The average percentage error in Nusselt number calculated from Equation 4.4 and present model for $\theta_{0}=45^{\circ}$

\begin{tabular}{cc}
\hline $\boldsymbol{\delta}$ & Ave Error \\
\hline 1 & $3.43 \%$ \\
0.8 & $1.86 \%$ \\
0.5 & $2.92 \%$ \\
0.4 & $3.36 \%$ \\
0.3 & $6.46 \%$ \\
0.2 & $5.96 \%$ \\
0.1 & $8.52 \%$ \\
0.01 & $5.45 \%$ \\
\hline
\end{tabular}

Figure 4.27 shows a graphical comparison between the Nusselt number calculated from the present model and Equation 4.4 with $\pm 10 \%$ error bars for Equation 4.4. 


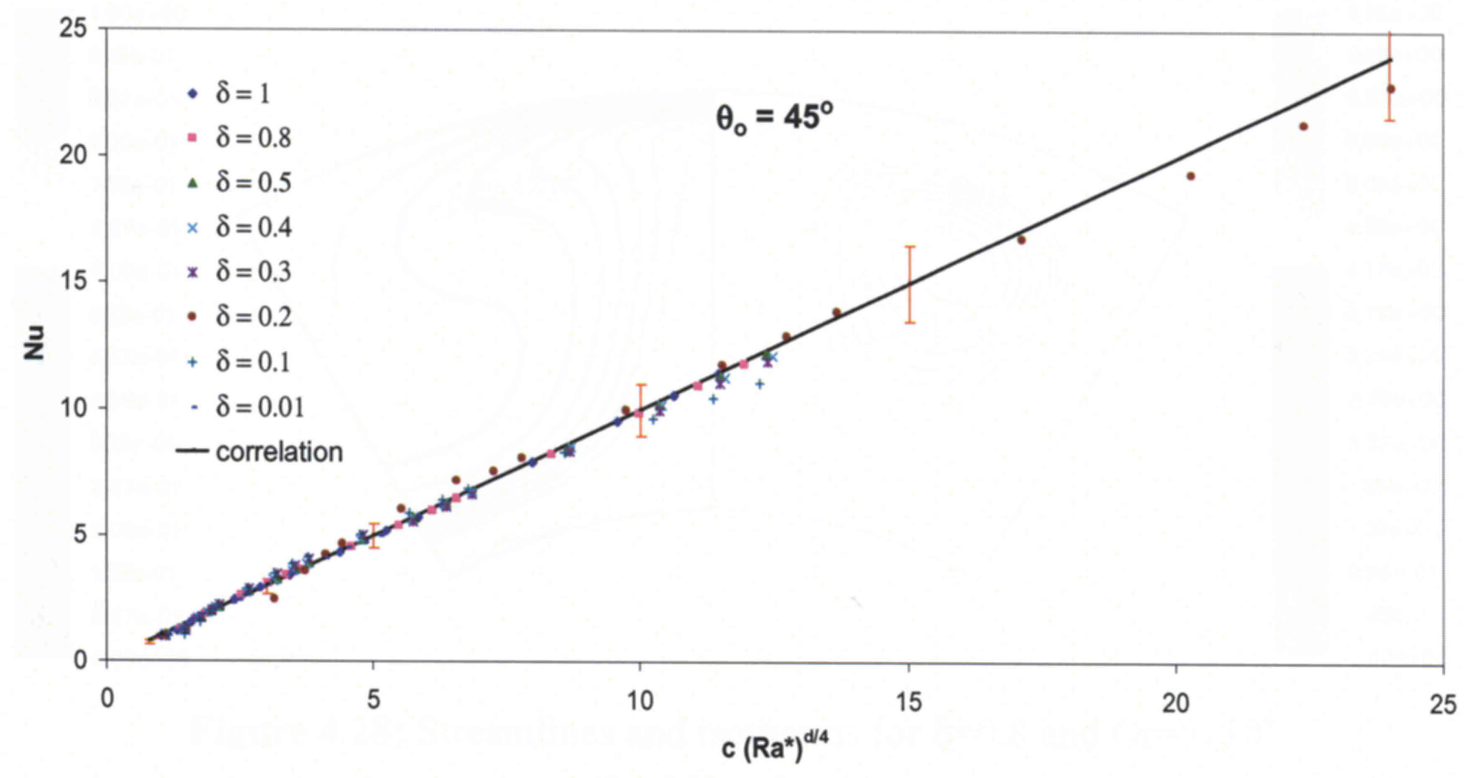

Figure 4.27: Nusselt number correlation with $\pm 10 \%$ error bars and numerical results for $\theta_{0}=45^{\circ}$.

\subsubsection{Concentric cavities with Truncation Angle of $\left(\theta_{0}=30^{\circ}\right)$}

Figures 4.28 to 4.33 compare the streamlines and isotherms in the cavity for a truncation angle of $30^{\circ}$. The numerical results converged to the steady state for large and moderate gap-to-radius ratios over the full range of Grashof numbers considered in this study. The numerical results show that the fluid flow is made of a single vortex-cell. This vortex-cell becomes larger as the Grashof number increases. Figures 4.28 and 4.29 show the isotherms and streamlines for cavities with large gap ratios and Figures 4.30 and 4.31 show the isotherms and streamlines for moderate gap ratios at Grashof numbers of $5 \times 10^{4}$ and $5 \times 10^{6}$, respectively. 

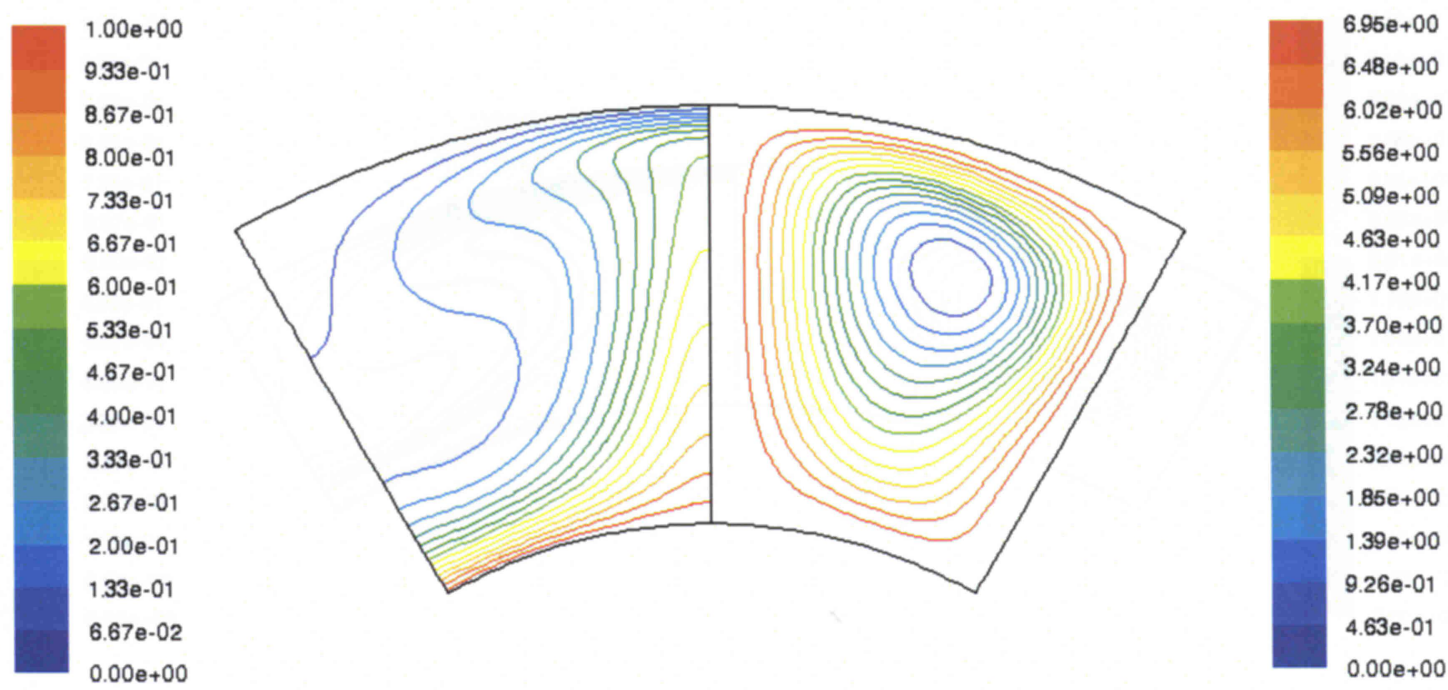

Figure 4.28: Streamlines and isotherms for $\delta=0.8$ and $\mathrm{Gr}=5 \times 10^{4}$ $\left(\theta_{0}=30^{\circ}\right.$ and $\left.\varepsilon=0\right)$

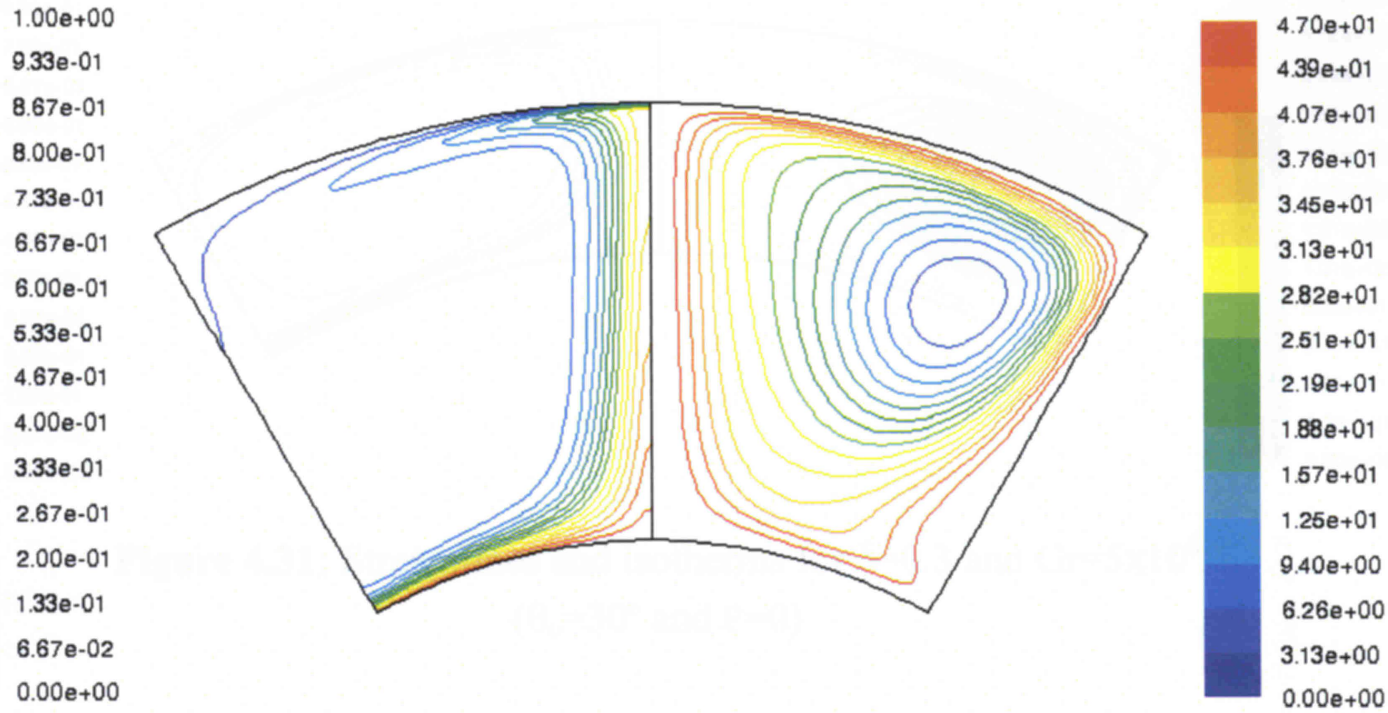

Figure 4.29: Streamlines and isotherms for $\delta=0.8$ and $\mathrm{Gr}=5 \times 10^{6}$

$$
\left(\theta_{0}=30^{\circ} \text { and } \varepsilon=0\right)
$$






Figure 4.30: Streamlines and isotherms for $\delta=0.3$ and $\mathrm{Gr}=5 \times 10^{4}$

$$
\left(\theta_{0}=30^{\circ} \text { and } \varepsilon=0\right)
$$


Figure 4.31: Streamlines and isotherms for $\delta=0.3$ and $\mathrm{Gr}=5 \times 10^{6}$

$$
\left(\theta_{0}=30^{\circ} \text { and } \varepsilon=0\right. \text { ) }
$$

Figures 4.32 and 4.33 present the streamlines and isotherms in cavities with small gap ratios. At small Grashof numbers, the numerical results converged to a steady state condition, with a single vortex-cell. At moderate and high Grashof numbers, multiple vortex-cells were observed. The fluid flow converged to steady state conditions for all 
Grashof numbers, except at the smallest gap ratio $(\delta=0.01)$. For the gap ratio of 0.01 similar to the moderate gap ratios, oscillation in the Nusselt number was observed.

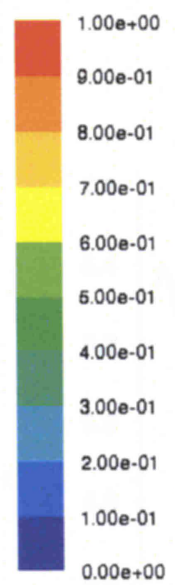

Figure 4.32: Streamlines and isotherms for $\delta=0.1$ and $\mathrm{Gr}=5 \times 10^{4}$ $\left(\theta_{0}=30^{\circ}\right.$ and $\left.\varepsilon=0\right)$

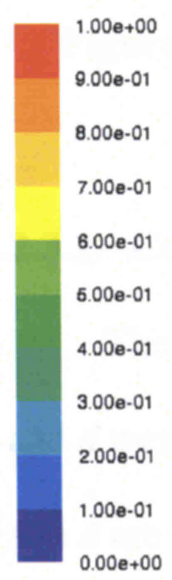

Figure 4.33: Streamlines and isotherms for $\delta=0.1$ and $\mathrm{Gr}=5 \times 10^{6}$

$$
\left(\theta_{0}=30^{\circ} \text { and } \varepsilon=0\right)
$$


At some Grashof numbers, periodic behavior was not observed. For instance,

Figure 4.34 shows the oscillation in Nusselt number in the gap cavity $\delta=0.01$ for a Grashof number of $5 \times 10^{6}$.



Figure 4.34: Oscillation in Nusselt number when Time Step $=0.01, \delta=0.01$ and

$$
\mathrm{Gr}=5 \times 10^{6}\left(\theta_{\mathrm{o}}=30^{\circ} \text { and } \varepsilon=0\right)
$$

\subsubsection{Correlations for Heat Transfer}

The same steps were followed as for the other truncation angles, and the constants $c$ and $d$ were found from curve fitting Equation 4.1 to the numerical results. The values of $c$ and $d$ are presented in Table 4.11. 
Table 4.11: The constant $c$ and $d$ for $\theta_{0}=30^{\circ}$

\begin{tabular}{ccc}
\hline $\boldsymbol{\delta}$ & $\boldsymbol{c}$ & $\boldsymbol{d}$ \\
\hline 1 & 0.5173 & 1.1585 \\
0.8 & 0.5956 & 1.1174 \\
0.5 & 0.7650 & 1.0526 \\
0.4 & 0.8465 & 1.0271 \\
0.3 & 0.8815 & 1.0373 \\
0.2 & 1.1275 & 0.9511 \\
0.1 & 1.3497 & 0.9166 \\
0.01 & 1.4710 & 0.8279 \\
\hline
\end{tabular}

Figure 4.35 shows a graphical comparison between the Equation 4.1 (with $\pm 4 \%$ error bars) and the numerical results for $\delta=0.2$.

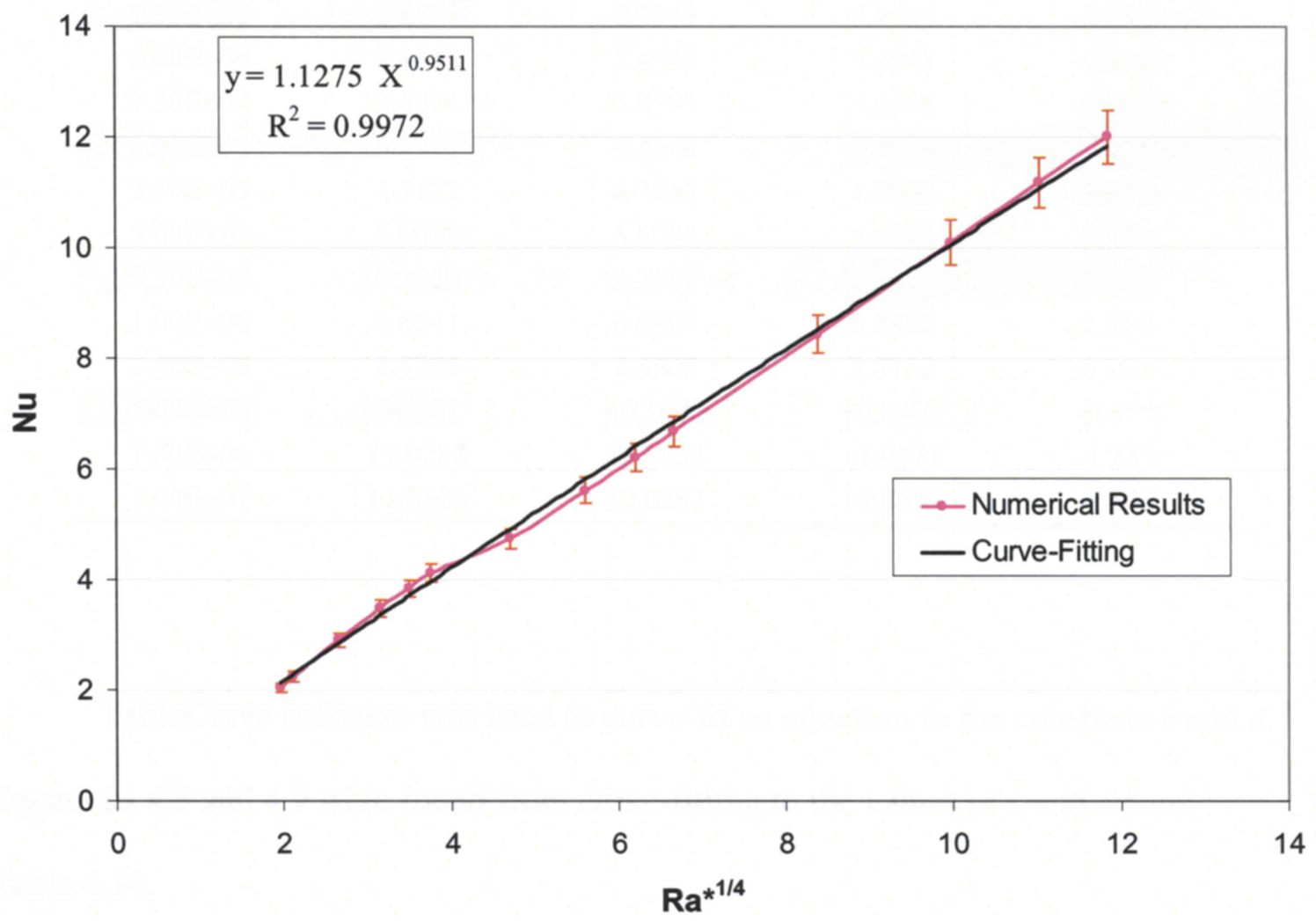

Figure 4.35: Nusselt number from numerical result ( $\pm 4 \%$ error bar) and power function fitted to the numerical results for $\delta=0.2$ 
Table 4.12 compares these values. From Table 4.12, it can be concluded that, for small Grashof numbers, Equation 4.1 over predicts the Nusselt number by more than 29\%. Except for the first two Nusselt number at very low Grashof numbers, Equation 4.1 agrees well with the Nusselt number found from the present model.

Table 4.12: Nusselt number calculated from present model and power function curve-fitting for $\delta=0.2$

\begin{tabular}{ccccc}
\hline $\mathbf{G r}$ & $\mathbf{R a}^{* \mathbf{1 / 4}}$ & $\begin{array}{c}\text { Nu } \\
\text { (Present Model) }\end{array}$ & $\begin{array}{c}\text { Nu* } \\
\text { Curve Fitting) }\end{array}$ & \% Error \\
\hline $1.00 \mathrm{E}+03$ & 1.1851 & 1.0239 & 1.3251 & $29.42 \%$ \\
$2.50 \mathrm{E}+03$ & 1.4901 & 1.2270 & 1.6477 & $34.28 \%$ \\
$5.00 \mathrm{E}+03$ & 1.7721 & 1.7521 & 1.9429 & $10.89 \%$ \\
$7.50 \mathrm{E}+03$ & 1.9611 & 2.0509 & 2.1395 & $4.32 \%$ \\
$1.00 \mathrm{E}+04$ & 2.1074 & 2.2552 & 2.2910 & $1.59 \%$ \\
$2.50 \mathrm{E}+04$ & 2.6499 & 2.9249 & 2.8487 & $-2.60 \%$ \\
$5.00 \mathrm{E}+04$ & 3.1512 & 3.4948 & 3.3591 & $-3.88 \%$ \\
$7.50 \mathrm{E}+04$ & 3.4874 & 3.8593 & 3.6991 & $-4.15 \%$ \\
$1.00 \mathrm{E}+05$ & 3.7475 & 4.1302 & 3.9610 & $-4.10 \%$ \\
$2.50 \mathrm{E}+05$ & 4.7122 & 4.7537 & 4.9252 & $3.61 \%$ \\
$5.00 \mathrm{E}+05$ & 5.6038 & 5.6294 & 5.8076 & $3.17 \%$ \\
$7.50 \mathrm{E}+05$ & 6.2016 & 6.2193 & 6.3954 & $2.83 \%$ \\
$1.00 \mathrm{E}+06$ & 6.6641 & 6.6801 & 6.8482 & $2.52 \%$ \\
$2.50 \mathrm{E}+06$ & 8.3796 & 8.4509 & 8.5152 & $0.76 \%$ \\
$5.00 \mathrm{E}+06$ & 9.9651 & 10.1070 & 10.0409 & $-0.65 \%$ \\
$7.50 \mathrm{E}+06$ & 11.0282 & 11.1953 & 11.0571 & $-1.23 \%$ \\
$1.00 \mathrm{E}+07$ & 11.8506 & 12.0257 & 11.8399 & $-1.54 \%$ \\
\hline
\end{tabular}

TableCurve software was used to curve-fit an equation to the constants $c$ and $d$. Equations 4.8 and 4.9 were found from curve-fitting to the values of $c$ and $d$ from Table 4.11. 


$$
\begin{aligned}
c^{-1}= & 0.68665-0.76732 \delta+16.73474 \delta^{2}-46.54738 \delta^{3} \\
& +52.44906 \delta^{4}-20.62163 \delta^{5}
\end{aligned}
$$

The Equation 4.8 and 4.9 curve-fitted to the value of $c$ and $d$ are shown in Figures 4.36 and 4.37 , respectively.



Figure 4.36: The curve-fitted (Equation 4.8) to the constant $c$ 


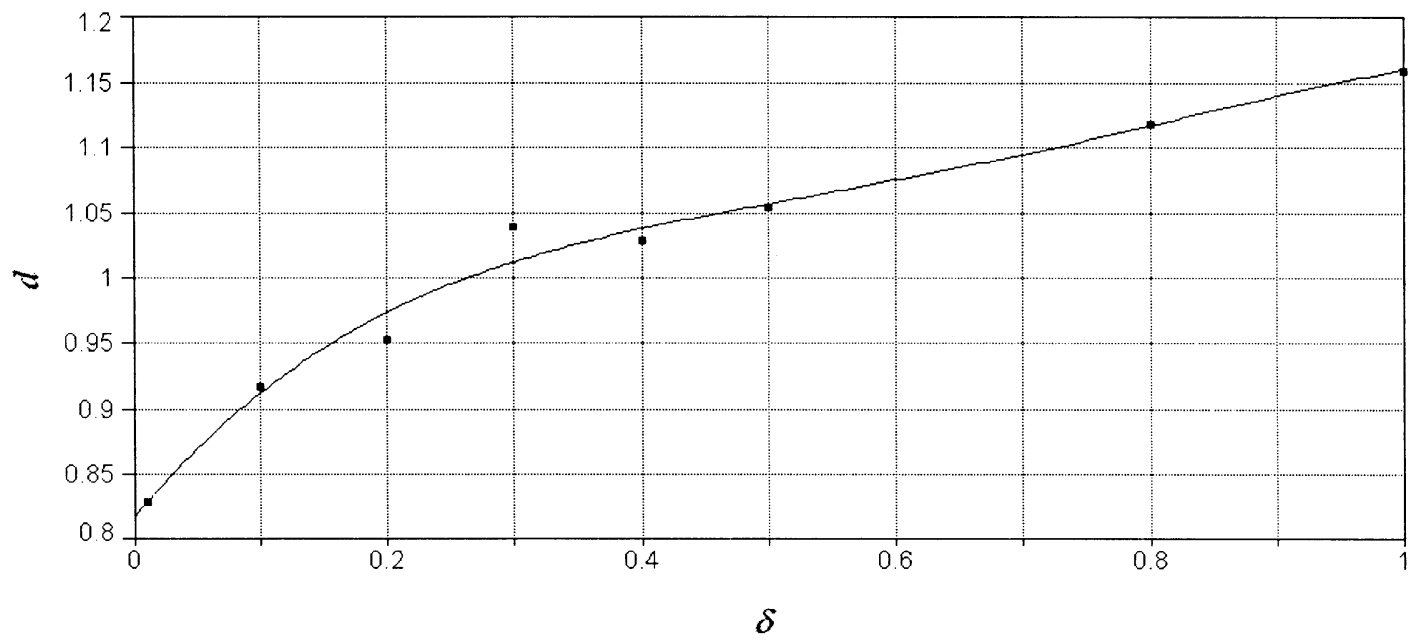

Figure 4.37: The curve-fitted (Equation 4.9) to the constant $d$

Table 4.13 compares the values of $\mathrm{c}$ and $\mathrm{d}$ from Table 4.11 and Equations 4.8 and 4.9. Table 4.13 shows that Equations 4.8 and 4.9 predict the values of $c$ and $d$ with less than $5 \%$ error.

Table 4.13: constant $c$ and $d$ for $\theta_{0}=30^{\circ}$

\begin{tabular}{ccccccc}
\hline $\boldsymbol{\delta}$ & $\boldsymbol{c}$ & $\boldsymbol{c}$ & \% Error & $\boldsymbol{d}$ & $\begin{array}{c}\boldsymbol{d} \\
(\mathbf{E q ~ 4 . 9 )})\end{array}$ & \% Error \\
\hline 1 & 0.5173 & 0.5170 & $0.05 \%$ & 1.1585 & 1.1589 & $-0.04 \%$ \\
0.8 & 0.5956 & 0.5964 & $-0.14 \%$ & 1.1174 & 1.1157 & $0.15 \%$ \\
0.5 & 0.7650 & 0.7681 & $-0.41 \%$ & 1.0526 & 1.0556 & $-0.29 \%$ \\
0.4 & 0.8465 & 0.8266 & $2.35 \%$ & 1.0271 & 1.0363 & $-0.89 \%$ \\
0.3 & 0.8815 & 0.9255 & $-4.99 \%$ & 1.0373 & 1.0108 & $2.56 \%$ \\
0.2 & 1.1275 & 1.1019 & $2.27 \%$ & 0.9511 & 0.9721 & $-2.21 \%$ \\
0.1 & 1.3497 & 1.3591 & $-0.70 \%$ & 0.9166 & 0.9112 & $0.59 \%$ \\
0.01 & 1.4710 & 1.4693 & $0.12 \%$ & 0.8279 & 0.8278 & $0.01 \%$ \\
\hline
\end{tabular}


Therefore, the Nusselt number calculated from the present model can be estimated with Equation 4.4, where the constants $c$ and $d$ are calculated from Equations 4.8 and 4.9, respectively. Table 4.14 shows a comparison between the Nusselt number calculated from the present model and the Nusselt number calculated from Equation 4.4 for a gap ratio equal to 0.3 . Table 4.14 shows that Equation 4.4 can not predict the Nusselt number closely for Grashof numbers less than $2.5 \times 10^{3}$.

Table 4.14: The Nusselt number comparison between present model and Equation 4.4 for $\theta_{0}=30^{\circ} \delta=0.3$

\begin{tabular}{ccccc}
\hline $\mathbf{G r}$ & $\mathbf{R a}^{* \mathbf{1 / 4}}$ & $\begin{array}{c}\mathbf{N u} \\
(\text { Present Model })\end{array}$ & $\begin{array}{c}\mathbf{N u} \\
(\mathbf{E q . ~ 4 . 4})\end{array}$ & \% Error \\
\hline $1.0 \mathrm{E}+03$ & 1.1851 & 1.0239 & 1.2997 & $-26.93 \%$ \\
$2.5 \mathrm{E}+03$ & 1.4901 & 1.2270 & 1.6238 & $-32.34 \%$ \\
$5.0 \mathrm{E}+03$ & 1.7721 & 1.7521 & 1.9218 & $-9.68 \%$ \\
$7.5 \mathrm{E}+03$ & 1.9611 & 2.0509 & 2.1208 & $-3.41 \%$ \\
$1.0 \mathrm{E}+04$ & 2.1074 & 2.2552 & 2.2744 & $-0.85 \%$ \\
$2.5 \mathrm{E}+04$ & 2.6499 & 2.9249 & 2.8417 & $2.84 \%$ \\
$5.0 \mathrm{E}+04$ & 3.1512 & 3.4948 & 3.3631 & $3.77 \%$ \\
$7.5 \mathrm{E}+04$ & 3.4874 & 3.8593 & 3.7114 & $3.83 \%$ \\
$1.0 \mathrm{E}+05$ & 3.7475 & 4.1302 & 3.9802 & $3.63 \%$ \\
$2.5 \mathrm{E}+05$ & 4.7122 & 4.7537 & 4.9730 & $-4.61 \%$ \\
$5.0 \mathrm{E}+05$ & 5.6038 & 5.6294 & 5.8854 & $-4.55 \%$ \\
$7.5 \mathrm{E}+05$ & 6.2016 & 6.2193 & 6.4949 & $-4.43 \%$ \\
$1.0 \mathrm{E}+06$ & 6.6641 & 6.6801 & 6.9653 & $-4.27 \%$ \\
$2.5 \mathrm{E}+06$ & 8.3796 & 8.4509 & 8.7027 & $-2.98 \%$ \\
$5.0 \mathrm{E}+06$ & 9.9651 & 10.1070 & 10.2995 & $-1.90 \%$ \\
$7.5 \mathrm{E}+06$ & 11.0282 & 11.1953 & 11.3661 & $-1.53 \%$ \\
$1.0 \mathrm{E}+07$ & 11.8506 & 12.0257 & 12.1893 & $-1.36 \%$ \\
\hline
\end{tabular}


The average error in Nusselt number, over all Grashof numbers considered, is calculated using Equation 4.4 for each gap ratio is presented in Table 4.15. It can be concluded that the average percentage error increases for small gap ratios, and the maximum error happens for a gap ratio equal to 0.01 .

Table 4.15: The average percentage error in Nusselt number calculated from Equation 4.4 and the present model for $\theta_{0}=30^{\circ}$

\begin{tabular}{cc}
\hline $\boldsymbol{\delta}$ & $\begin{array}{c}\text { Average } \\
\text { \% Error }\end{array}$ \\
\hline 1 & $1.68 \%$ \\
0.8 & $1.60 \%$ \\
0.5 & $3.07 \%$ \\
0.4 & $3.44 \%$ \\
0.3 & $3.37 \%$ \\
0.2 & $6.64 \%$ \\
0.1 & $8.14 \%$ \\
0.01 & $10.69 \%$ \\
\hline
\end{tabular}

\subsubsection{Discussion}

The fluid flow in domed concentric cavities with a uniform gap thickness was found to be steady for large gap ratios. The flow could be steady or unsteady for moderate gap ratios. Fluid flow in cavities with moderate and large gap-to-radius ratios $(\delta<0.5)$ might contain a single-vortex-cell or multiple-vortex-cells. The fluid flow in small gap cavities was found to be unsteady. At a truncation angle of $30^{\circ}$, for Grashof numbers larger than $10^{5}$, in some cases, the numerical results showed unsteady flow in the cavity.

Figures 4.38 to 4.40 compare the effect of gap cavity on Nusselt number for three truncation angles of $90^{\circ}, 45^{\circ}$ and $30^{\circ}$. It can be concluded that, for a truncation angle of 
$90^{\circ}$, changes in gap cavity have a small effect on Nusselt number, but at smaller truncation angles, the Nusselt number increases as the gap ratio decreases.

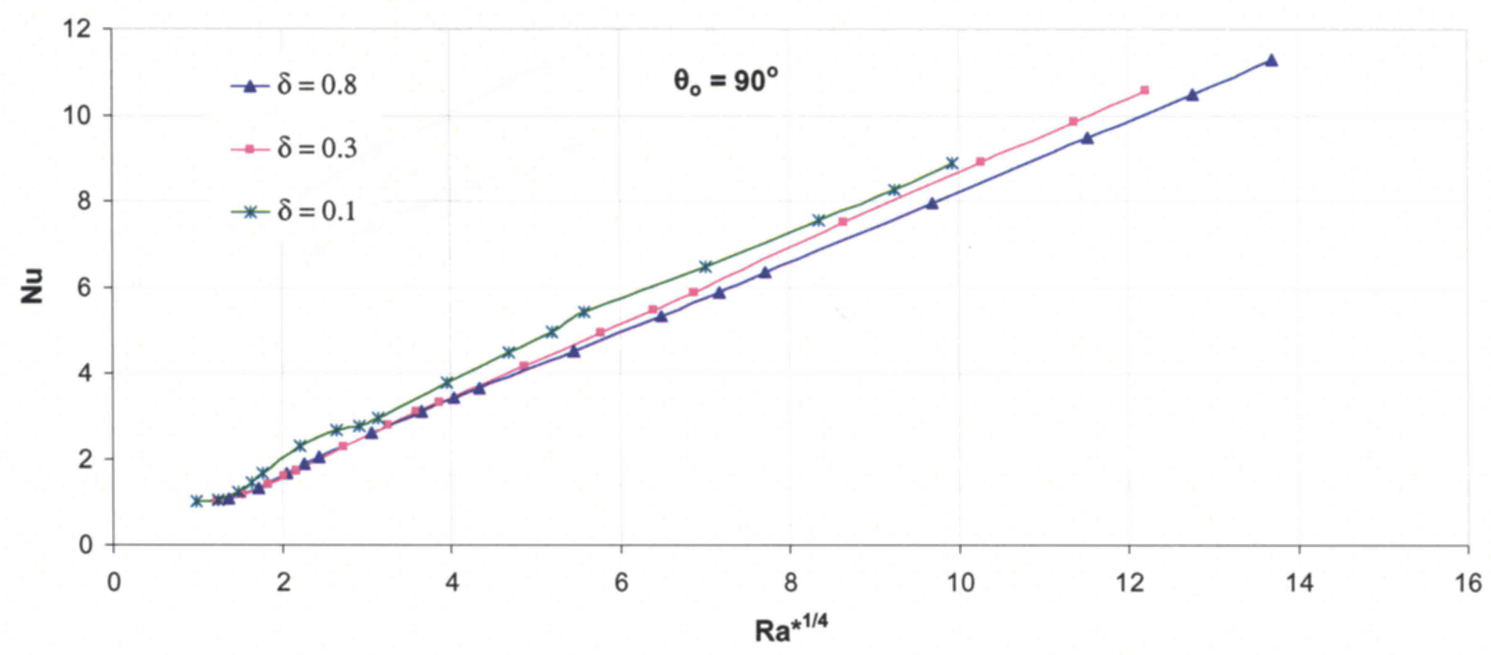

Figure 4.38: Profile of the time averaged Nusselt number as a function of $\mathrm{Ra}^{1 / 4}$ for a truncation angle of $90^{\circ}$

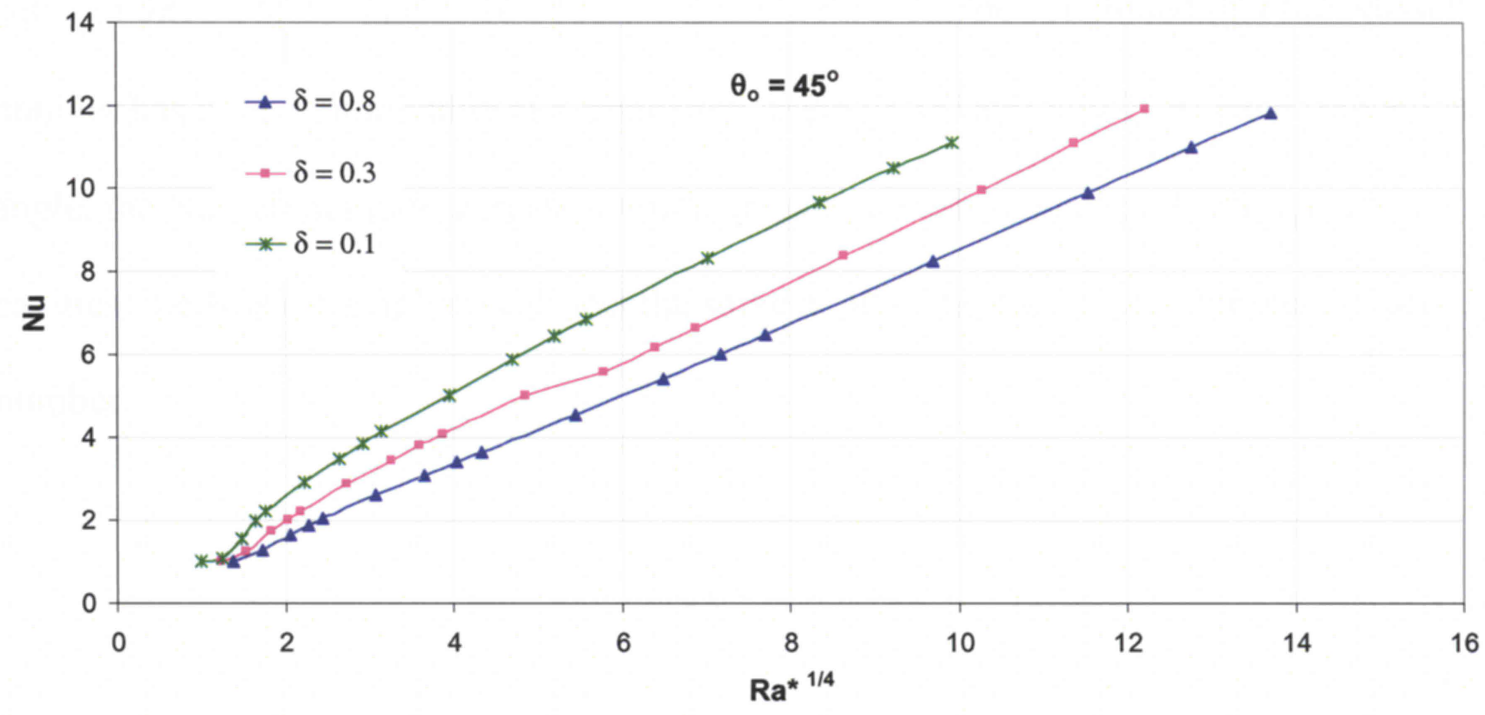

Figure 4.39: Profile of the time averaged Nusselt number as a function of $\mathrm{Ra}^{* 1 / 4}$ for a truncation angle of $45^{\circ}$ 


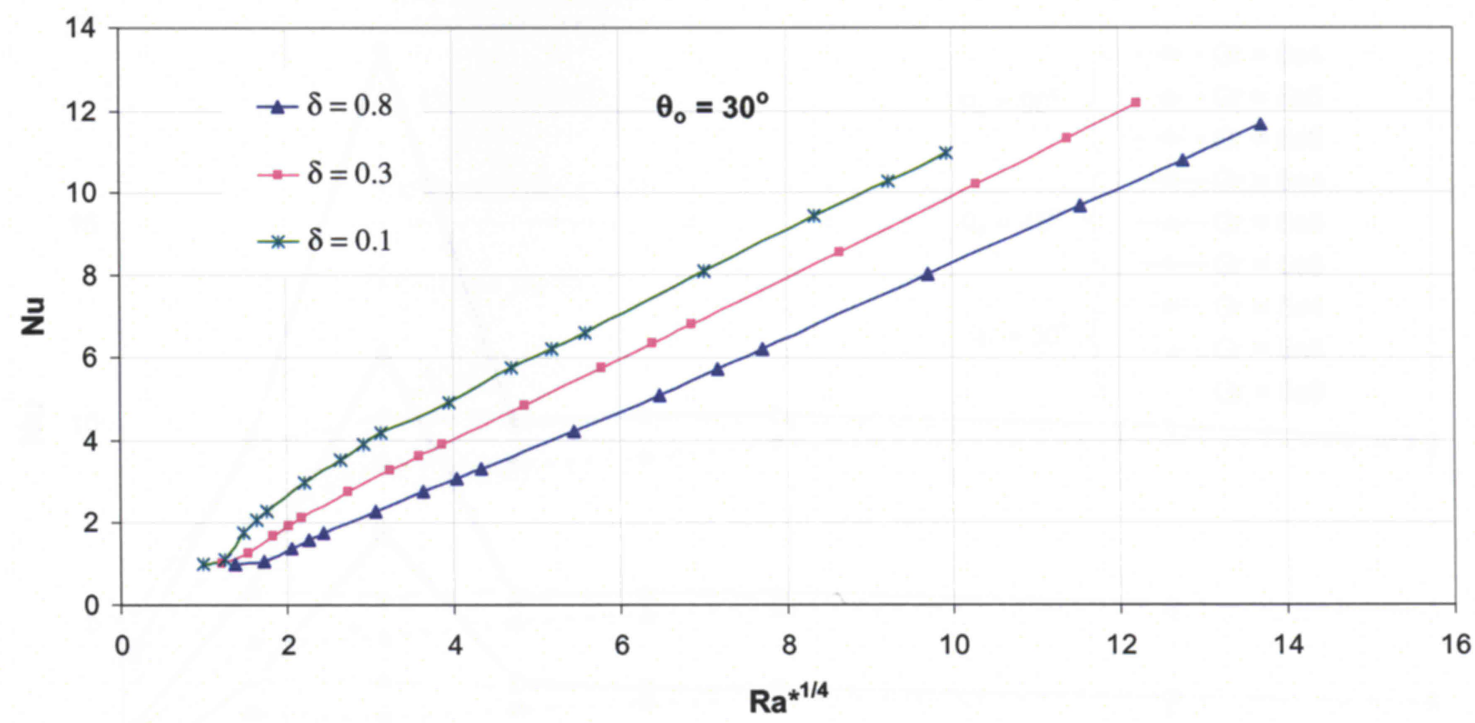

Figure 4.40: Profile of the time average Nusselt number as a function of $\mathrm{Ra}^{* 1 / 4}$ for a truncation angle of $30^{\circ}$

Figure 4.41 compares the Nusselt numbers for truncation angles of $90^{\circ}, 45^{\circ}$ and $30^{\circ}$. The Nusselt number for each truncation angle is presented for Grashof numbers equal to $5 \times 10^{4}, 5 \times 10^{5}$ and $5 \times 10^{6}$. From Figure 4.47 , it can be concluded that the Nusselt number has a maximum value at a truncation angle of $45^{\circ}$ and $\delta=0.2$. At each truncation angle, the Nusselt number increases with increasing Grashof number. For large gap cavities, the Nusselt number is almost the same at all truncation angles for each Grashof number. 


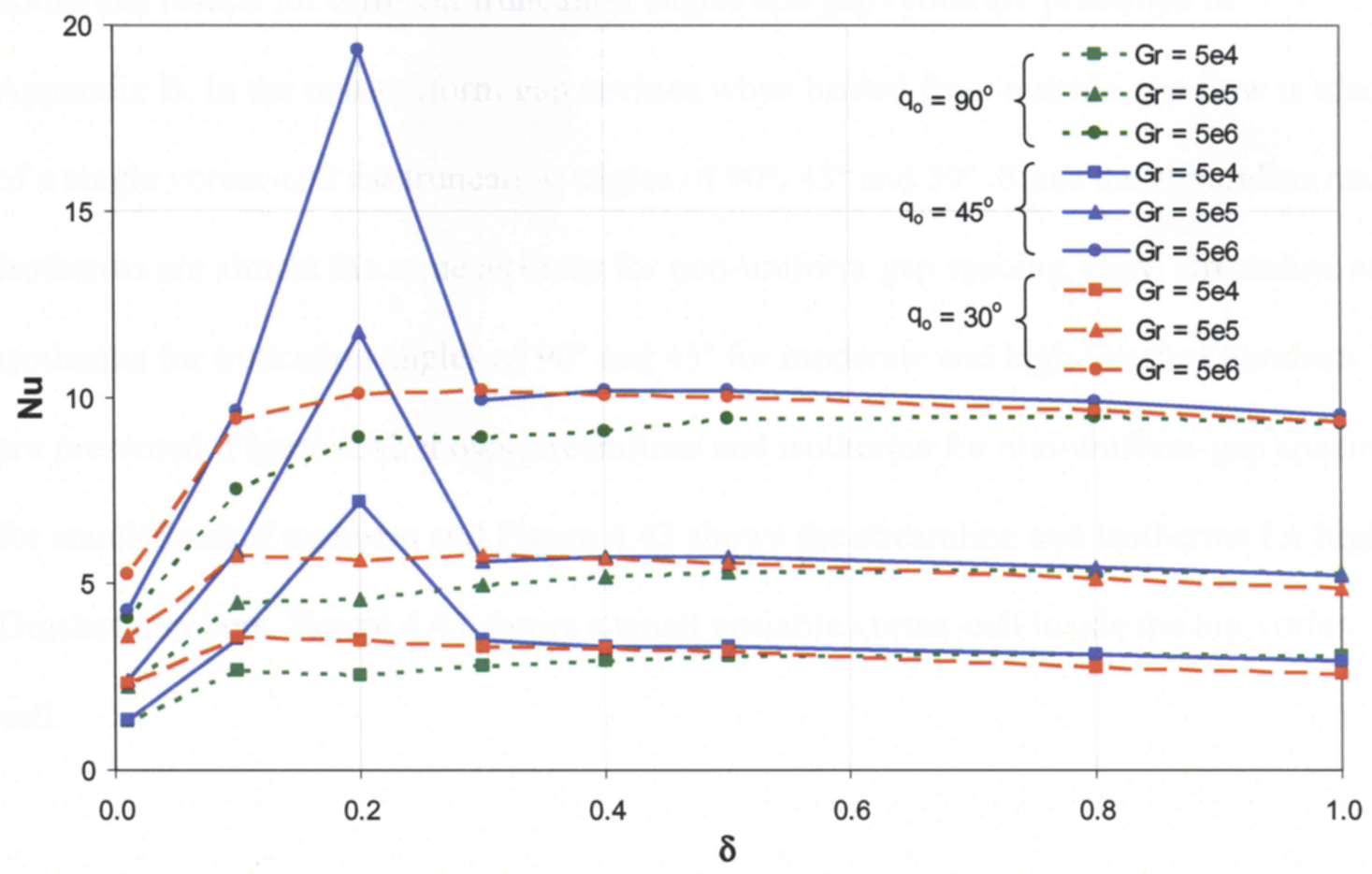

Figure 4.41: Nusselt number as a function of $\delta$

\subsection{Results for Eccentric Dome Cavities}

Domed eccentric cavities with non-uniform gap spacing were studied when heated from the outside surface. The numerical results show that the flow is stable. The numerical results reached steady-state with a single vortex-cell for small and moderate Grashof numbers, independently of the truncation angle. For large Grashof numbers (larger than $10^{6}$ ), small unstable vortex-cells formed inside the big vortex-cell. The unstable vortex-cells caused small oscillations in Nusselt number. In such cases, the Nusselt number was calculated from the time average over two or three time periods. The 
numerical results for different truncation angles and gap ratios are presented in Appendix B. In the non-uniform gap cavities when heated from outside, the flow is made of a single vortex-cell for truncation angles of $90^{\circ}, 45^{\circ}$ and $30^{\circ}$. Since the streamline and isotherms are almost the same as those for non-uniform gap spacing, only, streamline and isotherms for truncation angles of $90^{\circ}$ and $45^{\circ}$ for moderate and high Grashof numbers are presented. Figure 4.42 shows streamlines and isotherms for non-uniform-gap spacing for small Grashof numbers and Figure 4.43 shows the streamline and Isotherms for high Grashof numbers. Figure 4.43 shows a small unstable vortex-cell inside the big vortex cell. 

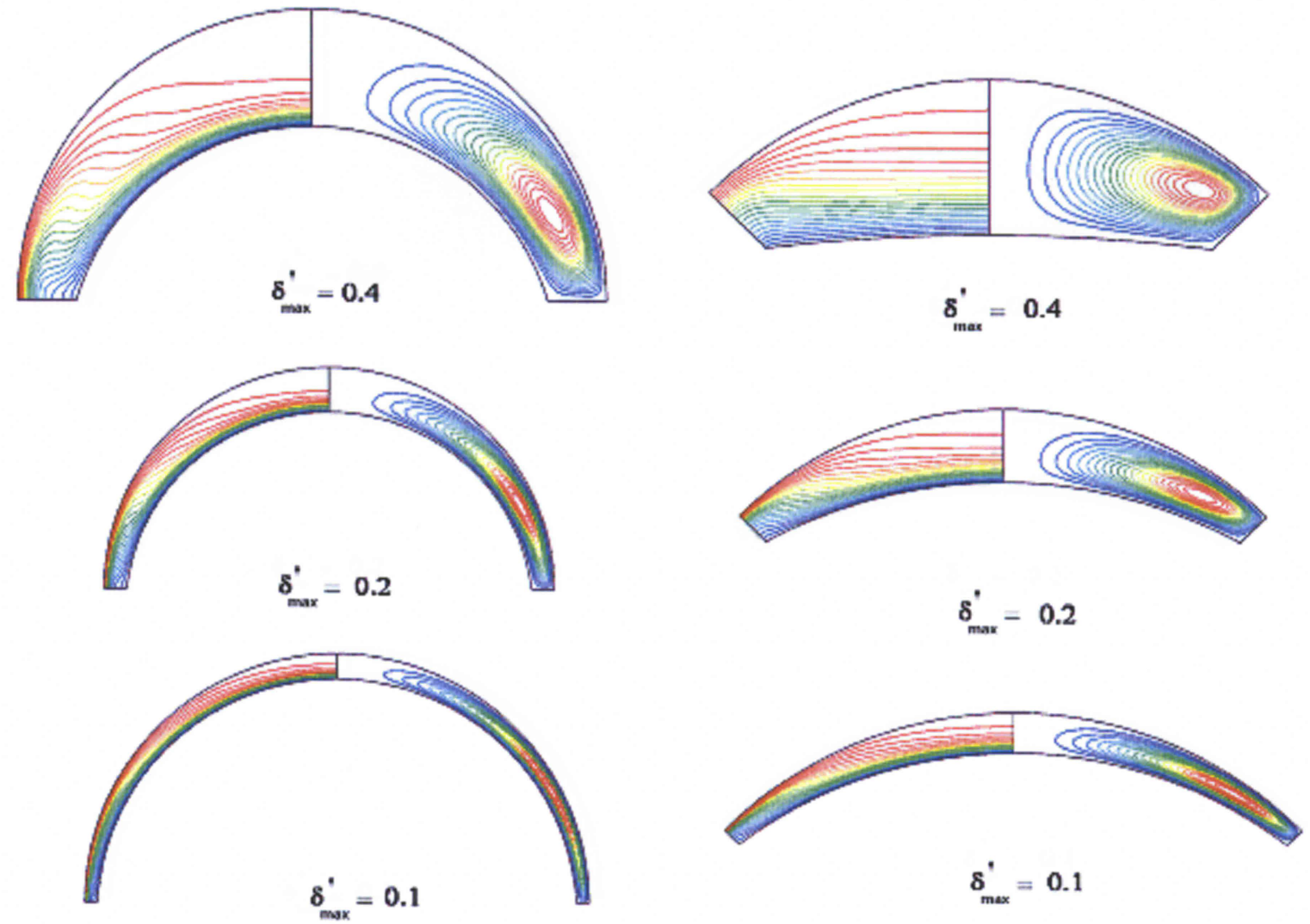

$$
\theta_{0}=90^{\circ}
$$

$$
\theta_{0}=45^{\circ}
$$

Figure 4.42: Streamline and isotherms for $\theta_{0}=45^{\circ}$ and $90^{\circ}$ when $\mathrm{Gr}=5 \times 10^{4}$ 

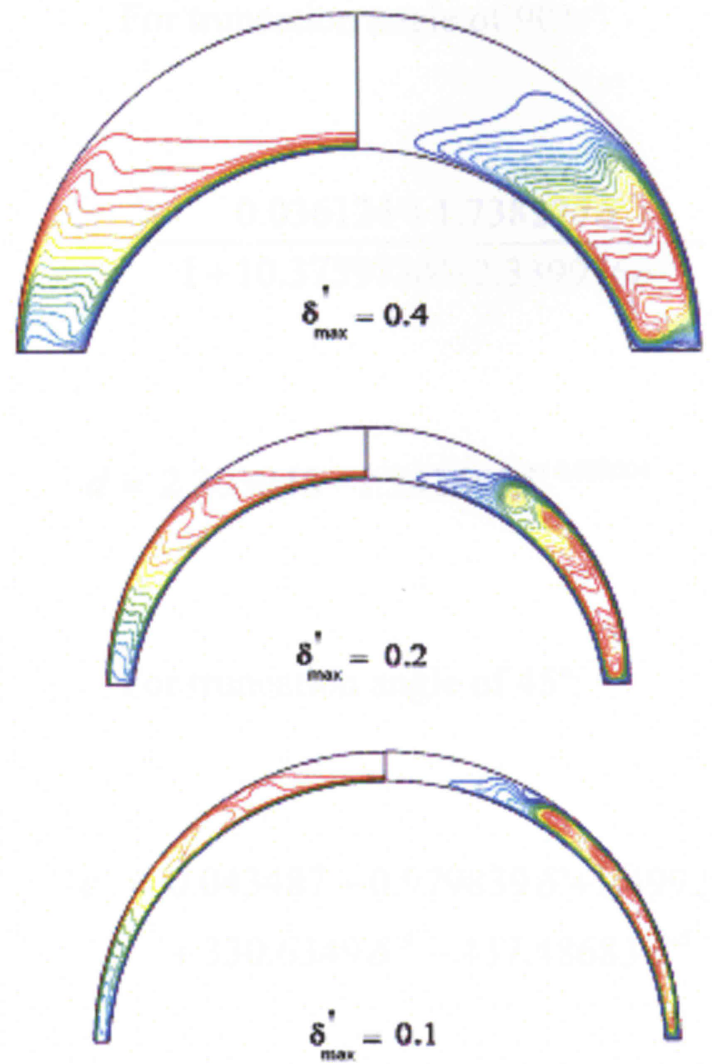

$\theta_{0}=90^{\circ}$
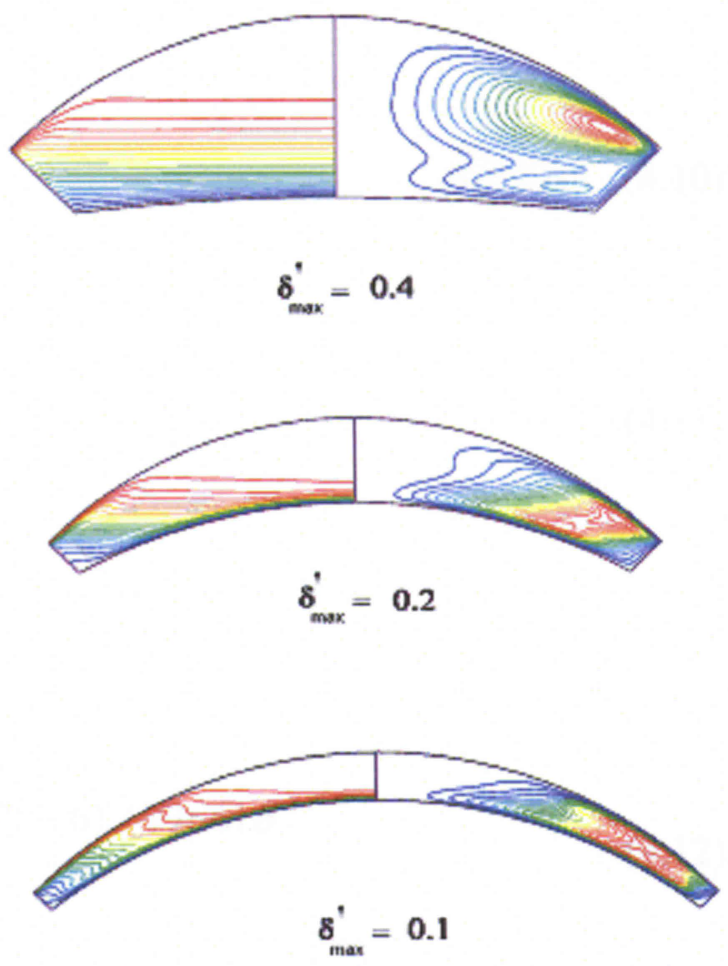

$$
\theta_{0}=45^{\circ}
$$

Figure 4.43: Streamline and isotherms for $\theta_{0}=45^{\circ}$ and $90^{\circ}$ when $\mathrm{Gr}=5 \times 10^{6}$

Since the procedure for curve-fitting the best-fit equation to the numerical results is the same as the procedure that was followed for uniform-gap thickness, only the final correlations are presented in this chapter. As for the uniform-gap thickness, the Nusselt number is defined with Equation 4.4. The coefficients $c$ and $d$ for the non-uniform gap thickness are found as follows: 
For truncation angle of $90^{\circ}$ :

$$
c=\frac{0.036124+1.738277 \delta^{\prime}}{1+10.375973 \delta^{\prime}+2.339976 \delta^{\prime 2}}
$$

$$
d=2.303048-1.343649 \delta^{0.023004}
$$

For truncation angle of $45^{\circ}$ :

$$
\begin{aligned}
c= & 0.043487+0.979839 \delta^{\prime}+1.499226 \delta^{\prime 2}-61.693641 \delta^{\prime 3} \\
& +330.6349 \delta^{\prime 4}-437.48683 \delta^{\prime 5} \\
d= & 1.056547+0.754604 \delta^{\prime}-41.676863 \delta^{\prime 2}+406.43125 \delta^{\prime 3} \\
& -1965.2146 \delta^{\prime 4}+4220.9736 \delta^{\prime 5}-3268.143 \delta^{\prime 6}
\end{aligned}
$$

For truncation angle of $30^{\circ}$

$$
c= \begin{cases}0.052475+0.605576 \delta^{\prime}+12.440602 \delta^{\prime 2}-37.396661 \delta^{\prime 3} & \text { when } \delta^{\prime}<0.1 \\ 0.5328-15.555 \delta^{\prime}+160.46 \delta^{\prime 2}-381.6 \delta^{\prime 3} & \text { when } \delta^{\prime} \geq 0.1\end{cases}
$$




$$
d= \begin{cases}\frac{1.044507+2.651547 \delta^{\prime}}{1+1.884586 \delta^{\prime}+63.377047 \delta^{\prime 2}} & \text { when } \delta^{\prime}<0.1 \\ \frac{0.610486-1.101862 \delta^{\prime}}{1-13.329955 \delta^{\prime}+102.9113 \delta^{\prime 2}} & \text { when } \delta^{\prime} \geq 0.1\end{cases}
$$

Equation 4.4 predicts the numerical results with less than $10 \%$ error except for the truncation angle of $30^{\circ}$ and $\delta^{\prime}=0.2$, where the error is $22.8 \%$ when the Grashof number is $10^{7}$. Table 4.16 shows the maximum absolute error for different truncation angles except for the Grashof number of $10^{7}$ when $\theta_{0}=30^{\circ}$ and $\delta^{\prime}=0.2$.

Table 4.16: The maximum absolute error in Equation 4.4 related the Numerical results

\begin{tabular}{cc}
\hline$\theta_{\mathbf{o}}$ & $\begin{array}{c}\text { Maximum Absolute } \\
\text { Error }\end{array}$ \\
\hline 30 & $8.32 \%$ \\
45 & $8.84 \%$ \\
90 & $9.75 \%$ \\
\hline
\end{tabular}

\subsubsection{Discussion}

The fluid flow in a double glazed domed skylight with a non-uniform gap thickness for all truncation angles was found to be steady with a single vortex-cell for small and moderate Grashof numbers. Small unstable vortex-cells were found inside the main vortex-cell that filled the gap cavity for high Grashof numbers (larger than $10^{6}$ ). As for the uniform gap cases, changes in gap ratio have a smaller effect on Nusselt number 
for a truncation angle of $90^{\circ}$ compared to the other truncation angles. This effect is shown in Figures 4.42 and 4.43.

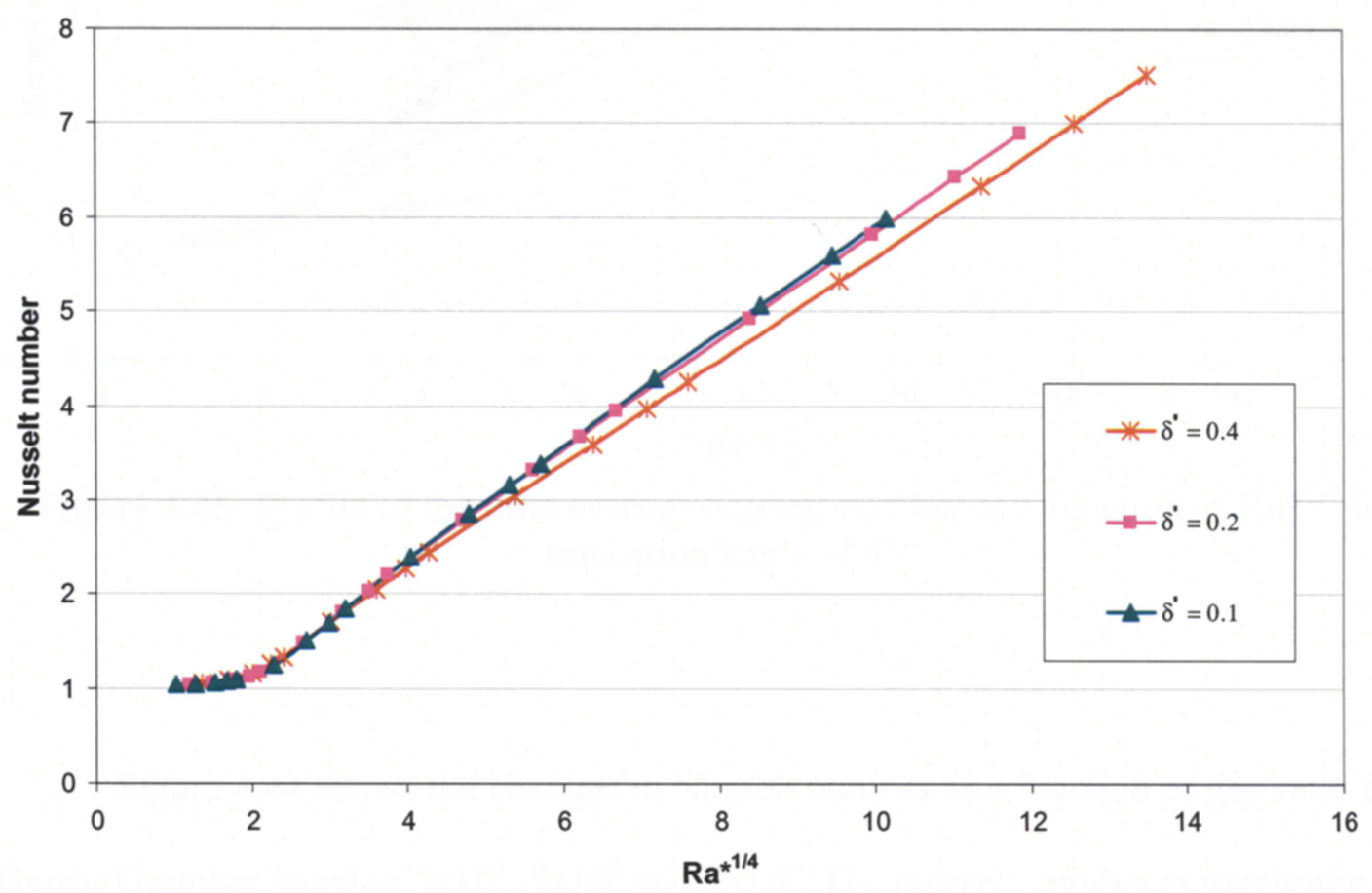

Figure 4.44: Profile of the time averaged Nusselt number as a function of $\mathrm{Ra}^{* 1 / 4}$ for a truncation angle of $90^{\circ}$ 


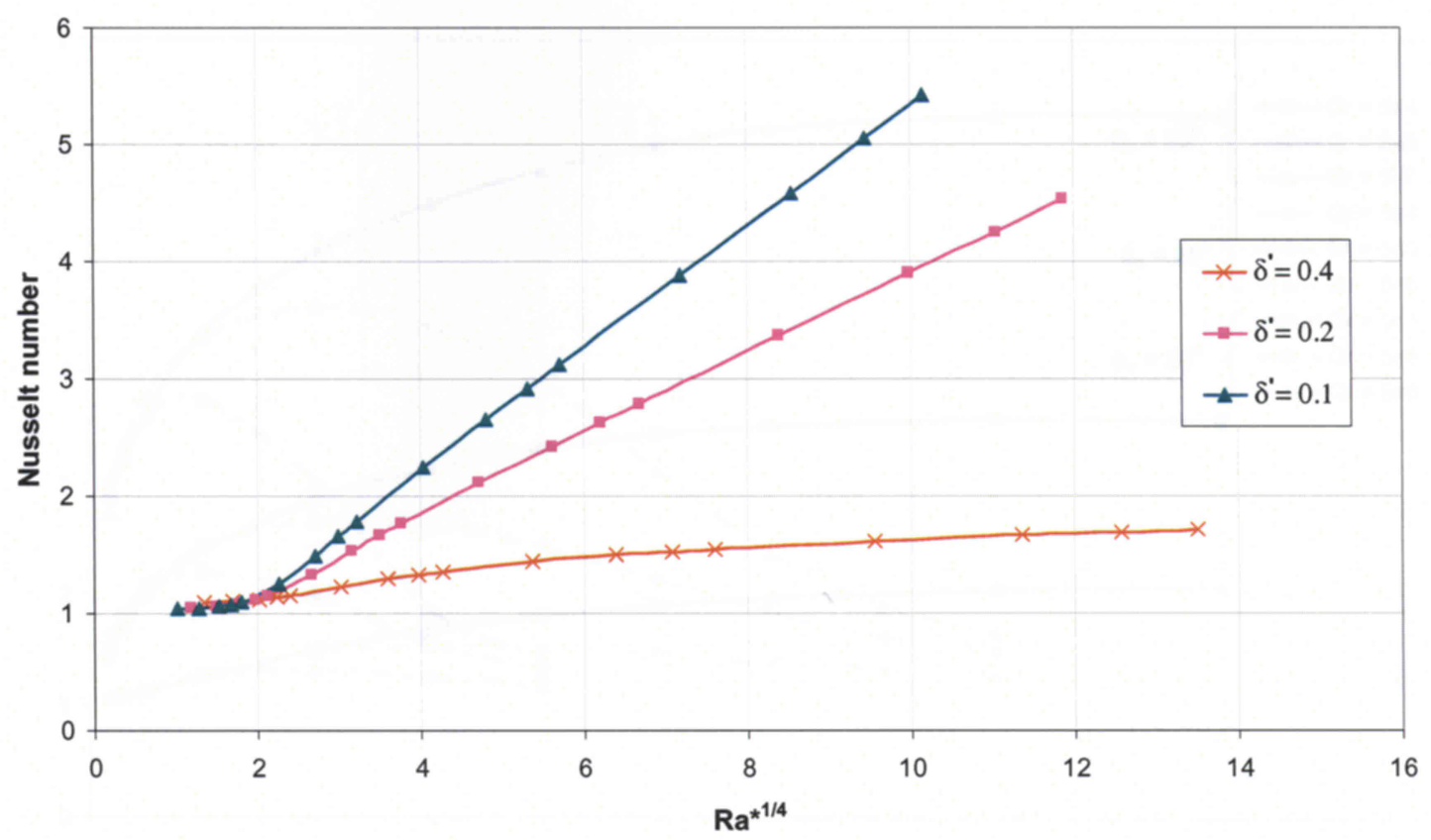

Figure 4.45: Profile of the time average Nusselt number as a function of $\mathrm{Ra}^{* 1 / 4}$ for a truncation angle of $45^{\circ}$

Figure 4.44 shows the changes in Nusselt number as a function of gap ratio for Grashof number equal to $5 \times 10^{4}, 5 \times 10^{5}$ and $5 \times 10^{6}$. The Nusselt number is maximum when $\delta$ ' is about $0.4,0.1$ and 0.05 for the truncation angles of $90^{\circ}, 45^{\circ}$ and $30^{\circ}$, respectively. 


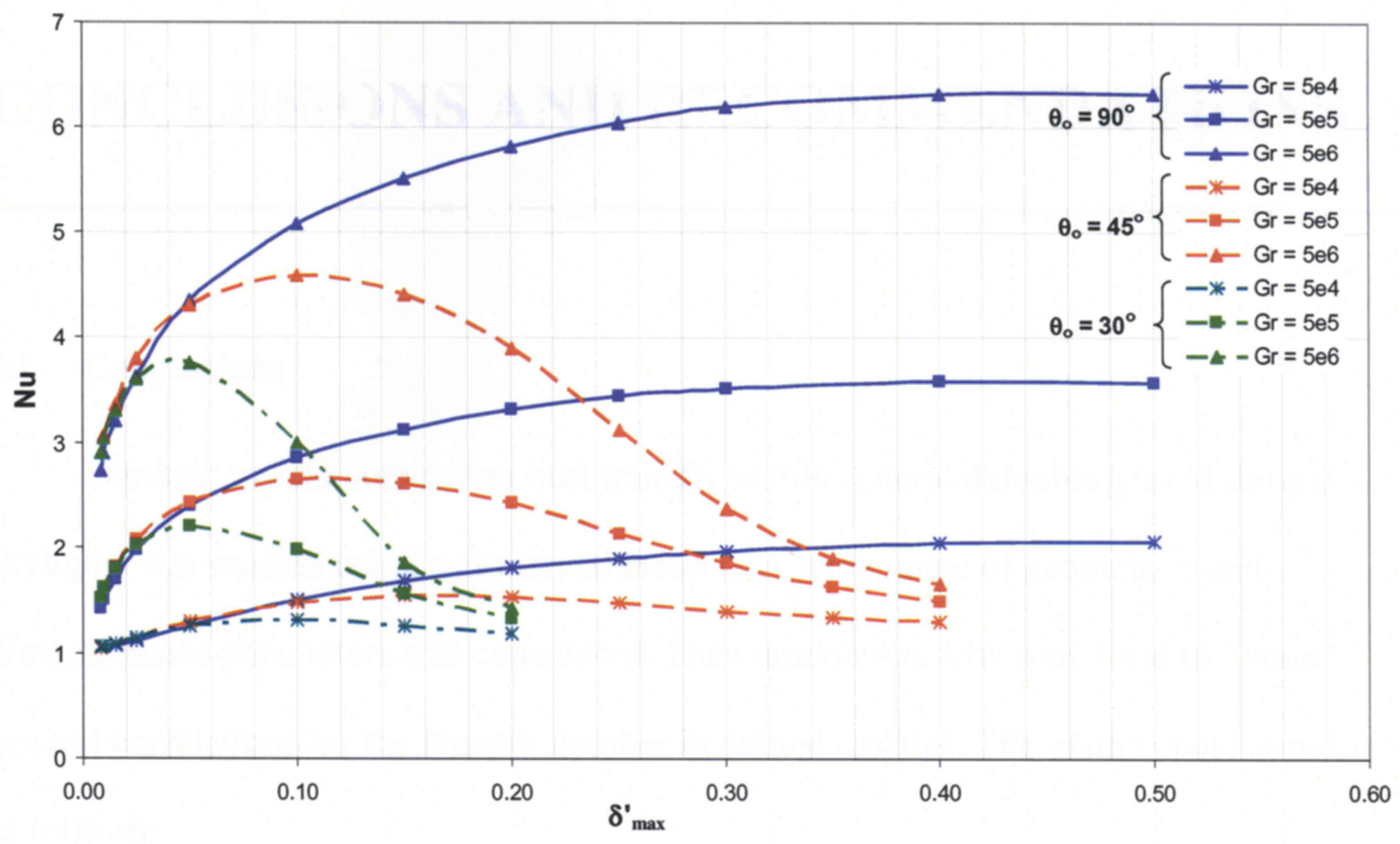

Figure 4.46: Nusselt number as a function of $\delta_{\text {max }}^{\prime}$ 


\section{CHAPTER 5}

\section{CONCLUSIONS AND RECOMMENDATIONS}

\subsection{Conclusions}

Laminar natural convection heat transfer within spherical double-glazed domed skylights was studied using a numerical method. A wide range of geometries and dimensionless parameters was considered. The numerical results were used to obtain general correlations for the Nusselt number in domed cavities. The main conclusions are as follows:

- Concentric domed cavities when heated from the interior surface:

The flow within a domed cavity for small and moderate Grashof numbers $\left(\mathrm{Gr}<10^{5}\right)$ might reach steady state conditions with a single vortex-cell or multi-vortex-cells, depending on the truncation angle and gap ratio. For high Grashof numbers $\left(\mathrm{Gr}>10^{5}\right)$ and a small gap ratio, the flow might be unsteady. In most of the cases the unsteady flow is periodic and oscillations in Nusselt number were observed.

The numerical results show that changes in the time-averaged Nusselt number as a function of $\mathrm{Ra}^{* 1 / 4}$ depend on the non-dimensional gap spacing $\delta$ for each 
truncation angle. For truncation angles of $45^{\circ}$ and $30^{\circ}$, this dependence is more significant.

In small gap cavities $(\delta \leq 0.2)$ minor oscillations in the time-averaged Nusselt number are also observed. These oscillations are caused by the changes in the flow pattern (Figure 4.12).

For a given cavity shape, there exists a critical gap spacing for which the Nusselt number reaches its maximum value and then decreases to settle at an asymptotic value with increasing gap spacing. The critical gap spacing was found to vary between $\delta=0.1$ and 0.3 , depending on the cavity shape.

Two different numerical solutions were found in some cases for moderate gap-to-radius ratio cavities $(0.5<\delta<0.2)$. Both solutions reach steady state conditions, one with a single-vortex-cell and the other with a bi-vortex-cell. The Nusselt number was found to be lower by $\sim 6 \%$ for the single-vortex-cell solution.

- Eccentric domed cavities when heated from the outsider-surface:

The numerical results reached steady state conditions with a single vortex-cell for small and moderate Grashof numbers $\left(\mathrm{Gr}<10^{5}\right)$. For high Grashof numbers $\left(\mathrm{Gr}>10^{5}\right)$, small unstable vortex-cells formed inside the big vortex cell. The unstable vortex-cells caused small oscillations in the Nusselt number. 
The vortex cells become stronger and bigger as the Grashof number increases. Depending on the truncation angle and gap thickness, at a Grashof number around $7.5 \times 10^{6}$, the flow might be unstable and gets into the transition condition, approaching the turbulent regime.

Similar to concentric domed cavities, changes in the gap ratio have a small effect on the Nusselt number for a truncation angle of $90^{\circ}$. However, the gap ratio has a significant effect on the Nusselt number for truncation angles of $45^{\circ}$ and $30^{\circ}$.

The Nusselt number is highest for a truncation angle of $90^{\circ}$ and increases with dimensionless gap thickness to an asymptotic value. For truncation angles of $45^{\circ}$ and $30^{\circ}$, the Nusselt number increases with the dimensionless gap thickness, reaching a maximum value and then decreasing. The critical gap thickness that maximizes the Nusselt number varies with the truncation angle and Grashof number.

\subsection{Recommendations}

The following recommendations are made for future studies:

Future studies should focus on heat transfer for the gap ratios of $\delta=0.35,0.25,0.015$, 0.008 and 0.005 , which need to be studied for the case of cavities heated from inside. 
Increasing the number of points will result in better a curve-fit and more accurate correlation.

Since there is no experimental result available for domed skylights, some experiments need to be conducted to validate the numerical results. These experiments should include flow visualization, in order to validate both the flow structures and the transition to unsteady flow.

In this study, in some cases, the numerical results converged to two different flow types. More scrutiny is therefore needed to study this flow bifurcation by numerical modelling and laboratory experiments. 


\section{APPENDIX-A}

\section{UNIFORM GAP THICKNESS DOMED}

\section{SKYLIGHT HEATED FROM INSIDE}

\section{A.1 Average Nusselt Number}

Table A.1: Average Nusselt number when $\theta_{0}=90^{\circ}$

\begin{tabular}{ccccc}
\hline $\mathbf{G r}$ & $\delta=\mathbf{1}$ & $\delta=\mathbf{0 . 8}$ & $\delta=\mathbf{0 . 5}$ & $\delta=\mathbf{0 . 4}$ \\
\hline $1.00 \mathrm{E}+03$ & 1.0907 & 1.0840 & 1.0634 & 1.0532 \\
$2.50 \mathrm{E}+03$ & 1.3677 & 1.3480 & 1.2718 & 1.2307 \\
$5.00 \mathrm{E}+03$ & 1.6915 & 1.6764 & 1.5748 & 1.5087 \\
$7.50 \mathrm{E}+03$ & 1.8997 & 1.8916 & 1.7881 & 1.7134 \\
$1.00 \mathrm{E}+04$ & 2.0548 & 2.0524 & 1.9506 & 1.8717 \\
$2.50 \mathrm{E}+04$ & 2.5990 & 2.6184 & 2.5306 & 2.4434 \\
$5.00 \mathrm{E}+04$ & 3.0681 & 3.1054 & 3.0320 & 2.9415 \\
$7.50 \mathrm{E}+04$ & 3.3676 & 3.4152 & 3.3500 & 3.2563 \\
$1.00 \mathrm{E}+05$ & 3.5943 & 3.6495 & 3.5900 & 3.4936 \\
$2.50 \mathrm{E}+05$ & 4.4365 & 4.5185 & 4.4755 & 4.3664 \\
$5.00 \mathrm{E}+05$ & 5.2421 & 5.3441 & 5.3046 & 5.1791 \\
$7.50 \mathrm{E}+05$ & 5.7926 & 5.9061 & 5.8632 & 5.7268 \\
$1.00 \mathrm{E}+06$ & 6.2214 & 6.3430 & 6.2953 & 6.1510 \\
$2.50 \mathrm{E}+06$ & 7.8194 & 7.9737 & 7.9162 & 7.7319 \\
$5.00 \mathrm{E}+06$ & 9.3032 & 9.4965 & 9.4353 & 9.0960 \\
$7.50 \mathrm{E}+06$ & 10.2966 & 10.5161 & 10.2969 & 10.0676 \\
$1.00 \mathrm{E}+07$ & 11.0627 & 11.3017 & 11.0658 & 10.8183 \\
\hline & & & & \\
\hline & & & & \\
\hline
\end{tabular}


Table A.1: Continued

\begin{tabular}{ccccc}
\hline $\mathbf{G r}$ & $\boldsymbol{\delta}=\mathbf{0 . 3}$ & $\boldsymbol{\delta}=\mathbf{0 . 2}$ & $\boldsymbol{\delta}=\mathbf{0 . 1}$ & $\delta=\mathbf{0 . 0 1}$ \\
\hline $1.00 \mathrm{E}+03$ & 1.0416 & 1.0281 & 1.0140 & 1.0008 \\
\hline $2.50 \mathrm{E}+03$ & 1.1812 & 1.1228 & 1.0606 & 1.0042 \\
\hline $5.00 \mathrm{E}+03$ & 1.4205 & 1.4324 & 1.2326 & 1.0119 \\
\hline $7.50 \mathrm{E}+03$ & 1.6093 & 1.6897 & 1.4671 & 1.0199 \\
$1.00 \mathrm{E}+04$ & 1.7584 & 1.8765 & 1.6825 & 1.0281 \\
\hline $2.50 \mathrm{E}+04$ & 2.3072 & 2.4720 & 2.3021 & 1.0907 \\
\hline $5.00 \mathrm{E}+04$ & 2.7926 & 2.5515 & 2.6784 & 1.2990 \\
\hline $7.50 \mathrm{E}+04$ & 3.1023 & 2.8464 & 2.7610 & 1.4553 \\
\hline $1.00 \mathrm{E}+05$ & 3.3322 & 3.0649 & 2.9469 & 1.5472 \\
\hline $2.50 \mathrm{E}+05$ & 4.1702 & 3.8401 & 3.7840 & 1.8637 \\
\hline $5.00 \mathrm{E}+05$ & 4.9530 & 4.5572 & 4.4808 & 2.2288 \\
\hline $7.50 \mathrm{E}+05$ & 5.4832 & 5.7545 & 4.9562 & 2.4723 \\
\hline $1.00 \mathrm{E}+06$ & 5.8799 & 6.1575 & 5.4214 & 2.6673 \\
\hline $2.50 \mathrm{E}+06$ & 7.5507 & 7.1514 & 6.4688 & 3.4020 \\
\hline $5.00 \mathrm{E}+06$ & 8.9363 & 8.9392 & 7.5623 & 4.0847 \\
\hline $7.50 \mathrm{E}+06$ & 9.8733 & 9.8676 & 8.2999 & 4.4699 \\
\hline $1.00 \mathrm{E}+07$ & 10.5877 & 10.5154 & 8.9080 & 4.7901 \\
\hline & & & & \\
\hline
\end{tabular}


Table A.2: Average Nusselt number when $\theta_{0}=45^{\circ}$

\begin{tabular}{ccccc}
\hline $\mathbf{G r}$ & $\delta=\mathbf{1}$ & $\delta=\mathbf{0 . 8}$ & $\delta=\mathbf{0 . 5}$ & $\delta=\mathbf{0 . 4}$ \\
\hline $1.00 \mathrm{E}+03$ & 1.0303 & 1.0438 & 1.0528 & 1.0494 \\
$2.50 \mathrm{E}+03$ & 1.2425 & 1.3074 & 1.3405 & 1.3106 \\
\hline $5.00 \mathrm{E}+03$ & 1.5704 & 1.6700 & 1.7396 & 1.7022 \\
$7.50 \mathrm{E}+03$ & 1.7833 & 1.8980 & 1.9885 & 1.9548 \\
$1.00 \mathrm{E}+04$ & 1.9380 & 2.0627 & 2.1684 & 2.1379 \\
$2.50 \mathrm{E}+04$ & 2.4641 & 2.6212 & 2.7785 & 2.7582 \\
\hline $5.00 \mathrm{E}+04$ & 2.9326 & 3.1058 & 3.3015 & 3.2867 \\
$7.50 \mathrm{E}+04$ & 3.2301 & 3.4243 & 3.6403 & 3.6272 \\
$1.00 \mathrm{E}+05$ & 3.4665 & 3.6694 & 3.8981 & 3.8856 \\
\hline $2.50 \mathrm{E}+05$ & 4.3540 & 4.5826 & 4.8400 & 4.8313 \\
\hline $5.00 \mathrm{E}+05$ & 5.1962 & 5.4426 & 5.7168 & 5.7075 \\
$7.50 \mathrm{E}+05$ & 5.7715 & 6.0282 & 6.3106 & 6.2995 \\
$1.00 \mathrm{E}+06$ & 6.2211 & 6.4869 & 6.7758 & 6.7745 \\
\hline $2.50 \mathrm{E}+06$ & 7.9392 & 8.2573 & 8.5590 & 8.5620 \\
\hline $5.00 \mathrm{E}+06$ & 9.5458 & 9.8950 & 10.2328 & 10.2049 \\
$7.50 \mathrm{E}+06$ & 10.6143 & 10.9851 & 11.3537 & 11.2950 \\
$1.00 \mathrm{E}+07$ & 11.4369 & 11.8246 & 12.2158 & 12.1297 \\
\hline & & & & \\
\hline & & & & \\
\hline
\end{tabular}


Table A.2: Continued

\begin{tabular}{|c|c|c|c|c|c|}
\hline Gr & $\delta=0.3$ & $\delta=0.2$ & $\delta=0.1$ & Gr & $\delta=\mathbf{0 . 0 1}$ \\
\hline $1.00 \mathrm{E}+03$ & 1.0423 & 1.0327 & 1.0184 & $1.00 \mathrm{E}+03$ & 1.0009 \\
\hline $2.50 \mathrm{E}+03$ & 1.2602 & 2.4727 & 1.0989 & $2.50 \mathrm{E}+03$ & 1.0051 \\
\hline $5.00 \mathrm{E}+03$ & 1.7559 & 3.5869 & 1.5659 & $5.00 \mathrm{E}+03$ & 1.0172 \\
\hline $7.50 \mathrm{E}+03$ & 2.0467 & 4.2577 & 2.0105 & $7.50 \mathrm{E}+03$ & 1.0313 \\
\hline $1.00 \mathrm{E}+04$ & 2.2479 & 4.6940 & 2.2348 & $8.50 \mathrm{E}+03$ & 1.0371 \\
\hline $2.50 \mathrm{E}+04$ & 2.9125 & 6.0850 & 2.9266 & $1.00 \mathrm{E}+04$ & 1.0456 \\
\hline $5.00 \mathrm{E}+04$ & 3.4787 & 7.1885 & 3.5024 & $2.00 \mathrm{E}+04$ & 1.1010 \\
\hline $7.50 \mathrm{E}+04$ & 3.8410 & 7.5849 & 3.8749 & $3.00 \mathrm{E}+04$ & 1.1688 \\
\hline $1.00 \mathrm{E}+05$ & 4.1121 & 8.1035 & 4.1505 & $4.00 \mathrm{E}+04$ & 1.2581 \\
\hline $2.50 \mathrm{E}+05$ & 5.0449 & 10.0052 & 5.0352 & $5.00 \mathrm{E}+04$ & 1.3497 \\
\hline $5.00 \mathrm{E}+05$ & 5.6078 & 11.7752 & 5.9081 & $6.00 \mathrm{E}+04$ & 1.4648 \\
\hline $7.50 \mathrm{E}+05$ & 6.1987 & 12.9189 & 6.4603 & $7.00 \mathrm{E}+04$ & 1.6019 \\
\hline $1.00 \mathrm{E}+06$ & 6.6655 & 13.8508 & 6.8635 & $8.00 \mathrm{E}+04$ & 1.6828 \\
\hline $2.50 \mathrm{E}+06$ & 8.4066 & 16.7966 & 8.3413 & $9.00 \mathrm{E}+04$ & 1.7429 \\
\hline $5.00 \mathrm{E}+06$ & 9.9787 & 19.3743 & 9.6822 & $1.00 \mathrm{E}+05$ & 1.7939 \\
\hline $7.50 \mathrm{E}+06$ & 11.0876 & 21.3264 & 10.4874 & $2.00 \mathrm{E}+05$ & 2.0603 \\
\hline \multirow[t]{11}{*}{$1.00 \mathrm{E}+07$} & 11.9219 & 22.8584 & 11.1000 & $4.00 \mathrm{E}+05$ & 2.2946 \\
\hline & & & & $5.00 \mathrm{E}+05$ & 2.4312 \\
\hline & & & & $6.00 \mathrm{E}+05$ & 2.6203 \\
\hline & & & & $8.00 \mathrm{E}+05$ & 2.8233 \\
\hline & & & & $1.00 \mathrm{E}+06$ & 2.9606 \\
\hline & & & & $2.00 \mathrm{E}+06$ & 3.4734 \\
\hline & & & & $4.00 \mathrm{E}+06$ & 4.0711 \\
\hline & & & & $5.00 \mathrm{E}+06$ & 4.2663 \\
\hline & & & & $6.00 \mathrm{E}+06$ & 4.5132 \\
\hline & & & & $8.00 \mathrm{E}+06$ & 4.7299 \\
\hline & & & & $1.00 \mathrm{E}+07$ & 4.9946 \\
\hline
\end{tabular}


Table A.3: Average Nusselt number when $\theta_{0}=30^{\circ}$

\begin{tabular}{ccccc}
\hline $\mathbf{G r}$ & $\delta=\mathbf{1}$ & $\delta=\mathbf{0 . 8}$ & $\delta=\mathbf{0 . 5}$ & $\delta=\mathbf{0 . 4}$ \\
\hline $1.00 \mathrm{E}+03$ & 1.0024 & 1.0065 & 1.0235 & 1.0286 \\
$2.50 \mathrm{E}+03$ & 1.0328 & 1.0924 & 1.2574 & 1.2880 \\
$5.00 \mathrm{E}+03$ & 1.2096 & 1.3773 & 1.6558 & 1.7087 \\
$7.50 \mathrm{E}+03$ & 1.4005 & 1.5988 & 1.9055 & 1.9669 \\
$1.00 \mathrm{E}+04$ & 1.5553 & 1.7629 & 2.0816 & 2.1495 \\
$2.50 \mathrm{E}+04$ & 2.1005 & 2.3077 & 2.6637 & 2.7557 \\
$5.00 \mathrm{E}+04$ & 2.5686 & 2.7720 & 3.1614 & 3.2736 \\
$7.50 \mathrm{E}+04$ & 2.8752 & 3.0801 & 3.4876 & 3.6116 \\
$1.00 \mathrm{E}+05$ & 3.1117 & 3.3199 & 3.7381 & 3.8697 \\
$2.50 \mathrm{E}+05$ & 4.0039 & 4.2343 & 4.6712 & 4.8176 \\
$5.00 \mathrm{E}+05$ & 4.8677 & 5.1196 & 5.5513 & 5.6966 \\
$7.50 \mathrm{E}+05$ & 5.4668 & 5.7300 & 6.1511 & 6.2925 \\
$1.00 \mathrm{E}+06$ & 5.9378 & 6.2095 & 6.6194 & 6.7552 \\
$2.50 \mathrm{E}+06$ & 7.7161 & 8.0101 & 8.3747 & 8.4803 \\
$5.00 \mathrm{E}+06$ & 9.3742 & 9.6795 & 10.0223 & 10.0768 \\
$7.50 \mathrm{E}+06$ & 10.4014 & 10.7981 & 11.1456 & 11.1577 \\
$1.00 \mathrm{E}+07$ & 11.2455 & 11.6586 & 12.0204 & 12.0075 \\
\hline & & & & \\
\hline & & & & \\
\hline
\end{tabular}


Table A.3: Continued

\begin{tabular}{ccccc}
\hline $\mathbf{G r}$ & $\delta=\mathbf{0 . 3}$ & $\delta=\mathbf{0 . 2}$ & $\delta=\mathbf{0 . 1}$ & $\delta=\mathbf{0 . 0 1}$ \\
\hline $1.00 \mathrm{E}+03$ & 1.0286 & 1.0239 & 1.0147 & 1.0011 \\
$2.50 \mathrm{E}+03$ & 1.2693 & 1.2270 & 1.1159 & 1.0070 \\
\hline $5.00 \mathrm{E}+03$ & 1.6949 & 1.7521 & 1.7585 & 1.0237 \\
\hline $7.50 \mathrm{E}+03$ & 1.9597 & 2.0509 & 2.0934 & 1.0419 \\
$1.00 \mathrm{E}+04$ & 2.1473 & 2.2552 & 2.3057 & 1.0990 \\
\hline $2.50 \mathrm{E}+04$ & 2.7714 & 2.9249 & 2.9720 & 1.8632 \\
\hline $5.00 \mathrm{E}+04$ & 3.3038 & 3.4948 & 3.5516 & 2.3307 \\
\hline $7.50 \mathrm{E}+04$ & 3.6499 & 3.8593 & 3.9204 & 2.5536 \\
\hline $1.00 \mathrm{E}+05$ & 3.9139 & 4.1302 & 4.1919 & 2.7082 \\
\hline $2.50 \mathrm{E}+05$ & 4.8779 & 4.7537 & 4.9496 & 3.1316 \\
\hline $5.00 \mathrm{E}+05$ & 5.7554 & 5.6294 & 5.7530 & 3.5821 \\
\hline $7.50 \mathrm{E}+05$ & 6.3416 & 6.2193 & 6.2345 & 3.7389 \\
\hline $1.00 \mathrm{E}+06$ & 6.7974 & 6.6801 & 6.6123 & 3.9949 \\
\hline $2.50 \mathrm{E}+06$ & 8.5318 & 8.4509 & 8.1011 & 4.6222 \\
\hline $5.00 \mathrm{E}+06$ & 10.1968 & 10.1070 & 9.4403 & 5.2407 \\
\hline $7.50 \mathrm{E}+06$ & 11.3254 & 11.1953 & 10.2779 & 5.5655 \\
\hline $1.00 \mathrm{E}+07$ & 12.2021 & 12.0257 & 10.9885 & 5.8540 \\
\hline
\end{tabular}




\section{A.2 Curve-Fitting to Coefficient $c$ and $d$}

Table A.4: Coefficient $c$ and $d$ for $\theta_{0}=90^{\circ}$

\begin{tabular}{ccccccc}
\hline$\delta$ & $\boldsymbol{c}$ & $\begin{array}{c}\boldsymbol{c} \\
\text { (Curve } \\
\text { Fitting) }\end{array}$ & $\begin{array}{c}\% \\
\text { Difference }\end{array}$ & $\boldsymbol{d}$ & $\begin{array}{c}\boldsymbol{d} \\
\text { (Curve } \\
\text { Fitting) }\end{array}$ & $\begin{array}{c}\% \\
\text { Difference }\end{array}$ \\
\hline 1 & 0.7801 & 0.7792 & $0.11 \%$ & 0.9914 & 0.9944 & $-0.31 \%$ \\
0.8 & 0.7721 & 0.7745 & $-0.31 \%$ & 1.0068 & 1.0011 & $0.56 \%$ \\
0.5 & 0.7622 & 0.7611 & $0.14 \%$ & 1.0258 & 1.0245 & $0.13 \%$ \\
0.4 & 0.7610 & 0.7537 & $0.96 \%$ & 1.0293 & 1.0376 & $-0.80 \%$ \\
0.3 & 0.7402 & 0.7493 & $-1.23 \%$ & 1.0513 & 1.0464 & $0.47 \%$ \\
0.2 & 0.8164 & 0.8151 & $0.16 \%$ & 1.0339 & 1.0359 & $-0.19 \%$ \\
0.1 & 0.8994 & 0.9002 & $-0.09 \%$ & 1.0097 & 1.0094 & $0.03 \%$ \\
0.01 & 0.8430 & 0.8430 & $0.00 \%$ & 0.9869 & 0.9869 & $0.00 \%$ \\
\hline
\end{tabular}

Table A.5: Coefficient $c$ and $d$ for $\theta_{0}=45^{\circ}$

\begin{tabular}{ccccccc}
\hline$\delta$ & $\boldsymbol{c}$ & $\begin{array}{c}c \\
\text { (Curve } \\
\text { Fitting) }\end{array}$ & $\begin{array}{c}\% \\
\text { Difference }\end{array}$ & $\boldsymbol{d}$ & $\begin{array}{c}\boldsymbol{d} \\
\text { (Curve } \\
\text { Fitting) }\end{array}$ & $\begin{array}{c}\% \\
\text { Difference }\end{array}$ \\
\hline 1 & 0.6992 & 0.6933 & $0.85 \%$ & 1.0435 & 1.0300 & $1.29 \%$ \\
0.8 & 0.7436 & 0.7542 & $-1.43 \%$ & 1.0373 & 1.0300 & $0.70 \%$ \\
0.5 & 0.8337 & 0.8234 & $1.24 \%$ & 1.0233 & 1.0300 & $-0.65 \%$ \\
0.4 & 0.8229 & 0.8295 & $-0.81 \%$ & 1.0447 & 1.0300 & $1.41 \%$ \\
0.3 & 0.9049 & 0.9179 & $-1.44 \%$ & 1.0239 & 1.0300 & $-0.60 \%$ \\
0.2 & 2.1270 & 2.1165 & $0.49 \%$ & 0.9809 & 0.9718 & $0.93 \%$ \\
0.1 & 1.1404 & 1.2192 & $-6.91 \%$ & 1.0233 & 0.9718 & $5.03 \%$ \\
0.01 & 0.9335 & 0.8705 & $6.75 \%$ & 0.9683 & 0.9718 & $-0.36 \%$ \\
\hline
\end{tabular}


Table A.6: Coefficient $c$ and $d$ for $\theta_{0}=30^{\circ}$

\begin{tabular}{ccccccc}
\hline$\delta$ & $\boldsymbol{c}$ & $\begin{array}{c}\boldsymbol{c} \\
\text { (Curve } \\
\text { Fitting) }\end{array}$ & $\begin{array}{c}\% \\
\text { Difference }\end{array}$ & $\boldsymbol{d}$ & $\begin{array}{c}\boldsymbol{d} \\
\text { (Curve } \\
\text { Fitting) }\end{array}$ & $\begin{array}{c}\% \\
\text { Difference }\end{array}$ \\
\hline 1 & 0.5173 & 0.5170 & $0.05 \%$ & 1.1585 & 1.1589 & $-0.04 \%$ \\
0.8 & 0.5956 & 0.5964 & $-0.14 \%$ & 1.1174 & 1.1157 & $0.15 \%$ \\
0.5 & 0.7650 & 0.7681 & $-0.41 \%$ & 1.0526 & 1.0556 & $-0.29 \%$ \\
0.4 & 0.8465 & 0.8266 & $2.35 \%$ & 1.0271 & 1.0363 & $-0.89 \%$ \\
0.3 & 0.8815 & 0.9255 & $-4.99 \%$ & 1.0373 & 1.0108 & $2.56 \%$ \\
0.2 & 1.1275 & 1.1019 & $2.27 \%$ & 0.9511 & 0.9721 & $-2.21 \%$ \\
0.1 & 1.3497 & 1.3591 & $-0.70 \%$ & 0.9166 & 0.9112 & $0.59 \%$ \\
0.01 & 1.4710 & 1.4693 & $0.12 \%$ & 0.8279 & 0.8278 & $0.01 \%$ \\
\hline
\end{tabular}




\section{A.3 Curve-Fitting to The Nusselt Number}

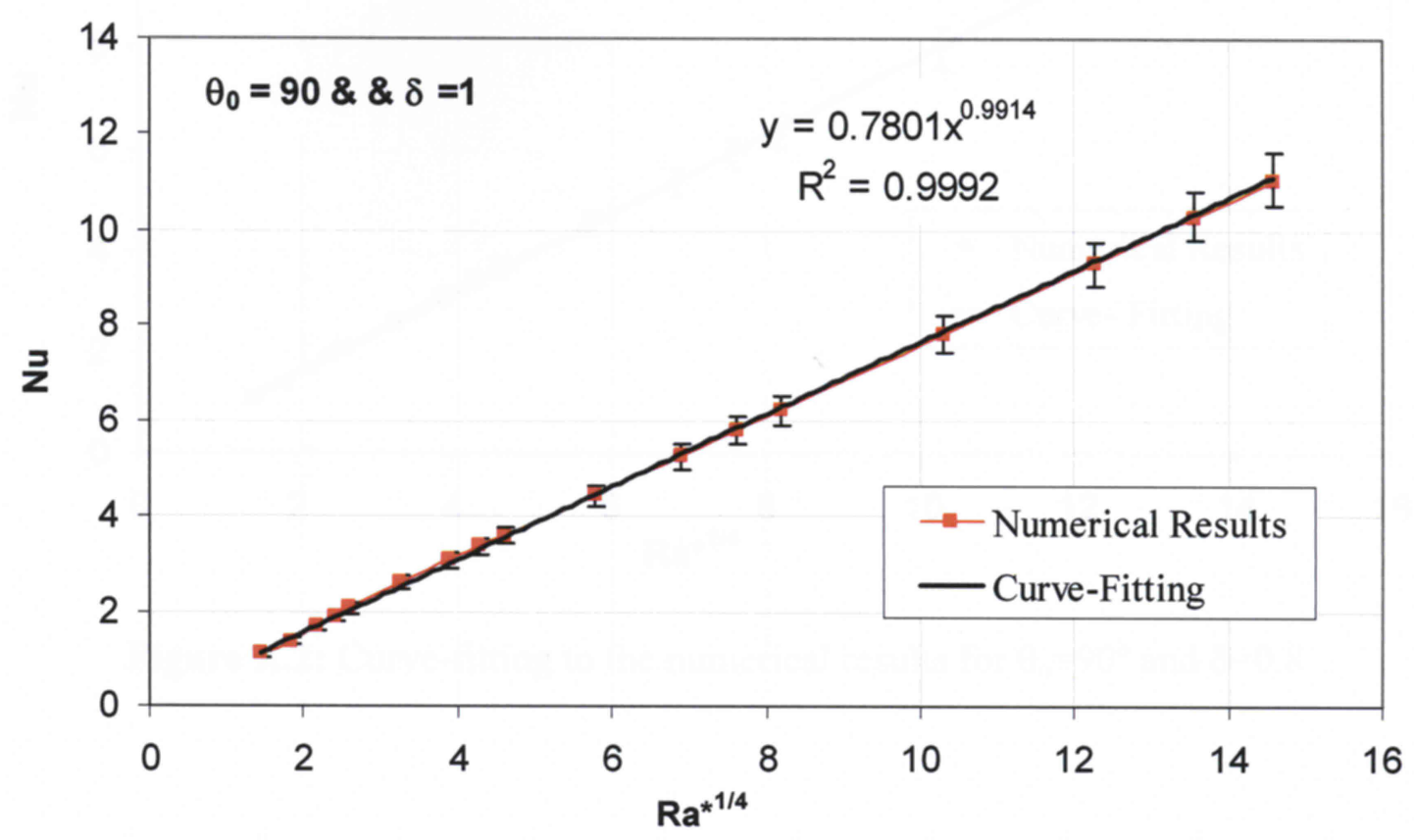

Figure A.1: Curve-fitting to the numerical results for $\theta_{0}=90^{\circ}$ and $\delta=1$ 


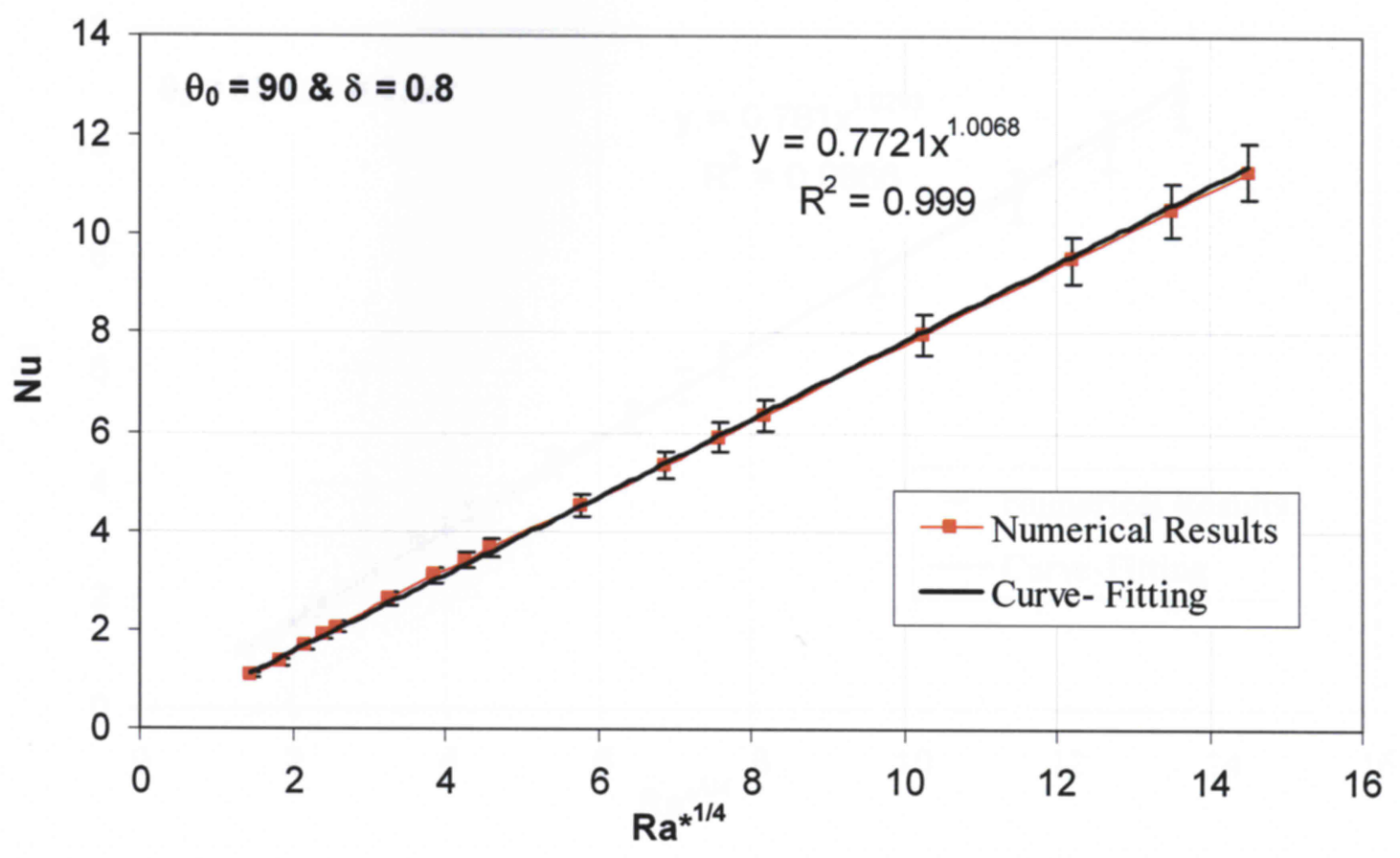

Figure A.2: Curve-fitting to the numerical results for $\theta_{0}=90^{\circ}$ and $\delta=0.8$

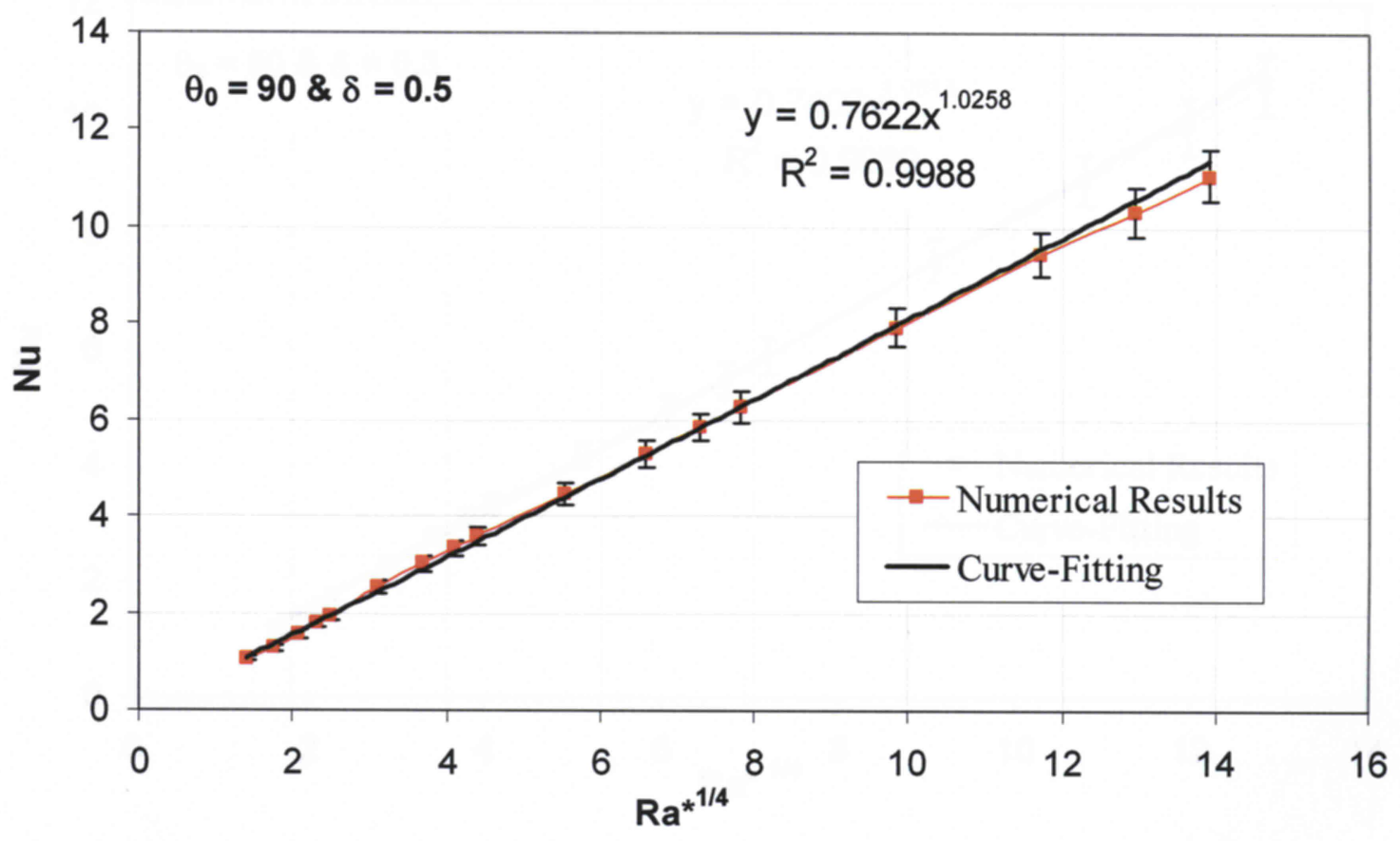

Figure A.3: Curve-fitting to the numerical results for $\theta_{0}=90^{\circ}$ and $\delta=0.5$ 


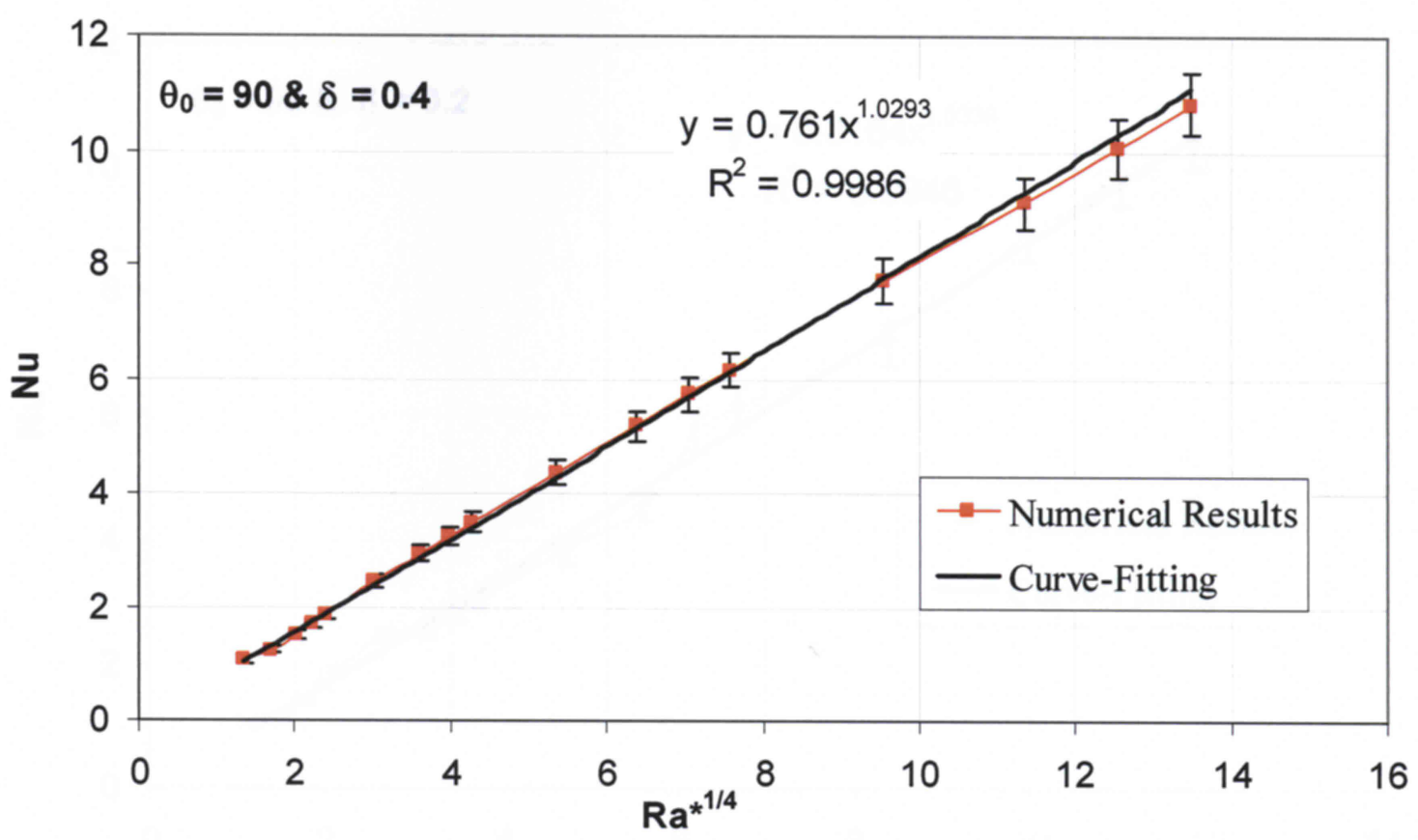

Figure A.4: Curve-fitting to the numerical results for $\theta_{0}=90^{\circ}$ and $\delta=0.4$

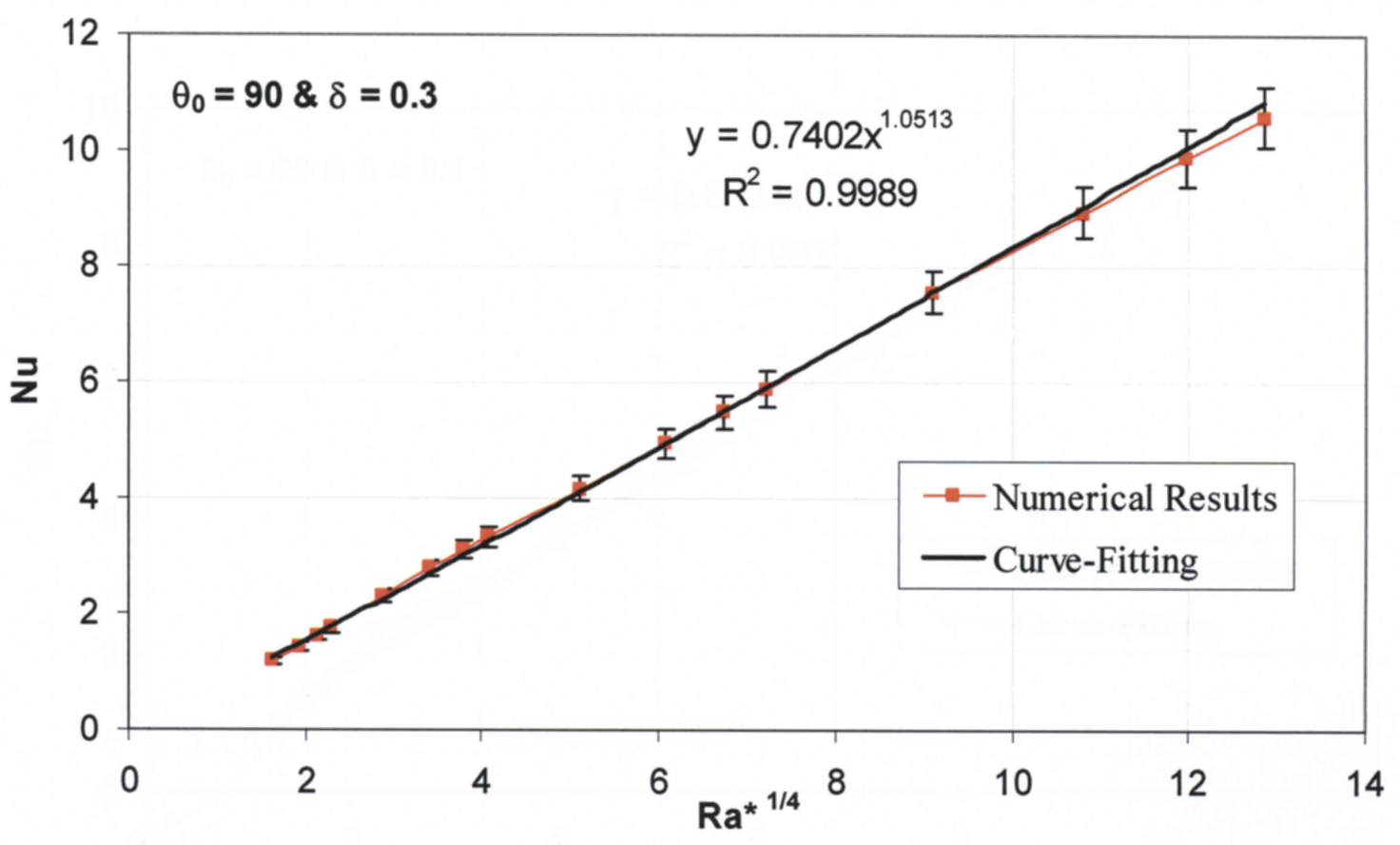

Figure A.5: Curve-fitting to the numerical results for $\theta_{0}=90^{\circ}$ and $\delta=0.3$ 


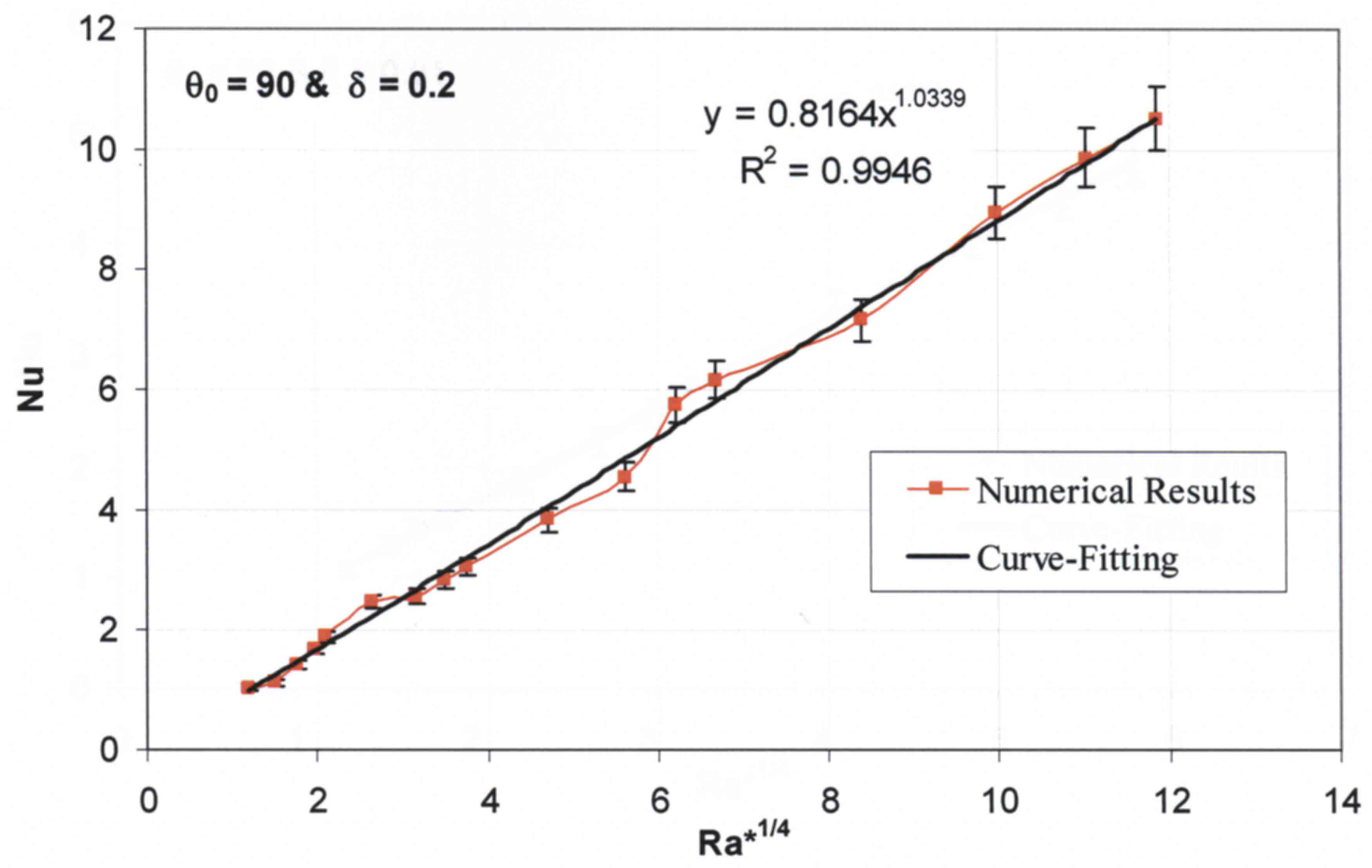

Figure A.6: Curve-fitting to the numerical results for $\theta_{0}=90^{\circ}$ and $\delta=0.2$

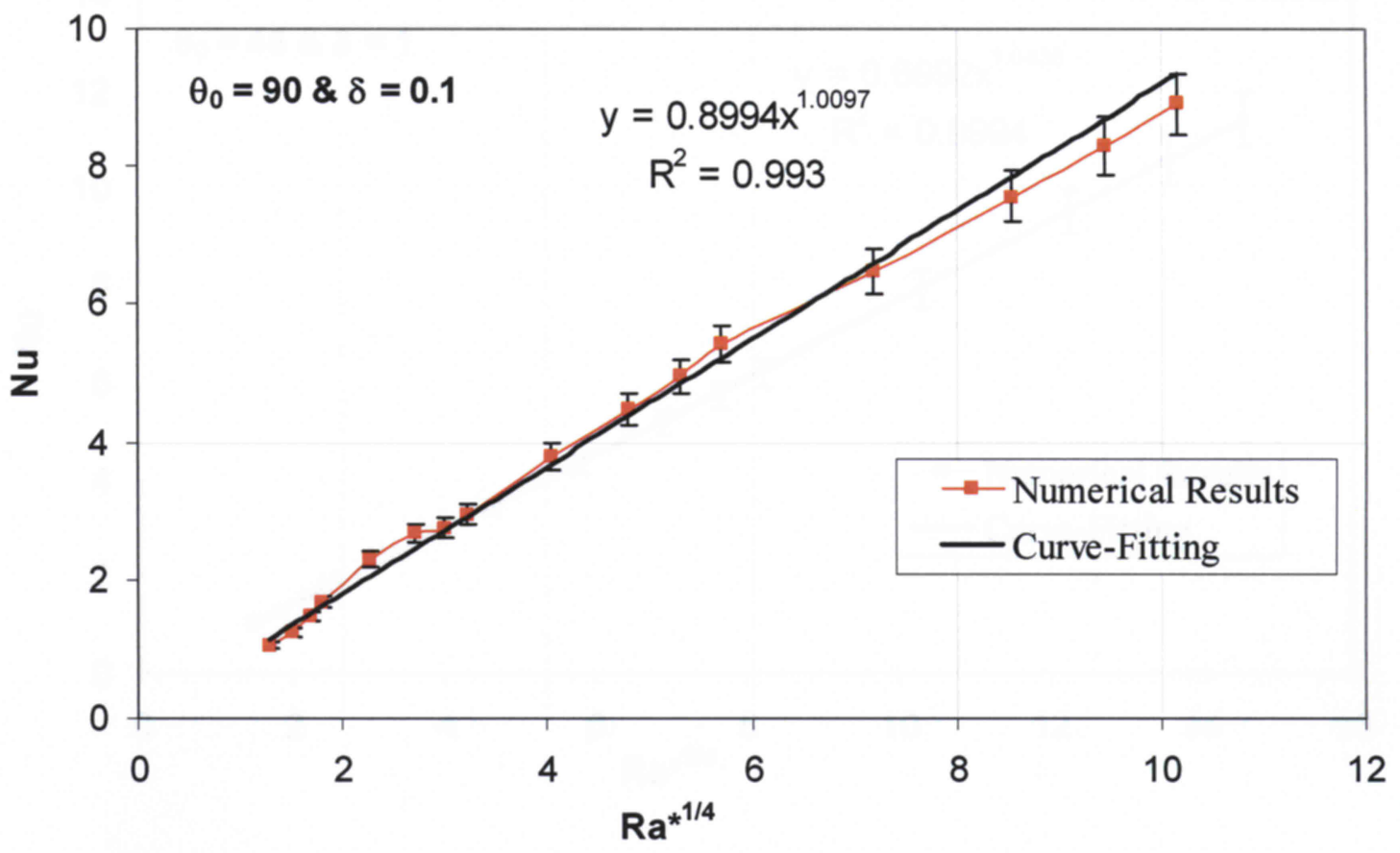

Figure A.7: Curve-fitting to the numerical results for $\theta_{0}=90^{\circ}$ and $\delta=0.1$ 


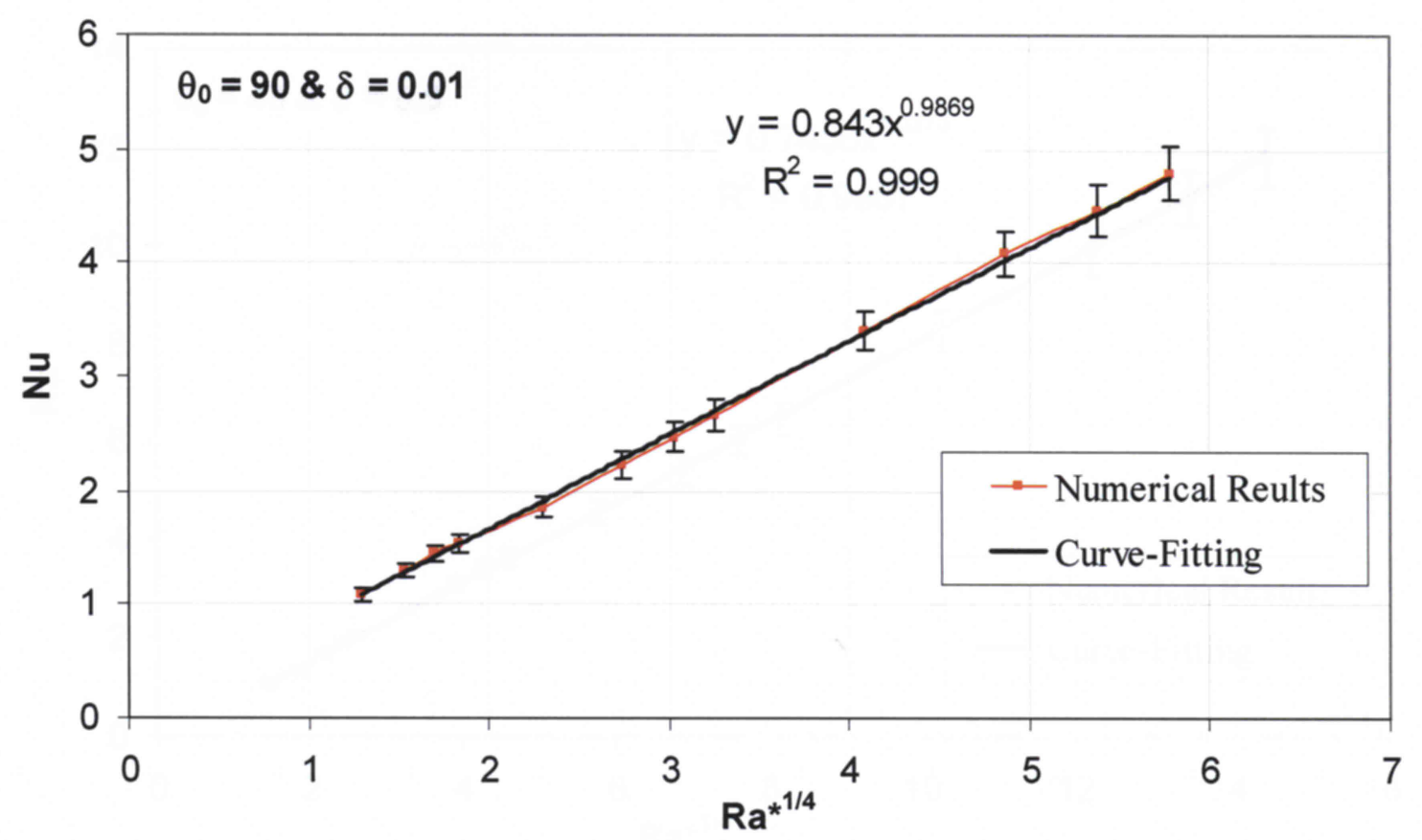

Figure A.8: Curve-fitting to the numerical results for $\theta_{0}=90^{\circ}$ and $\delta=0.01$

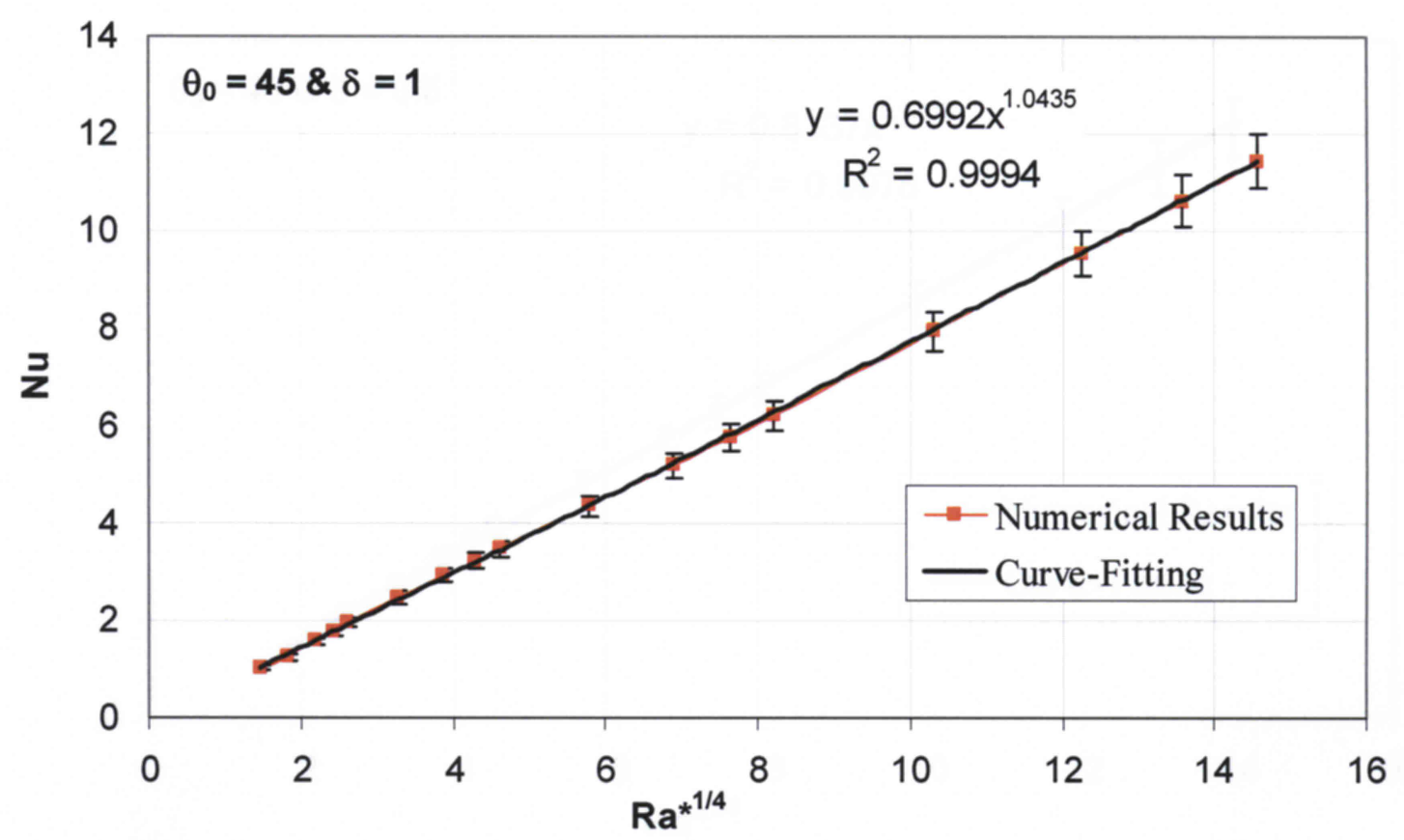

Figure A.9: Curve-fitting to the numerical results for $\theta_{0}=45^{\circ}$ and $\delta=1$ 




Figure A.10: Curve-fitting to the numerical results for $\theta_{0}=45^{\circ}$ and $\delta=0.8$

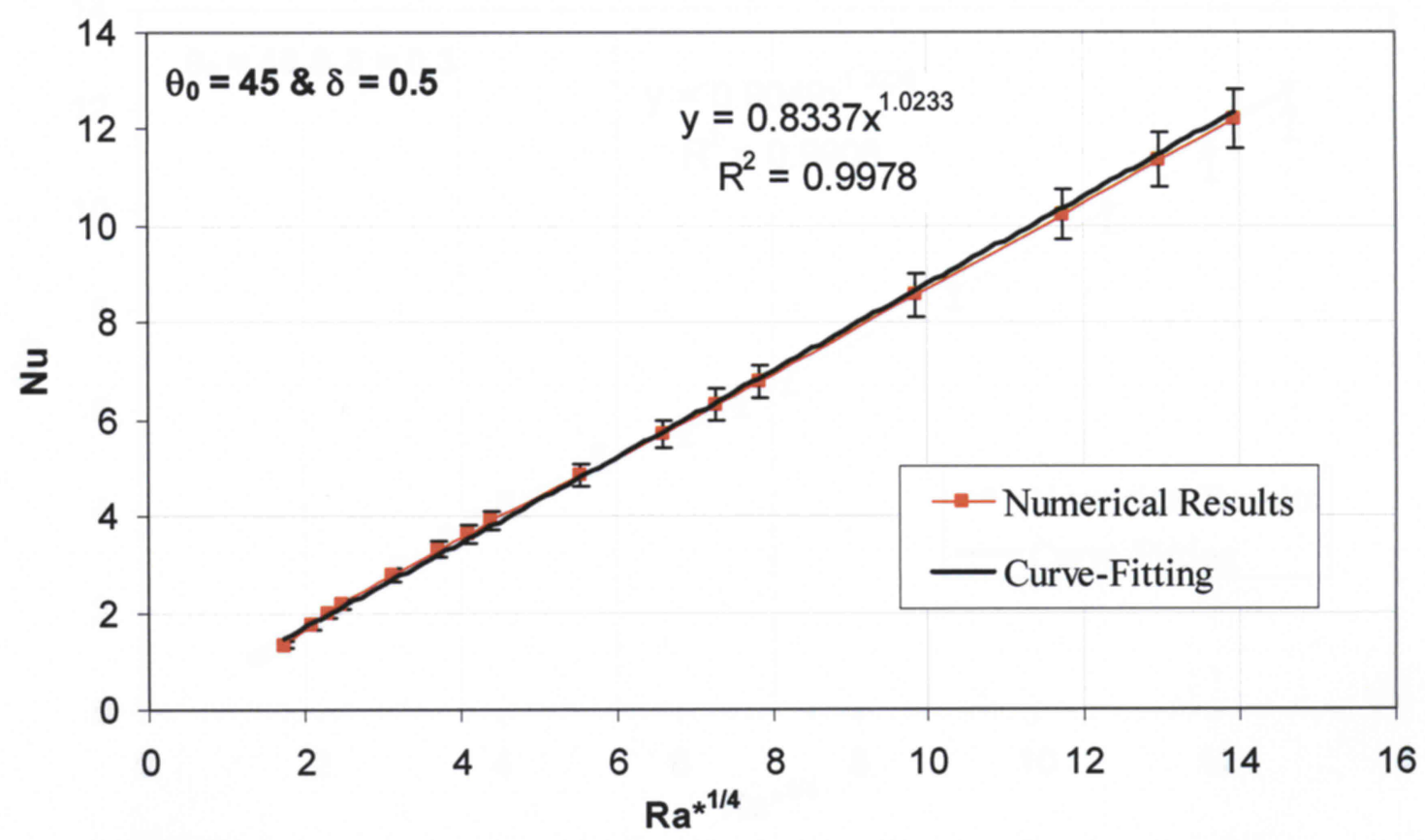

Figure A.11: Curve-fitting to the numerical results for $\theta_{0}=45^{\circ}$ and $\delta=0.5$ 


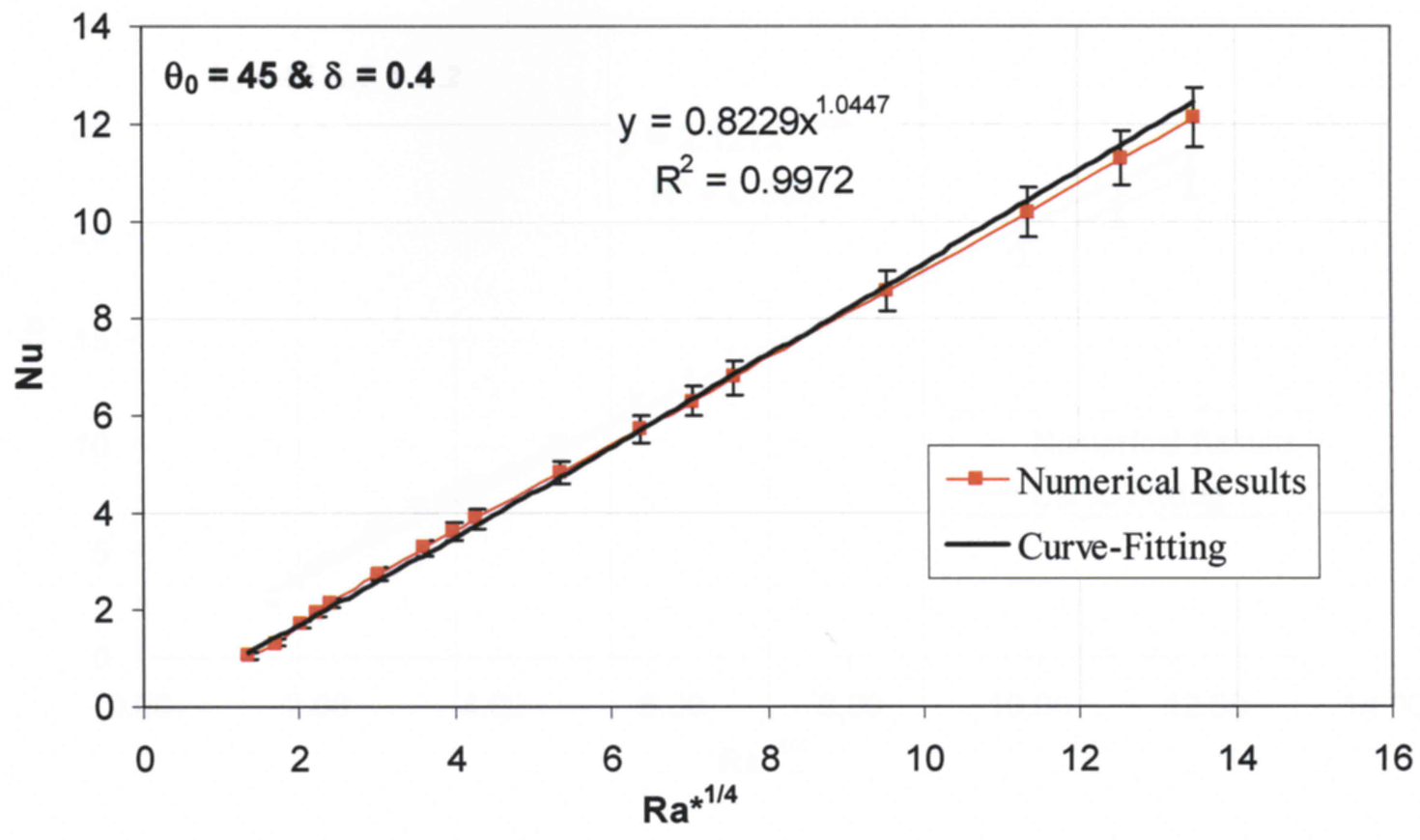

Figure A.12: Curve-fitting to the numerical results for $\theta_{0}=45^{\circ}$ and $\delta=0.4$

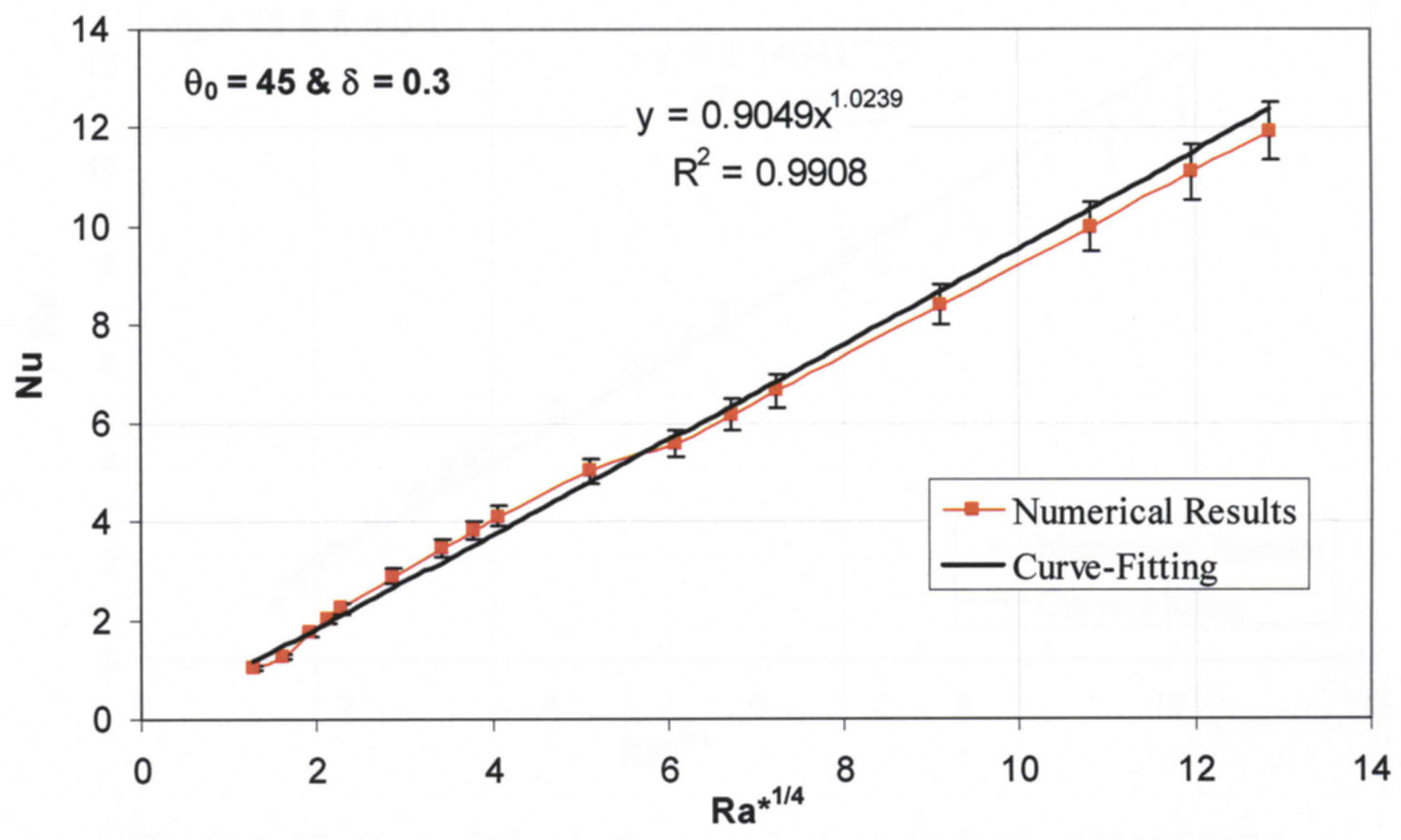

Figure A.13: Curve-fitting to the numerical results for $\theta_{0}=45^{\circ}$ and $\delta=0.3$ 


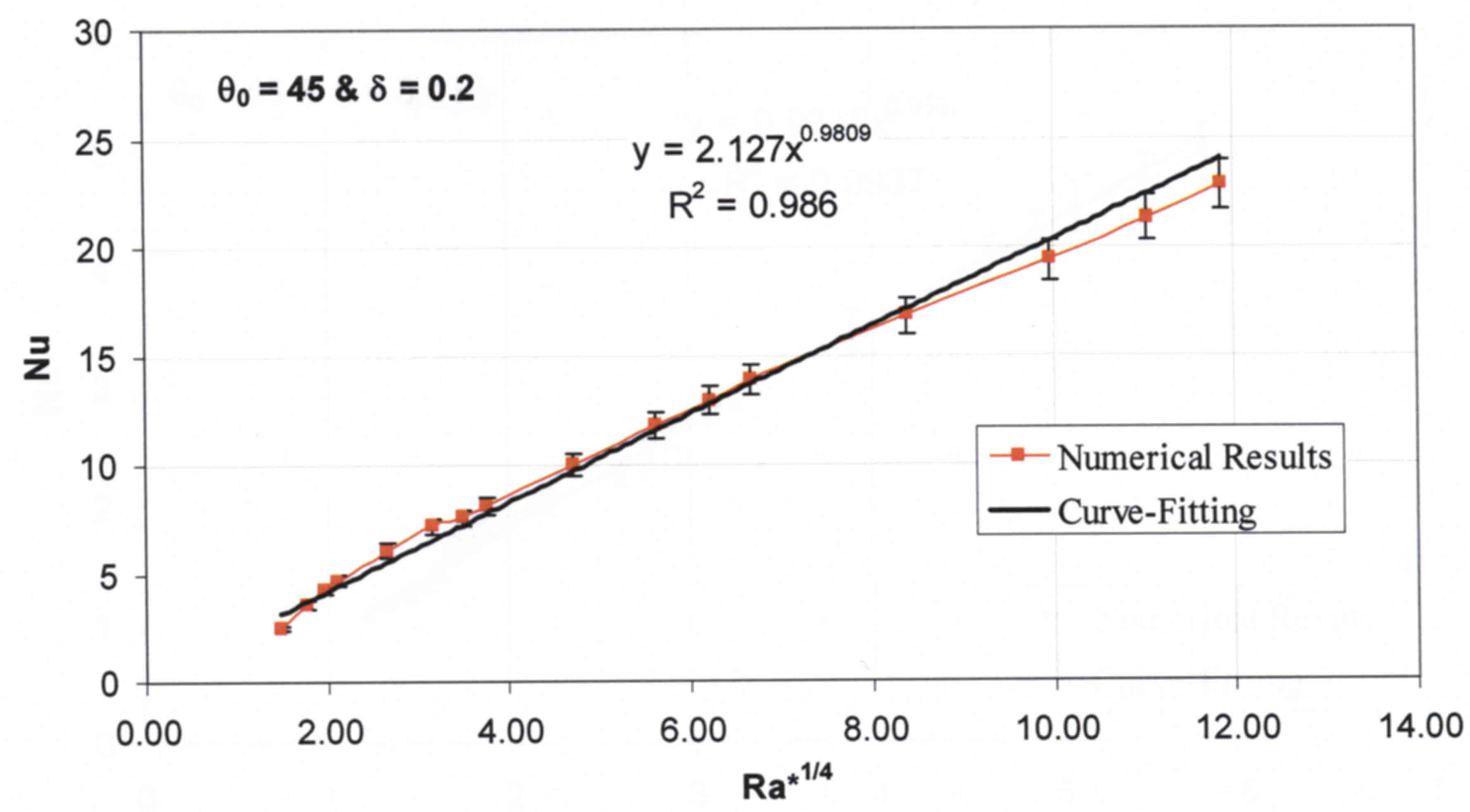

Figure A.14: Curve-fitting to the numerical results for $\theta_{0}=45^{\circ}$ and $\delta=0.2$

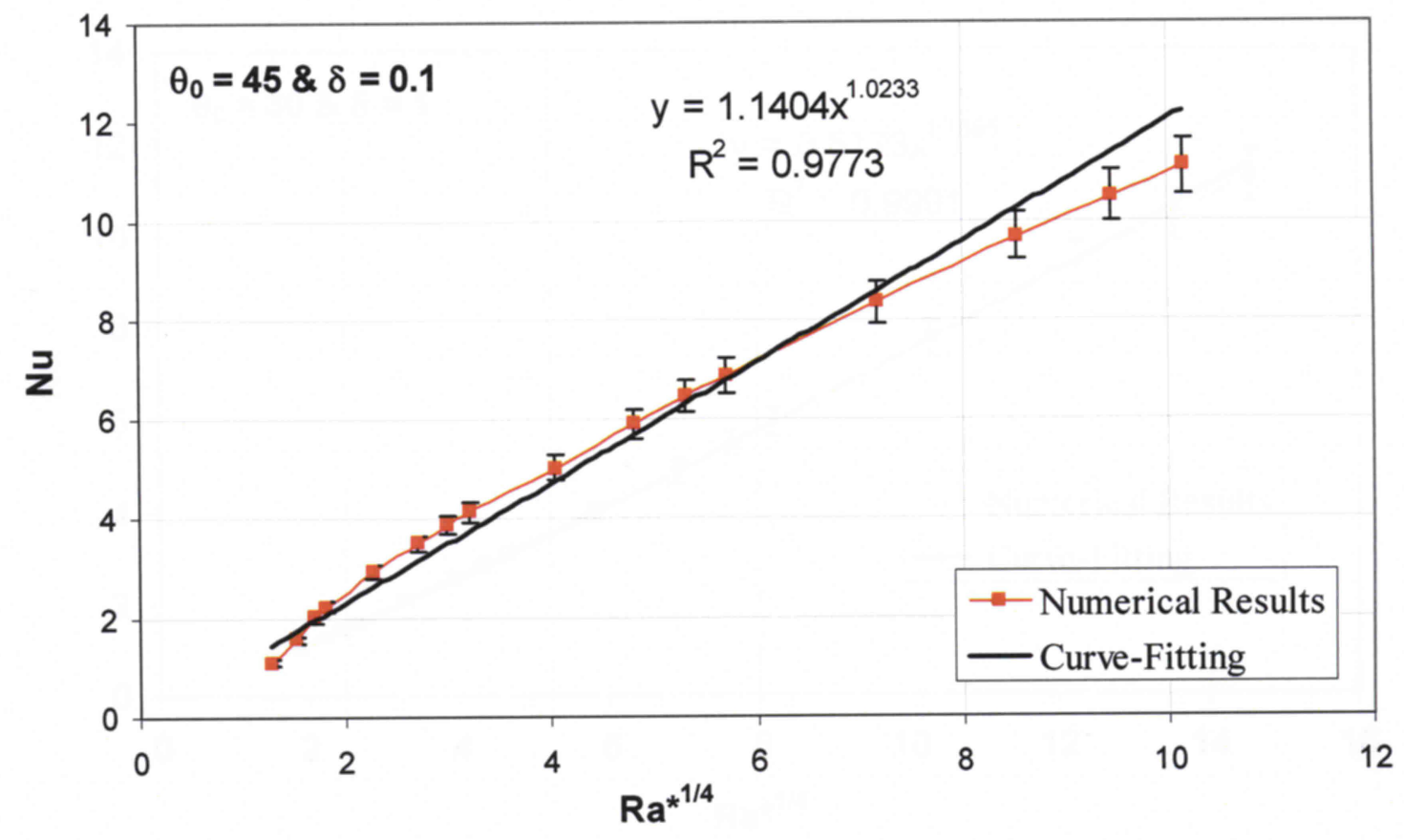

Figure A.15: Curve-fitting to the numerical results for $\theta_{0}=45^{\circ}$ and $\delta=0.1$ 


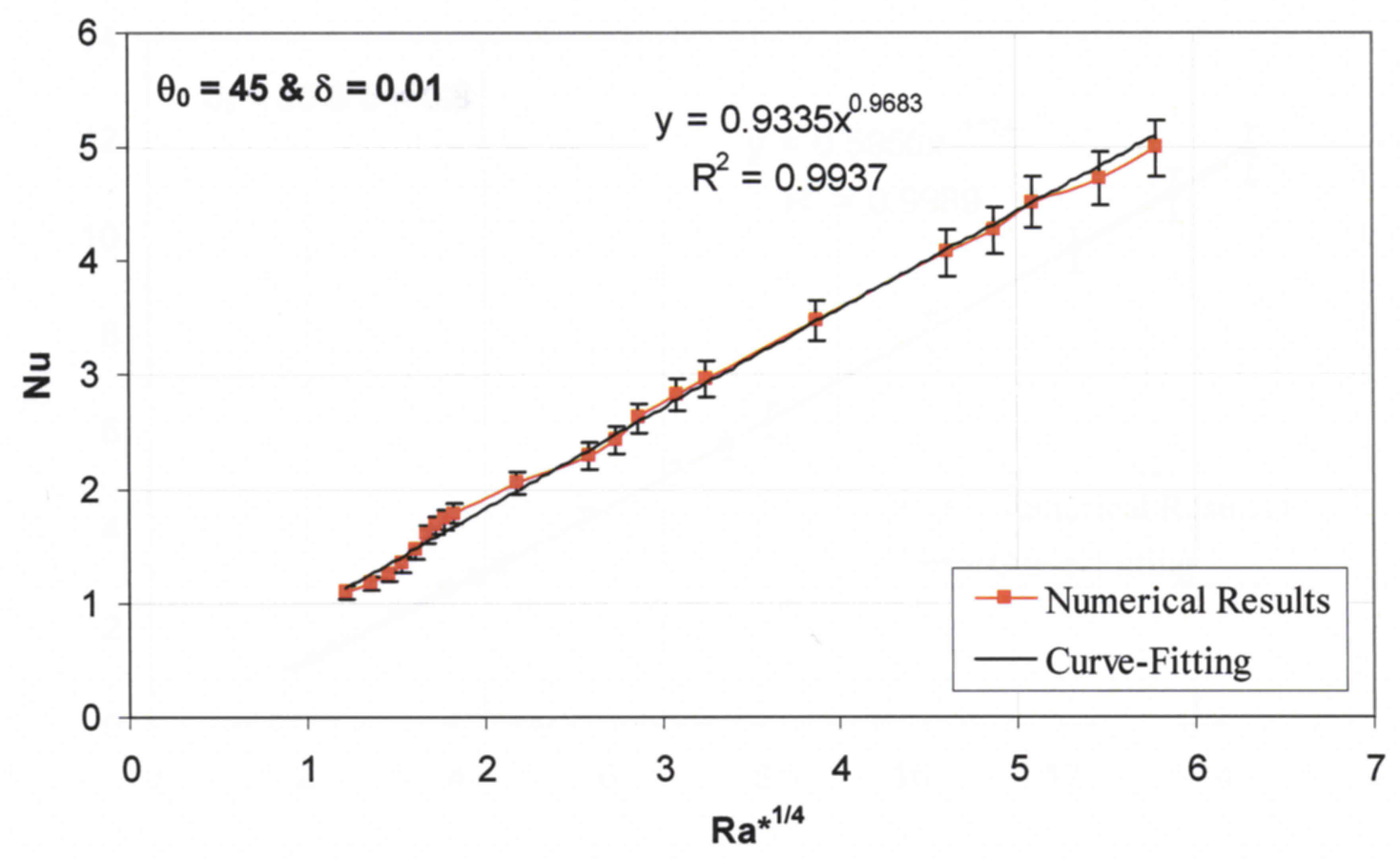

Figure A.16: Curve-fitting to the numerical results for $\theta_{0}=45^{\circ}$ and $\delta=0.01$

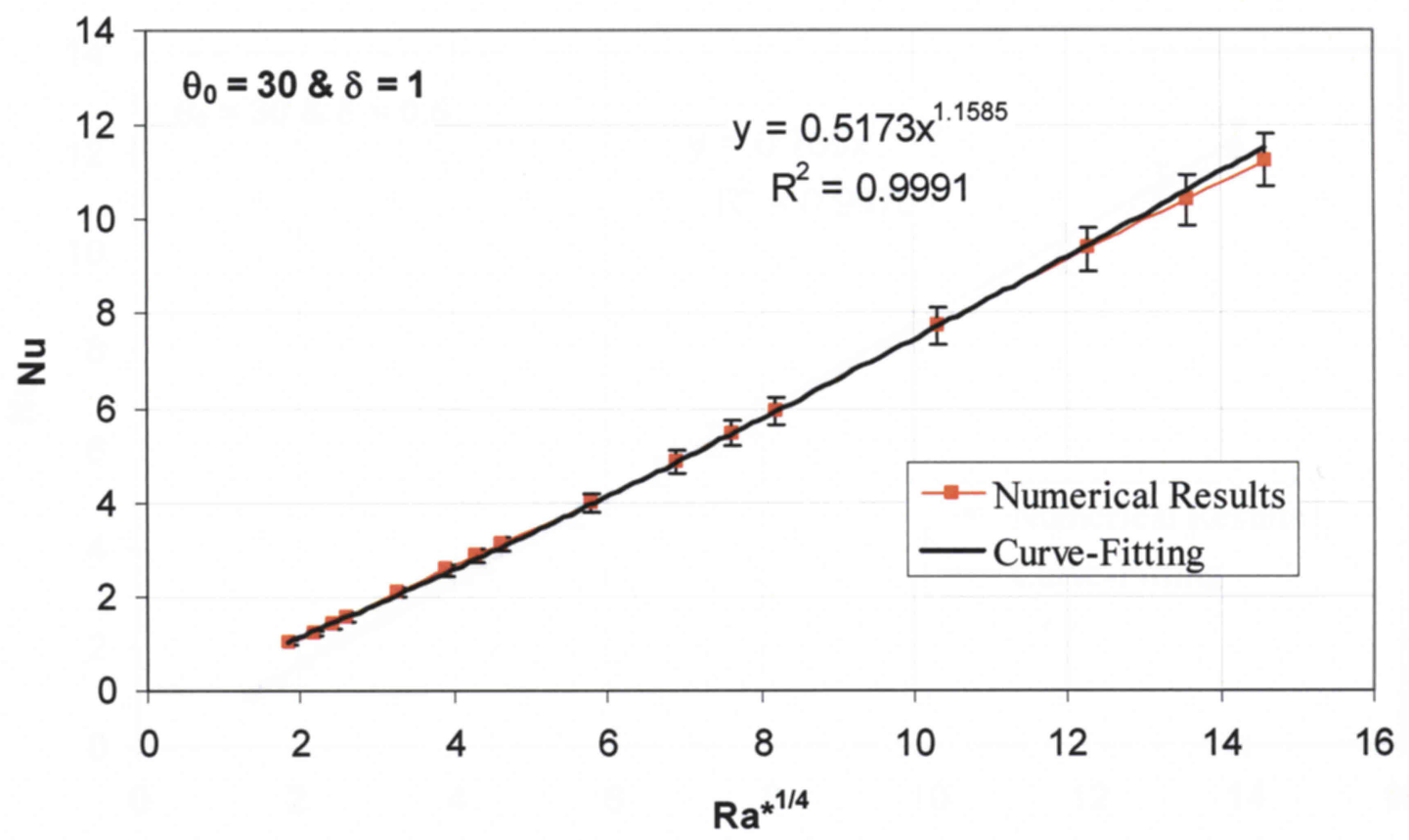

Figure A.17: Curve-fitting to the numerical results for $\theta_{0}=30^{\circ}$ and $\delta=1$ 


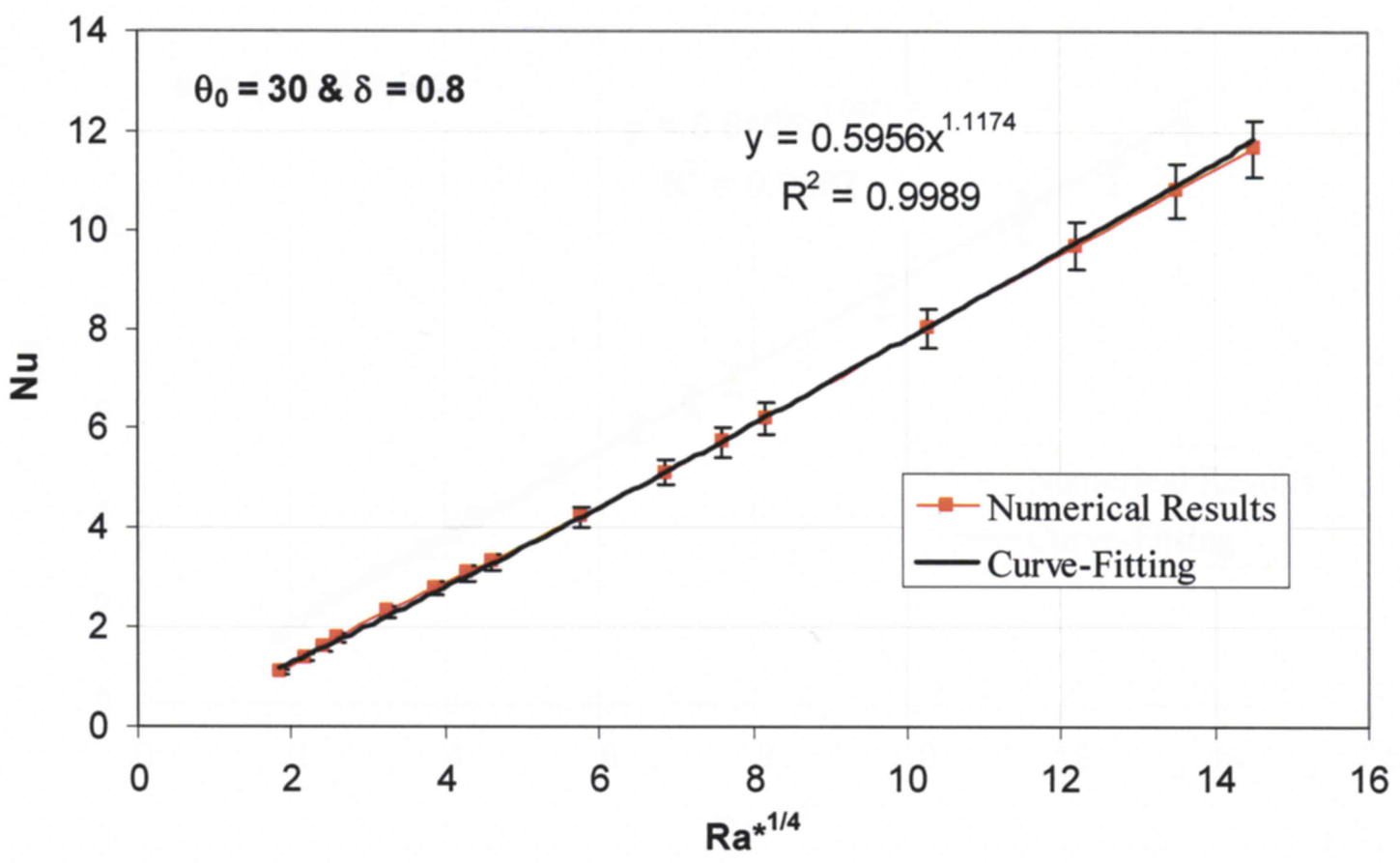

Figure A.18: Curve-fitting to the numerical results for $\theta_{0}=30^{\circ}$ and $\delta=0.8$



Figure A.19: Curve-fitting to the numerical results for $\theta_{0}=30^{\circ}$ and $\delta=0.5$ 




Figure A.20: Curve-fitting to the numerical results for $\theta_{0}=30^{\circ}$ and $\delta=0.4$

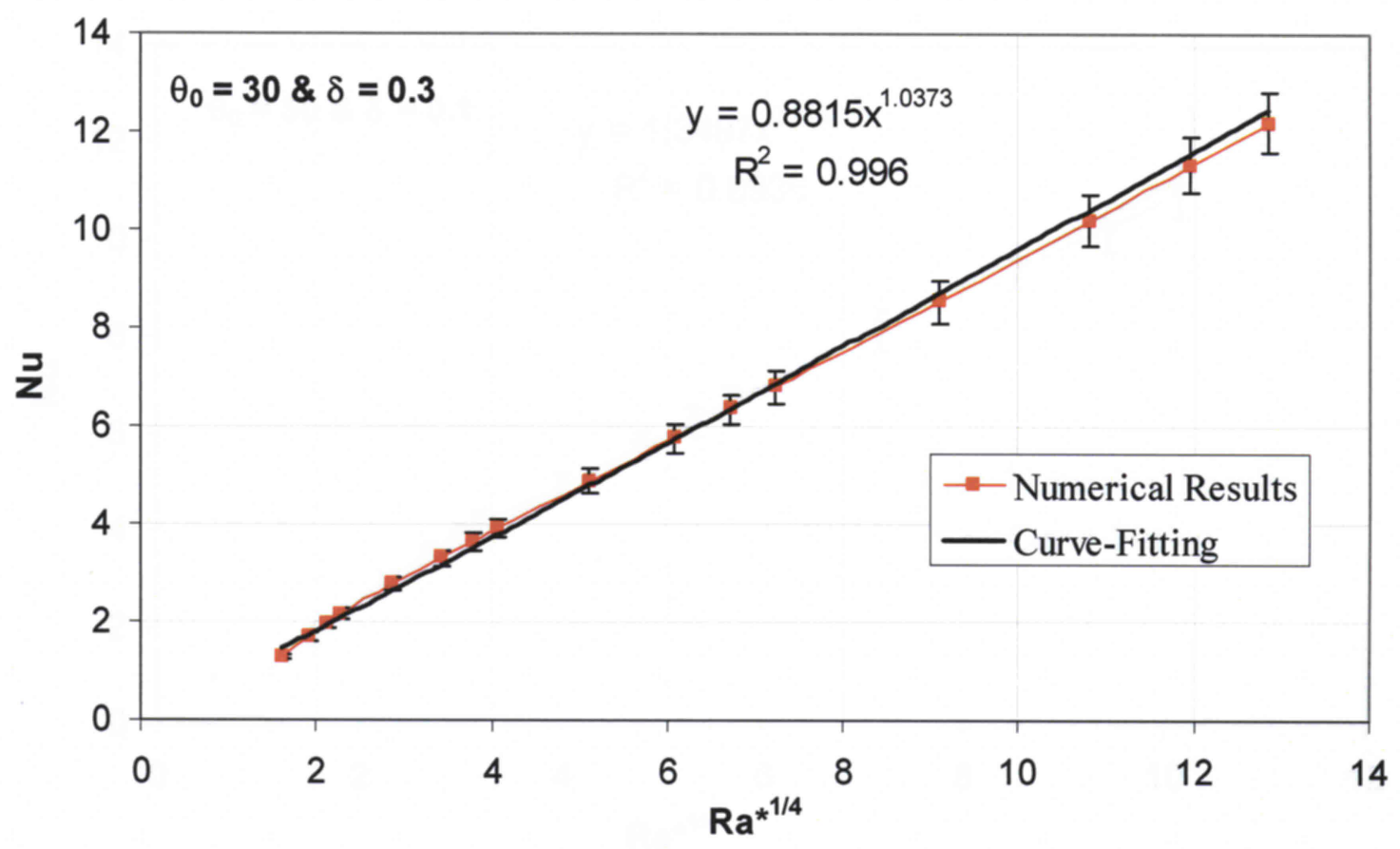

Figure A.21: Curve-fitting to the numerical results for $\theta_{0}=30^{\circ}$ and $\delta=0.3$ 


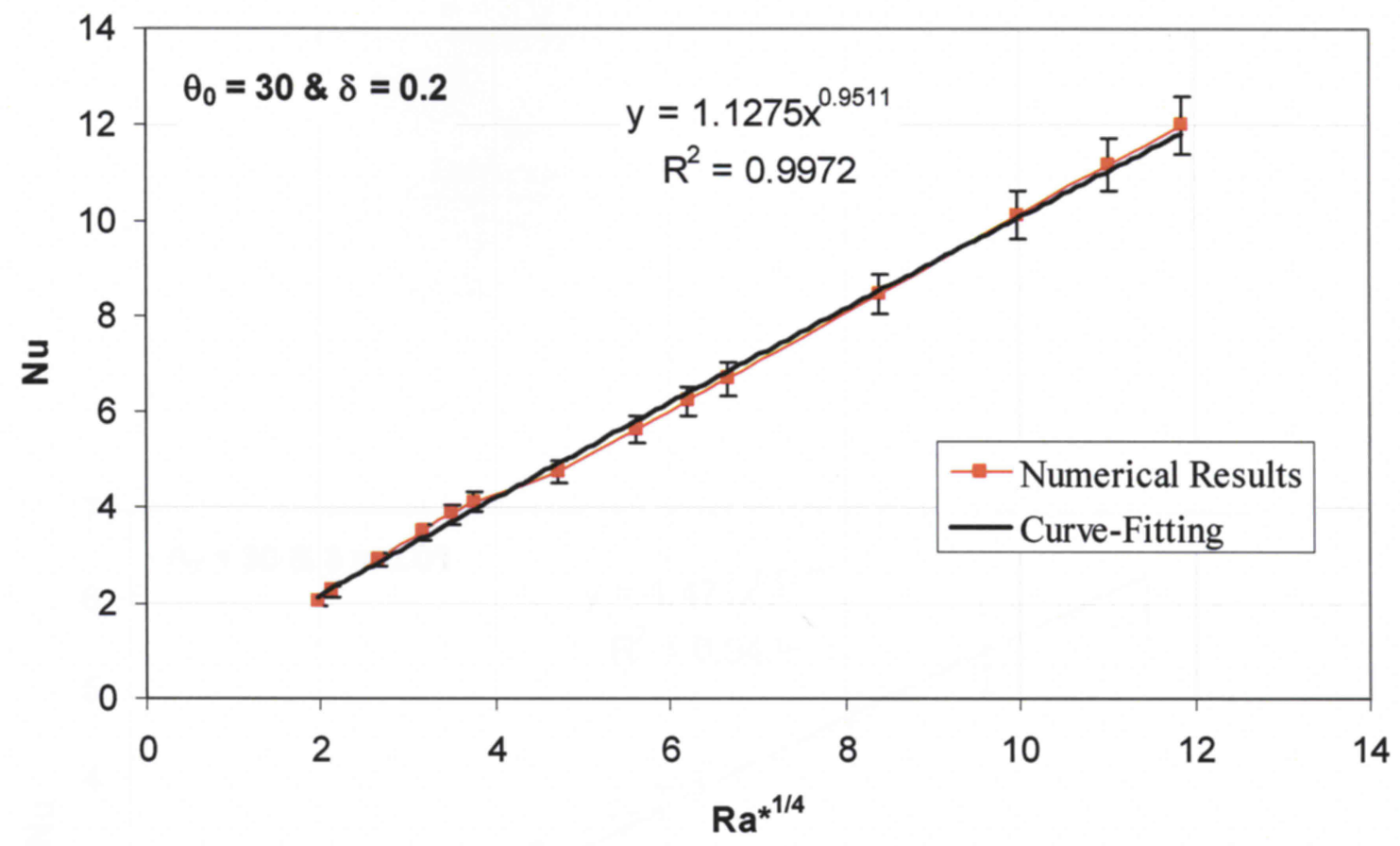

Figure A.22: Curve-fitting to the numerical results for $\theta_{0}=30^{\circ}$ and $\delta=0.2$

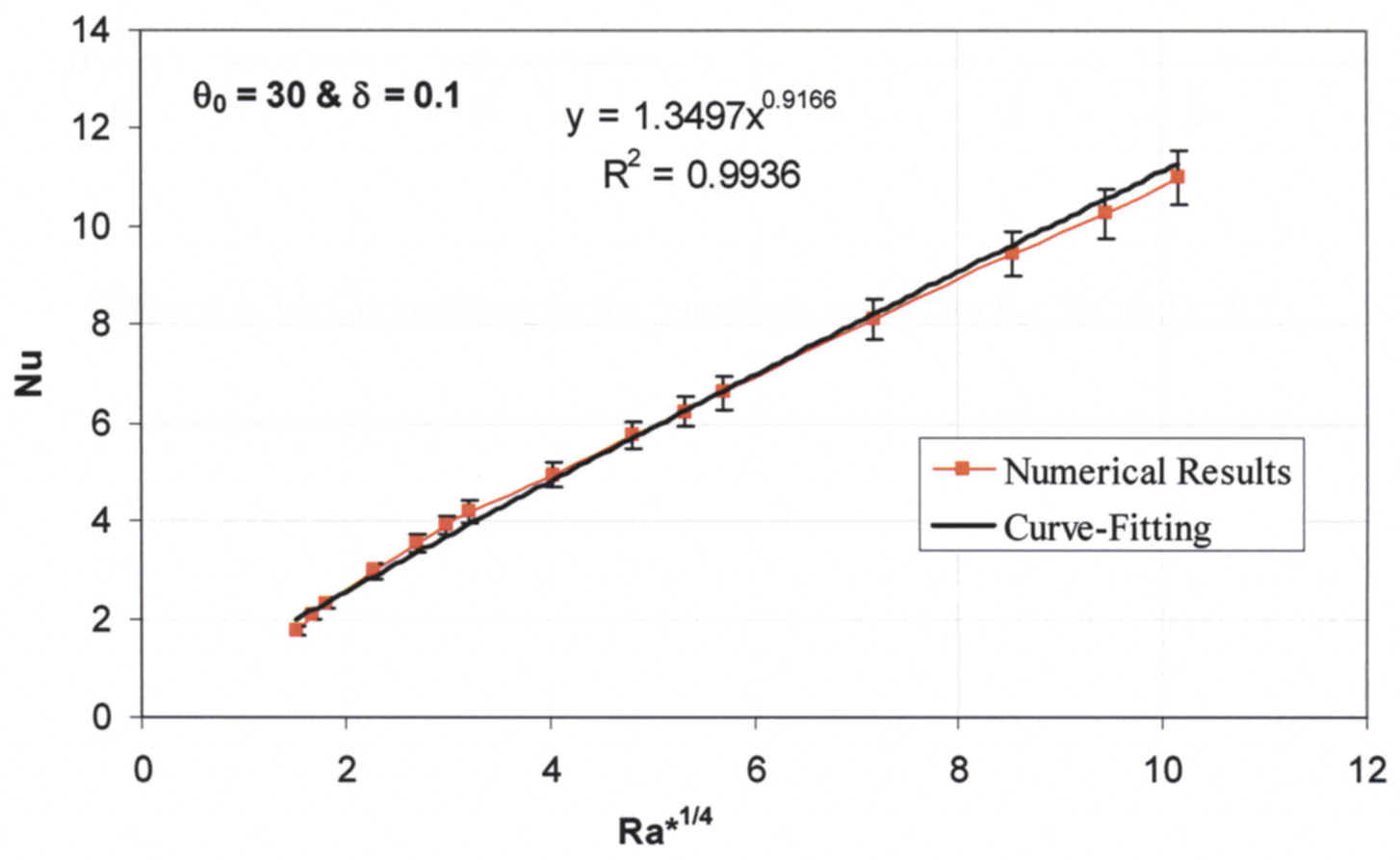

Figure A.23: Curve-fitting to the numerical results for $\theta_{0}=30^{\circ}$ and $\delta=0.1$ 


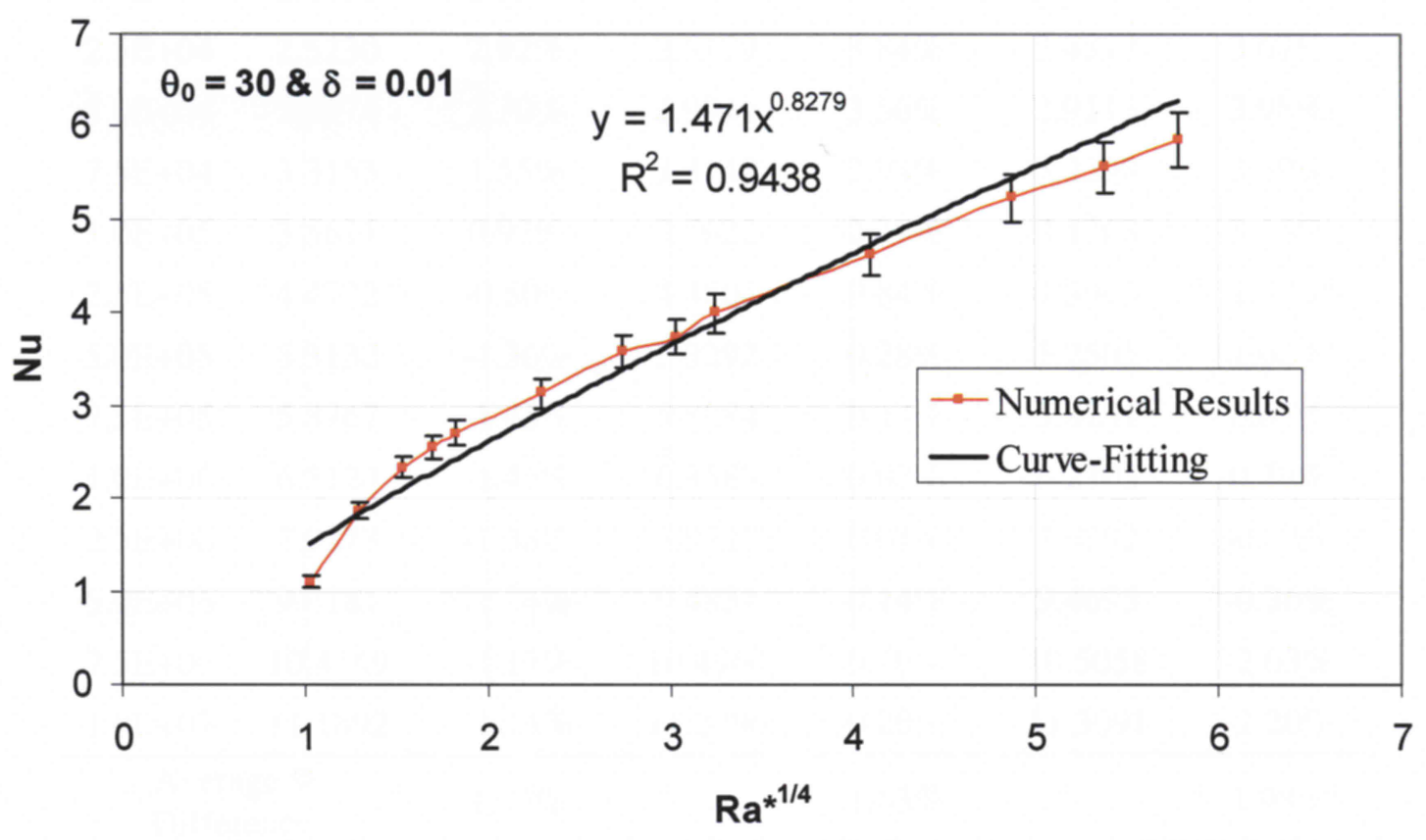

Figure A.24: Curve-fitting to the numerical results for $\theta_{0}=30^{\circ}$ and $\delta=0.01$ 
Table A.7: Curve-fitting to Nusselt number and percentage difference for $\theta_{0}=90^{\circ}$

\begin{tabular}{|c|c|c|c|c|c|c|}
\hline \multirow[b]{2}{*}{ Gr } & \multicolumn{2}{|c|}{$\delta=1$} & \multicolumn{2}{|c|}{$\delta=0.8$} & \multicolumn{2}{|c|}{$\delta=\mathbf{0 . 5}$} \\
\hline & $\mathrm{Nu}$ & $\begin{array}{c}\% \\
\text { Difference }\end{array}$ & $\mathrm{Nu}$ & $\begin{array}{c}\% \\
\text { Difference }\end{array}$ & $\mathrm{Nu}$ & $\begin{array}{c}\% \\
\text { Difference }\end{array}$ \\
\hline $1.0 \mathrm{E}+03$ & 1.1334 & $-3.91 \%$ & 1.1250 & $-3.78 \%$ & 1.0689 & $-0.52 \%$ \\
\hline $2.5 \mathrm{E}+03$ & 1.4233 & $-4.06 \%$ & 1.4150 & $-4.97 \%$ & 1.3516 & $-6.28 \%$ \\
\hline $5.0 \mathrm{E}+03$ & 1.6910 & $0.03 \%$ & 1.6830 & $-0.39 \%$ & 1.6142 & $-2.50 \%$ \\
\hline $7.5 \mathrm{E}+03$ & 1.8703 & $1.55 \%$ & 1.8628 & $1.52 \%$ & 1.7908 & $-0.15 \%$ \\
\hline $1.0 \mathrm{E}+04$ & 2.0090 & $2.23 \%$ & 2.0019 & $2.46 \%$ & 1.9278 & $1.17 \%$ \\
\hline $2.5 \mathrm{E}+04$ & 2.5230 & $2.92 \%$ & 2.5179 & $3.84 \%$ & 2.4377 & $3.67 \%$ \\
\hline $5.0 \mathrm{E}+04$ & 2.9974 & $2.30 \%$ & 2.9949 & $3.56 \%$ & 2.9113 & $3.98 \%$ \\
\hline $7.5 \mathrm{E}+04$ & 3.3153 & $1.55 \%$ & 3.3148 & $2.94 \%$ & 3.2298 & $3.59 \%$ \\
\hline $1.0 \mathrm{E}+05$ & 3.5611 & $0.92 \%$ & 3.5622 & $2.39 \%$ & 3.4768 & $3.15 \%$ \\
\hline $2.5 \mathrm{E}+05$ & 4.4722 & $-0.80 \%$ & 4.4805 & $0.84 \%$ & 4.3965 & $1.77 \%$ \\
\hline $5.0 \mathrm{E}+05$ & 5.3132 & $-1.36 \%$ & 5.3292 & $0.28 \%$ & 5.2505 & $1.02 \%$ \\
\hline $7.5 \mathrm{E}+05$ & 5.8767 & $-1.45 \%$ & 5.8984 & $0.13 \%$ & 5.8251 & $0.65 \%$ \\
\hline $1.0 \mathrm{E}+06$ & 6.3124 & $-1.46 \%$ & 6.3388 & $0.07 \%$ & 6.2705 & $0.39 \%$ \\
\hline $2.5 \mathrm{E}+06$ & 7.9273 & $-1.38 \%$ & 7.9727 & $0.01 \%$ & 7.9292 & $-0.16 \%$ \\
\hline $5.0 \mathrm{E}+06$ & 9.4181 & $-1.24 \%$ & 9.4831 & $0.14 \%$ & 9.4695 & $-0.36 \%$ \\
\hline $7.5 \mathrm{E}+06$ & 10.4169 & $-1.17 \%$ & 10.4960 & $0.19 \%$ & 10.5058 & $-2.03 \%$ \\
\hline $1.0 \mathrm{E}+07$ & 11.1892 & $-1.14 \%$ & 11.2796 & $0.20 \%$ & 11.3091 & $-2.20 \%$ \\
\hline $\begin{array}{l}\text { Aver } \\
\text { Diffe }\end{array}$ & $\%$ & $1.73 \%$ & & $1.63 \%$ & & $1.98 \%$ \\
\hline
\end{tabular}


Table A.7: Continued

\begin{tabular}{c|cc|cc|cc}
\hline \multirow{2}{*}{$\mathbf{G r}$} & \multicolumn{2}{|c|}{$\delta=\mathbf{0 . 4}$} & \multicolumn{2}{c|}{$\delta=\mathbf{0 . 3}$} & \multicolumn{2}{c}{$\delta=\mathbf{0 . 2}$} \\
& $\mathbf{N u}$ & $\begin{array}{c}\text { \% } \\
\text { Difference }\end{array}$ & $\mathbf{N u}$ & $\begin{array}{c}\text { \% } \\
\text { Difference }\end{array}$ & Nu & $\begin{array}{c}\text { \% } \\
\text { Difference }\end{array}$ \\
\hline $1.0 \mathrm{E}+03$ & 1.0284 & $2.36 \%$ & 1.0000 & $3.99 \%$ & 1.0000 & $2.74 \%$ \\
$2.5 \mathrm{E}+03$ & 1.3044 & $-5.98 \%$ & 1.2376 & $-4.77 \%$ & 1.2321 & $-9.74 \%$ \\
$5.0 \mathrm{E}+03$ & 1.5613 & $-3.49 \%$ & 1.4836 & $-4.44 \%$ & 1.4744 & $-2.93 \%$ \\
\hline $7.5 \mathrm{E}+03$ & 1.7345 & $-1.23 \%$ & 1.6496 & $-2.51 \%$ & 1.6376 & $3.08 \%$ \\
\hline $1.0 \mathrm{E}+04$ & 1.8688 & $0.15 \%$ & 1.7786 & $-1.15 \%$ & 1.7643 & $5.98 \%$ \\
$2.5 \mathrm{E}+04$ & 2.3703 & $2.99 \%$ & 2.2603 & $2.03 \%$ & 2.2368 & $9.52 \%$ \\
\hline $5.0 \mathrm{E}+04$ & 2.8371 & $3.55 \%$ & 2.7096 & $2.97 \%$ & 2.6766 & $-4.90 \%$ \\
\hline $7.5 \mathrm{E}+04$ & 3.1518 & $3.21 \%$ & 3.0128 & $2.88 \%$ & 2.9729 & $-4.45 \%$ \\
$1.0 \mathrm{E}+05$ & 3.3960 & $2.79 \%$ & 3.2483 & $2.52 \%$ & 3.2029 & $-4.50 \%$ \\
$2.5 \mathrm{E}+05$ & 4.3071 & $1.36 \%$ & 4.1281 & $1.01 \%$ & 4.0606 & $-5.74 \%$ \\
\hline $5.0 \mathrm{E}+05$ & 5.1555 & $0.46 \%$ & 4.9487 & $0.09 \%$ & 4.8590 & $-6.62 \%$ \\
\hline $7.5 \mathrm{E}+05$ & 5.7273 & $-0.01 \%$ & 5.5025 & $-0.35 \%$ & 5.3970 & $6.21 \%$ \\
$1.0 \mathrm{E}+06$ & 6.1710 & $-0.33 \%$ & 5.9325 & $-0.89 \%$ & 5.8144 & $5.57 \%$ \\
$2.5 \mathrm{E}+06$ & 7.8267 & $-1.23 \%$ & 7.5394 & $0.15 \%$ & 7.3715 & $-3.08 \%$ \\
\hline $5.0 \mathrm{E}+06$ & 9.3684 & $-2.99 \%$ & 9.0382 & $-1.14 \%$ & 8.8209 & $1.32 \%$ \\
\hline $7.5 \mathrm{E}+06$ & 10.4074 & $-3.37 \%$ & 10.0495 & $-1.78 \%$ & 9.7975 & $0.71 \%$ \\
$1.0 \mathrm{E}+07$ & 11.2137 & $-3.65 \%$ & 10.8349 & $-2.34 \%$ & 10.5553 & $-0.38 \%$ \\
\hline Average \% & $2.30 \%$ & & $2.06 \%$ & & $4.56 \%$ \\
\hline Difference: & & & & & &
\end{tabular}


Table A.7: Continued

\begin{tabular}{c|cc|cc}
\hline \multirow{2}{*}{ Gr } & \multicolumn{2}{|c}{$\delta=\mathbf{0 . 1}$} & \multicolumn{2}{c}{$\delta=\mathbf{0 . 0 1}$} \\
\cline { 2 - 5 } & $\mathbf{N u}$ & $\begin{array}{c}\% \\
\text { Difference }\end{array}$ & Nu & $\begin{array}{c}\% \\
\text { Difference }\end{array}$ \\
\hline $1.0 \mathrm{E}+03$ & 1.0000 & $1.38 \%$ & 1.0000 & $0.08 \%$ \\
$2.5 \mathrm{E}+03$ & 1.1514 & $-8.55 \%$ & 1.0000 & $0.42 \%$ \\
$5.0 \mathrm{E}+03$ & 1.3714 & $-11.26 \%$ & 1.0000 & $1.17 \%$ \\
\hline $7.5 \mathrm{E}+03$ & 1.5192 & $-3.55 \%$ & 1.0000 & $1.96 \%$ \\
\hline $1.0 \mathrm{E}+04$ & 1.6336 & $2.91 \%$ & 1.0000 & $2.74 \%$ \\
$2.5 \mathrm{E}+04$ & 2.0585 & $10.58 \%$ & 1.0866 & $0.37 \%$ \\
\hline $5.0 \mathrm{E}+04$ & 2.4520 & $8.45 \%$ & 1.2893 & $0.75 \%$ \\
\hline $7.5 \mathrm{E}+04$ & 2.7161 & $1.63 \%$ & 1.4249 & $2.09 \%$ \\
$1.0 \mathrm{E}+05$ & 2.9207 & $0.89 \%$ & 1.5297 & $1.13 \%$ \\
$2.5 \mathrm{E}+05$ & 3.6804 & $2.74 \%$ & 1.9178 & $-2.90 \%$ \\
\hline $5.0 \mathrm{E}+05$ & 4.3839 & $2.16 \%$ & 2.2755 & $-2.09 \%$ \\
\hline $7.5 \mathrm{E}+05$ & 4.8562 & $2.02 \%$ & 2.5149 & $-1.72 \%$ \\
$1.0 \mathrm{E}+06$ & 5.2218 & $3.68 \%$ & 2.6999 & $-1.22 \%$ \\
$2.5 \mathrm{E}+06$ & 6.5802 & $-1.72 \%$ & 3.3847 & $0.51 \%$ \\
\hline $5.0 \mathrm{E}+06$ & 7.8379 & $-3.64 \%$ & 4.0160 & $1.68 \%$ \\
\hline $7.5 \mathrm{E}+06$ & 8.6823 & $-4.61 \%$ & 4.4386 & $0.70 \%$ \\
$1.0 \mathrm{E}+07$ & 9.3360 & $-4.80 \%$ & 4.7651 & $0.52 \%$ \\
\hline Average \% & $4.39 \%$ & & $1.30 \%$ \\
\hline Difference: & & & \\
\hline
\end{tabular}


Table A.8: Curve-fitting to Nusselt number and percentage difference for $\theta_{0}=45^{\circ}$

\begin{tabular}{|c|c|c|c|c|c|c|}
\hline \multirow[b]{2}{*}{ Gr } & \multicolumn{2}{|c|}{$\delta=1$} & \multicolumn{2}{|c|}{$\delta=0.8$} & \multicolumn{2}{|c|}{$\delta=0.5$} \\
\hline & $\mathrm{Nu}$ & $\begin{array}{c}\% \\
\text { Difference } \\
\end{array}$ & $\mathrm{Nu}$ & $\begin{array}{c}\% \\
\text { Difference } \\
\end{array}$ & $\mathrm{Nu}$ & $\begin{array}{c}\% \\
\text { Difference } \\
\end{array}$ \\
\hline $1.0 \mathrm{E}+03$ & 1.0220 & $0.81 \%$ & 1.1074 & $-6.09 \%$ & 1.1584 & $-10.03 \%$ \\
\hline $2.5 \mathrm{E}+03$ & 1.2939 & $-4.14 \%$ & 1.4020 & $-7.23 \%$ & 1.4666 & $-9.41 \%$ \\
\hline $5.0 \mathrm{E}+03$ & 1.5468 & $1.50 \%$ & 1.6760 & $-0.36 \%$ & 1.7532 & $-0.78 \%$ \\
\hline $7.5 \mathrm{E}+03$ & 1.7170 & $3.72 \%$ & 1.8604 & $1.98 \%$ & 1.9461 & $2.13 \%$ \\
\hline $1.0 \mathrm{E}+04$ & 1.8490 & $4.59 \%$ & 2.0035 & $2.87 \%$ & 2.0958 & $3.35 \%$ \\
\hline $2.5 \mathrm{E}+04$ & 2.3410 & $5.00 \%$ & 2.5366 & $3.23 \%$ & 2.6535 & $4.50 \%$ \\
\hline $5.0 \mathrm{E}+04$ & 2.7985 & $4.57 \%$ & 3.0323 & $2.37 \%$ & 3.1720 & $3.92 \%$ \\
\hline $7.5 \mathrm{E}+04$ & 3.1065 & $3.83 \%$ & 3.3660 & $1.70 \%$ & 3.5211 & $3.28 \%$ \\
\hline $1.0 \mathrm{E}+05$ & 3.3453 & $3.50 \%$ & 3.6248 & $1.22 \%$ & 3.7918 & $2.73 \%$ \\
\hline $2.5 \mathrm{E}+05$ & 4.2355 & $2.72 \%$ & 4.5894 & $-0.15 \%$ & 4.8008 & $0.81 \%$ \\
\hline $5.0 \mathrm{E}+05$ & 5.0632 & $2.56 \%$ & 5.4862 & $-0.80 \%$ & 5.7389 & $-0.39 \%$ \\
\hline $7.5 \mathrm{E}+05$ & 5.6204 & $2.62 \%$ & 6.0899 & $-1.02 \%$ & 6.3705 & $-0.95 \%$ \\
\hline $1.0 \mathrm{E}+06$ & 6.0526 & $2.71 \%$ & 6.5582 & $-1.10 \%$ & 6.8603 & $-1.25 \%$ \\
\hline $2.5 \mathrm{E}+06$ & 7.6632 & $3.48 \%$ & 8.3034 & $-0.56 \%$ & 8.6859 & $-1.48 \%$ \\
\hline $5.0 \mathrm{E}+06$ & 9.1606 & $4.04 \%$ & 9.9259 & $-0.31 \%$ & 10.3832 & $-1.47 \%$ \\
\hline $7.5 \mathrm{E}+06$ & 10.1688 & $4.20 \%$ & 11.0183 & $-0.30 \%$ & 11.5259 & $-1.52 \%$ \\
\hline $1.0 \mathrm{E}+07$ & 10.9506 & $4.25 \%$ & 11.8655 & $-0.35 \%$ & 12.4121 & $-1.61 \%$ \\
\hline $\begin{array}{l}\text { Aver } \\
\text { Diffe }\end{array}$ & $\begin{array}{c}\% \\
\text { ce: }\end{array}$ & $3.43 \%$ & & $1.86 \%$ & & $2.92 \%$ \\
\hline
\end{tabular}


Table A.8: Continued

\begin{tabular}{|c|c|c|c|c|c|c|}
\hline \multirow[b]{2}{*}{ Gr } & \multicolumn{2}{|c|}{$\delta=0.4$} & \multicolumn{2}{|c|}{$\delta=0.3$} & \multicolumn{2}{|c|}{$\delta=0.2$} \\
\hline & $\mathrm{Nu}$ & $\begin{array}{c}\% \\
\text { Difference } \\
\end{array}$ & $\mathrm{Nu}$ & $\begin{array}{c}\% \\
\text { Difference } \\
\end{array}$ & $\mathrm{Nu}$ & $\begin{array}{c}\% \\
\text { Difference } \\
\end{array}$ \\
\hline $1.0 \mathrm{E}+03$ & 1.1294 & $-7.62 \%$ & 1.1835 & $-13.55 \%$ & 2.4962 & $-141.73 \%$ \\
\hline $2.5 \mathrm{E}+03$ & 1.4299 & $-9.10 \%$ & 1.4985 & $-18.90 \%$ & 3.1186 & $-26.13 \%$ \\
\hline $5.0 \mathrm{E}+03$ & 1.7094 & $-0.42 \%$ & 1.7913 & $-2.01 \%$ & 3.6906 & $-2.89 \%$ \\
\hline $7.5 \mathrm{E}+03$ & 1.8975 & $2.93 \%$ & 1.9884 & $2.85 \%$ & 4.0727 & $4.35 \%$ \\
\hline $1.0 \mathrm{E}+04$ & 2.0434 & $4.42 \%$ & 2.1413 & $4.74 \%$ & 4.3675 & $6.95 \%$ \\
\hline $2.5 \mathrm{E}+04$ & 2.5871 & $6.20 \%$ & 2.7111 & $6.92 \%$ & 5.4565 & $10.33 \%$ \\
\hline $5.0 \mathrm{E}+04$ & 3.0927 & $5.90 \%$ & 3.2409 & $6.84 \%$ & 6.4573 & $10.17 \%$ \\
\hline $7.5 \mathrm{E}+04$ & 3.4330 & $5.35 \%$ & 3.5975 & $6.34 \%$ & 7.1258 & $6.05 \%$ \\
\hline $1.0 \mathrm{E}+05$ & 3.6970 & $4.85 \%$ & 3.8741 & $5.79 \%$ & 7.6416 & $5.70 \%$ \\
\hline $2.5 \mathrm{E}+05$ & 4.6808 & $3.11 \%$ & 4.9051 & $2.77 \%$ & 9.5470 & $4.58 \%$ \\
\hline $5.0 \mathrm{E}+05$ & 5.5955 & $1.96 \%$ & 5.8636 & $-4.56 \%$ & 11.2980 & $4.05 \%$ \\
\hline $7.5 \mathrm{E}+05$ & 6.2112 & $1.40 \%$ & 6.5089 & $-5.00 \%$ & 12.4676 & $3.49 \%$ \\
\hline $1.0 \mathrm{E}+06$ & 6.6888 & $1.27 \%$ & 7.0093 & $-5.16 \%$ & 13.3702 & $3.47 \%$ \\
\hline $2.5 \mathrm{E}+06$ & 8.4688 & $1.09 \%$ & 8.8745 & $-5.57 \%$ & 16.7038 & $0.55 \%$ \\
\hline $5.0 \mathrm{E}+06$ & 10.1236 & $0.80 \%$ & 10.6087 & $-6.31 \%$ & 19.7675 & $-2.03 \%$ \\
\hline $7.5 E+06$ & 11.2377 & $0.51 \%$ & 11.7762 & $-6.21 \%$ & 21.8139 & $-2.29 \%$ \\
\hline $1.0 \mathrm{E}+07$ & 12.1018 & $0.23 \%$ & 12.6817 & $-6.37 \%$ & 23.3931 & $-2.34 \%$ \\
\hline $\begin{array}{l}\text { Aver: } \\
\text { Diffe }\end{array}$ & $\begin{array}{l}\text { e } \% \\
\text { nce: }\end{array}$ & $3.36 \%$ & & $6.46 \%$ & & $5.96 \%$ \\
\hline
\end{tabular}


Table A.8: Continued

\begin{tabular}{|c|c|c|c|c|c|}
\hline \multirow{2}{*}{$\mathbf{G r}$} & \multicolumn{2}{|c|}{$\delta=0.1$} & \multirow{2}{*}{ Gr } & \multicolumn{2}{|c|}{$\delta=0.01$} \\
\hline & $\mathrm{Nu}$ & $\begin{array}{c}\% \\
\text { Difference } \\
\end{array}$ & & $\mathrm{Nu}$ & $\begin{array}{c}\% \\
\text { Difference }\end{array}$ \\
\hline $1.0 \mathrm{E}+03$ & 1.2368 & $-21.46 \%$ & $1.0 \mathrm{E}+03$ & 1.0000 & $0.09 \%$ \\
\hline $2.5 \mathrm{E}+03$ & 1.5452 & $-40.62 \%$ & $2.5 \mathrm{E}+03$ & 1.0000 & $0.51 \%$ \\
\hline $5.0 \mathrm{E}+03$ & 1.8287 & $-16.78 \%$ & $5.0 \mathrm{E}+03$ & 1.0000 & $1.69 \%$ \\
\hline $7.5 \mathrm{E}+03$ & 2.0180 & $-0.37 \%$ & $7.5 \mathrm{E}+03$ & 1.0000 & $3.04 \%$ \\
\hline $1.0 \mathrm{E}+04$ & 2.1640 & $3.17 \%$ & $8.5 \mathrm{E}+03$ & 1.0000 & $3.57 \%$ \\
\hline $2.5 \mathrm{E}+04$ & 2.7036 & $7.62 \%$ & $1.0 \mathrm{E}+04$ & 1.0000 & $4.36 \%$ \\
\hline $5.0 \mathrm{E}+04$ & 3.1995 & $8.65 \%$ & $2.0 \mathrm{E}+04$ & 1.0587 & $3.84 \%$ \\
\hline $7.5 \mathrm{E}+04$ & 3.5307 & $8.88 \%$ & $3.0 \mathrm{E}+04$ & 1.1683 & $0.04 \%$ \\
\hline $1.0 \mathrm{E}+05$ & 3.7863 & $8.77 \%$ & $4.0 \mathrm{E}+04$ & 1.2529 & $0.41 \%$ \\
\hline $2.5 \mathrm{E}+05$ & 4.7304 & $6.05 \%$ & $5.0 \mathrm{E}+04$ & 1.3227 & $2.00 \%$ \\
\hline $5.0 \mathrm{E}+05$ & 5.5980 & $5.25 \%$ & $6.0 \mathrm{E}+04$ & 1.3826 & $5.62 \%$ \\
\hline $7.5 \mathrm{E}+05$ & 6.1775 & $4.38 \%$ & $7.0 \mathrm{E}+04$ & 1.4353 & $10.40 \%$ \\
\hline $1.0 \mathrm{E}+06$ & 6.6247 & $3.48 \%$ & $8.0 \mathrm{E}+04$ & 1.4827 & $11.89 \%$ \\
\hline $2.5 \mathrm{E}+06$ & 8.2765 & $0.78 \%$ & $9.0 \mathrm{E}+04$ & 1.5257 & $12.46 \%$ \\
\hline $5.0 \mathrm{E}+06$ & 9.7945 & $-1.16 \%$ & $1.0 \mathrm{E}+05$ & 1.5653 & $12.75 \%$ \\
\hline $7.5 \mathrm{E}+06$ & 10.8084 & $-3.06 \%$ & $2.0 \mathrm{E}+05$ & 1.8524 & $10.09 \%$ \\
\hline $1.0 \mathrm{E}+07$ & 11.5909 & $-4.42 \%$ & $4.0 \mathrm{E}+05$ & 2.1921 & $4.47 \%$ \\
\hline \multicolumn{2}{|c|}{$\begin{array}{l}\text { Average \% } \\
\text { Difference: }\end{array}$} & $8.52 \%$ & $5.0 \mathrm{E}+05$ & 2.3142 & $4.81 \%$ \\
\hline & & & $6.0 \mathrm{E}+05$ & 2.4190 & $7.68 \%$ \\
\hline & & & $8.0 \mathrm{E}+05$ & 2.5941 & $8.12 \%$ \\
\hline & & & $1.0 \mathrm{E}+06$ & 2.7387 & $7.49 \%$ \\
\hline & & & $2.0 \mathrm{E}+06$ & 3.2410 & $6.69 \%$ \\
\hline & & & $4.0 \mathrm{E}+06$ & 3.8354 & $5.79 \%$ \\
\hline & & & $5.0 \mathrm{E}+06$ & 4.0491 & $5.09 \%$ \\
\hline & & & $6.0 \mathrm{E}+06$ & 4.2324 & $6.22 \%$ \\
\hline & & & $8.0 \mathrm{E}+06$ & 4.5388 & $4.04 \%$ \\
\hline & & & $1.0 \mathrm{E}+07$ & 4.7917 & $4.06 \%$ \\
\hline & & & $\begin{array}{l}\text { Avera } \\
\text { Differ }\end{array}$ & $\begin{array}{l}\text { ge \% } \\
\text { ence: }\end{array}$ & $5.45 \%$ \\
\hline
\end{tabular}


Table A.9: Curve-fitting to Nusselt number and percentage difference for $\theta_{0}=30^{\circ}$

\begin{tabular}{|c|c|c|c|c|c|c|}
\hline \multirow{2}{*}{ Gr } & \multicolumn{2}{|c|}{$\delta=1$} & \multicolumn{2}{|c|}{$\delta=0.8$} & \multicolumn{2}{|c|}{$\delta=0.5$} \\
\hline & $\mathrm{Nu}$ & $\begin{array}{c}\% \\
\text { Difference }\end{array}$ & $\mathrm{Nu}$ & $\begin{array}{c}\% \\
\text { Difference } \\
\end{array}$ & $\mathrm{Nu}$ & $\begin{array}{c}\% \\
\text { Difference }\end{array}$ \\
\hline $1.0 \mathrm{E}+03$ & 1.0000 & $0.24 \%$ & 1.0000 & $0.65 \%$ & 1.0899 & $-6.48 \%$ \\
\hline $2.5 \mathrm{E}+03$ & 1.0434 & $-1.02 \%$ & 1.1675 & $-6.88 \%$ & 1.3880 & $-10.39 \%$ \\
\hline $5.0 \mathrm{E}+03$ & 1.2754 & $-5.44 \%$ & 1.4165 & $-2.85 \%$ & 1.6666 & $-0.65 \%$ \\
\hline $7.5 \mathrm{E}+03$ & 1.4344 & $-2.42 \%$ & 1.5861 & $0.79 \%$ & 1.8548 & $2.66 \%$ \\
\hline $1.0 \mathrm{E}+04$ & 1.5591 & $-0.24 \%$ & 1.7186 & $2.51 \%$ & 2.0011 & $3.86 \%$ \\
\hline $2.5 \mathrm{E}+04$ & 2.0331 & $3.21 \%$ & 2.2191 & $3.84 \%$ & 2.5486 & $4.32 \%$ \\
\hline $5.0 \mathrm{E}+04$ & 2.4853 & $3.24 \%$ & 2.6925 & $2.87 \%$ & 3.0601 & $3.20 \%$ \\
\hline $7.5 \mathrm{E}+04$ & 2.7952 & $2.78 \%$ & 3.0149 & $2.12 \%$ & 3.4057 & $2.35 \%$ \\
\hline $1.0 \mathrm{E}+05$ & 3.0381 & $2.36 \%$ & 3.2668 & $1.60 \%$ & 3.6744 & $1.71 \%$ \\
\hline $2.5 \mathrm{E}+05$ & 3.9619 & $1.05 \%$ & 4.2181 & $0.38 \%$ & 4.6795 & $-0.18 \%$ \\
\hline $5.0 \mathrm{E}+05$ & 4.8431 & $0.51 \%$ & 5.1179 & $0.03 \%$ & 5.6188 & $-1.22 \%$ \\
\hline $7.5 \mathrm{E}+05$ & 5.4468 & $0.37 \%$ & 5.7307 & $-0.01 \%$ & 6.2534 & $-1.66 \%$ \\
\hline $1.0 \mathrm{E}+06$ & 5.9202 & $0.30 \%$ & 6.2095 & $0.00 \%$ & 6.7467 & $-1.92 \%$ \\
\hline $2.5 \mathrm{E}+06$ & 7.7203 & $-0.05 \%$ & 8.0178 & $-0.10 \%$ & 8.5923 & $-2.60 \%$ \\
\hline $5.0 \mathrm{E}+06$ & 9.4375 & $-0.68 \%$ & 9.7281 & $-0.50 \%$ & 10.3170 & $-2.94 \%$ \\
\hline $7.5 \mathrm{E}+06$ & 10.6139 & $-2.04 \%$ & 10.8929 & $-0.88 \%$ & 11.4822 & $-3.02 \%$ \\
\hline $1.0 \mathrm{E}+07$ & 11.5365 & $-2.59 \%$ & 11.8030 & $-1.24 \%$ & 12.3879 & $-3.06 \%$ \\
\hline $\begin{array}{l}\text { Aver } \\
\text { Diffe }\end{array}$ & $\begin{array}{l}\% \\
\text { ce: }\end{array}$ & $1.68 \%$ & & $1.60 \%$ & & $3.07 \%$ \\
\hline
\end{tabular}


Table A-9: Continued

\begin{tabular}{|c|c|c|c|c|c|c|}
\hline \multirow[b]{2}{*}{$\mathbf{G r}$} & \multicolumn{2}{|c|}{$\delta=0.4$} & \multicolumn{2}{|c|}{$\delta=0.3$} & \multicolumn{2}{|c|}{$\delta=0.2$} \\
\hline & $\mathbf{N u}$ & $\begin{array}{c}\% \\
\text { Difference } \\
\end{array}$ & $\mathrm{Nu}$ & $\begin{array}{c}\% \% \\
\text { Difference } \\
\end{array}$ & $\mathrm{Nu}$ & $\begin{array}{c}\% \\
\text { Difference } \\
\end{array}$ \\
\hline $1.0 \mathrm{E}+03$ & 1.1275 & $-9.61 \%$ & 1.1917 & $-15.85 \%$ & 1.2997 & $-26.93 \%$ \\
\hline $2.5 \mathrm{E}+03$ & 1.4296 & $-11.00 \%$ & 1.5022 & $-18.35 \%$ & 1.6238 & $-32.34 \%$ \\
\hline $5.0 \mathrm{E}+03$ & 1.7108 & $-0.12 \%$ & 1.7898 & $-5.60 \%$ & 1.9218 & $-9.68 \%$ \\
\hline $7.5 \mathrm{E}+03$ & 1.9003 & $3.39 \%$ & 1.9829 & $-1.18 \%$ & 2.1208 & $-3.41 \%$ \\
\hline $1.0 \mathrm{E}+04$ & 2.0473 & $4.75 \%$ & 2.1325 & $0.69 \%$ & 2.2744 & $-0.85 \%$ \\
\hline $2.5 \mathrm{E}+04$ & 2.5959 & $5.80 \%$ & 2.6881 & $3.00 \%$ & 2.8417 & $2.84 \%$ \\
\hline $5.0 \mathrm{E}+04$ & 3.1065 & $5.11 \%$ & 3.2028 & $3.06 \%$ & 3.3631 & $3.77 \%$ \\
\hline $7.5 \mathrm{E}+04$ & 3.4506 & $4.46 \%$ & 3.5484 & $2.78 \%$ & 3.7114 & $3.83 \%$ \\
\hline $1.0 \mathrm{E}+05$ & 3.7176 & $3.93 \%$ & 3.8159 & $2.50 \%$ & 3.9802 & $3.63 \%$ \\
\hline $2.5 \mathrm{E}+05$ & 4.7136 & $2.16 \%$ & 4.8103 & $1.39 \%$ & 4.9730 & $-4.61 \%$ \\
\hline $5.0 \mathrm{E}+05$ & 5.6407 & $0.98 \%$ & 5.7312 & $0.42 \%$ & 5.8854 & $-4.55 \%$ \\
\hline $7.5 \mathrm{E}+05$ & 6.2655 & $0.43 \%$ & 6.3496 & $-0.13 \%$ & 6.4949 & $-4.43 \%$ \\
\hline $1.0 \mathrm{E}+06$ & 6.7503 & $0.07 \%$ & 6.8285 & $-0.46 \%$ & 6.9653 & $-4.27 \%$ \\
\hline $2.5 \mathrm{E}+06$ & 8.5589 & $-0.93 \%$ & 8.6078 & $-0.89 \%$ & 8.7027 & $-2.98 \%$ \\
\hline $5.0 \mathrm{E}+06$ & 10.2424 & $-1.64 \%$ & 10.2557 & $-0.58 \%$ & 10.2995 & $-1.90 \%$ \\
\hline $7.5 \mathrm{E}+06$ & 11.3768 & $-1.96 \%$ & 11.3624 & $-0.33 \%$ & 11.3661 & $-1.53 \%$ \\
\hline $1.0 \mathrm{E}+07$ & 12.2571 & $-2.08 \%$ & 12.2192 & $-0.14 \%$ & 12.1893 & $-1.36 \%$ \\
\hline $\begin{array}{l}\text { Aver } \\
\text { Diffe }\end{array}$ & $\begin{array}{l}\% \\
\text { ce: }\end{array}$ & $3.44 \%$ & & $3.37 \%$ & & $6.64 \%$ \\
\hline
\end{tabular}


Table A-9: Continued

\begin{tabular}{c|c|c|c|c}
\hline \multirow{2}{*}{$\mathbf{G r}$} & \multicolumn{2}{|c|}{$\delta=\mathbf{0 . 1}$} & \multicolumn{2}{c}{$\delta=0.01$} \\
\cline { 2 - 5 } & Nu & $\begin{array}{c}\text { \% } \\
\text { Difference }\end{array}$ & Nu & $\begin{array}{c}\% \\
\text { Difference }\end{array}$ \\
\hline $1.0 \mathrm{E}+03$ & 1.3775 & $-35.75 \%$ & 1.0000 & $0.11 \%$ \\
$2.5 \mathrm{E}+03$ & 1.6973 & $-52.10 \%$ & 1.1288 & $-12.10 \%$ \\
$5.0 \mathrm{E}+03$ & 1.9875 & $-13.03 \%$ & 1.3030 & $-27.28 \%$ \\
\hline $7.5 \mathrm{E}+03$ & 2.1799 & $-4.13 \%$ & 1.4170 & $-36.00 \%$ \\
\hline $1.0 \mathrm{E}+04$ & 2.3275 & $-0.95 \%$ & 1.5039 & $-36.84 \%$ \\
\hline $2.5 \mathrm{E}+04$ & 2.8677 & $3.51 \%$ & 1.8180 & $2.43 \%$ \\
\hline $5.0 \mathrm{E}+04$ & 3.3582 & $5.45 \%$ & 2.0984 & $9.97 \%$ \\
\hline $7.5 \mathrm{E}+04$ & 3.6831 & $6.05 \%$ & 2.2821 & $10.63 \%$ \\
$1.0 \mathrm{E}+05$ & 3.9326 & $6.19 \%$ & 2.4221 & $10.56 \%$ \\
\hline $2.5 \mathrm{E}+05$ & 4.8453 & $2.11 \%$ & 2.9278 & $6.51 \%$ \\
\hline $5.0 \mathrm{E}+05$ & 5.6741 & $1.37 \%$ & 3.3795 & $5.66 \%$ \\
\hline $7.5 \mathrm{E}+05$ & 6.2231 & $0.18 \%$ & 3.6753 & $1.70 \%$ \\
\hline $1.0 \mathrm{E}+06$ & 6.6446 & $-0.49 \%$ & 3.9008 & $2.36 \%$ \\
\hline $2.5 \mathrm{E}+06$ & 8.1868 & $-1.06 \%$ & 4.7153 & $-2.01 \%$ \\
\hline $5.0 \mathrm{E}+06$ & 9.5870 & $-1.55 \%$ & 5.4426 & $-3.85 \%$ \\
\hline $7.5 \mathrm{E}+06$ & 10.5147 & $-2.30 \%$ & 5.9191 & $-6.35 \%$ \\
\hline $1.0 \mathrm{E}+07$ & 11.2268 & $-2.17 \%$ & 6.2822 & $-7.31 \%$ \\
\hline Average \% Difference: & $8.14 \%$ & & $10.69 \%$ \\
\hline
\end{tabular}




\section{APPENDIX-B}

NON-UNIFORM GAP THICKNESS DOMED

\section{SKYLIGHT HEATED FROM OUTSIDE}

\section{B.1 Average Nusselt Number}

Table B.1: Average Nusselt number for $\theta_{0}=90^{\circ}$

\begin{tabular}{ccccc}
\hline $\mathbf{G r}$ & $\delta^{\prime}=\mathbf{0 . 5}$ & $\delta^{\prime}=\mathbf{0 . 4}$ & $\delta^{\prime}=\mathbf{0 . 3}$ & $\delta^{\prime}=\mathbf{0 . 2 5}$ \\
\hline $1.00 \mathrm{E}+03$ & 1.0713 & 1.0585 & 1.0498 & 1.0465 \\
$2.50 \mathrm{E}+03$ & 1.1111 & 1.0910 & 1.0721 & 1.0635 \\
\hline $5.00 \mathrm{E}+03$ & 1.2015 & 1.1712 & 1.1324 & 1.1116 \\
\hline $7.50 \mathrm{E}+03$ & 1.2943 & 1.2596 & 1.2051 & 1.1724 \\
$1.00 \mathrm{E}+04$ & 1.3798 & 1.3441 & 1.2795 & 1.2373 \\
\hline $2.50 \mathrm{E}+04$ & 1.7395 & 1.7118 & 1.6335 & 1.5713 \\
\hline $5.00 \mathrm{E}+04$ & 2.0726 & 2.0564 & 1.9777 & 1.9108 \\
$7.50 \mathrm{E}+04$ & 2.2878 & 2.2814 & 2.2037 & 2.1337 \\
\hline $1.00 \mathrm{E}+05$ & 2.4502 & 2.4515 & 2.3767 & 2.3042 \\
\hline $2.50 \mathrm{E}+05$ & 3.0392 & 3.0544 & 2.9924 & 2.9173 \\
\hline $5.00 \mathrm{E}+05$ & 3.5885 & 3.6020 & 3.5319 & 3.4561 \\
\hline $7.50 \mathrm{E}+05$ & 3.9607 & 3.9719 & 3.8924 & 3.8126 \\
\hline $1.00 \mathrm{E}+06$ & 4.2499 & 4.2596 & 4.1718 & 4.0872 \\
\hline $2.50 \mathrm{E}+06$ & 5.3283 & 5.3337 & 5.2204 & 5.0992 \\
\hline $5.00 \mathrm{E}+06$ & 6.3295 & 6.3307 & 6.1957 & 6.0454 \\
\hline $7.50 \mathrm{E}+06$ & 7.0024 & 7.0013 & 6.8500 & 6.6851 \\
$1.00 \mathrm{E}+07$ & 7.5231 & 7.5201 & 7.3561 & 7.1780 \\
\hline
\end{tabular}


Table B.1: Continued

\begin{tabular}{ccccc}
\hline $\mathbf{G r}$ & $\delta^{\prime}=\mathbf{0 . 2}$ & $\delta^{\prime}=\mathbf{0 . 1 5}$ & $\delta^{\prime}=\mathbf{0 . 1}$ & $\delta^{\prime}=\mathbf{0 . 0 5}$ \\
\hline $1.00 \mathrm{E}+03$ & 1.0441 & 1.0420 & 1.0408 & 1.0400 \\
$2.50 \mathrm{E}+03$ & 1.0560 & 1.0494 & 1.0444 & 1.0410 \\
\hline $5.00 \mathrm{E}+03$ & 1.0913 & 1.0724 & 1.0562 & 1.0443 \\
\hline $7.50 \mathrm{E}+03$ & 1.1382 & 1.1046 & 1.0738 & 1.0496 \\
$1.00 \mathrm{E}+04$ & 1.1906 & 1.1421 & 1.0955 & 1.0566 \\
\hline $2.50 \mathrm{E}+04$ & 1.4893 & 1.3845 & 1.2573 & 1.1220 \\
\hline $5.00 \mathrm{E}+04$ & 1.8182 & 1.6901 & 1.5105 & 1.2608 \\
$7.50 \mathrm{E}+04$ & 2.0360 & 1.8986 & 1.7014 & 1.3964 \\
\hline $1.00 \mathrm{E}+05$ & 2.2025 & 2.0581 & 1.8494 & 1.5159 \\
\hline $2.50 \mathrm{E}+05$ & 2.7997 & 2.6361 & 2.3916 & 1.9816 \\
$5.00 \mathrm{E}+05$ & 3.3306 & 3.1310 & 2.8645 & 2.4100 \\
\hline $7.50 \mathrm{E}+05$ & 3.6829 & 3.4700 & 3.1612 & 2.6857 \\
\hline $1.00 \mathrm{E}+06$ & 3.9504 & 3.7358 & 3.3923 & 2.8937 \\
\hline $2.50 \mathrm{E}+06$ & 4.9294 & 4.6715 & 4.3005 & 3.6294 \\
\hline $5.00 \mathrm{E}+06$ & 5.8290 & 5.5203 & 5.0760 & 4.3595 \\
\hline $7.50 \mathrm{E}+06$ & 6.4351 & 6.0969 & 5.5949 & 4.7997 \\
\hline $1.00 \mathrm{E}+07$ & 6.9075 & 6.5419 & 5.9997 & 5.1412 \\
\hline
\end{tabular}


Table B.1: Continued

\begin{tabular}{ccccc}
\hline $\mathbf{G r}$ & $\delta^{\prime}=\mathbf{0 . 0 2 5}$ & $\delta^{\prime}=\mathbf{0 . 0 1 5}$ & $\delta^{\prime}=\mathbf{0 . 0 1}$ & $\delta^{\prime}=\mathbf{0 . 0 0 8}$ \\
\hline $1.00 \mathrm{E}+03$ & 1.0398 & 1.0397 & 1.0398 & 1.0397 \\
$2.50 \mathrm{E}+03$ & 1.0400 & 1.0398 & 1.0398 & 1.0397 \\
\hline $5.00 \mathrm{E}+03$ & 1.0409 & 1.0402 & 1.0400 & 1.0399 \\
$7.50 \mathrm{E}+03$ & 1.0423 & 1.0407 & 1.0402 & 1.0400 \\
$1.00 \mathrm{E}+04$ & 1.0442 & 1.0414 & 1.0405 & 1.0402 \\
\hline $2.50 \mathrm{E}+04$ & 1.0651 & 1.0498 & 1.0444 & 1.0428 \\
\hline $5.00 \mathrm{E}+04$ & 1.1221 & 1.0755 & 1.0572 & 1.0513 \\
$7.50 \mathrm{E}+04$ & 1.1904 & 1.1103 & 1.0758 & 1.0642 \\
\hline $1.00 \mathrm{E}+05$ & 1.2613 & 1.1497 & 1.0984 & 1.0802 \\
\hline $2.50 \mathrm{E}+05$ & 1.6205 & 1.3985 & 1.2647 & 1.2061 \\
$5.00 \mathrm{E}+05$ & 1.9857 & 1.7126 & 1.5135 & 1.4227 \\
\hline $7.50 \mathrm{E}+05$ & 2.2295 & 1.9278 & 1.7003 & 1.5945 \\
\hline $1.00 \mathrm{E}+06$ & 2.4161 & 2.0938 & 1.8454 & 1.7319 \\
\hline $2.50 \mathrm{E}+06$ & 3.0758 & 2.6976 & 2.4179 & 2.2725 \\
\hline $5.00 \mathrm{E}+06$ & 3.6464 & 3.2244 & 2.9074 & 2.7432 \\
\hline $7.50 \mathrm{E}+06$ & 4.0352 & 3.5617 & 3.2226 & 3.0467 \\
\hline $1.00 \mathrm{E}+07$ & 4.3588 & 3.8237 & 3.4574 & 3.2761 \\
\hline
\end{tabular}


Table B.2: Average Nusselt number for $\theta_{0}=45^{\circ}$

\begin{tabular}{ccccc}
\hline $\mathbf{G r}$ & $\delta^{\prime}=\mathbf{0 . 4}$ & $\delta^{\prime}=\mathbf{0 . 3 5}$ & $\delta^{\prime}=\mathbf{0 . 3}$ & $\delta^{\prime}=\mathbf{0 . 2 5}$ \\
\hline $1.00 \mathrm{E}+03$ & 1.0969 & 1.0825 & 1.0701 & 1.0604 \\
$2.50 \mathrm{E}+03$ & 1.1058 & 1.0925 & 1.0808 & 1.0708 \\
$5.00 \mathrm{E}+03$ & 1.1243 & 1.1147 & 1.1060 & 1.0973 \\
$7.50 \mathrm{E}+03$ & 1.1428 & 1.1375 & 1.1334 & 1.1280 \\
$1.00 \mathrm{E}+04$ & 1.1598 & 1.1591 & 1.1600 & 1.1592 \\
$2.50 \mathrm{E}+04$ & 1.2322 & 1.2543 & 1.2843 & 1.3149 \\
$5.00 \mathrm{E}+04$ & 1.2972 & 1.3426 & 1.4057 & 1.4771 \\
$7.50 \mathrm{E}+04$ & 1.3357 & 1.3959 & 1.4816 & 1.5822 \\
$1.00 \mathrm{E}+05$ & 1.3625 & 1.4335 & 1.5365 & 1.6598 \\
$2.50 \mathrm{E}+05$ & 1.4441 & 1.5504 & 1.7153 & 1.9238 \\
$5.00 \mathrm{E}+05$ & 1.5014 & 1.6359 & 1.8561 & 2.1471 \\
$7.50 \mathrm{E}+05$ & 1.5334 & 1.6849 & 1.9416 & 2.2909 \\
$1.00 \mathrm{E}+06$ & 1.5514 & 1.7192 & 2.0040 & 2.3999 \\
$2.50 \mathrm{E}+06$ & 1.6197 & 1.8267 & 2.2124 & 2.7912 \\
$5.00 \mathrm{E}+06$ & 1.6702 & 1.9068 & 2.3807 & 3.1380 \\
$7.50 \mathrm{E}+06$ & 1.6996 & 1.9534 & 2.4839 & 3.3679 \\
$1.00 \mathrm{E}+07$ & 1.7204 & 1.9865 & 2.5594 & 3.5441 \\
\hline & & & &
\end{tabular}


Table B.2: Continued

\begin{tabular}{ccccc}
\hline $\mathbf{G r}$ & $\delta^{\prime}=\mathbf{0 . 2}$ & $\delta^{\prime}=\mathbf{0 . 1 5}$ & $\delta^{\prime}=\mathbf{0 . 1}$ & $\delta^{\prime}=\mathbf{0 . 0 5}$ \\
\hline $1.00 \mathrm{E}+03$ & 1.0527 & 1.0467 & 1.0428 & 1.0404 \\
$2.50 \mathrm{E}+03$ & 1.0618 & 1.0534 & 1.0467 & 1.0417 \\
\hline $5.00 \mathrm{E}+03$ & 1.0866 & 1.0732 & 1.0588 & 1.0458 \\
\hline $7.50 \mathrm{E}+03$ & 1.1177 & 1.1002 & 1.0765 & 1.0522 \\
$1.00 \mathrm{E}+04$ & 1.1511 & 1.1309 & 1.0981 & 1.0606 \\
\hline $2.50 \mathrm{E}+04$ & 1.3327 & 1.3199 & 1.2569 & 1.1377 \\
\hline $5.00 \mathrm{E}+04$ & 1.5357 & 1.5520 & 1.4899 & 1.2981 \\
\hline $7.50 \mathrm{E}+04$ & 1.6709 & 1.7108 & 1.6600 & 1.4454 \\
\hline $1.00 \mathrm{E}+05$ & 1.7721 & 1.8307 & 1.7904 & 1.5690 \\
\hline $2.50 \mathrm{E}+05$ & 2.1235 & 2.2514 & 2.2539 & 2.0279 \\
\hline $5.00 \mathrm{E}+05$ & 2.4307 & 2.6200 & 2.6601 & 2.4388 \\
\hline $7.50 \mathrm{E}+05$ & 2.6342 & 2.8650 & 2.9256 & 2.7023 \\
\hline $1.00 \mathrm{E}+06$ & 2.7912 & 3.0550 & 3.1299 & 2.8989 \\
\hline $2.50 \mathrm{E}+06$ & 3.3713 & 3.7645 & 3.8839 & 3.6506 \\
\hline $5.00 \mathrm{E}+06$ & 3.9010 & 4.4166 & 4.5858 & 4.3170 \\
\hline $7.50 \mathrm{E}+06$ & 4.2589 & 4.8578 & 5.0641 & 4.7571 \\
\hline $1.00 \mathrm{E}+07$ & 4.5363 & 5.2001 & 5.4359 & 5.0974 \\
\hline
\end{tabular}


Table B.2: Continued

\begin{tabular}{ccccc}
\hline $\mathbf{G r}$ & $\delta^{\prime}=\mathbf{0 . 0 2 5}$ & $\delta^{\prime}=\mathbf{0 . 0 1 5}$ & $\delta^{\prime}=\mathbf{0 . 0 1}$ & $\delta^{\prime}=\mathbf{0 . 0 0 8}$ \\
\hline $1.00 \mathrm{E}+03$ & 1.0398 & 1.0396 & 1.0397 & 1.0396 \\
$2.50 \mathrm{E}+03$ & 1.0402 & 1.0399 & 1.0398 & 1.0398 \\
\hline $5.00 \mathrm{E}+03$ & 1.0415 & 1.0404 & 1.0400 & 1.0399 \\
\hline $7.50 \mathrm{E}+03$ & 1.0435 & 1.0412 & 1.0404 & 1.0402 \\
$1.00 \mathrm{E}+04$ & 1.0462 & 1.0422 & 1.0409 & 1.0405 \\
\hline $2.50 \mathrm{E}+04$ & 1.0744 & 1.0541 & 1.0468 & 1.0443 \\
\hline $5.00 \mathrm{E}+04$ & 1.1479 & 1.0892 & 1.0652 & 1.0567 \\
$7.50 \mathrm{E}+04$ & 1.2338 & 1.1352 & 1.0909 & 1.0749 \\
\hline $1.00 \mathrm{E}+05$ & 1.3202 & 1.1866 & 1.1214 & 1.0970 \\
\hline $2.50 \mathrm{E}+05$ & 1.7163 & 1.4886 & 1.3333 & 1.2630 \\
$5.00 \mathrm{E}+05$ & 2.0901 & 1.8266 & 1.6284 & 1.5231 \\
\hline $7.50 \mathrm{E}+05$ & 2.3384 & 2.0511 & 1.8361 & 1.7121 \\
\hline $1.00 \mathrm{E}+06$ & 2.5272 & 2.2242 & 1.9947 & 1.8600 \\
\hline $2.50 \mathrm{E}+06$ & 3.1822 & 2.8419 & 2.5762 & 2.4302 \\
\hline $5.00 \mathrm{E}+06$ & 3.8100 & 3.3774 & 3.0740 & 2.9174 \\
\hline $7.50 \mathrm{E}+06$ & 4.2016 & 3.7629 & 3.3979 & 3.2233 \\
\hline $1.00 \mathrm{E}+07$ & 4.5014 & 4.0407 & 3.6627 & 3.4663 \\
\hline
\end{tabular}


Table B.3: Average Nusselt number for $\theta_{0}=30^{\circ}$

\begin{tabular}{ccccc}
\hline $\mathbf{G r}$ & $\delta^{\prime}=\mathbf{0 . 2}$ & $\delta^{\prime}=\mathbf{0 . 1 5}$ & $\delta^{\prime}=\mathbf{0 . 1}$ & $\delta^{\prime}=\mathbf{0 . 0 5}$ \\
\hline $1.00 \mathrm{E}+03$ & 1.0648 & 1.0538 & 1.0460 & 1.0413 \\
$2.50 \mathrm{E}+03$ & 1.0683 & 1.0574 & 1.0487 & 1.0424 \\
$5.00 \mathrm{E}+03$ & 1.0769 & 1.0672 & 1.0568 & 1.0460 \\
$7.50 \mathrm{E}+03$ & 1.0867 & 1.0785 & 1.0683 & 1.0515 \\
$1.00 \mathrm{E}+04$ & 1.0964 & 1.0929 & 1.0820 & 1.0586 \\
$2.50 \mathrm{E}+04$ & 1.1437 & 1.1679 & 1.1770 & 1.1231 \\
$5.00 \mathrm{E}+04$ & 1.1897 & 1.2540 & 1.3138 & 1.2552 \\
$7.50 \mathrm{E}+04$ & 1.2169 & 1.3102 & 1.4155 & 1.3770 \\
$1.00 \mathrm{E}+05$ & 1.2355 & 1.3507 & 1.4942 & 1.4805 \\
$2.50 \mathrm{E}+05$ & 1.2888 & 1.4767 & 1.7668 & 1.8703 \\
$5.00 \mathrm{E}+05$ & 1.3235 & 1.5670 & 1.9935 & 2.2105 \\
$7.50 \mathrm{E}+05$ & 1.3421 & 1.6185 & 2.1382 & 2.4294 \\
$1.00 \mathrm{E}+06$ & 1.3550 & 1.6548 & 2.2481 & 2.5944 \\
$2.50 \mathrm{E}+06$ & 1.3950 & 1.7706 & 2.6486 & 3.2001 \\
\hline $5.00 \mathrm{E}+06$ & 1.4252 & 1.8597 & 3.0141 & 3.7641 \\
$7.50 \mathrm{E}+06$ & 1.4435 & 1.9127 & 3.2579 & 4.1453 \\
$1.00 \mathrm{E}+07$ & 1.9119 & 1.9509 & 3.4485 & 4.4414 \\
\hline
\end{tabular}


Table B.3: Continued

\begin{tabular}{ccccc}
\hline $\mathbf{G r}$ & $\delta^{\prime}=\mathbf{0 . 0 2 5}$ & $\delta^{\prime}=\mathbf{0 . 0 1 5}$ & $\delta^{\prime}=\mathbf{0 . 0 1}$ & $\delta^{\prime}=\mathbf{0 . 0 0 8}$ \\
\hline $1.00 \mathrm{E}+03$ & 1.0401 & 1.0398 & 1.0395 & 1.0395 \\
$2.50 \mathrm{E}+03$ & 1.0404 & 1.0400 & 1.0396 & 1.0398 \\
\hline $5.00 \mathrm{E}+03$ & 1.0416 & 1.0405 & 1.0398 & 1.0399 \\
\hline $7.50 \mathrm{E}+03$ & 1.0435 & 1.0413 & 1.0405 & 1.0402 \\
$1.00 \mathrm{E}+04$ & 1.0461 & 1.0424 & 1.0410 & 1.0405 \\
\hline $2.50 \mathrm{E}+04$ & 1.0724 & 1.0542 & 1.0472 & 1.0446 \\
\hline $5.00 \mathrm{E}+04$ & 1.1416 & 1.0888 & 1.0660 & 1.0575 \\
$7.50 \mathrm{E}+04$ & 1.2227 & 1.1350 & 1.0922 & 1.0762 \\
\hline $1.00 \mathrm{E}+05$ & 1.3040 & 1.1853 & 1.1231 & 1.0988 \\
\hline $2.50 \mathrm{E}+05$ & 1.6765 & 1.4823 & 1.3372 & 1.2686 \\
$5.00 \mathrm{E}+05$ & 2.0269 & 1.8115 & 1.6309 & 1.5328 \\
\hline $7.50 \mathrm{E}+05$ & 2.2570 & 2.0289 & 1.8353 & 1.7281 \\
\hline $1.00 \mathrm{E}+06$ & 2.4316 & 2.1958 & 1.9913 & 1.8784 \\
\hline $2.50 \mathrm{E}+06$ & 3.0436 & 2.7907 & 2.5611 & 2.4274 \\
\hline $5.00 \mathrm{E}+06$ & 3.6185 & 3.3138 & 3.0501 & 2.9078 \\
\hline $7.50 \mathrm{E}+06$ & 3.9976 & 3.6837 & 3.3681 & 3.2110 \\
\hline $1.00 \mathrm{E}+07$ & 4.2826 & 3.9580 & 3.6200 & 3.4457 \\
\hline & & & & \\
\hline
\end{tabular}




\section{B.2 Curve-Fitting to Coefficient $c$ and $d$}

\section{C, Theta 90}

Rank 1911 Eqn $7002 y=(a+c x) /\left(1+b x+d x^{2}\right)$

$r^{2}=0.996210108$ DF Adj $r^{2}=0.994044456$ FitStdE $r r=0.0031189518$ Fstat $=700.95943$

$a=0.036123749 b=10.375973$

$c=1.7382769 d=-2.3399761$

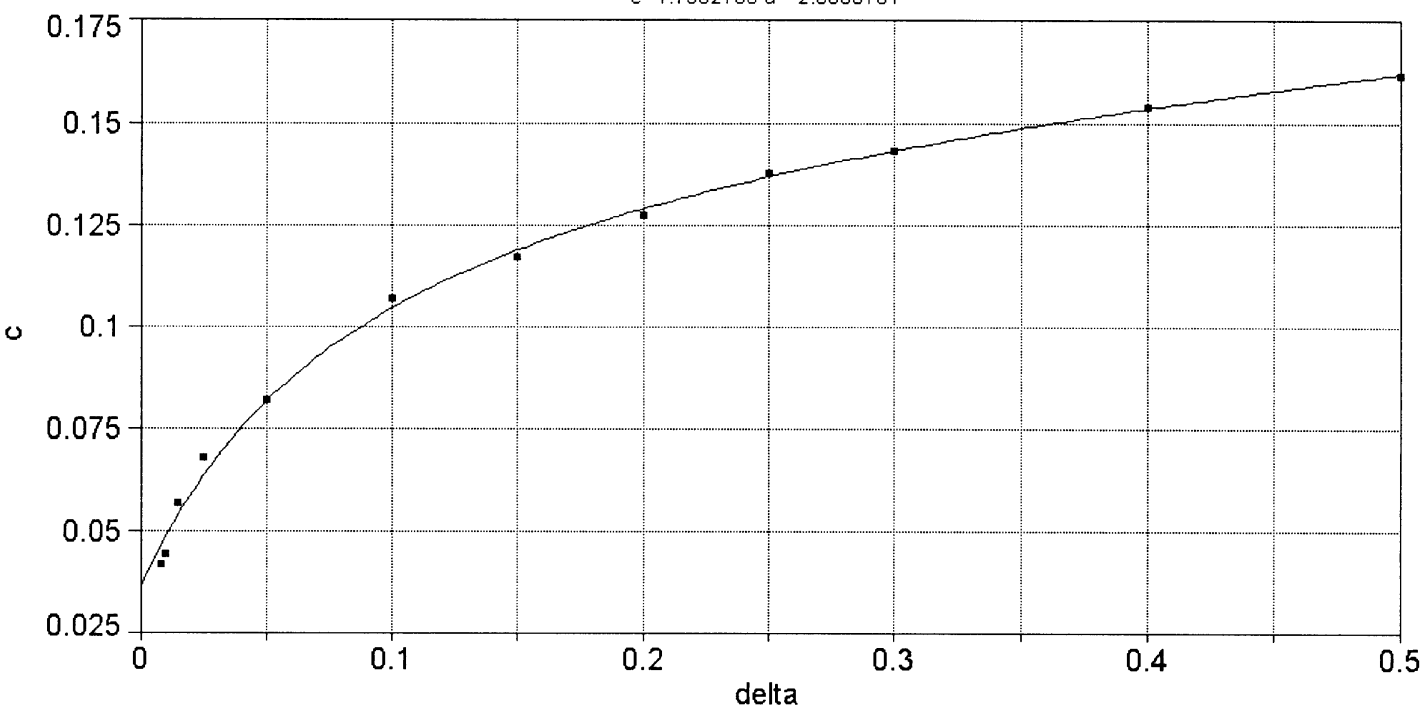

Figure B.1: Curve-fitting to coefficient $c$ for $\theta_{0}=90^{\circ}$

d, Theta90

Rank 2457 Eqn $8010 y=a+b x c$ [Power]

$r^{2}=0.962122484$ DF Adj $r^{2}=0.947918415$ FitStdErr $=0.00945463288$ Fstat $=114.303988$ $a=2.3030483 b=-1.3436489$

$c=0.023004017$

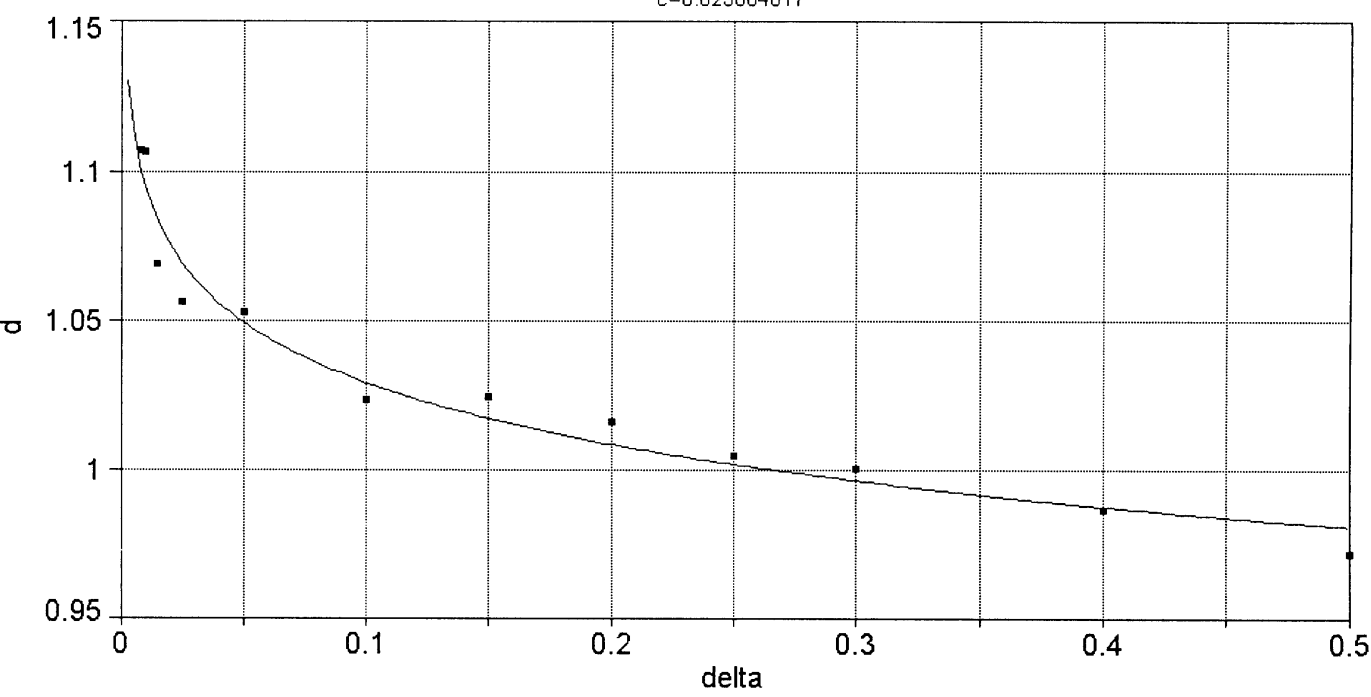

Figure B.2: Curve-fitting to coefficient $d$ for $\theta_{\circ}=90^{\circ}$ 
Table B.4: Coefficient $c$ and $d$ for $\theta_{0}=90^{\circ}$

\begin{tabular}{ccccccc}
\hline $\boldsymbol{\delta}$ & $\boldsymbol{c}$ & $\begin{array}{c}\boldsymbol{c} \\
\text { Curve- } \\
\text { Fitting }\end{array}$ & $\begin{array}{c}\% \\
\text { Difference }\end{array}$ & $\boldsymbol{d}$ & $\begin{array}{c}\boldsymbol{d} \\
\text { Curve- } \\
\text { Fitting }\end{array}$ & $\begin{array}{c}\text { \% } \\
\text { Difference }\end{array}$ \\
\hline 0.500 & 0.1614 & 0.16157 & $-0.10 \%$ & 0.9716 & 0.98065 & $-0.93 \%$ \\
0.400 & 0.1535 & 0.15315 & $0.23 \%$ & 0.9863 & 0.98742 & $-0.11 \%$ \\
0.300 & 0.1429 & 0.14290 & $0.00 \%$ & 1.0003 & 0.99610 & $0.42 \%$ \\
0.250 & 0.1374 & 0.13652 & $0.64 \%$ & 1.0044 & 1.00157 & $0.28 \%$ \\
0.200 & 0.1272 & 0.12872 & $-1.19 \%$ & 1.0157 & 1.00824 & $0.73 \%$ \\
0.150 & 0.1167 & 0.11857 & $-1.60 \%$ & 1.0239 & 1.01678 & $0.70 \%$ \\
0.100 & 0.1067 & 0.10424 & $2.31 \%$ & 1.0233 & 1.02872 & $-0.53 \%$ \\
0.050 & 0.0818 & 0.08132 & $0.58 \%$ & 1.0525 & 1.04888 & $0.34 \%$ \\
0.025 & 0.0678 & 0.06326 & $6.69 \%$ & 1.0558 & 1.06872 & $-1.22 \%$ \\
0.015 & 0.0567 & 0.05385 & $5.03 \%$ & 1.0688 & 1.08314 & $-1.34 \%$ \\
0.010 & 0.0444 & 0.04849 & $-9.20 \%$ & 1.1063 & 1.09446 & $1.07 \%$ \\
0.008 & 0.0418 & 0.04620 & $-10.53 \%$ & 1.1065 & 1.10065 & $0.53 \%$
\end{tabular}


C, Theta 45

Rank 16 Eqn $6002 y=a+b x+c x^{2}+d x^{3}+e x^{4}+f x^{5}$

$r^{2}=0.999569876$ DF Adj $r^{2}=0.999053727$ FitStdErr $=0.00632038832$ Fstat $=2788.69203$

$a=0.04348738 b=0.97983866 c=1.4992258$

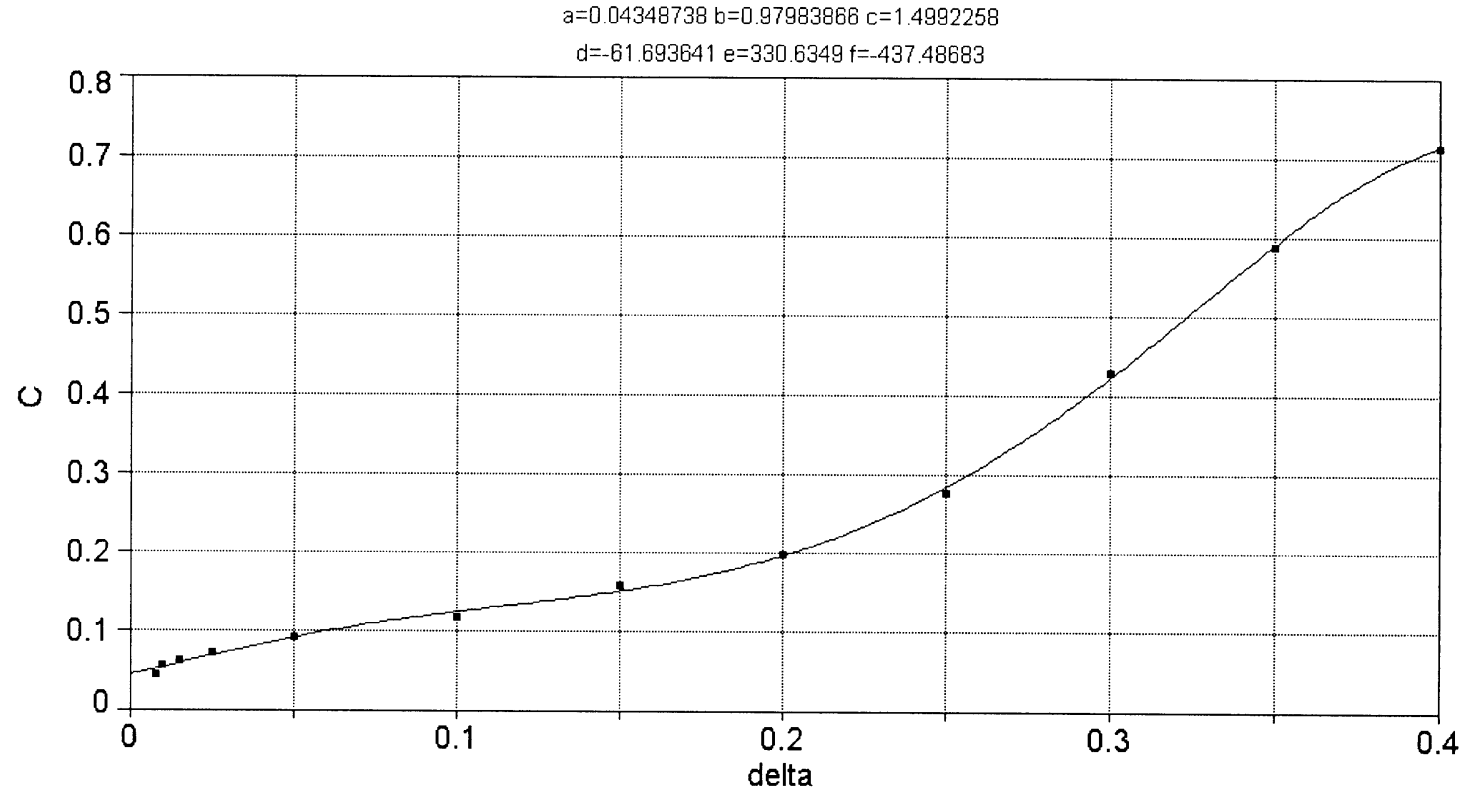

Figure B.3: Curve-fitting to coefficient $c$ for $\theta_{0}=45^{\circ}$

\section{d, Theta 45}

Rank 11 Eqn $6003 y=a+b x+c x^{2}+d x^{3}+e x^{4}+f x^{5}+g x^{6}$

$r^{2}=0.99973005$ DF Adj $r^{2}=0.999100166$ Fit $S t d E r r=0.00815375525$ Fstat $=2468.92483$ $a=1.0565473 b=0.7546041 c=-41.676863 d=406.43125$

$e=-1965.2146 f=4220.9736 g=-3268.143$

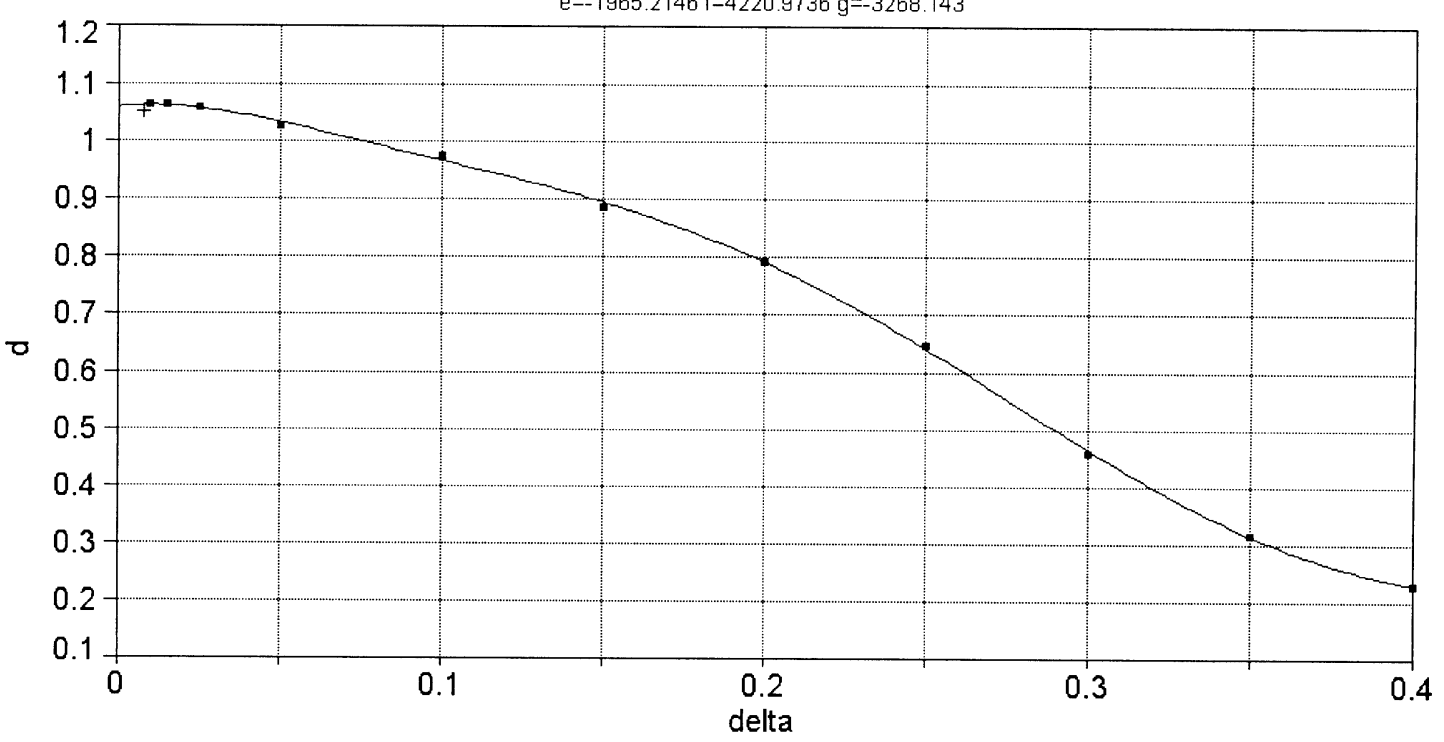

Figure B.4: Curve-fitting to coefficient $d$ for $\theta_{0}=45^{\circ}$ 
Table B.5: Coefficient $c$ and $d$ for $\theta_{0}=45^{\circ}$

\begin{tabular}{ccccccc}
\hline $\boldsymbol{\delta}$ & $\boldsymbol{c}$ & $\begin{array}{c}\boldsymbol{c} \\
\text { Curve- } \\
\text { Fitting }\end{array}$ & $\begin{array}{c}\% \\
\text { Difference }\end{array}$ & $\boldsymbol{d}$ & $\begin{array}{c}\boldsymbol{d} \\
\text { Curve- } \\
\text { Fitting }\end{array}$ & $\begin{array}{c}\% \\
\text { Difference }\end{array}$ \\
\hline 0.400 & 0.7116 & 0.71129 & $0.04 \%$ & 0.2282 & 0.2287 & $-0.20 \%$ \\
0.350 & 0.5866 & 0.58880 & $-0.37 \%$ & 0.3148 & 0.3121 & $0.85 \%$ \\
0.300 & 0.4277 & 0.42169 & $1.40 \%$ & 0.4558 & 0.4619 & $-1.34 \%$ \\
0.250 & 0.2754 & 0.28249 & $-2.58 \%$ & 0.6439 & 0.6384 & $0.85 \%$ \\
0.200 & 0.1959 & 0.19490 & $0.51 \%$ & 0.7909 & 0.7891 & $0.23 \%$ \\
0.150 & 0.1565 & 0.15014 & $4.06 \%$ & 0.8832 & 0.8921 & $-1.01 \%$ \\
0.100 & 0.1172 & 0.12346 & $-5.34 \%$ & 0.9730 & 0.9641 & $0.92 \%$ \\
0.050 & 0.0905 & 0.09045 & $0.06 \%$ & 1.0247 & 1.0299 & $-0.50 \%$ \\
0.025 & 0.0706 & 0.06808 & $3.57 \%$ & 1.0563 & 1.0550 & $0.12 \%$ \\
0.015 & 0.0618 & 0.05833 & $5.61 \%$ & 1.0602 & 1.0598 & $0.04 \%$ \\
0.010 & 0.0558 & 0.05338 & $4.34 \%$ & 1.0603 & 1.0603 & $0.00 \%$ \\
0.008 & 0.0448 & 0.05139 & $-14.71 \%$ & 1.0489 & 1.0601 & $-1.07 \%$
\end{tabular}


Rank 1545 Eqn $2040 y=a+b x+c x^{2}+d x^{3}$

$r^{2}=1$ DF Adj $r^{2}=1$ FitStdE $r=4.71216092 \mathrm{e}-08 \quad$ Fstat $=8.22311273 \mathrm{e}+13$

$a=0.5328 b=-15.555$

$c=160.46 d=-381.6$

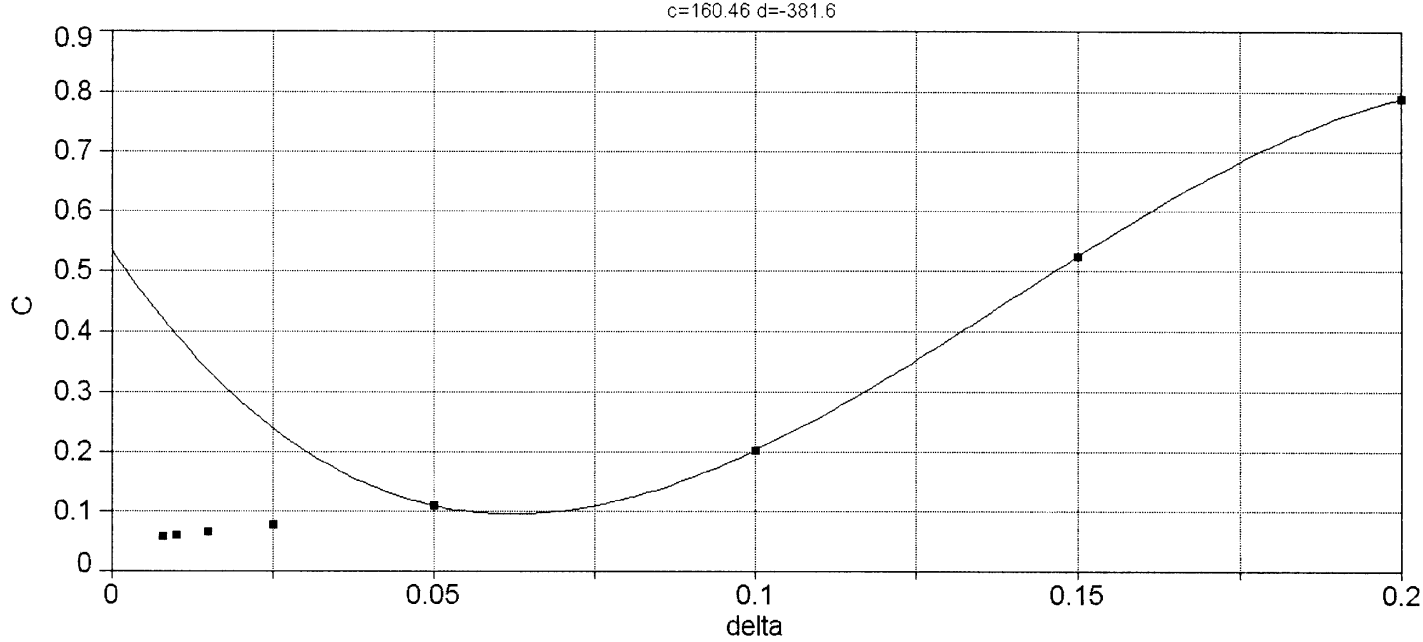

Figure B.5: Curve-fitting to coefficient $c$ for $\theta_{0}=30^{\circ}$ when $\delta^{\prime} \geq 0.1$

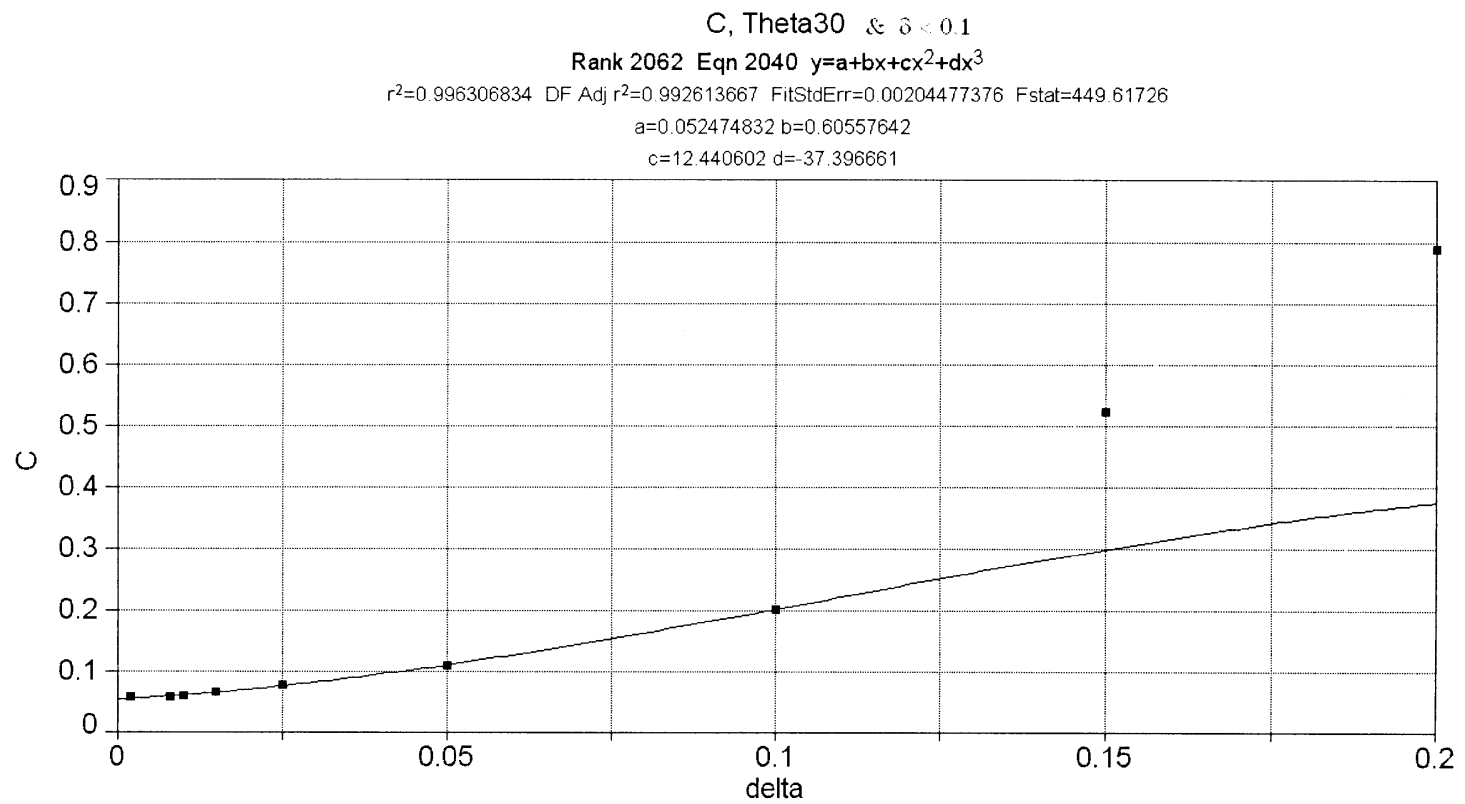

Figure B.6: Curve-fitting to coefficient $c$ for $\theta_{0}=30^{\circ}$ when $\delta^{\prime}<0.1$ 
d, Theta $30 \& \delta^{\prime} \leqslant 0.1$

Rank 2315 Eqn $7002 y=(a+c x) /\left(1+b x+d x^{2}\right)$

$r^{2}=0.998543363$ DF Adj $r^{2}=0.99660118$ FitStdE $r r=0.00426854846 \quad$ Fstat $=914.017135$ $a=1.0445066 \quad b=1.8845856$

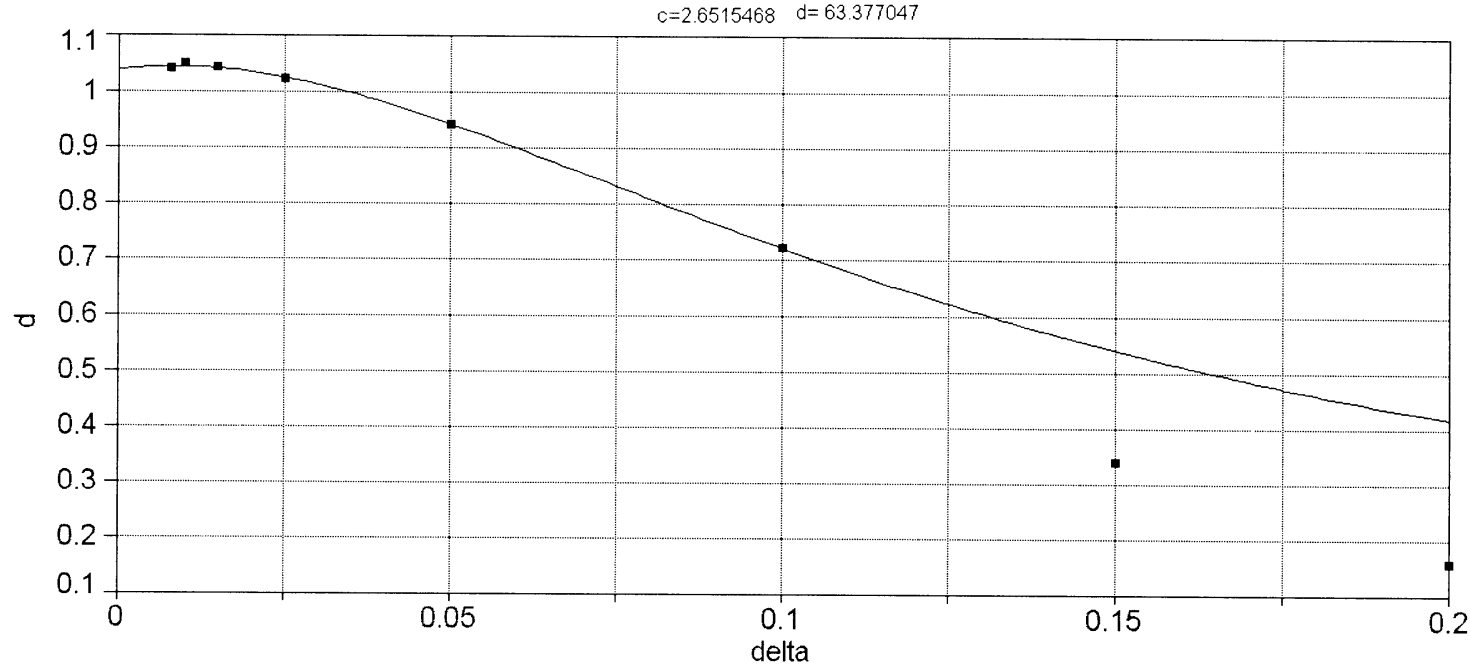

Figure B.7: Curve-fitting to coefficient $d$ for $\theta_{0}=30^{\circ}$ when $\delta^{\prime} \leq 0.1$

d, Theta 30 \& $\delta \geqslant 0.1$

Rank 399 Eqn $7002 y=(a+c x) /\left(1+b x+d x^{2}\right)$

$r^{2}=1$ DF Adj $r^{2}=1$ FitStdE $r=7.27328654 \mathrm{e}-15$ Fstat $=4.75988445 \mathrm{e}+27$

$a=0.61048585 b=-13.329955$

$c=-1.1018624 \mathrm{~d}=102.9113$

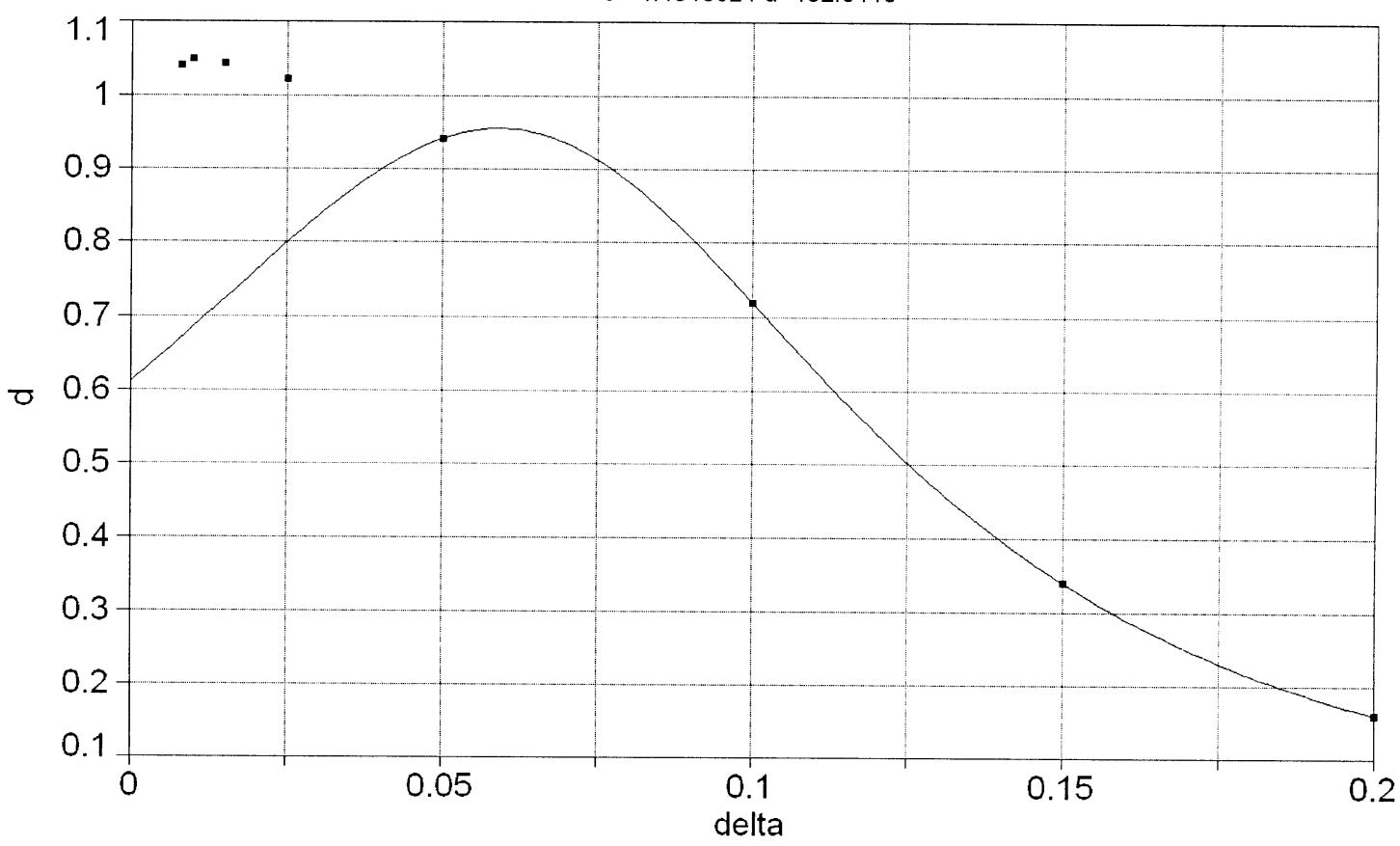

Figure B.8: Curve-fitting to coefficient $d$ for $\theta_{0}=30^{\circ}$ when $\delta^{\prime} \geq 0.1$ 
Table B.6: Coefficient $c$ and $d$ for $\theta_{0}=30^{\circ}$

\begin{tabular}{ccccccc}
\hline$\delta$ & $\boldsymbol{c}$ & $\begin{array}{c}c \\
\text { Curve- } \\
\text { Fitting }\end{array}$ & $\begin{array}{c}\text { \% } \\
\text { Difference }\end{array}$ & $\boldsymbol{d}$ & $\begin{array}{c}\boldsymbol{d} \\
\text { Curve- } \\
\text { Fitting }\end{array}$ & $\begin{array}{c}\% \\
\text { Difference }\end{array}$ \\
\hline 0.200 & 0.7874 & 0.78740 & $0.00 \%$ & 0.1592 & 0.1592 & $0.00 \%$ \\
0.150 & 0.5220 & 0.52200 & $0.00 \%$ & 0.3383 & 0.3383 & $0.00 \%$ \\
0.100 & 0.2003 & 0.20030 & $0.00 \%$ & 0.7187 & 0.7187 & $0.00 \%$ \\
0.050 & 0.1085 & 0.10918 & $-0.63 \%$ & 0.9401 & 0.9397 & $0.05 \%$ \\
0.025 & 0.0769 & 0.07481 & $2.72 \%$ & 1.0205 & 1.0221 & $-0.16 \%$ \\
0.015 & 0.0650 & 0.06423 & $1.18 \%$ & 1.0419 & 1.0400 & $0.18 \%$ \\
0.010 & 0.0581 & 0.05974 & $-2.82 \%$ & 1.0476 & 1.0447 & $0.28 \%$ \\
0.008 & 0.0569 & 0.05810 & $-2.10 \%$ & 1.0391 & 1.0457 & $-0.64 \%$ \\
\hline
\end{tabular}




\section{B.3 Curve-Fitting to The Nusselt Number}

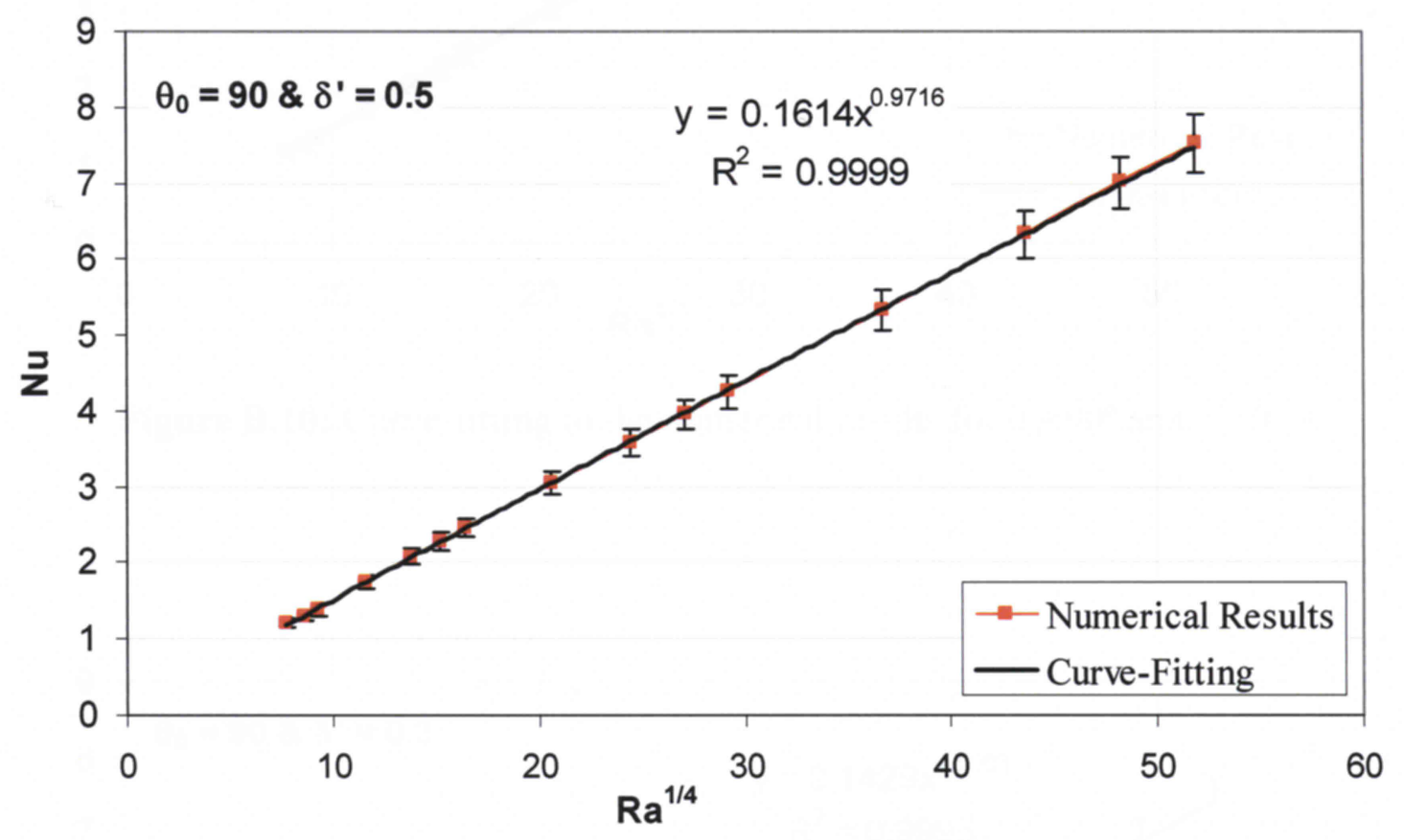

Figure B.9: Curve-fitting to the numerical results for $\theta_{0}=90^{\circ}$ and $\delta '=0.5$ 


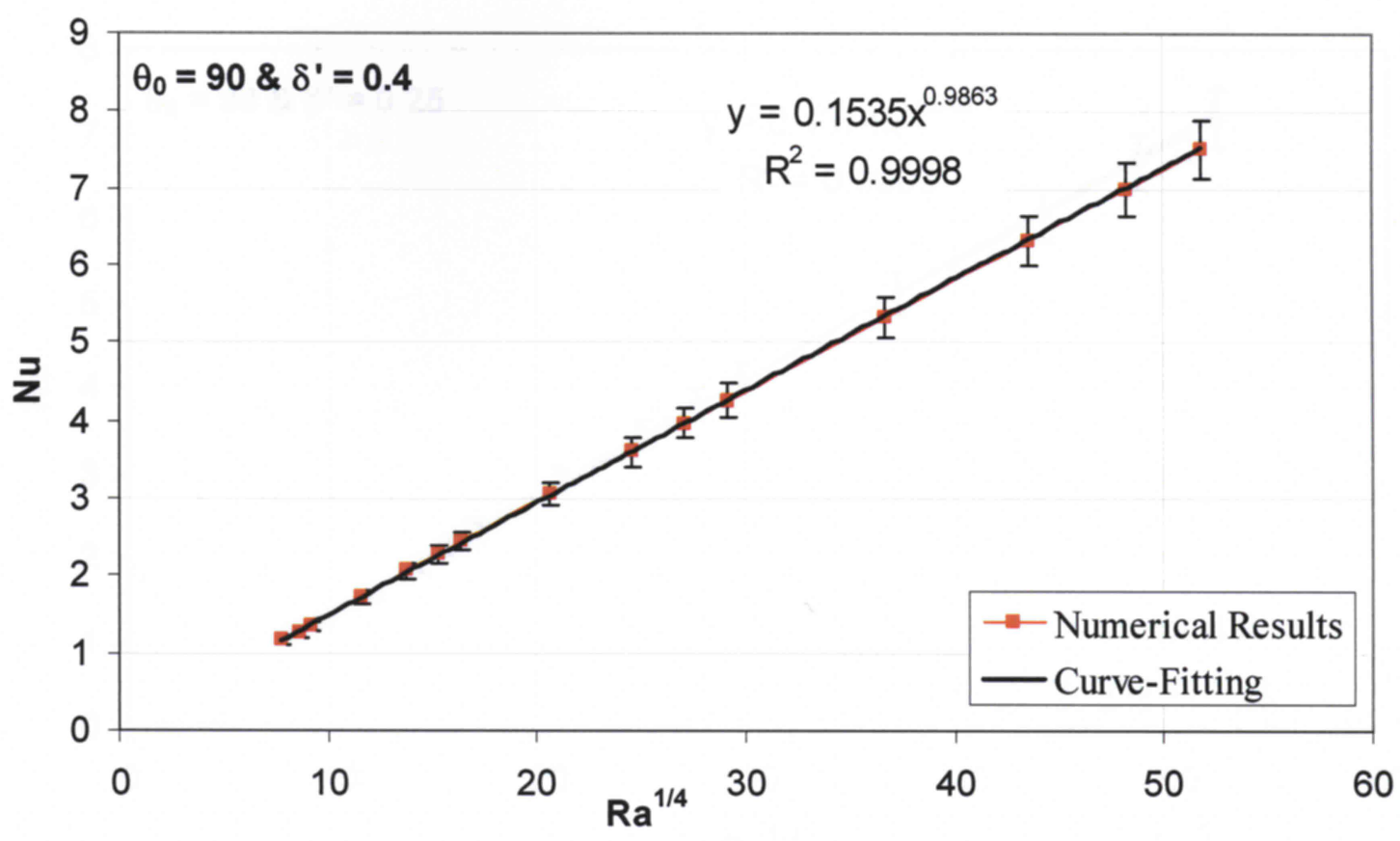

Figure B.10: Curve-fitting to the numerical results for $\theta_{0}=90^{\circ}$ and $\delta^{\prime}=0.4$

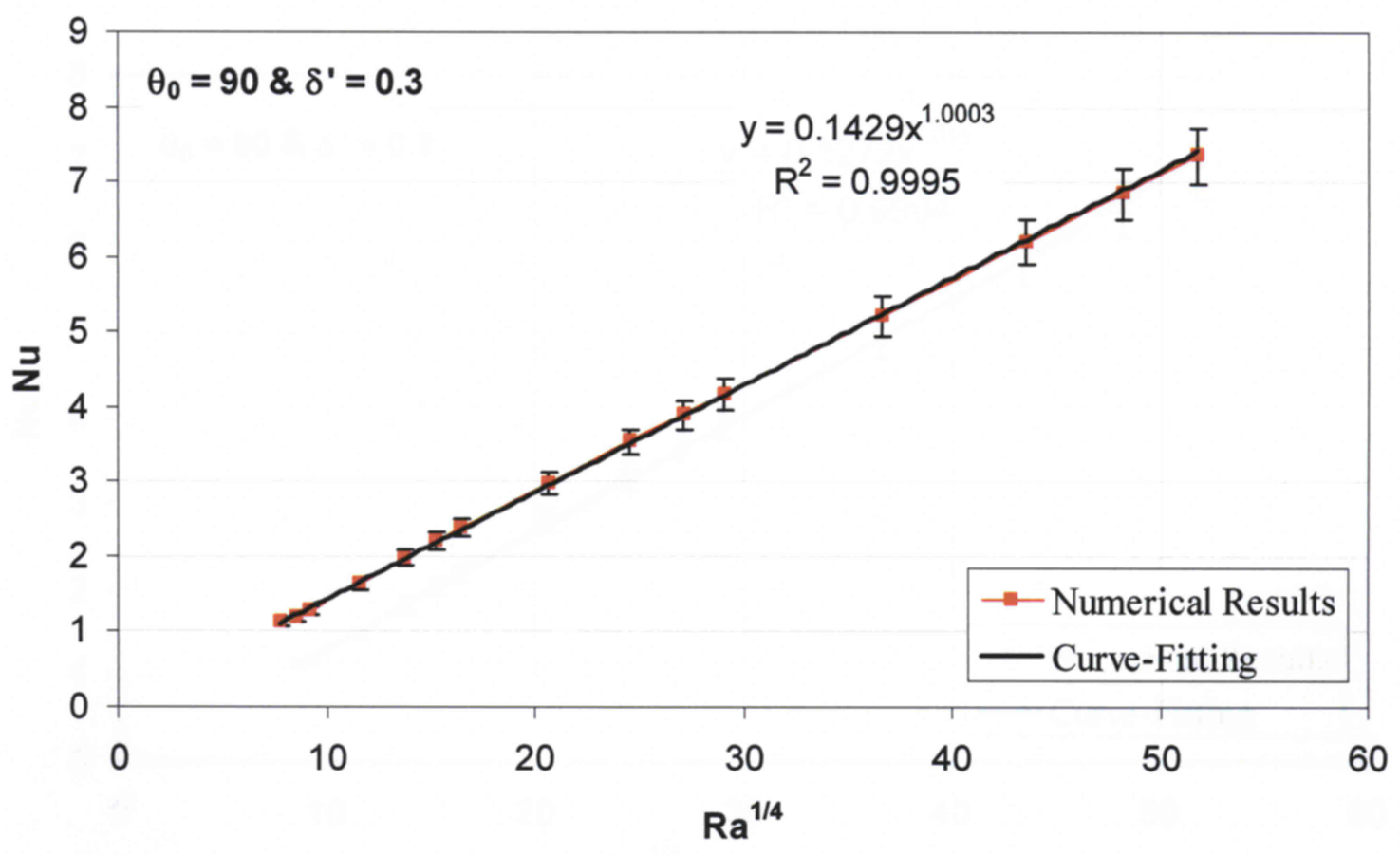

Figure B.11: Curve-fitting to the numerical results for $\theta_{0}=90^{\circ}$ and $\delta^{\prime}=0.3$ 


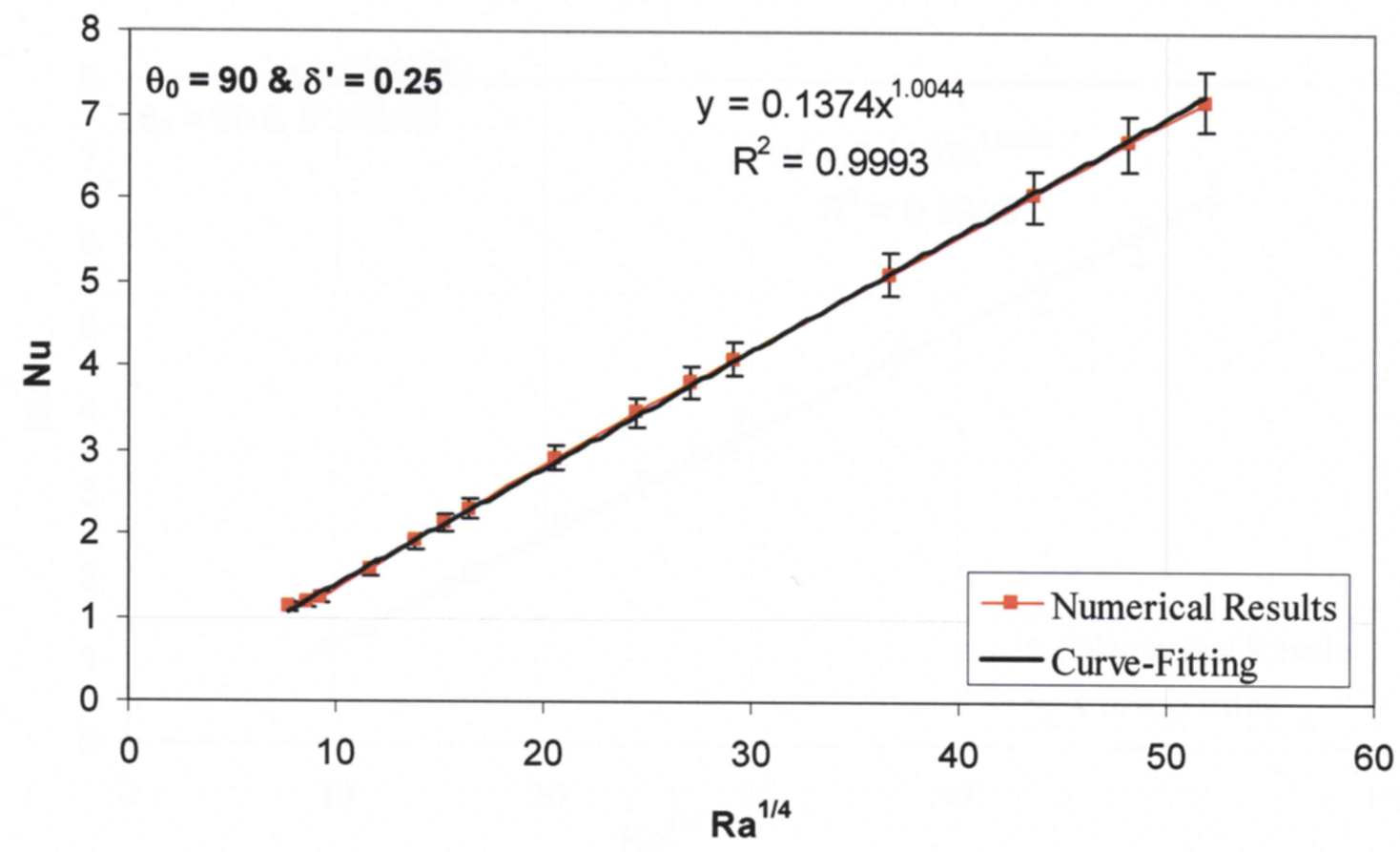

Figure B.12: Curve-fitting to the numerical results for $\theta_{0}=90^{\circ}$ and $\delta^{\prime}=0.25$

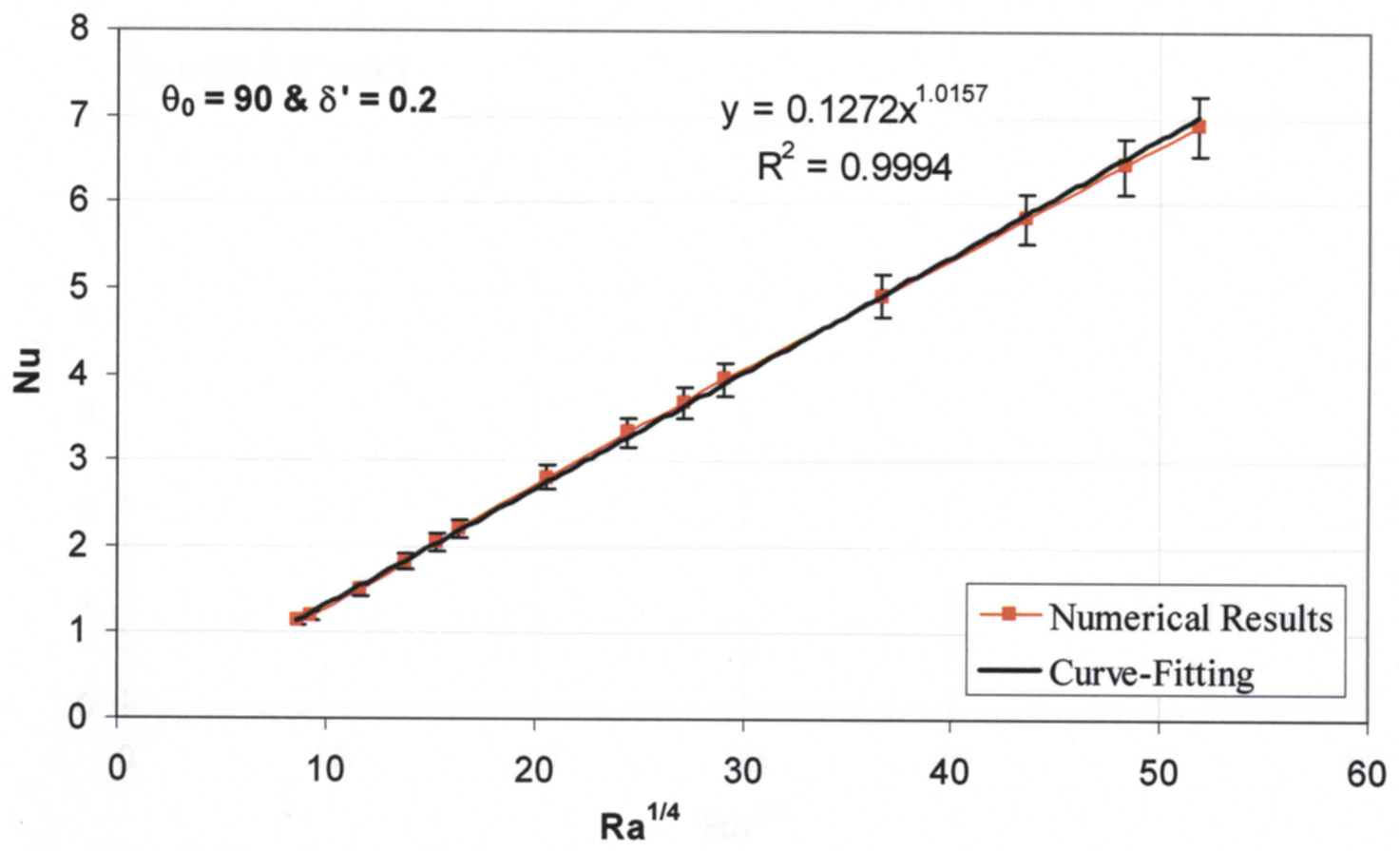

Figure B.13: Curve-fitting to the numerical results for $\theta_{0}=90^{\circ}$ and $\delta^{\prime}=0.2$ 


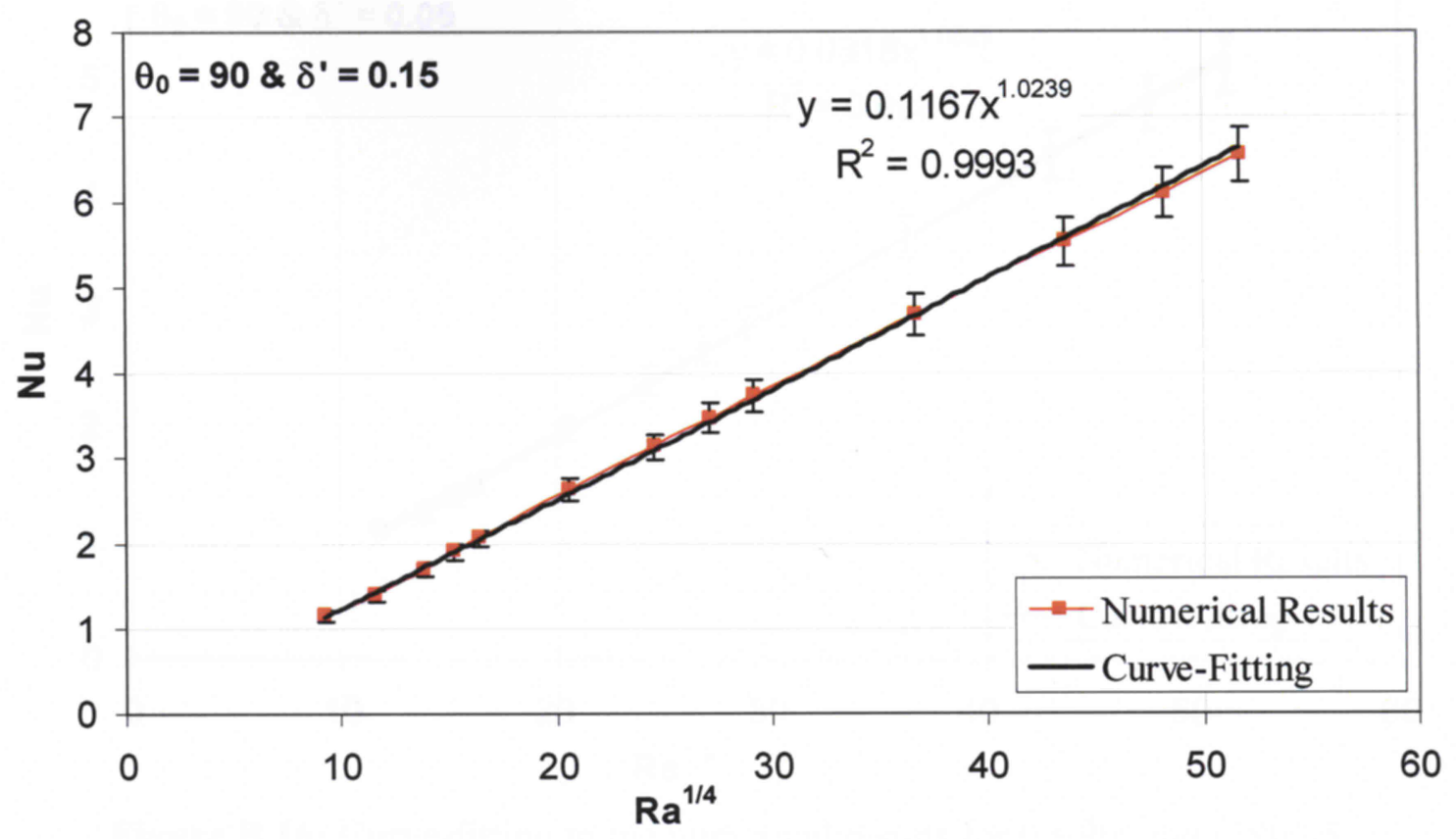

Figure B.14: Curve-fitting to the numerical results for $\theta_{0}=90^{\circ}$ and $\delta^{\prime}=0.15$

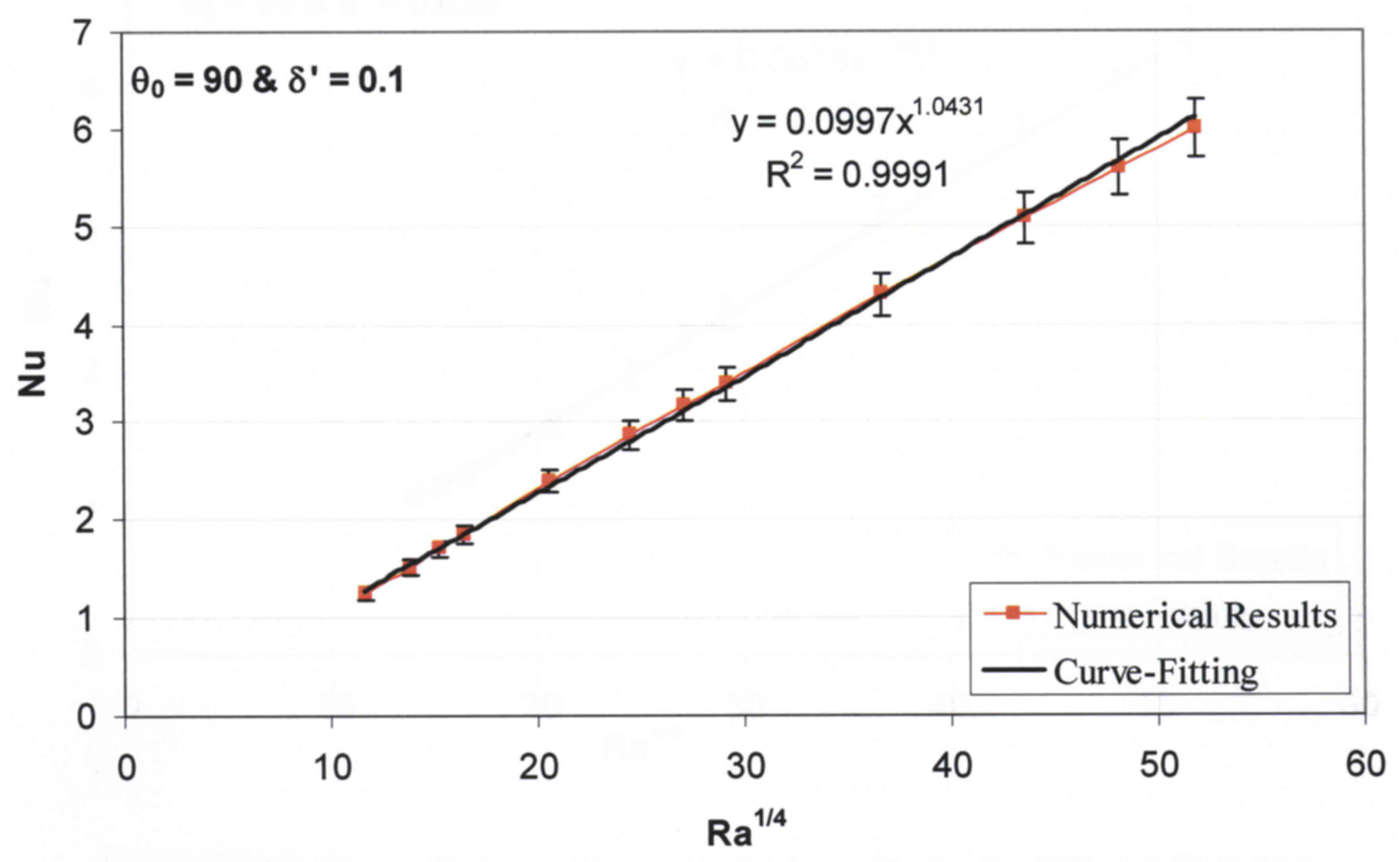

Figure B.15: Curve-fitting to the numerical results for $\theta_{0}=90^{\circ}$ and $\delta^{\prime}=0.1$ 


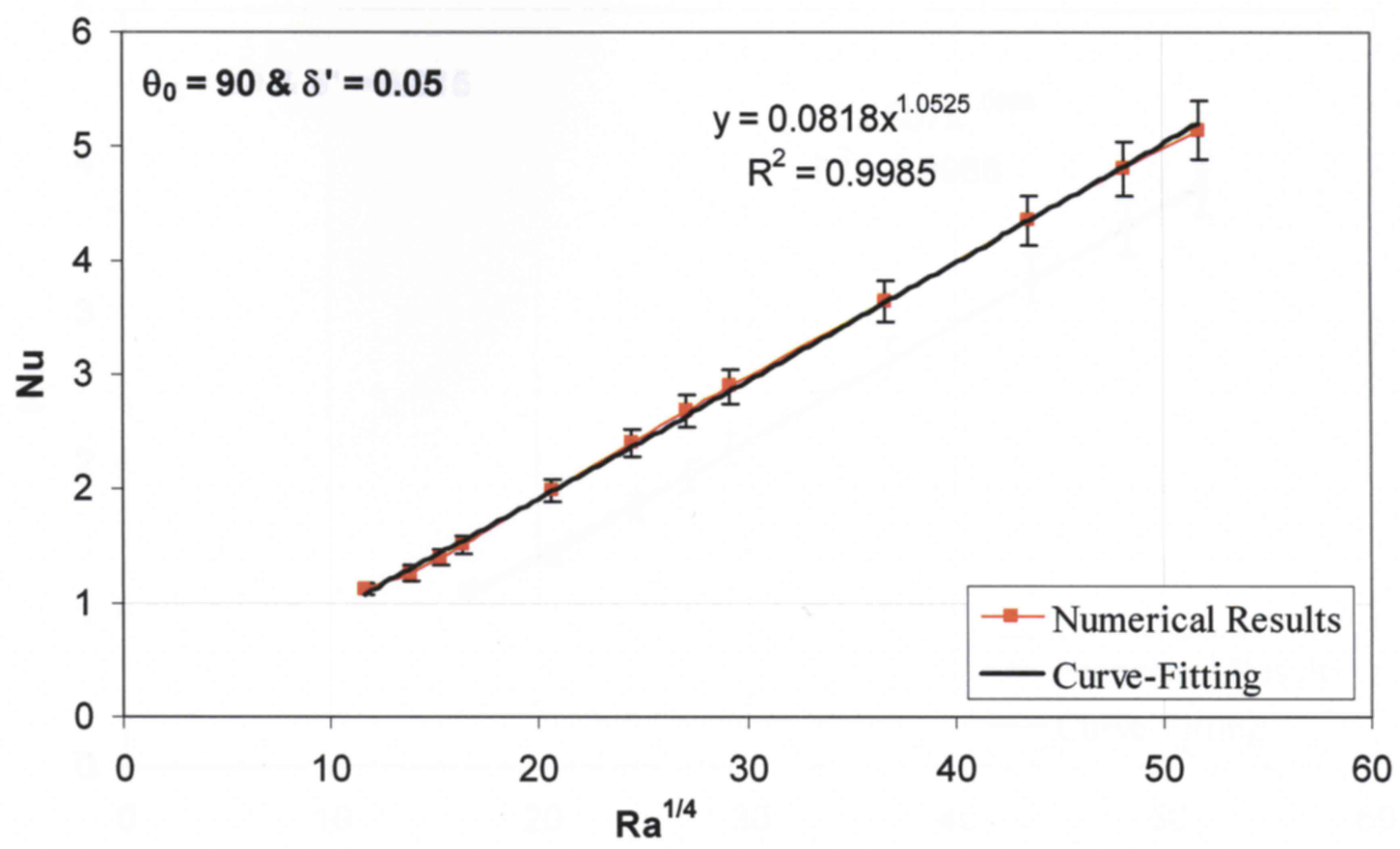

Figure B.16: Curve-fitting to the numerical results for $\theta_{0}=90^{\circ}$ and $\delta^{\prime}=0.05$

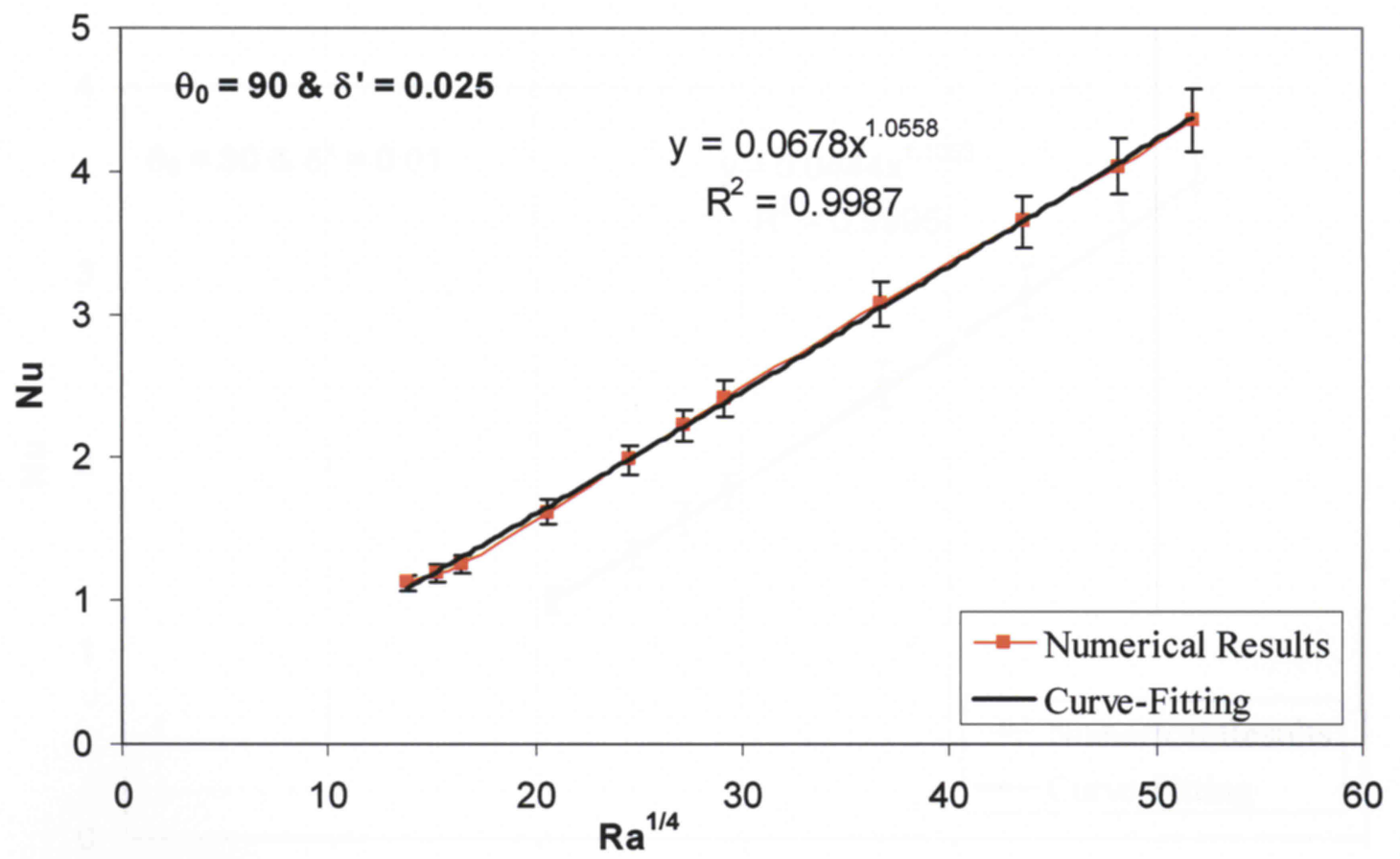

Figure B.17: Curve-fitting to the numerical results for $\theta_{0}=90^{\circ}$ and $\delta^{\prime}=0.025$ 


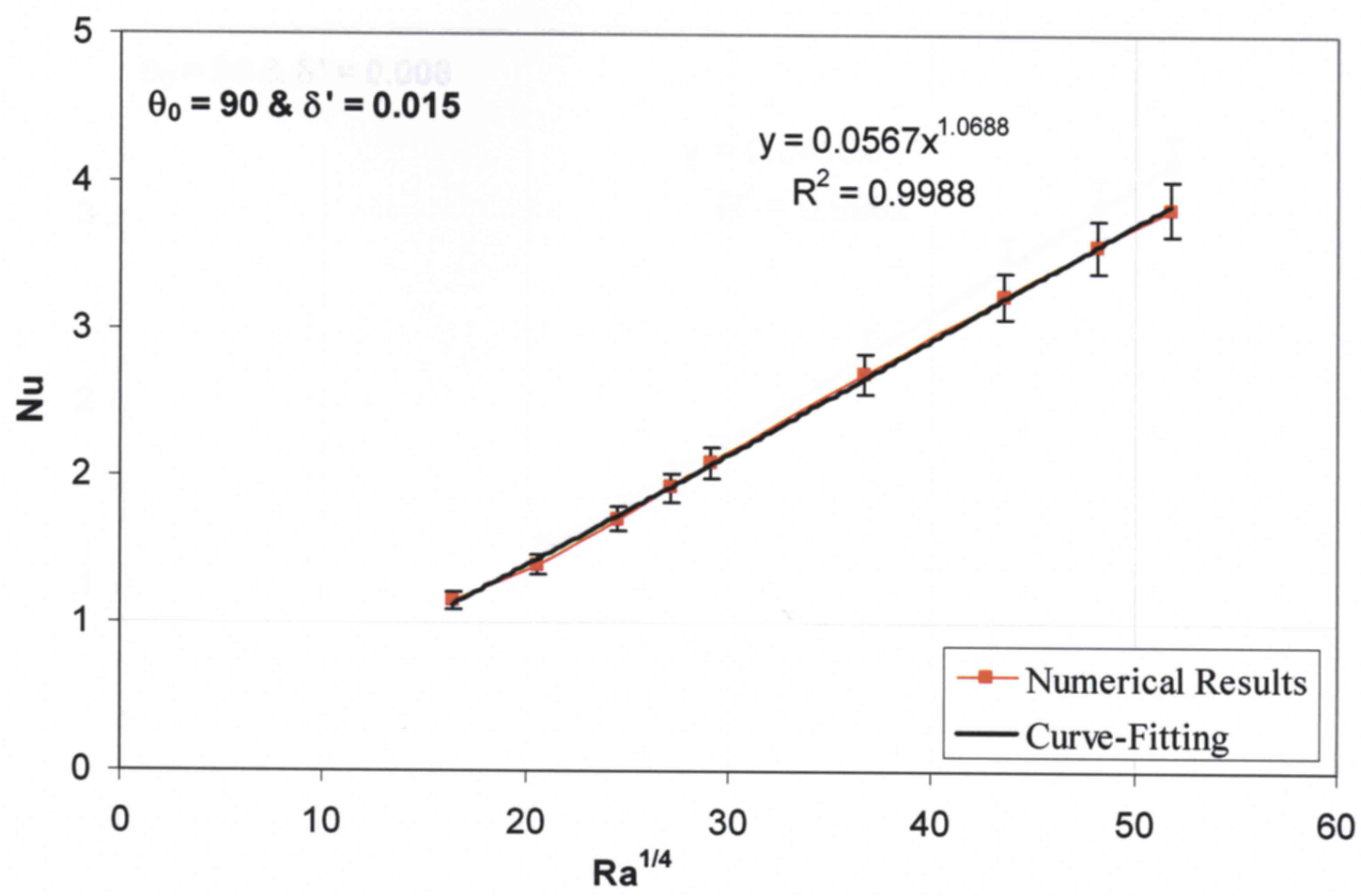

Figure B.18: Curve-fitting to the numerical results for $\theta_{0}=90^{\circ}$ and $\delta^{\prime}=0.015$

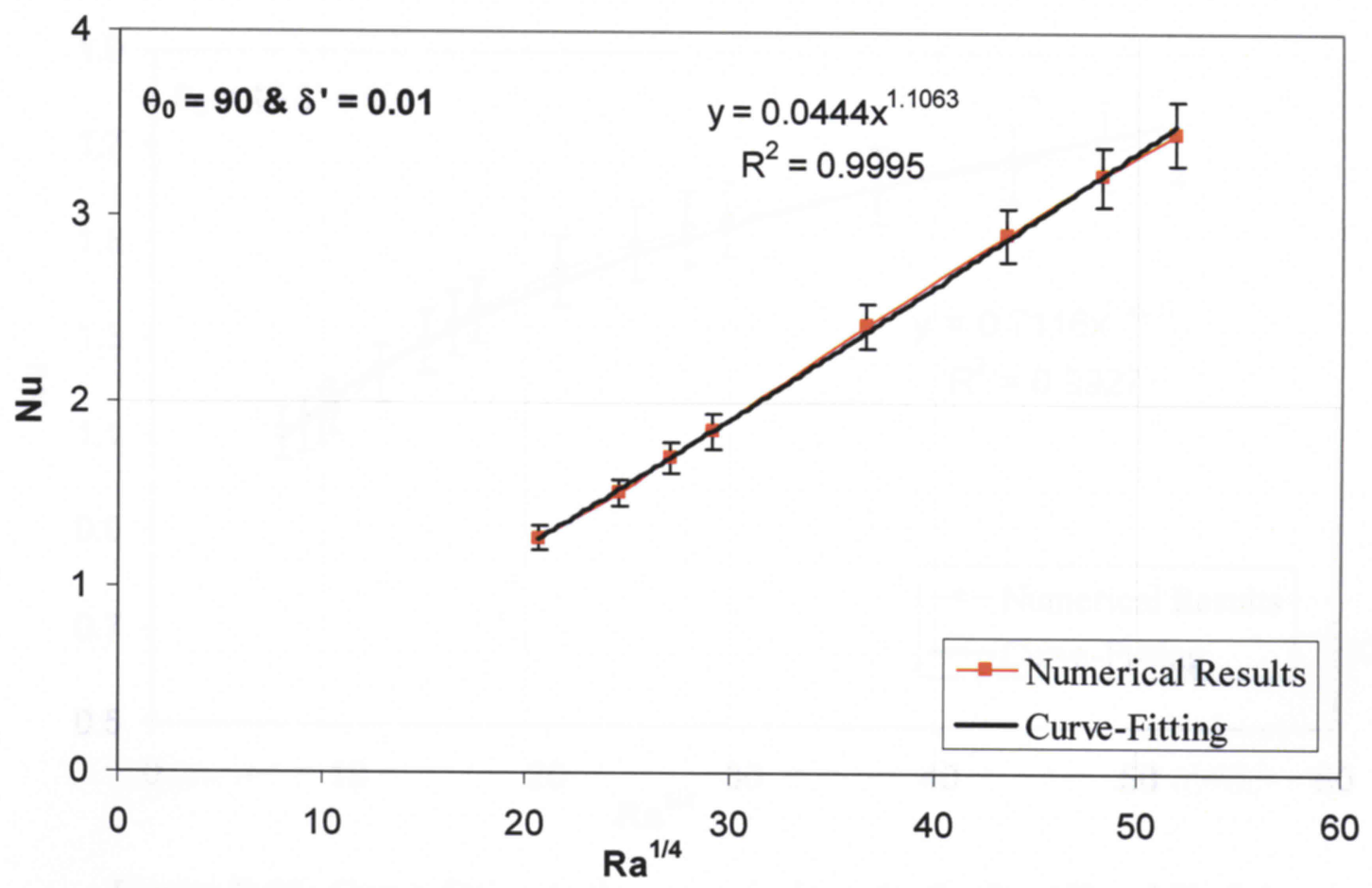

Figure B.19: Curve-fitting to the numerical results for $\theta_{0}=90^{\circ}$ and $\delta^{\prime}=0.01$ 


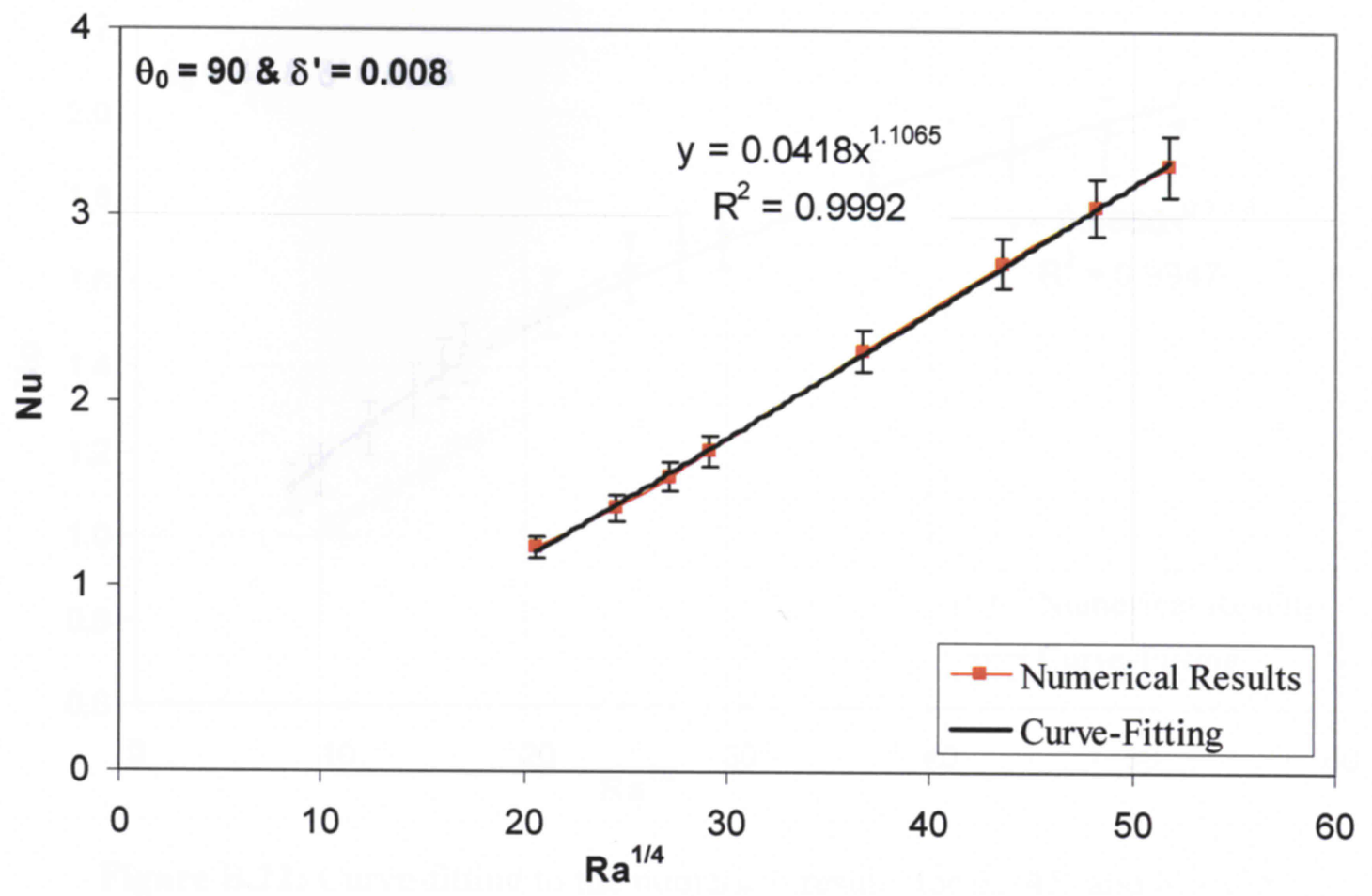

Figure B.20: Curve-fitting to the numerical results for $\theta_{0}=90^{\circ}$ and $\delta^{\prime}=0.008$

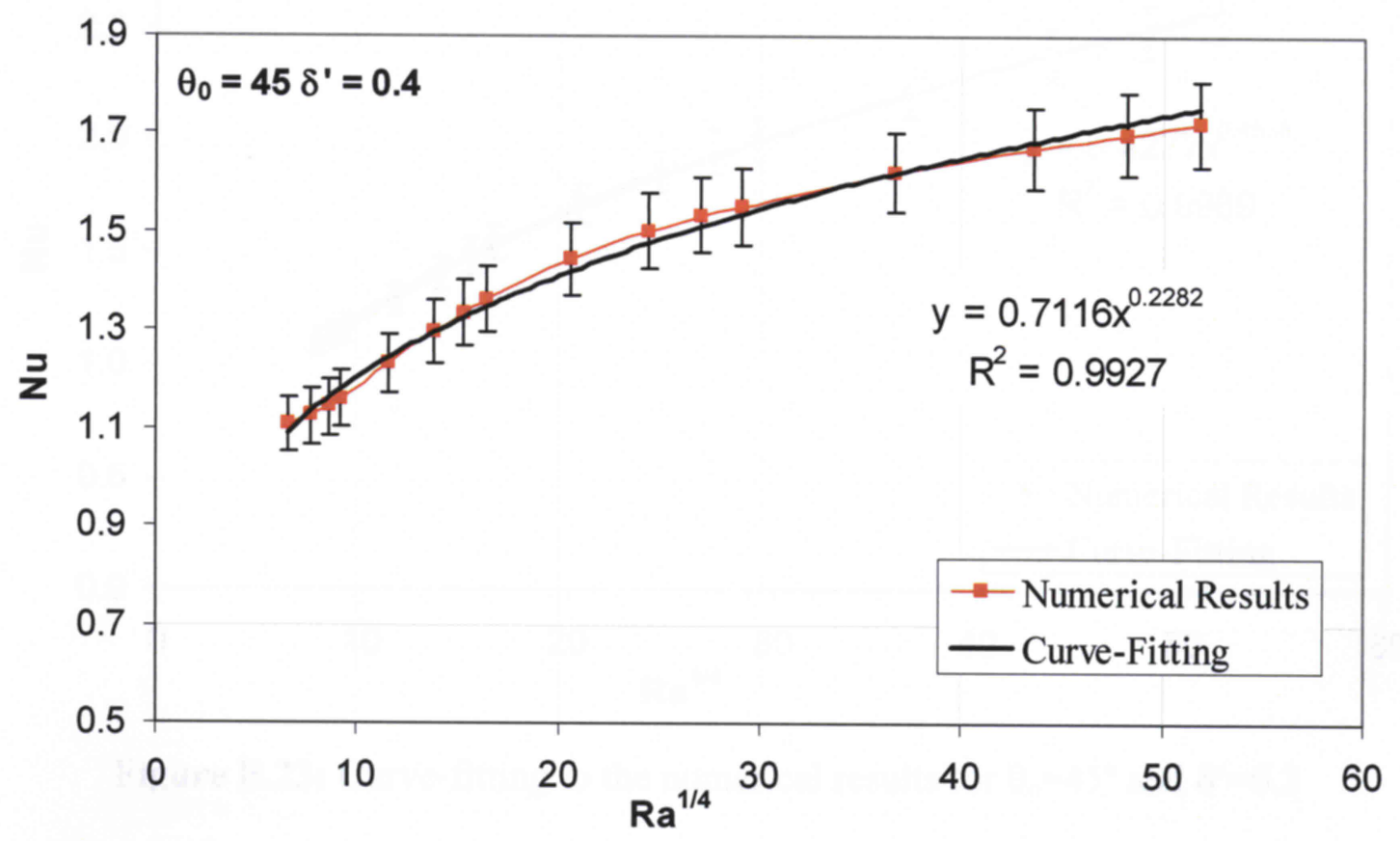

Figure B.21: Curve-fitting to the numerical results for $\theta_{0}=45^{\circ}$ and $\delta^{\prime}=0.4$ 


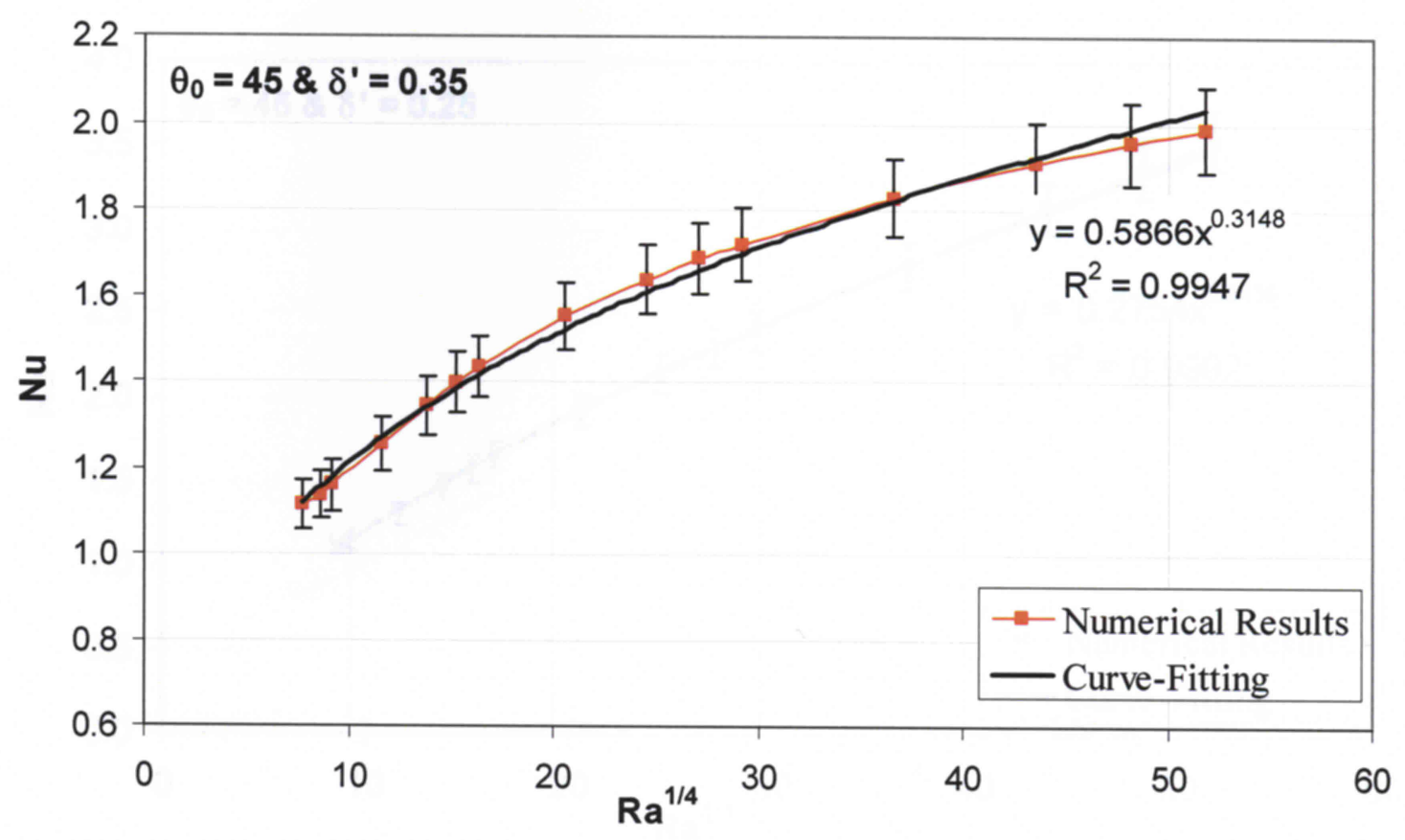

Figure B.22: Curve-fitting to the numerical results for $\theta_{0}=45^{\circ}$ and $\delta^{\prime}=0.35$



Figure B.23: Curve-fitting to the numerical results for $\theta_{0}=45^{\circ}$ and $\delta^{\prime}=0.3$ 


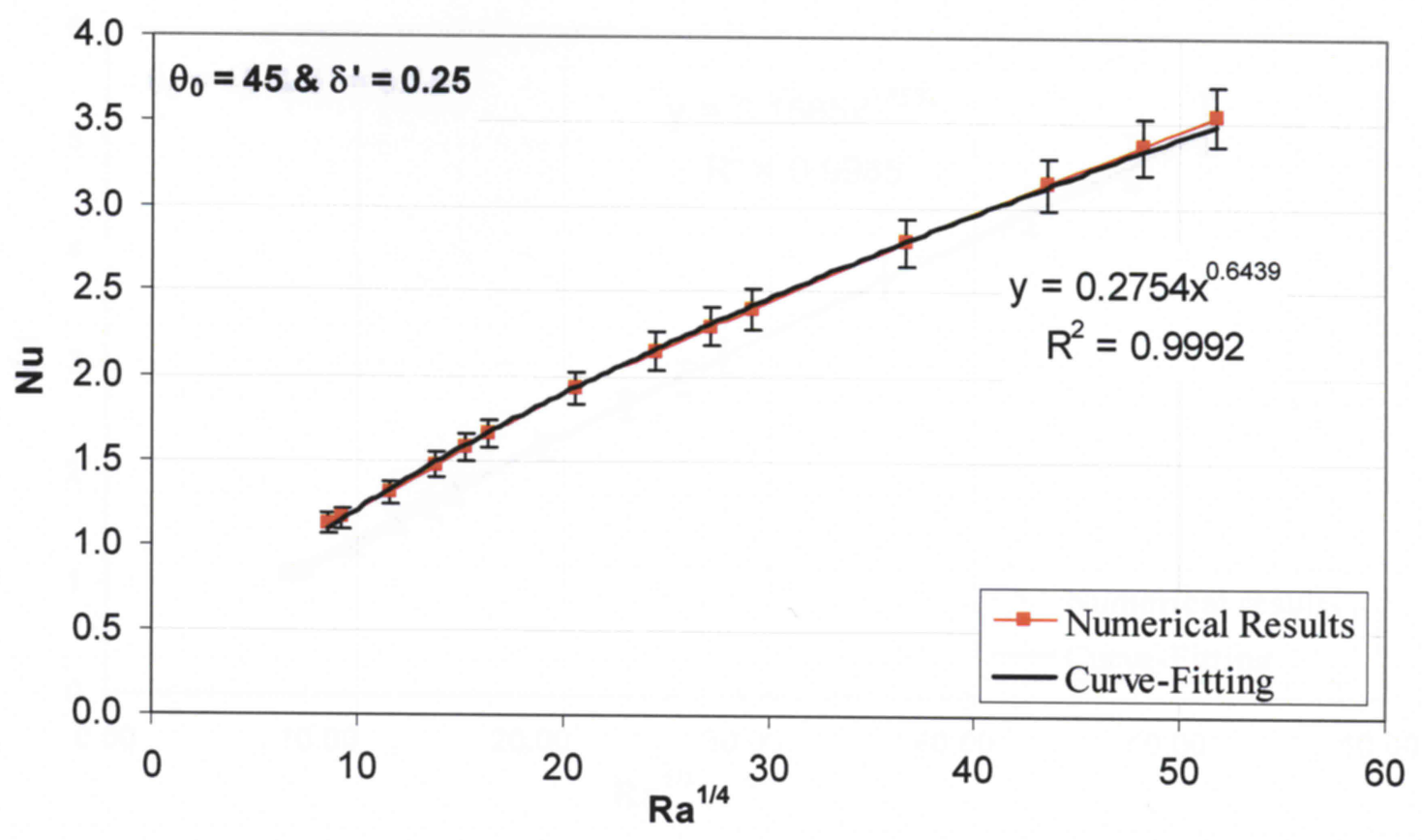

Figure B.24: Curve-fitting to the numerical results for $\theta_{0}=45^{\circ}$ and $\delta '=0.25$

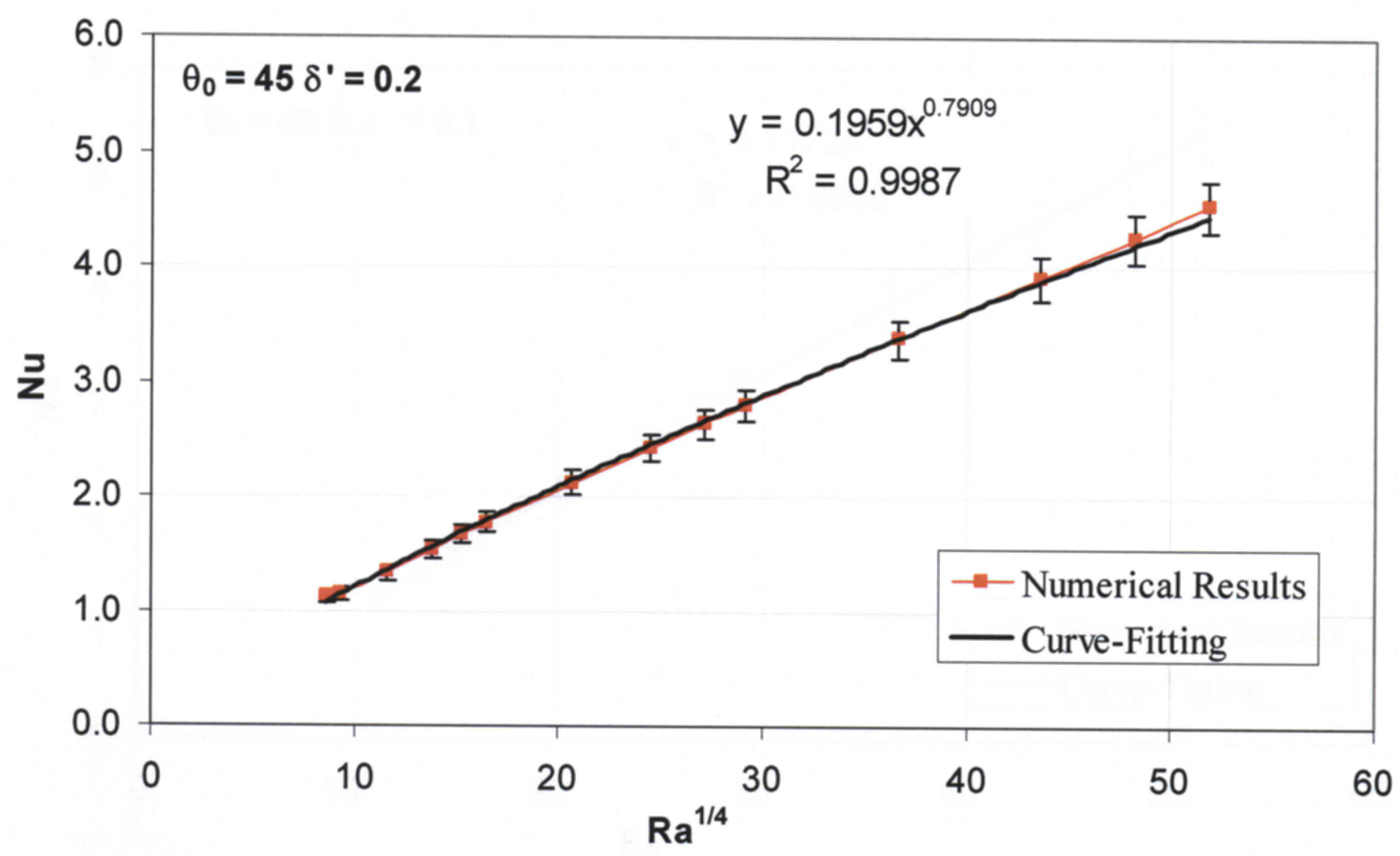

Figure B.25: Curve-fitting to the numerical results for $\theta_{0}=45^{\circ}$ and $\delta^{\prime}=0.2$ 


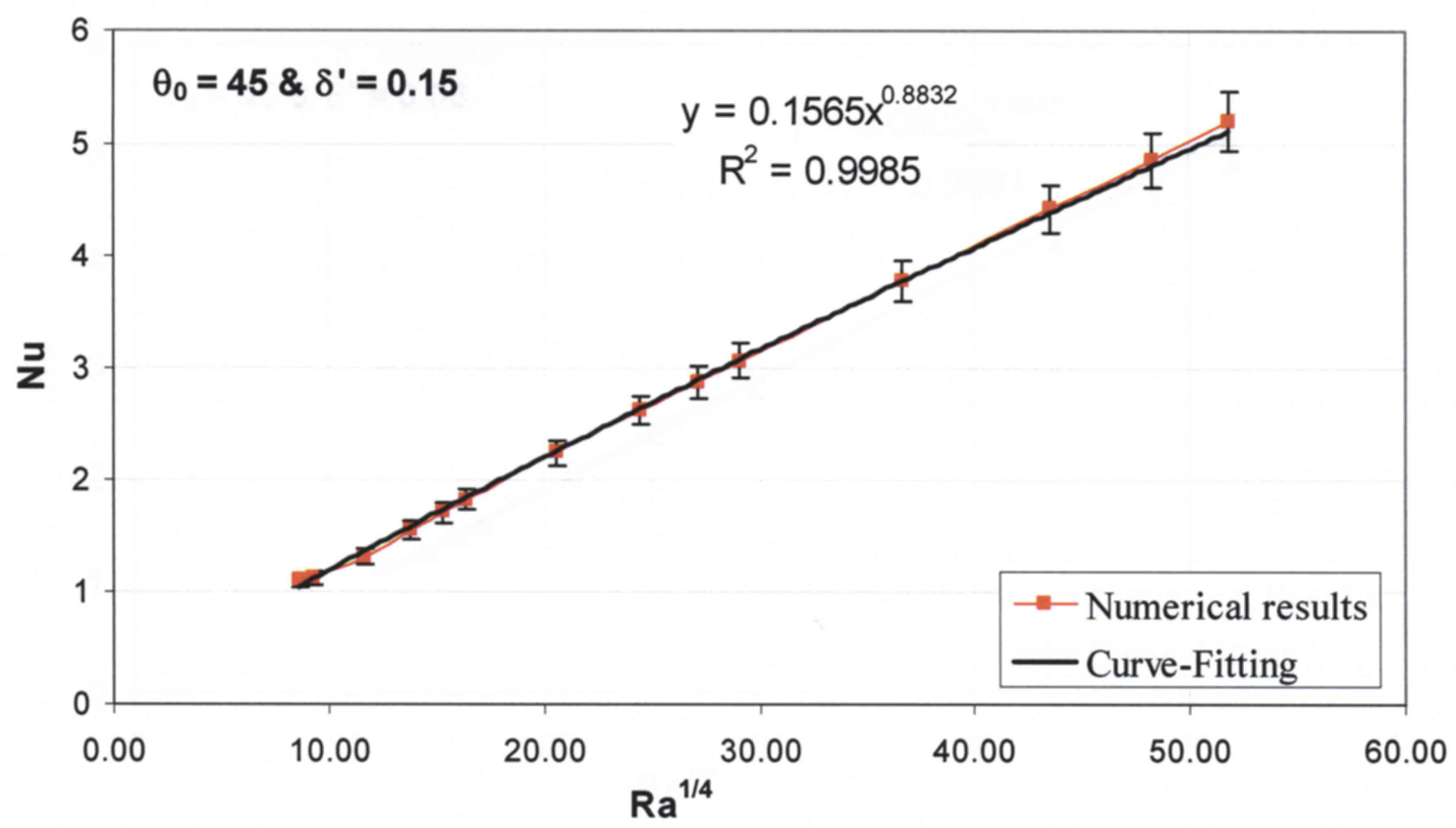

Figure B.26: Curve-fitting to the numerical results for $\theta_{0}=45^{\circ}$ and $\delta^{\prime}=0.15$

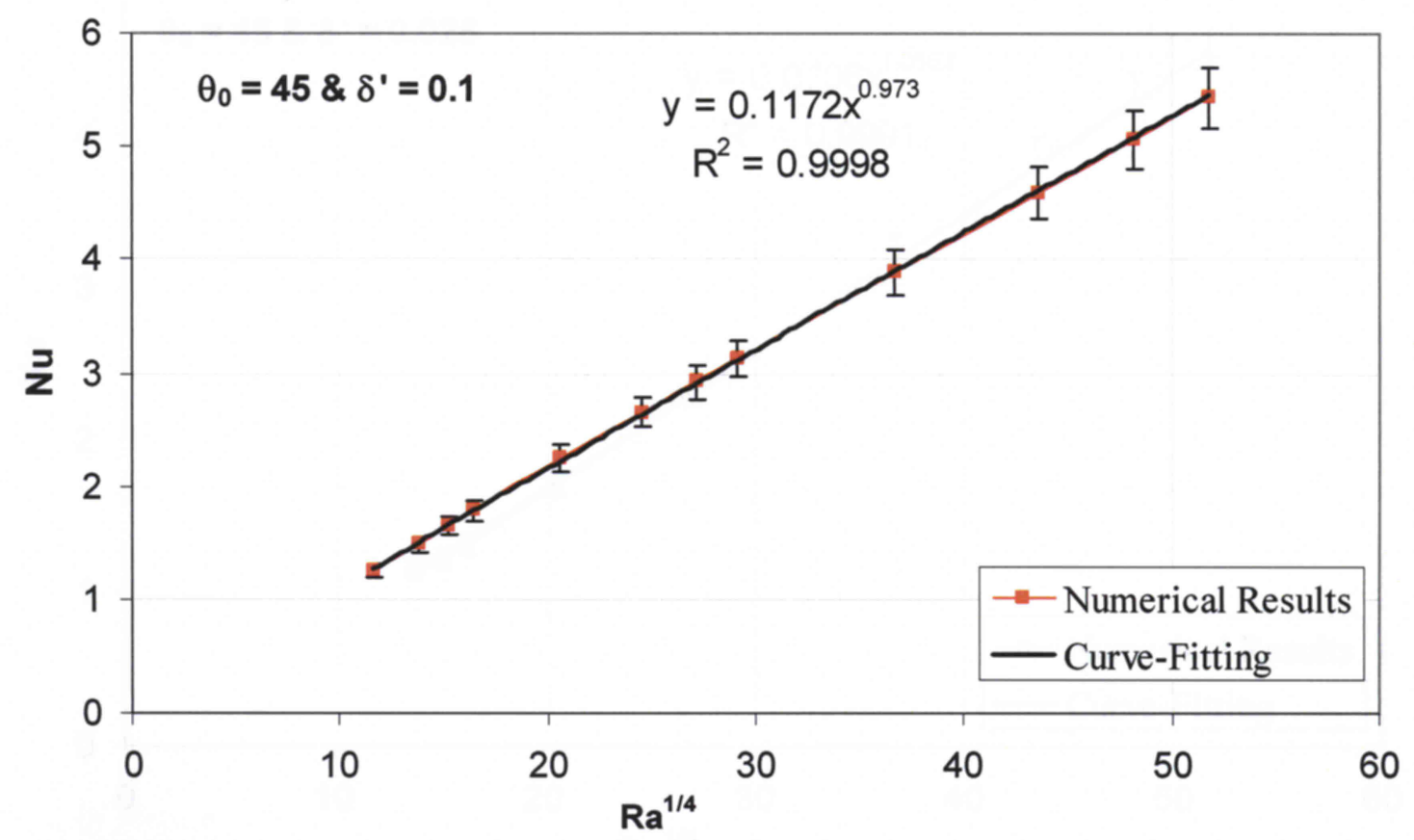

Figure B.27: Curve-fitting to the numerical results for $\theta_{0}=45^{\circ}$ and $\delta^{\prime}=0.1$ 


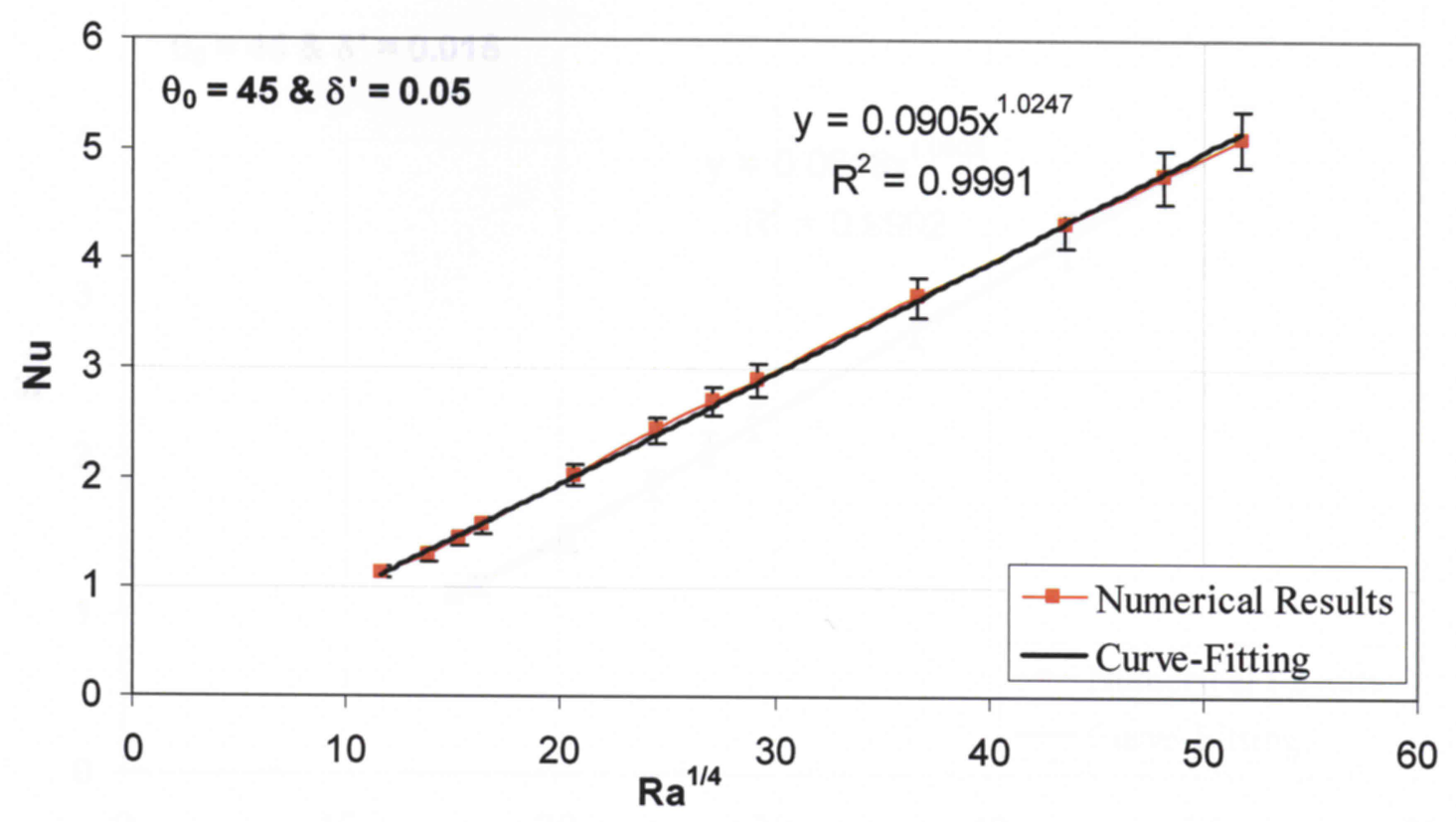

Figure B.28: Curve-fitting to the numerical results for $\theta_{0}=45^{\circ}$ and $\delta^{\prime}=0.05$

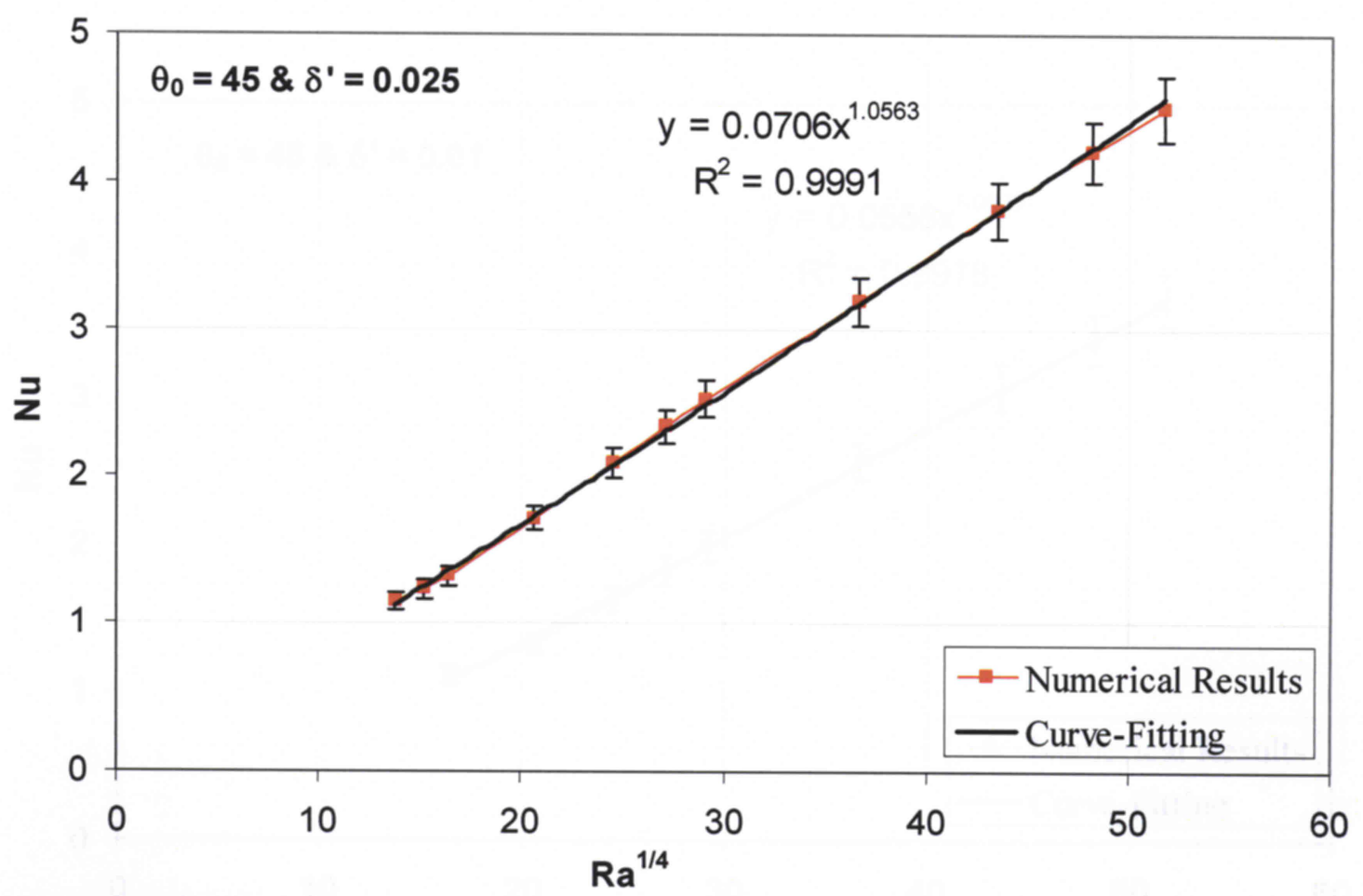

Figure B.29: Curve-fitting to the numerical results for $\theta_{0}=45^{\circ}$ and $\delta^{\prime}=0.025$ 


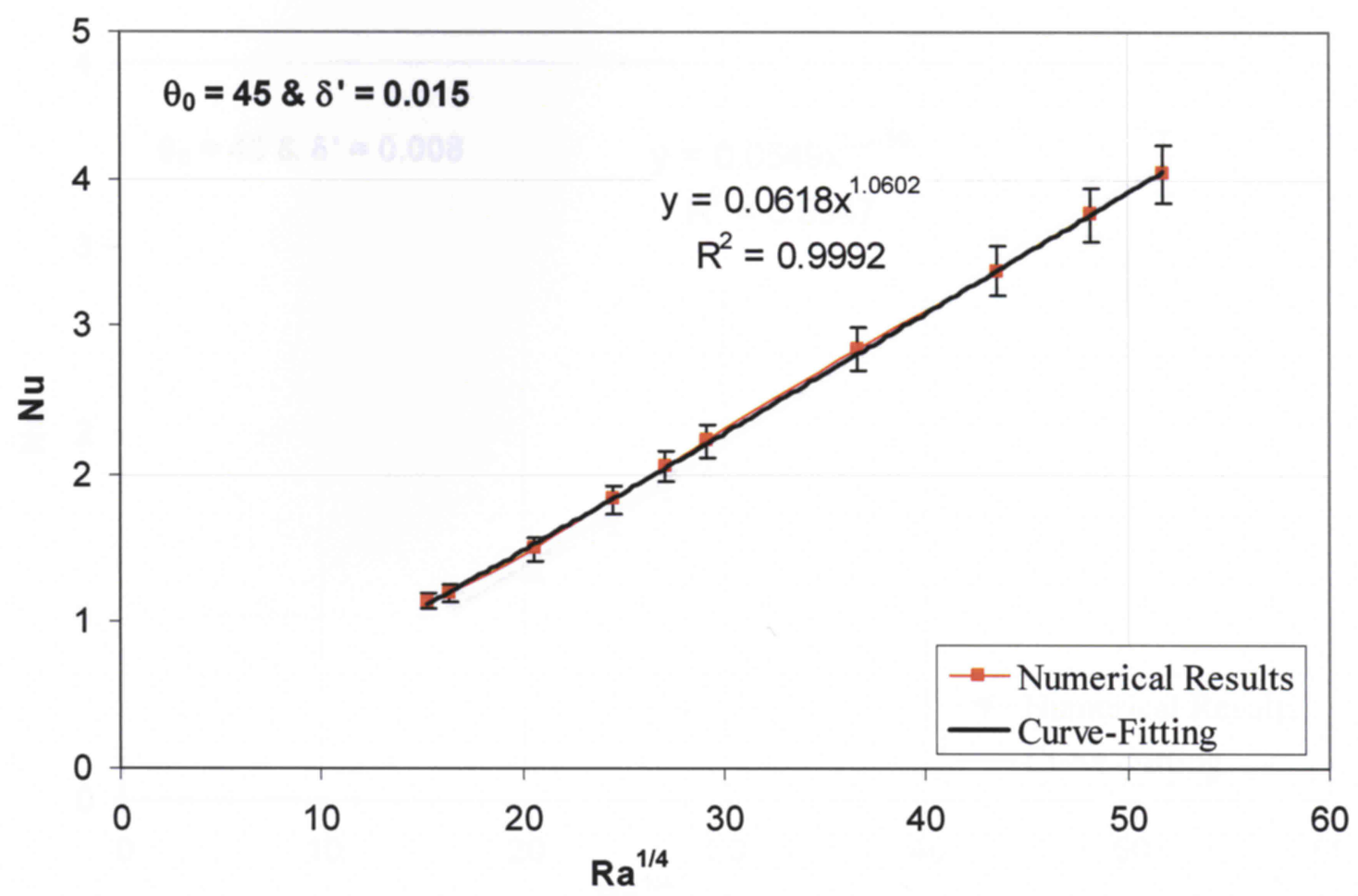

Figure B.30: Curve-fitting to the numerical results for $\theta_{0}=45^{\circ}$ and $\delta^{\prime}=0.015$

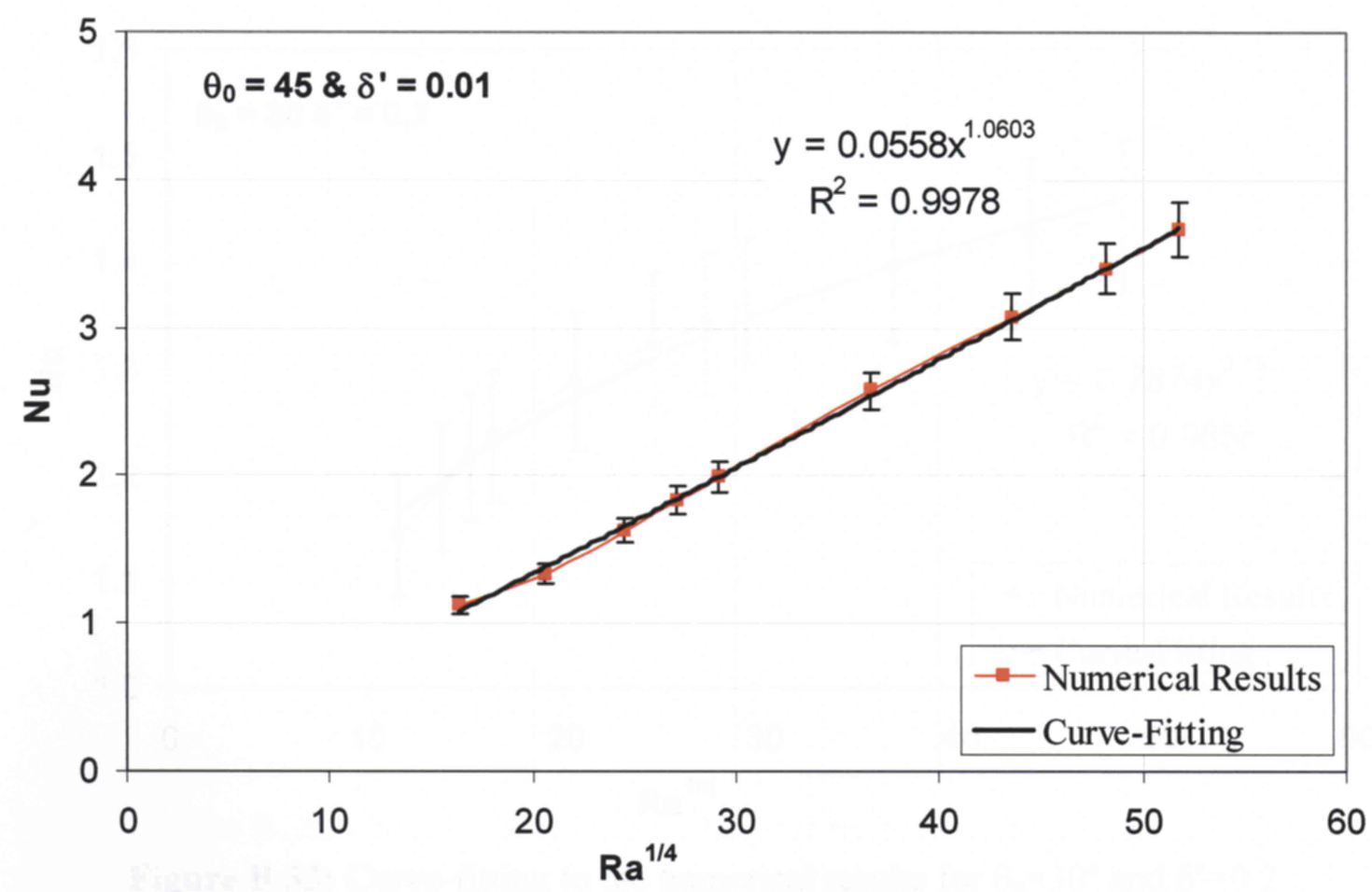

Figure B.31: Curve-fitting to the numerical results for $\theta_{0}=45^{\circ}$ and $\delta^{\prime}=0.01$ 


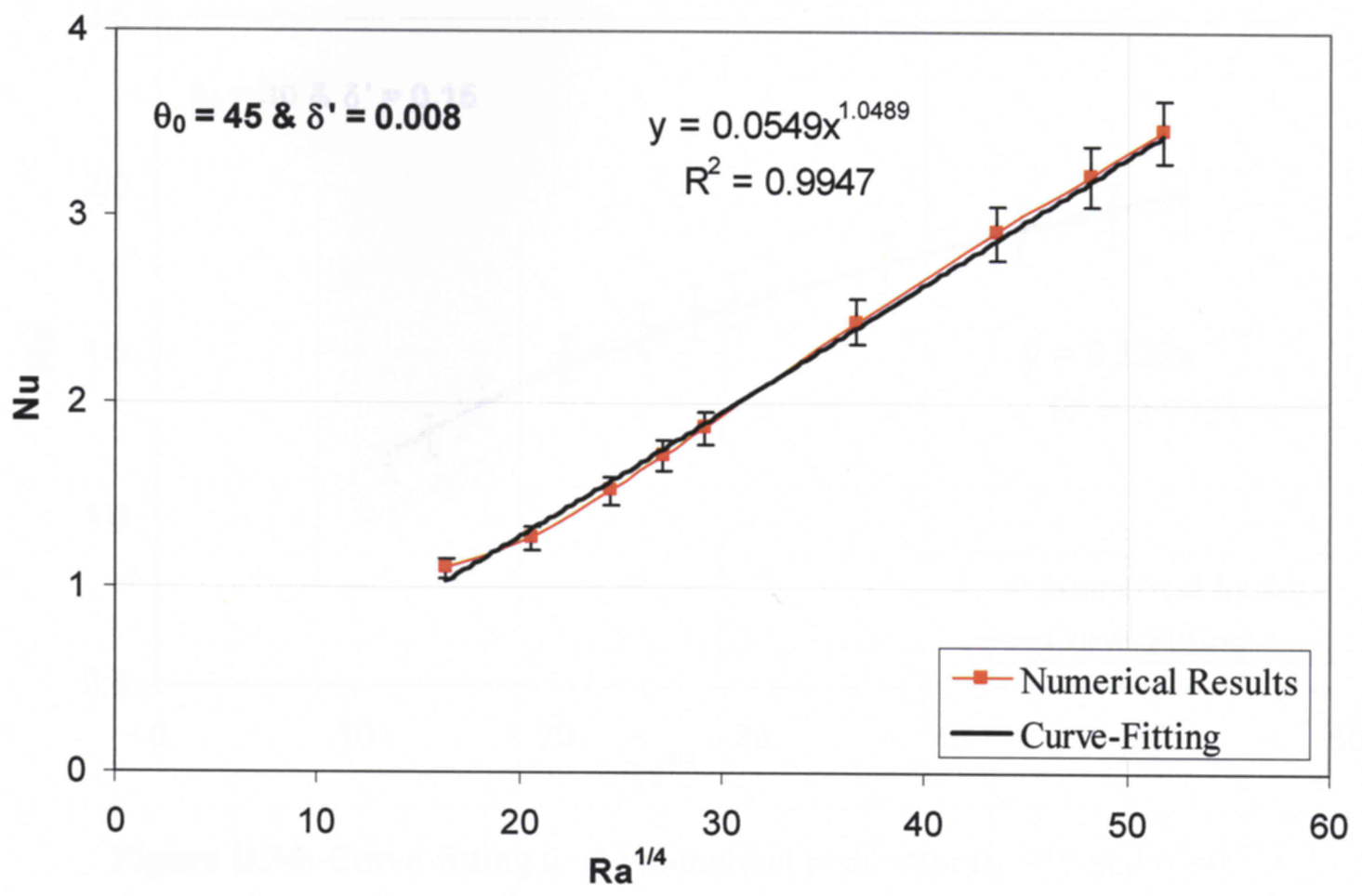

Figure B.32: Curve-fitting to the numerical results for $\theta_{0}=45^{\circ}$ and $\delta^{\prime}=0.008$

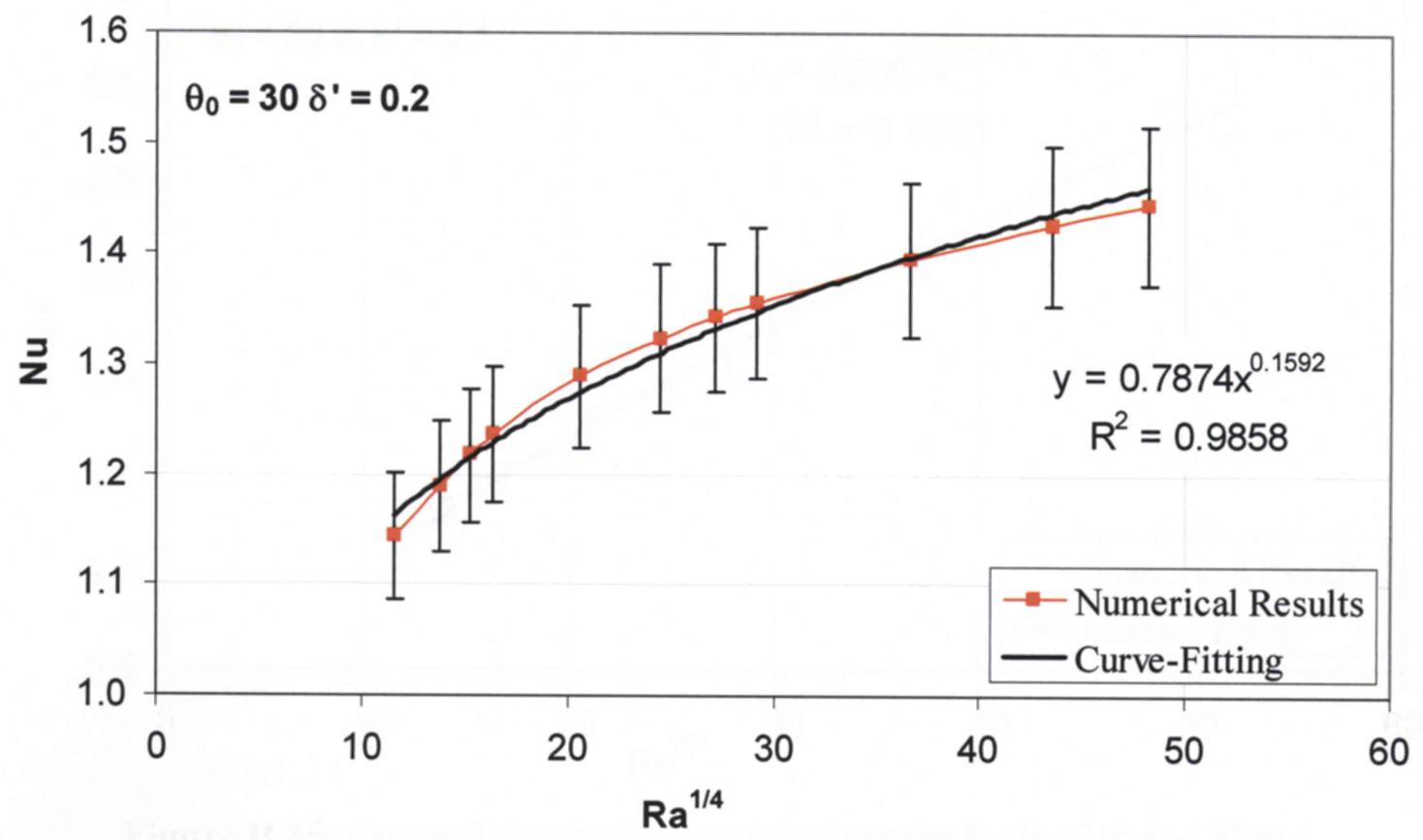

Figure B.33: Curve-fitting to the numerical results for $\theta_{0}=30^{\circ}$ and $\delta{ }^{\prime}=0.2$ 


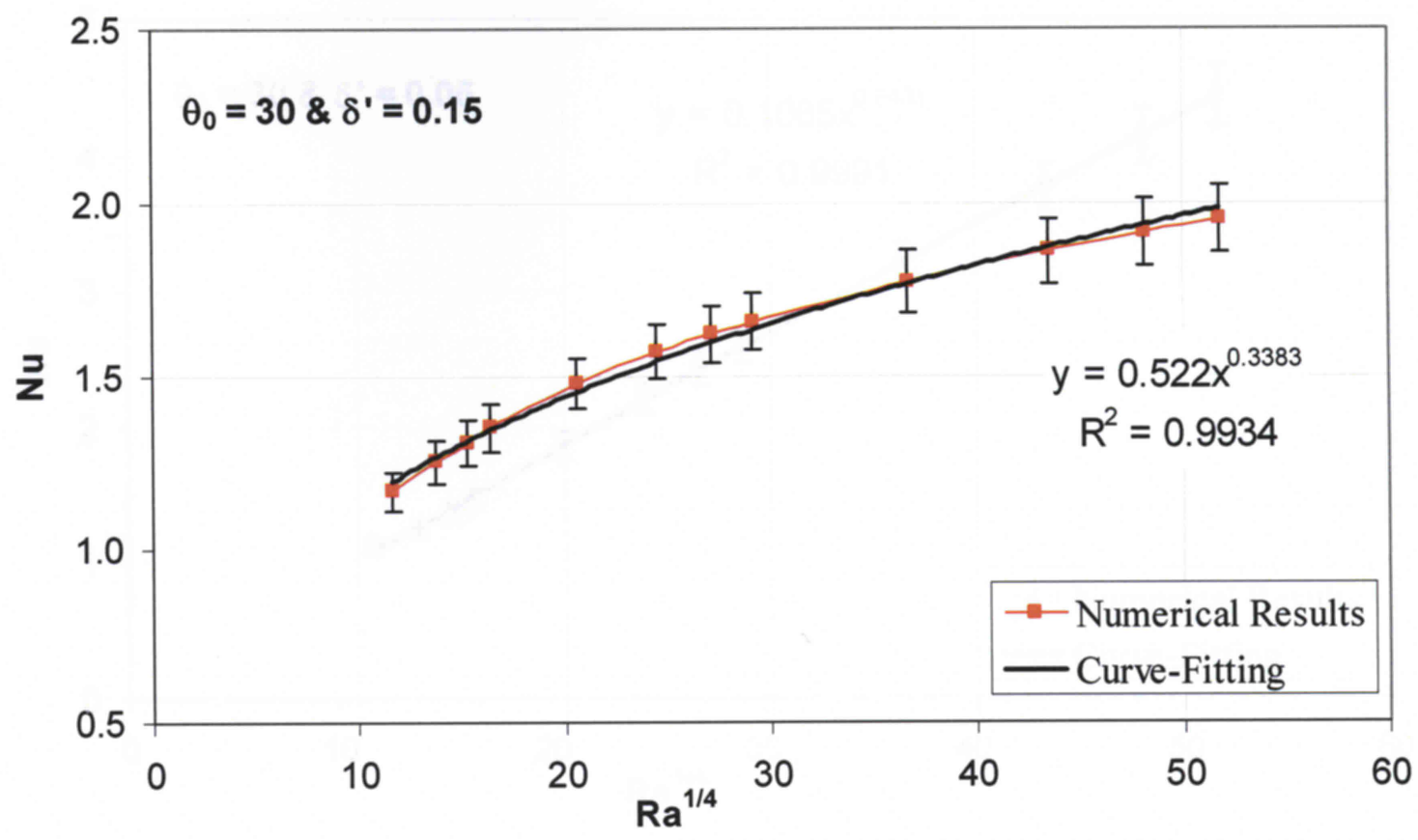

Figure B.34: Curve-fitting to the numerical results for $\theta_{0}=30^{\circ}$ and $\delta^{\prime}=0.15$



Figure B.35: Curve-fitting to the numerical results for $\theta_{0}=30^{\circ}$ and $\delta^{\prime}=0.1$ 


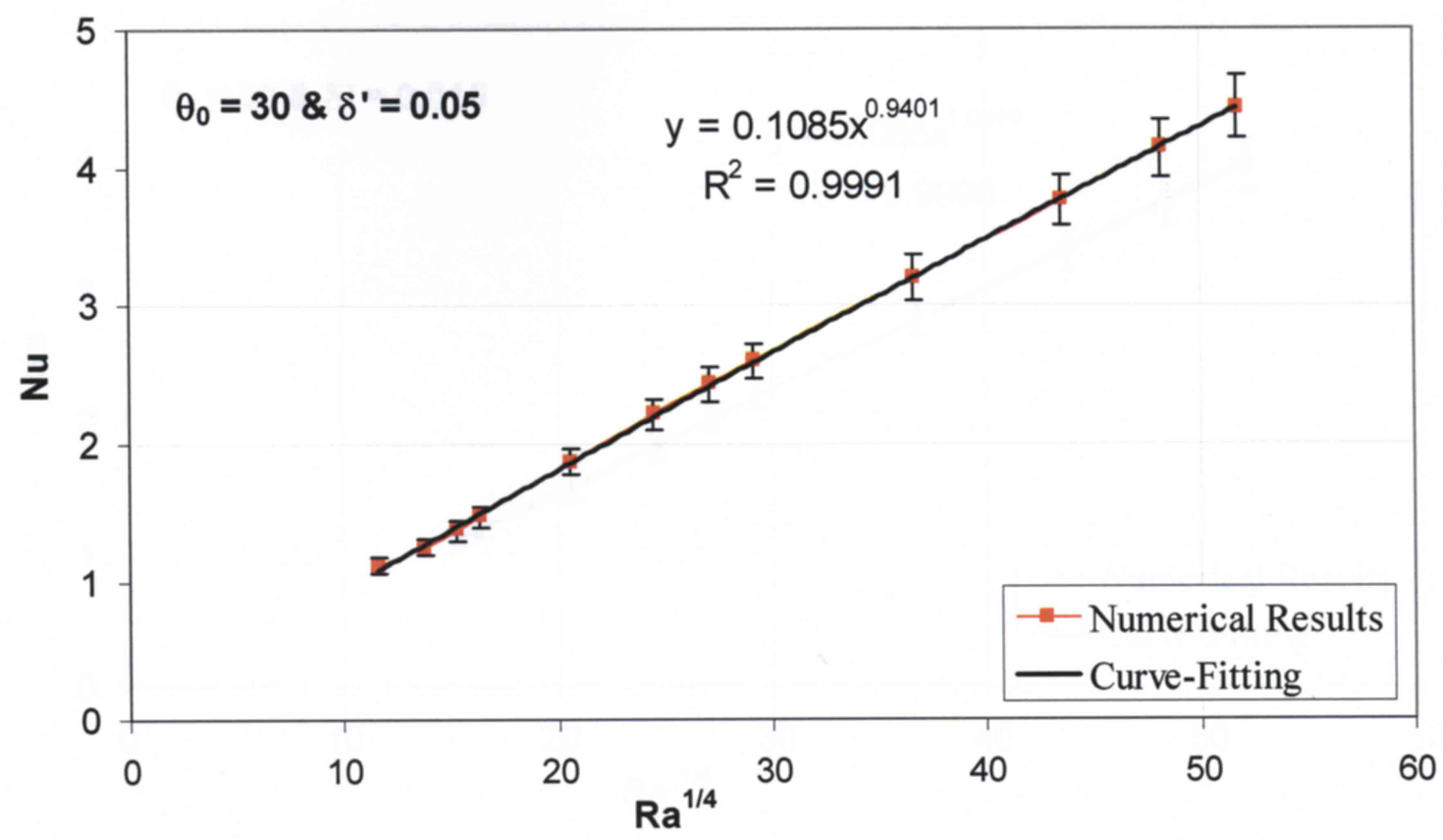

Figure B.36: Curve-fitting to the numerical results for $\theta_{0}=30^{\circ}$ and $\delta^{\prime}=0.05$

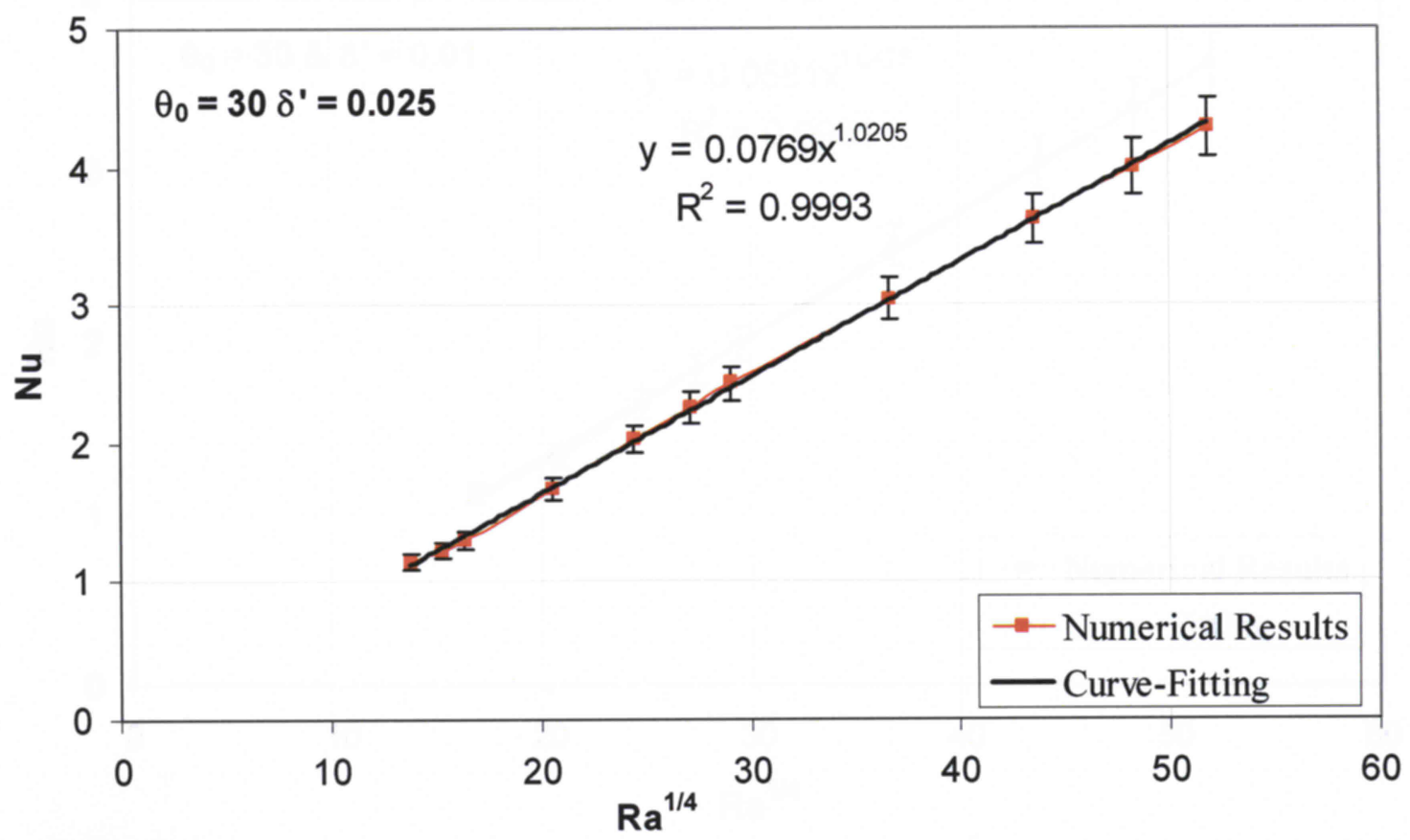

Figure B.37: Curve-fitting to the numerical results for $\theta_{0}=30^{\circ}$ and $\delta^{\prime}=0.025$ 


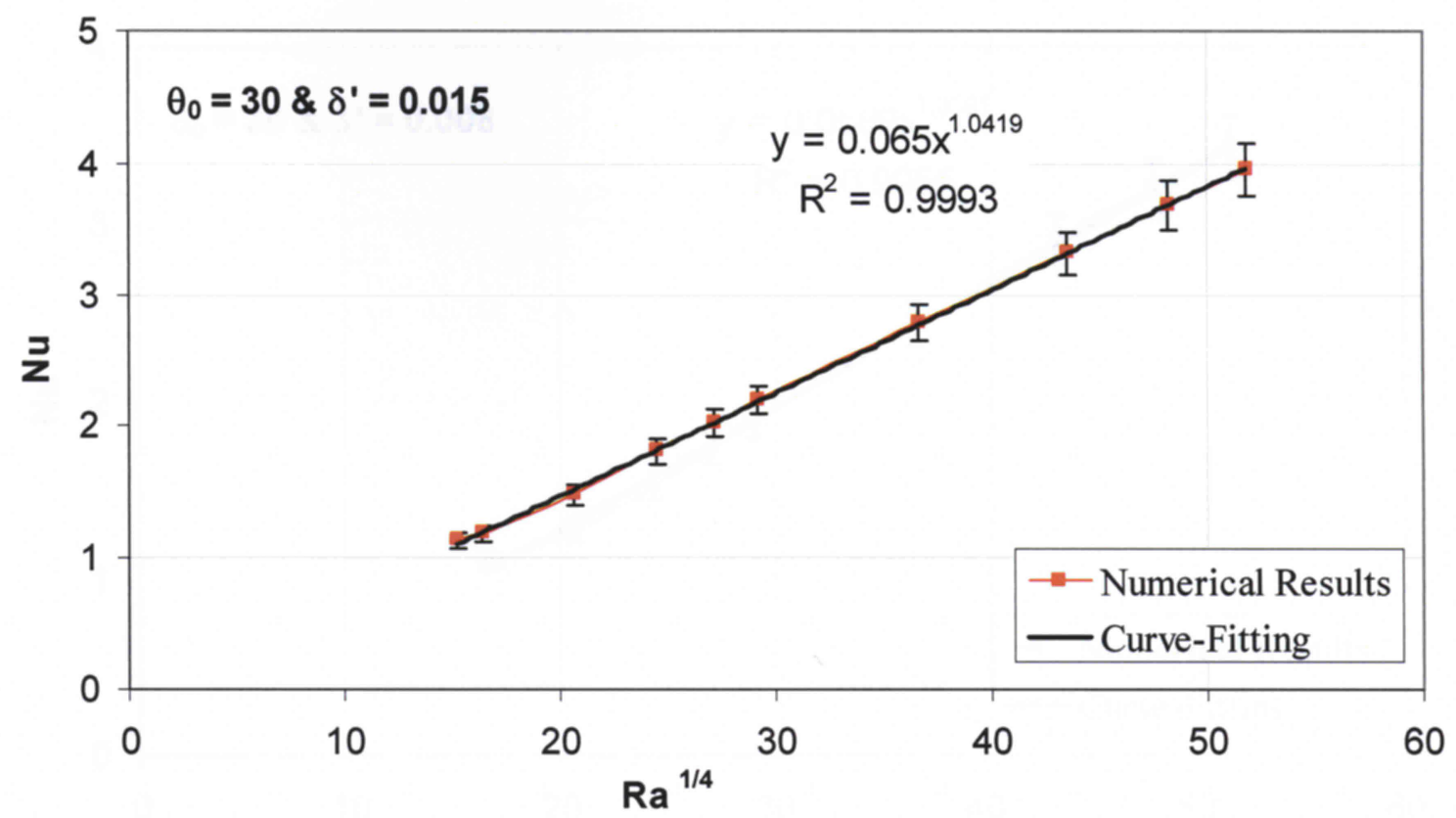

Figure B.38: Curve-fitting to the numerical results for $\theta_{0}=30^{\circ}$ and $\delta^{\prime}=0.015$

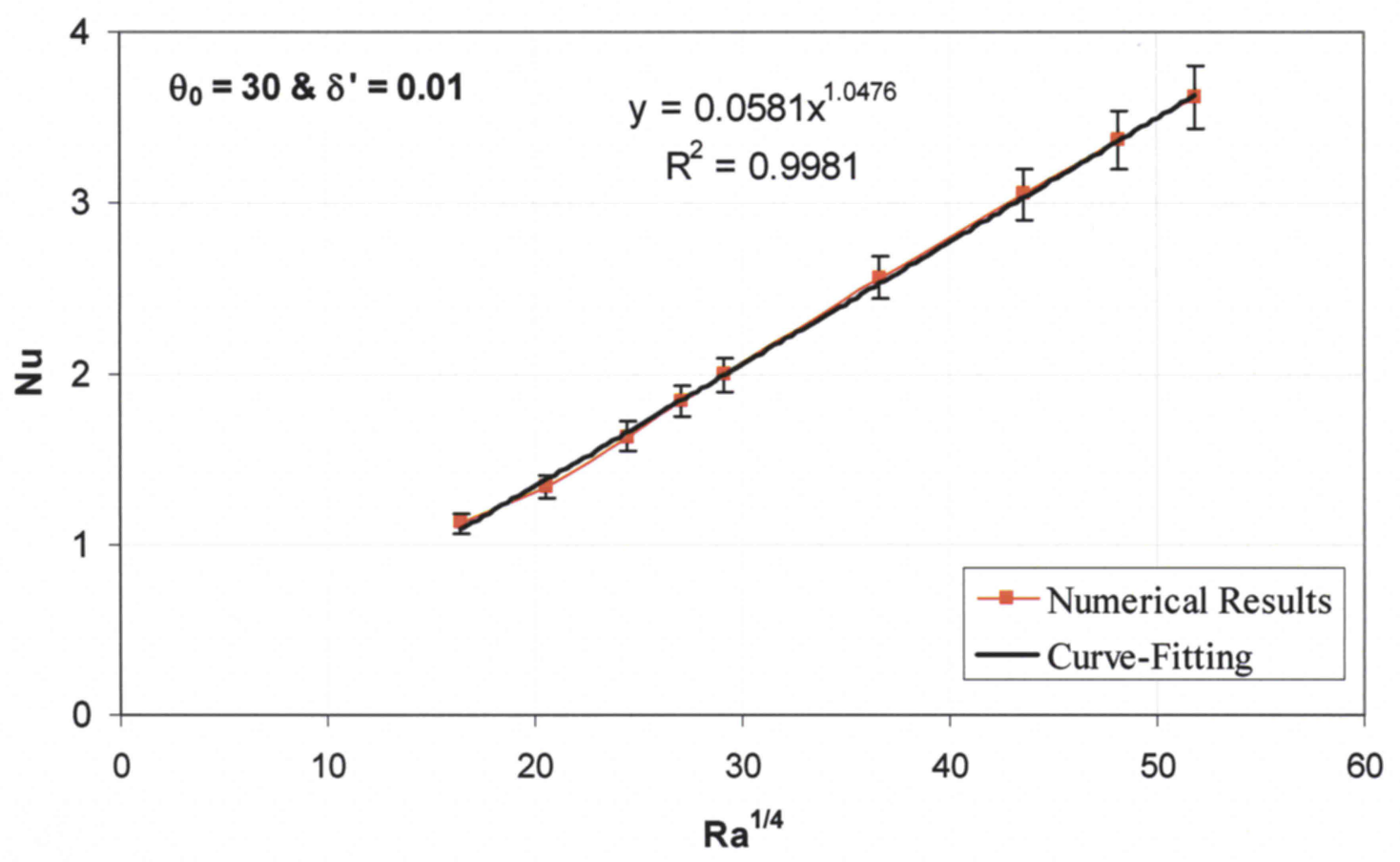

Figure B.39: Curve-fitting to the numerical results for $\theta_{0}=30^{\circ}$ and $\delta^{\prime}=0.01$ 


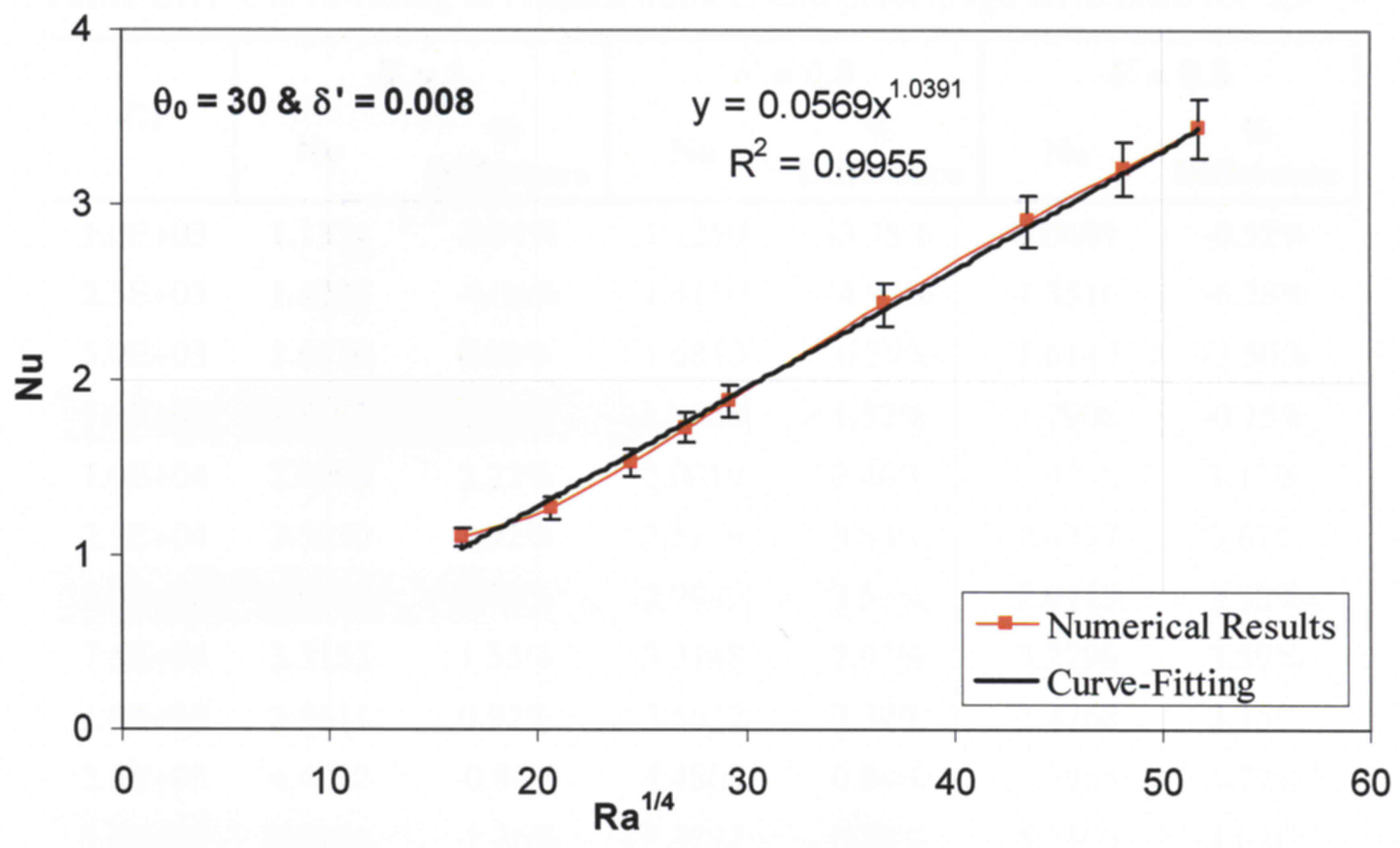

Figure B.40: Curve-fitting to the numerical results for $\theta_{0}=30^{\circ}$ and $\delta^{\prime}=0.008$ 
Table B.7: Curve-fitting to Nusselt number and percentage difference for $\theta_{0}=90^{\circ}$

\begin{tabular}{|c|c|c|c|c|c|c|}
\hline \multirow[b]{2}{*}{ Gr } & \multicolumn{2}{|c|}{$\delta^{\prime}=1$} & \multicolumn{2}{|c|}{$\delta^{\prime}=0.8$} & \multicolumn{2}{|c|}{$\delta^{\prime}=0.5$} \\
\hline & $\mathrm{Nu}$ & $\begin{array}{c}\% \\
\text { Difference }\end{array}$ & $\mathrm{Nu}$ & $\begin{array}{c}\% \\
\text { Difference } \\
\end{array}$ & $\mathrm{Nu}$ & $\begin{array}{c}\% \\
\text { Difference }\end{array}$ \\
\hline $1.0 \mathrm{E}+03$ & 1.1334 & $-3.91 \%$ & 1.1250 & $-3.78 \%$ & 1.0689 & $-0.52 \%$ \\
\hline $2.5 \mathrm{E}+03$ & 1.4233 & $-4.06 \%$ & 1.4150 & $-4.97 \%$ & 1.3516 & $-6.28 \%$ \\
\hline $5.0 \mathrm{E}+03$ & 1.6910 & $0.03 \%$ & 1.6830 & $-0.39 \%$ & 1.6142 & $-2.50 \%$ \\
\hline $7.5 \mathrm{E}+03$ & 1.8703 & $1.55 \%$ & 1.8628 & $1.52 \%$ & 1.7908 & $-0.15 \%$ \\
\hline $1.0 \mathrm{E}+04$ & 2.0090 & $2.23 \%$ & 2.0019 & $2.46 \%$ & 1.9278 & $1.17 \%$ \\
\hline $2.5 \mathrm{E}+04$ & 2.5230 & $2.92 \%$ & 2.5179 & $3.84 \%$ & 2.4377 & $3.67 \%$ \\
\hline $5.0 \mathrm{E}+04$ & 2.9974 & $2.30 \%$ & 2.9949 & $3.56 \%$ & 2.9113 & $3.98 \%$ \\
\hline $7.5 \mathrm{E}+04$ & 3.3153 & $1.55 \%$ & 3.3148 & $2.94 \%$ & 3.2298 & $3.59 \%$ \\
\hline $1.0 \mathrm{E}+05$ & 3.5611 & $0.92 \%$ & 3.5622 & $2.39 \%$ & 3.4768 & $3.15 \%$ \\
\hline $2.5 \mathrm{E}+05$ & 4.4722 & $-0.80 \%$ & 4.4805 & $0.84 \%$ & 4.3965 & $1.77 \%$ \\
\hline $5.0 \mathrm{E}+05$ & 5.3132 & $-1.36 \%$ & 5.3292 & $0.28 \%$ & 5.2505 & $1.02 \%$ \\
\hline $7.5 \mathrm{E}+05$ & 5.8767 & $-1.45 \%$ & 5.8984 & $0.13 \%$ & 5.8251 & $0.65 \%$ \\
\hline $1.0 \mathrm{E}+06$ & 6.3124 & $-1.46 \%$ & 6.3388 & $0.07 \%$ & 6.2705 & $0.39 \%$ \\
\hline $2.5 \mathrm{E}+06$ & 7.9273 & $-1.38 \%$ & 7.9727 & $0.01 \%$ & 7.9292 & $-0.16 \%$ \\
\hline $5.0 \mathrm{E}+06$ & 9.4181 & $-1.24 \%$ & 9.4831 & $0.14 \%$ & 9.4695 & $-0.36 \%$ \\
\hline $7.5 \mathrm{E}+06$ & 10.4169 & $-1.17 \%$ & 10.4960 & $0.19 \%$ & 10.5058 & $-2.03 \%$ \\
\hline $1.0 \mathrm{E}+07$ & 11.1892 & $-1.14 \%$ & 11.2796 & $0.20 \%$ & 11.3091 & $-2.20 \%$ \\
\hline $\begin{array}{l}\text { Aver } \\
\text { Diffe }\end{array}$ & $\begin{array}{l}\% \\
\text { ce: }\end{array}$ & $1.73 \%$ & & $1.63 \%$ & & $1.98 \%$ \\
\hline
\end{tabular}


Table B.7: Continued

\begin{tabular}{c|cc|cc|cc}
\hline \multirow{2}{*}{$\mathbf{G r}$} & \multicolumn{2}{|c|}{$\delta^{\prime}=\mathbf{0 . 4}$} & \multicolumn{2}{c|}{$\delta^{\prime}=\mathbf{0 . 3}$} & \multicolumn{2}{c}{$\delta^{\prime}=\mathbf{0 . 2}$} \\
\cline { 2 - 7 } & $\mathbf{N u}$ & $\begin{array}{c}\% \\
\text { Difference }\end{array}$ & $\mathbf{N u}$ & $\begin{array}{c}\% \\
\text { Difference }\end{array}$ & Nu & $\begin{array}{c}\% \\
\text { Difference }\end{array}$ \\
\hline $1.0 \mathrm{E}+03$ & 1.0284 & $2.36 \%$ & 1.0000 & $3.99 \%$ & 1.0000 & $2.74 \%$ \\
$2.5 \mathrm{E}+03$ & 1.3044 & $-5.98 \%$ & 1.2376 & $-4.77 \%$ & 1.2321 & $-9.74 \%$ \\
$5.0 \mathrm{E}+03$ & 1.5613 & $-3.49 \%$ & 1.4836 & $-4.44 \%$ & 1.4744 & $-2.93 \%$ \\
\hline $7.5 \mathrm{E}+03$ & 1.7345 & $-1.23 \%$ & 1.6496 & $-2.51 \%$ & 1.6376 & $3.08 \%$ \\
\hline $1.0 \mathrm{E}+04$ & 1.8688 & $0.15 \%$ & 1.7786 & $-1.15 \%$ & 1.7643 & $5.98 \%$ \\
$2.5 \mathrm{E}+04$ & 2.3703 & $2.99 \%$ & 2.2603 & $2.03 \%$ & 2.2368 & $9.52 \%$ \\
\hline $5.0 \mathrm{E}+04$ & 2.8371 & $3.55 \%$ & 2.7096 & $2.97 \%$ & 2.6766 & $-4.90 \%$ \\
\hline $7.5 \mathrm{E}+04$ & 3.1518 & $3.21 \%$ & 3.0128 & $2.88 \%$ & 2.9729 & $-4.45 \%$ \\
$1.0 \mathrm{E}+05$ & 3.3960 & $2.79 \%$ & 3.2483 & $2.52 \%$ & 3.2029 & $-4.50 \%$ \\
$2.5 \mathrm{E}+05$ & 4.3071 & $1.36 \%$ & 4.1281 & $1.01 \%$ & 4.0606 & $-5.74 \%$ \\
\hline $5.0 \mathrm{E}+05$ & 5.1555 & $0.46 \%$ & 4.9487 & $0.09 \%$ & 4.8590 & $-6.62 \%$ \\
\hline $7.5 \mathrm{E}+05$ & 5.7273 & $-0.01 \%$ & 5.5025 & $-0.35 \%$ & 5.3970 & $6.21 \%$ \\
$1.0 \mathrm{E}+06$ & 6.1710 & $-0.33 \%$ & 5.9325 & $-0.89 \%$ & 5.8144 & $5.57 \%$ \\
$2.5 \mathrm{E}+06$ & 7.8267 & $-1.23 \%$ & 7.5394 & $0.15 \%$ & 7.3715 & $-3.08 \%$ \\
\hline $5.0 \mathrm{E}+06$ & 9.3684 & $-2.99 \%$ & 9.0382 & $-1.14 \%$ & 8.8209 & $1.32 \%$ \\
\hline $7.5 \mathrm{E}+06$ & 10.4074 & $-3.37 \%$ & 10.0495 & $-1.78 \%$ & 9.7975 & $0.71 \%$ \\
$1.0 \mathrm{E}+07$ & 11.2137 & $-3.65 \%$ & 10.8349 & $-2.34 \%$ & 10.5553 & $-0.38 \%$ \\
\hline Average \% & $2.30 \%$ & & $2.06 \%$ & & $4.56 \%$ \\
\hline Difference: & & & & & & \\
\hline
\end{tabular}


Table B.7: Continued

\begin{tabular}{|c|c|c|c|c|}
\hline \multirow[b]{2}{*}{$\mathbf{G r}$} & \multicolumn{2}{|c|}{$\delta^{\prime}=0.1$} & \multicolumn{2}{|c|}{$\delta^{\prime}=0.01$} \\
\hline & $\mathrm{Nu}$ & $\begin{array}{c}\% \\
\text { Difference }\end{array}$ & $\mathrm{Nu}$ & $\begin{array}{c}\% \\
\text { Difference }\end{array}$ \\
\hline $1.0 \mathrm{E}+03$ & 1.0000 & $1.38 \%$ & 1.0000 & $0.08 \%$ \\
\hline $2.5 \mathrm{E}+03$ & 1.1514 & $-8.55 \%$ & 1.0000 & $0.42 \%$ \\
\hline $5.0 \mathrm{E}+03$ & 1.3714 & $-11.26 \%$ & 1.0000 & $1.17 \%$ \\
\hline $7.5 E+03$ & 1.5192 & $-3.55 \%$ & 1.0000 & $1.96 \%$ \\
\hline $1.0 \mathrm{E}+04$ & 1.6336 & $2.91 \%$ & 1.0000 & $2.74 \%$ \\
\hline $2.5 \mathrm{E}+04$ & 2.0585 & $10.58 \%$ & 1.0866 & $0.37 \%$ \\
\hline $5.0 \mathrm{E}+04$ & 2.4520 & $8.45 \%$ & 1.2893 & $0.75 \%$ \\
\hline $7.5 \mathrm{E}+04$ & 2.7161 & $1.63 \%$ & 1.4249 & $2.09 \%$ \\
\hline $1.0 \mathrm{E}+05$ & 2.9207 & $0.89 \%$ & 1.5297 & $1.13 \%$ \\
\hline $2.5 \mathrm{E}+05$ & 3.6804 & $2.74 \%$ & 1.9178 & $-2.90 \%$ \\
\hline $5.0 \mathrm{E}+05$ & 4.3839 & $2.16 \%$ & 2.2755 & $-2.09 \%$ \\
\hline $7.5 \mathrm{E}+05$ & 4.8562 & $2.02 \%$ & 2.5149 & $-1.72 \%$ \\
\hline $1.0 \mathrm{E}+06$ & 5.2218 & $3.68 \%$ & 2.6999 & $-1.22 \%$ \\
\hline $2.5 \mathrm{E}+06$ & 6.5802 & $-1.72 \%$ & 3.3847 & $0.51 \%$ \\
\hline $5.0 \mathrm{E}+06$ & 7.8379 & $-3.64 \%$ & 4.0160 & $1.68 \%$ \\
\hline $7.5 \mathrm{E}+06$ & 8.6823 & $-4.61 \%$ & 4.4386 & $0.70 \%$ \\
\hline $1.0 \mathrm{E}+07$ & 9.3360 & $-4.80 \%$ & 4.7651 & $0.52 \%$ \\
\hline $\begin{array}{l}\text { Ave } \\
\text { Diff }\end{array}$ & & $4.39 \%$ & & $1.30 \%$ \\
\hline
\end{tabular}


Table B.8: Curve-fitting to Nusselt number and percentage difference for $\theta_{0}=45^{\circ}$

\begin{tabular}{c|cc|cc|cc}
\hline \multirow{2}{*}{$\mathbf{G r}$} & \multicolumn{2}{|c|}{$\delta^{\prime}=\mathbf{1}$} & \multicolumn{2}{c|}{$\delta^{\prime}=\mathbf{0 . 8}$} & \multicolumn{2}{c}{$\delta^{\prime}=\mathbf{0 . 5}$} \\
\cline { 2 - 7 } & $\mathbf{N u}$ & $\begin{array}{c}\% \\
\text { Difference }\end{array}$ & $\mathbf{N u}$ & $\begin{array}{c}\text { \% } \\
\text { Difference }\end{array}$ & Nu & $\begin{array}{c}\% \\
\text { Difference }\end{array}$ \\
\hline $1.0 \mathrm{E}+03$ & 1.0220 & $0.81 \%$ & 1.1074 & $-6.09 \%$ & 1.1584 & $-10.03 \%$ \\
$2.5 \mathrm{E}+03$ & 1.2939 & $-4.14 \%$ & 1.4020 & $-7.23 \%$ & 1.4666 & $-9.41 \%$ \\
$5.0 \mathrm{E}+03$ & 1.5468 & $1.50 \%$ & 1.6760 & $-0.36 \%$ & 1.7532 & $-0.78 \%$ \\
$7.5 \mathrm{E}+03$ & 1.7170 & $3.72 \%$ & 1.8604 & $1.98 \%$ & 1.9461 & $2.13 \%$ \\
$1.0 \mathrm{E}+04$ & 1.8490 & $4.59 \%$ & 2.0035 & $2.87 \%$ & 2.0958 & $3.35 \%$ \\
$2.5 \mathrm{E}+04$ & 2.3410 & $5.00 \%$ & 2.5366 & $3.23 \%$ & 2.6535 & $4.50 \%$ \\
$5.0 \mathrm{E}+04$ & 2.7985 & $4.57 \%$ & 3.0323 & $2.37 \%$ & 3.1720 & $3.92 \%$ \\
$7.5 \mathrm{E}+04$ & 3.1065 & $3.83 \%$ & 3.3660 & $1.70 \%$ & 3.5211 & $3.28 \%$ \\
$1.0 \mathrm{E}+05$ & 3.3453 & $3.50 \%$ & 3.6248 & $1.22 \%$ & 3.7918 & $2.73 \%$ \\
$2.5 \mathrm{E}+05$ & 4.2355 & $2.72 \%$ & 4.5894 & $-0.15 \%$ & 4.8008 & $0.81 \%$ \\
$5.0 \mathrm{E}+05$ & 5.0632 & $2.56 \%$ & 5.4862 & $-0.80 \%$ & 5.7389 & $-0.39 \%$ \\
$7.5 \mathrm{E}+05$ & 5.6204 & $2.62 \%$ & 6.0899 & $-1.02 \%$ & 6.3705 & $-0.95 \%$ \\
$1.0 \mathrm{E}+06$ & 6.0526 & $2.71 \%$ & 6.5582 & $-1.10 \%$ & 6.8603 & $-1.25 \%$ \\
$2.5 \mathrm{E}+06$ & 7.6632 & $3.48 \%$ & 8.3034 & $-0.56 \%$ & 8.6859 & $-1.48 \%$ \\
$5.0 \mathrm{E}+06$ & 9.1606 & $4.04 \%$ & 9.9259 & $-0.31 \%$ & 10.3832 & $-1.47 \%$ \\
$7.5 \mathrm{E}+06$ & 10.1688 & $4.20 \%$ & 11.0183 & $-0.30 \%$ & 11.5259 & $-1.52 \%$ \\
$1.0 \mathrm{E}+07$ & 10.9506 & $4.25 \%$ & 11.8655 & $-0.35 \%$ & 12.4121 & $-1.61 \%$ \\
Average \% & $3.43 \%$ & & $1.86 \%$ & & $2.92 \%$ \\
$\mathrm{D}$ ifference: & & & & & \\
\hline
\end{tabular}


Table B.8: Continued

\begin{tabular}{|c|c|c|c|c|c|c|}
\hline \multirow[b]{2}{*}{$\mathbf{G r}$} & \multicolumn{2}{|c|}{$\delta^{\prime}=0.4$} & \multicolumn{2}{|c|}{$\delta^{\prime}=0.3$} & \multicolumn{2}{|c|}{$\delta^{\prime}=0.2$} \\
\hline & $\mathbf{N u}$ & $\begin{array}{c}\% \% \\
\text { Difference } \\
\end{array}$ & $\mathbf{N u}$ & $\begin{array}{c}\% \\
\text { Difference } \\
\end{array}$ & $\mathrm{Nu}$ & $\begin{array}{c}\% \\
\text { Difference }\end{array}$ \\
\hline $1.0 \mathrm{E}+03$ & 1.1294 & $-7.62 \%$ & 1.1835 & $-13.55 \%$ & 2.4962 & $-141.73 \%$ \\
\hline $2.5 \mathrm{E}+03$ & 1.4299 & $-9.10 \%$ & 1.4985 & $-18.90 \%$ & 3.1186 & $-26.13 \%$ \\
\hline $5.0 \mathrm{E}+03$ & 1.7094 & $-0.42 \%$ & 1.7913 & $-2.01 \%$ & 3.6906 & $-2.89 \%$ \\
\hline $7.5 \mathrm{E}+03$ & 1.8975 & $2.93 \%$ & 1.9884 & $2.85 \%$ & 4.0727 & $4.35 \%$ \\
\hline $1.0 \mathrm{E}+04$ & 2.0434 & $4.42 \%$ & 2.1413 & $4.74 \%$ & 4.3675 & $6.95 \%$ \\
\hline $2.5 \mathrm{E}+04$ & 2.5871 & $6.20 \%$ & 2.7111 & $6.92 \%$ & 5.4565 & $10.33 \%$ \\
\hline $5.0 \mathrm{E}+04$ & 3.0927 & $5.90 \%$ & 3.2409 & $6.84 \%$ & 6.4573 & $10.17 \%$ \\
\hline $7.5 \mathrm{E}+04$ & 3.4330 & $5.35 \%$ & 3.5975 & $6.34 \%$ & 7.1258 & $6.05 \%$ \\
\hline $1.0 \mathrm{E}+05$ & 3.6970 & $4.85 \%$ & 3.8741 & $5.79 \%$ & 7.6416 & $5.70 \%$ \\
\hline $2.5 \mathrm{E}+05$ & 4.6808 & $3.11 \%$ & 4.9051 & $2.77 \%$ & 9.5470 & $4.58 \%$ \\
\hline $5.0 \mathrm{E}+05$ & 5.5955 & $1.96 \%$ & 5.8636 & $-4.56 \%$ & 11.2980 & $4.05 \%$ \\
\hline $7.5 \mathrm{E}+05$ & 6.2112 & $1.40 \%$ & 6.5089 & $-5.00 \%$ & 12.4676 & $3.49 \%$ \\
\hline $1.0 \mathrm{E}+06$ & 6.6888 & $1.27 \%$ & 7.0093 & $-5.16 \%$ & 13.3702 & $3.47 \%$ \\
\hline $2.5 \mathrm{E}+06$ & 8.4688 & $1.09 \%$ & 8.8745 & $-5.57 \%$ & 16.7038 & $0.55 \%$ \\
\hline $5.0 \mathrm{E}+06$ & 10.1236 & $0.80 \%$ & 10.6087 & $-6.31 \%$ & 19.7675 & $-2.03 \%$ \\
\hline $7.5 \mathrm{E}+06$ & 11.2377 & $0.51 \%$ & 11.7762 & $-6.21 \%$ & 21.8139 & $-2.29 \%$ \\
\hline $1.0 \mathrm{E}+07$ & 12.1018 & $0.23 \%$ & 12.6817 & $-6.37 \%$ & 23.3931 & $-2.34 \%$ \\
\hline $\begin{array}{l}\text { Aver: } \\
\text { Diffe }\end{array}$ & $\begin{array}{l}\text { ge } \% \\
\text { ence: }\end{array}$ & $3.36 \%$ & & $6.46 \%$ & & $5.96 \%$ \\
\hline
\end{tabular}


Table B.8: Continued

\begin{tabular}{|c|c|c|c|c|c|}
\hline \multirow{2}{*}{$\mathbf{G r}$} & \multicolumn{2}{|c|}{$\delta^{\prime}=0.1$} & \multirow[b]{2}{*}{$\mathbf{G r}$} & \multicolumn{2}{|c|}{$\delta^{\prime}=0.01$} \\
\hline & $\mathbf{N u}$ & $\begin{array}{c}\% \% \\
\text { Difference } \\
\end{array}$ & & $\mathrm{Nu}$ & $\begin{array}{c}\% \\
\text { Difference } \\
\end{array}$ \\
\hline $1.0 \mathrm{E}+03$ & 1.2368 & $-21.46 \%$ & $1.0 \mathrm{E}+03$ & 1.0000 & $0.09 \%$ \\
\hline $2.5 \mathrm{E}+03$ & 1.5452 & $-40.62 \%$ & $2.5 \mathrm{E}+03$ & 1.0000 & $0.51 \%$ \\
\hline $5.0 \mathrm{E}+03$ & 1.8287 & $-16.78 \%$ & $5.0 \mathrm{E}+03$ & 1.0000 & $1.69 \%$ \\
\hline $7.5 \mathrm{E}+03$ & 2.0180 & $-0.37 \%$ & $7.5 \mathrm{E}+03$ & 1.0000 & $3.04 \%$ \\
\hline $1.0 \mathrm{E}+04$ & 2.1640 & $3.17 \%$ & $8.5 \mathrm{E}+03$ & 1.0000 & $3.57 \%$ \\
\hline $2.5 \mathrm{E}+04$ & 2.7036 & $7.62 \%$ & $1.0 \mathrm{E}+04$ & 1.0000 & $4.36 \%$ \\
\hline $5.0 \mathrm{E}+04$ & 3.1995 & $8.65 \%$ & $2.0 \mathrm{E}+04$ & 1.0587 & $3.84 \%$ \\
\hline $7.5 \mathrm{E}+04$ & 3.5307 & $8.88 \%$ & $3.0 \mathrm{E}+04$ & 1.1683 & $0.04 \%$ \\
\hline $1.0 \mathrm{E}+05$ & 3.7863 & $8.77 \%$ & $4.0 \mathrm{E}+04$ & 1.2529 & $0.41 \%$ \\
\hline $2.5 \mathrm{E}+05$ & 4.7304 & $6.05 \%$ & $5.0 \mathrm{E}+04$ & 1.3227 & $2.00 \%$ \\
\hline $5.0 \mathrm{E}+05$ & 5.5980 & $5.25 \%$ & $6.0 \mathrm{E}+04$ & 1.3826 & $5.62 \%$ \\
\hline $7.5 \mathrm{E}+05$ & 6.1775 & $4.38 \%$ & $7.0 \mathrm{E}+04$ & 1.4353 & $10.40 \%$ \\
\hline $1.0 \mathrm{E}+06$ & 6.6247 & $3.48 \%$ & $8.0 \mathrm{E}+04$ & 1.4827 & $11.89 \%$ \\
\hline $2.5 \mathrm{E}+06$ & 8.2765 & $0.78 \%$ & $9.0 \mathrm{E}+04$ & 1.5257 & $12.46 \%$ \\
\hline $5.0 \mathrm{E}+06$ & 9.7945 & $-1.16 \%$ & $1.0 \mathrm{E}+05$ & 1.5653 & $12.75 \%$ \\
\hline $7.5 \mathrm{E}+06$ & 10.8084 & $-3.06 \%$ & $2.0 \mathrm{E}+05$ & 1.8524 & $10.09 \%$ \\
\hline $1.0 \mathrm{E}+07$ & 11.5909 & $-4.42 \%$ & $4.0 \mathrm{E}+05$ & 2.1921 & $4.47 \%$ \\
\hline \multicolumn{2}{|c|}{$\begin{array}{l}\text { Average \% } \\
\text { Difference: }\end{array}$} & $8.52 \%$ & $5.0 \mathrm{E}+05$ & 2.3142 & $4.81 \%$ \\
\hline & & & $6.0 \mathrm{E}+05$ & 2.4190 & $7.68 \%$ \\
\hline & & & $8.0 \mathrm{E}+05$ & 2.5941 & $8.12 \%$ \\
\hline & & & $1.0 \mathrm{E}+06$ & 2.7387 & $7.49 \%$ \\
\hline & & & $2.0 \mathrm{E}+06$ & 3.2410 & $6.69 \%$ \\
\hline & & & $4.0 \mathrm{E}+06$ & 3.8354 & $5.79 \%$ \\
\hline & & & $5.0 \mathrm{E}+06$ & 4.0491 & $5.09 \%$ \\
\hline & & & $6.0 \mathrm{E}+06$ & 4.2324 & $6.22 \%$ \\
\hline & & & $8.0 \mathrm{E}+06$ & 4.5388 & $4.04 \%$ \\
\hline & & & $1.0 \mathrm{E}+07$ & 4.7917 & $4.06 \%$ \\
\hline & & & $\begin{array}{l}\text { Avera } \\
\text { Differ } \\
\end{array}$ & $\begin{array}{l}\text { ge } \% \\
\text { ence: }\end{array}$ & $5.45 \%$ \\
\hline
\end{tabular}


Table B.9: Curve-fitting to Nusselt number and percentage difference for $\theta_{0}=30^{\circ}$

\begin{tabular}{|c|c|c|c|c|c|c|}
\hline \multirow{2}{*}{ Gr } & \multicolumn{2}{|c|}{$\delta^{\prime}=1$} & \multicolumn{2}{|c|}{$\delta^{\prime}=0.8$} & \multicolumn{2}{|c|}{$\delta^{\prime}=0.5$} \\
\hline & $\mathrm{Nu}$ & $\begin{array}{c}\% \\
\text { Difference } \\
\end{array}$ & $\mathrm{Nu}$ & $\begin{array}{c}\% \\
\text { Difference } \\
\end{array}$ & $\mathrm{Nu}$ & $\begin{array}{c}\% \\
\text { Difference } \\
\end{array}$ \\
\hline $1.0 \mathrm{E}+03$ & 1.0000 & $0.24 \%$ & 1.0000 & $0.65 \%$ & 1.0899 & $-6.48 \%$ \\
\hline $2.5 \mathrm{E}+03$ & 1.0434 & $-1.02 \%$ & 1.1675 & $-6.88 \%$ & 1.3880 & $-10.39 \%$ \\
\hline $5.0 \mathrm{E}+03$ & 1.2754 & $-5.44 \%$ & 1.4165 & $-2.85 \%$ & 1.6666 & $-0.65 \%$ \\
\hline $7.5 E+03$ & 1.4344 & $-2.42 \%$ & 1.5861 & $0.79 \%$ & 1.8548 & $2.66 \%$ \\
\hline $1.0 \mathrm{E}+04$ & 1.5591 & $-0.24 \%$ & 1.7186 & $2.51 \%$ & 2.0011 & $3.86 \%$ \\
\hline $2.5 \mathrm{E}+04$ & 2.0331 & $3.21 \%$ & 2.2191 & $3.84 \%$ & 2.5486 & $4.32 \%$ \\
\hline $5.0 \mathrm{E}+04$ & 2.4853 & $3.24 \%$ & 2.6925 & $2.87 \%$ & 3.0601 & $3.20 \%$ \\
\hline $7.5 \mathrm{E}+04$ & 2.7952 & $2.78 \%$ & 3.0149 & $2.12 \%$ & 3.4057 & $2.35 \%$ \\
\hline $1.0 \mathrm{E}+05$ & 3.0381 & $2.36 \%$ & 3.2668 & $1.60 \%$ & 3.6744 & $1.71 \%$ \\
\hline $2.5 \mathrm{E}+05$ & 3.9619 & $1.05 \%$ & 4.2181 & $0.38 \%$ & 4.6795 & $-0.18 \%$ \\
\hline $5.0 \mathrm{E}+05$ & 4.8431 & $0.51 \%$ & 5.1179 & $0.03 \%$ & 5.6188 & $-1.22 \%$ \\
\hline $7.5 \mathrm{E}+05$ & 5.4468 & $0.37 \%$ & 5.7307 & $-0.01 \%$ & 6.2534 & $-1.66 \%$ \\
\hline $1.0 \mathrm{E}+06$ & 5.9202 & $0.30 \%$ & 6.2095 & $0.00 \%$ & 6.7467 & $-1.92 \%$ \\
\hline $2.5 \mathrm{E}+06$ & 7.7203 & $-0.05 \%$ & 8.0178 & $-0.10 \%$ & 8.5923 & $-2.60 \%$ \\
\hline $5.0 \mathrm{E}+06$ & 9.4375 & $-0.68 \%$ & 9.7281 & $-0.50 \%$ & 10.3170 & $-2.94 \%$ \\
\hline $7.5 \mathrm{E}+06$ & 10.6139 & $-2.04 \%$ & 10.8929 & $-0.88 \%$ & 11.4822 & $-3.02 \%$ \\
\hline $1.0 \mathrm{E}+07$ & 11.5365 & $-2.59 \%$ & 11.8030 & $-1.24 \%$ & 12.3879 & $-3.06 \%$ \\
\hline $\begin{array}{l}\text { Aver } \\
\text { Diffe }\end{array}$ & $\%$ & $1.68 \%$ & & $1.60 \%$ & & $3.07 \%$ \\
\hline
\end{tabular}


Table B-9: Continued

\begin{tabular}{|c|c|c|c|c|c|c|}
\hline \multirow[b]{2}{*}{$\mathbf{G r}$} & \multicolumn{2}{|c|}{$\delta^{\prime}=0.4$} & \multicolumn{2}{|c|}{$\delta^{\prime}=0.3$} & \multicolumn{2}{|c|}{$\delta^{\prime}=0.2$} \\
\hline & $\mathrm{Nu}$ & $\begin{array}{c}\% \\
\text { Difference } \\
\end{array}$ & $\mathrm{Nu}$ & \begin{tabular}{|c|}
$\%$ \\
Difference \\
\end{tabular} & $\mathrm{Nu}$ & $\begin{array}{c}\% \\
\text { Difference } \\
\end{array}$ \\
\hline $1.0 \mathrm{E}+03$ & 1.1275 & $-9.61 \%$ & 1.1917 & $-15.85 \%$ & 1.2997 & $-26.93 \%$ \\
\hline $2.5 \mathrm{E}+03$ & 1.4296 & $-11.00 \%$ & 1.5022 & $-18.35 \%$ & 1.6238 & $-32.34 \%$ \\
\hline $5.0 \mathrm{E}+03$ & 1.7108 & $-0.12 \%$ & 1.7898 & $-5.60 \%$ & 1.9218 & $-9.68 \%$ \\
\hline $7.5 \mathrm{E}+03$ & 1.9003 & $3.39 \%$ & 1.9829 & $-1.18 \%$ & 2.1208 & $-3.41 \%$ \\
\hline $1.0 \mathrm{E}+04$ & 2.0473 & $4.75 \%$ & 2.1325 & $0.69 \%$ & 2.2744 & $-0.85 \%$ \\
\hline $2.5 \mathrm{E}+04$ & 2.5959 & $5.80 \%$ & 2.6881 & $3.00 \%$ & 2.8417 & $2.84 \%$ \\
\hline $5.0 \mathrm{E}+04$ & 3.1065 & $5.11 \%$ & 3.2028 & $3.06 \%$ & 3.3631 & $3.77 \%$ \\
\hline $7.5 \mathrm{E}+04$ & 3.4506 & $4.46 \%$ & 3.5484 & $2.78 \%$ & 3.7114 & $3.83 \%$ \\
\hline $1.0 \mathrm{E}+05$ & 3.7176 & $3.93 \%$ & 3.8159 & $2.50 \%$ & 3.9802 & $3.63 \%$ \\
\hline $2.5 \mathrm{E}+05$ & 4.7136 & $2.16 \%$ & 4.8103 & $1.39 \%$ & 4.9730 & $-4.61 \%$ \\
\hline $5.0 \mathrm{E}+05$ & 5.6407 & $0.98 \%$ & 5.7312 & $0.42 \%$ & 5.8854 & $-4.55 \%$ \\
\hline $7.5 \mathrm{E}+05$ & 6.2655 & $0.43 \%$ & 6.3496 & $-0.13 \%$ & 6.4949 & $-4.43 \%$ \\
\hline $1.0 \mathrm{E}+06$ & 6.7503 & $0.07 \%$ & 6.8285 & $-0.46 \%$ & 6.9653 & $-4.27 \%$ \\
\hline $2.5 \mathrm{E}+06$ & 8.5589 & $-0.93 \%$ & 8.6078 & $-0.89 \%$ & 8.7027 & $-2.98 \%$ \\
\hline $5.0 \mathrm{E}+06$ & 10.2424 & $-1.64 \%$ & 10.2557 & $-0.58 \%$ & 10.2995 & $-1.90 \%$ \\
\hline $7.5 \mathrm{E}+06$ & 11.3768 & $-1.96 \%$ & 11.3624 & $-0.33 \%$ & 11.3661 & $-1.53 \%$ \\
\hline $1.0 \mathrm{E}+07$ & 12.2571 & $-2.08 \%$ & 12.2192 & $-0.14 \%$ & 12.1893 & $-1.36 \%$ \\
\hline $\begin{array}{l}\text { Aver } \\
\text { Diffe } \\
\end{array}$ & $\begin{array}{l}\% \\
\text { ce: }\end{array}$ & $3.44 \%$ & & $3.37 \%$ & & $6.64 \%$ \\
\hline
\end{tabular}


Table B-9: Continued

\begin{tabular}{c|c|c|c|c}
\hline \multirow{2}{*}{ Gr } & \multicolumn{2}{|c|}{$\delta^{\prime}=\mathbf{0 . 1}$} & \multicolumn{2}{c}{$\delta^{\prime}=\mathbf{0 . 0 1}$} \\
\cline { 2 - 5 } & $\mathbf{N u}$ & $\begin{array}{c}\% \\
\text { Difference }\end{array}$ & Nu & $\begin{array}{c}\% \\
\text { Difference }\end{array}$ \\
\hline $1.0 \mathrm{E}+03$ & 1.3775 & $-35.75 \%$ & 1.0000 & $0.11 \%$ \\
$2.5 \mathrm{E}+03$ & 1.6973 & $-52.10 \%$ & 1.1288 & $-12.10 \%$ \\
$5.0 \mathrm{E}+03$ & 1.9875 & $-13.03 \%$ & 1.3030 & $-27.28 \%$ \\
\hline $7.5 \mathrm{E}+03$ & 2.1799 & $-4.13 \%$ & 1.4170 & $-36.00 \%$ \\
\hline $1.0 \mathrm{E}+04$ & 2.3275 & $-0.95 \%$ & 1.5039 & $-36.84 \%$ \\
$2.5 \mathrm{E}+04$ & 2.8677 & $3.51 \%$ & 1.8180 & $2.43 \%$ \\
\hline $5.0 \mathrm{E}+04$ & 3.3582 & $5.45 \%$ & 2.0984 & $9.97 \%$ \\
\hline $7.5 \mathrm{E}+04$ & 3.6831 & $6.05 \%$ & 2.2821 & $10.63 \%$ \\
$1.0 \mathrm{E}+05$ & 3.9326 & $6.19 \%$ & 2.4221 & $10.56 \%$ \\
$2.5 \mathrm{E}+05$ & 4.8453 & $2.11 \%$ & 2.9278 & $6.51 \%$ \\
\hline $5.0 \mathrm{E}+05$ & 5.6741 & $1.37 \%$ & 3.3795 & $5.66 \%$ \\
\hline $7.5 \mathrm{E}+05$ & 6.2231 & $0.18 \%$ & 3.6753 & $1.70 \%$ \\
$1.0 \mathrm{E}+06$ & 6.6446 & $-0.49 \%$ & 3.9008 & $2.36 \%$ \\
\hline $2.5 \mathrm{E}+06$ & 8.1868 & $-1.06 \%$ & 4.7153 & $-2.01 \%$ \\
\hline $5.0 \mathrm{E}+06$ & 9.5870 & $-1.55 \%$ & 5.4426 & $-3.85 \%$ \\
\hline $7.5 \mathrm{E}+06$ & 10.5147 & $-2.30 \%$ & 5.9191 & $-6.35 \%$ \\
\hline $1.0 \mathrm{E}+07$ & 11.2268 & $-2.17 \%$ & 6.2822 & $-7.31 \%$ \\
\hline Average \% Difference: & $8.14 \%$ & & $10.69 \%$ \\
\hline & & & & \\
\hline & & & & \\
\hline
\end{tabular}




\section{References}

Alassar Rajai S., 2005, "Forced convection past an oblate spheroid at low and moderate Reynolds numbers”, Journal of Heat Transfer, vol. 127, pp. 1062-1070.

Anderson John D., Jr., 1995, "Computational Fluid Dynamics, the basics with applications", McGraw-Hill, Inc.

Bird R.B, Stewart W.E., Lightfoot E.N., 2001, “Transport phenomena”, second edition, Wiley, New York.

Bishop E.H., Kolflat R.S., Mack L.R. and Scanlan J.A., 1964, "Convective heat transfer between concentric spheres", Proceedings of the 1964 Heat Transfer and Fluid Mechanics Literature held at University of California, pp. 69-80.

Bishop E.H., Mack L.R. and Scanlan J.A., 1966, "Heat transfer by natural convection between concentric spheres", International Journal of heat and Fluid Flow, vol. 9 , pp. 649-662.

Caltagirone J.P., Combarnous M., Mojtabai A., 1980, "Natural convection between two concentric spheres: transition toward a multicellular flow", Numerical Heat Transfer, vol. 3, pp. 107-114.

Celik I., 1993, "Numerical uncertainty in fluid flow calculations: Needs for Future Research" ASME Journal of Fluids Engineering, vol. 115, pp. 194-195. 
Chen Chin-Lung and Cheng Chin-Hsaiang, 2002, "Buoyancy-induced flow and convective heat transfer in an inclined arc-shape enclosure", International Journal of Heat and Fluid Flow, vol. 23, pp. 823-830.

Chiu Cheng-Ping and Chen Wen-Ruey, 1996, "Transient natural convection heat transfer between concentric and vertically eccentric spheres", International Journal of Heat and Mass Transfer, vol. 39, no. 7, pp. 1439-1452.

Chow Y.L. and Yovanovich M.M., 1982, "The shape factor of the capacitance of a conductor", Journal of Applied Physics, vol. 53, no.12, pp. 8470-8475.

Curcija, D. and Goss W.P., 1993, "Two-dimensional natural convection over the isothermal indoor fenestration surface - finite element numerical solution", ASHRAE Transactions, vol. 99(1), pp. 274-287.

National Research Council of Canada (NRC), Institute for Research in Construction (IRC), 2007, "DayLight 1-2-3", http://www.daylight1-2-3.com/

FLUENT 6.2 Documentation, 2005, Fluent Incorporated, Evanston, Illinois.

Grag V.G. 1991, "Natural convection between concentric spheres”, International Journal of Heat and Mass Transfer, vol. 35, no. 8, pp. 1935-1945.

Gray, D.D and Giorgini, A. 1975, "The validity of the Boussinesq approximation for liquids and gases", International Journal of Heat and Mass Transfer, vol. 19, pp 545551.

Hassani A.V. and Hollands K.G.T., 1989, "On natural convection heat transfer from treedimensional bodies of arbitrary shape", International Journal of Heat and Fluid Flow, vol. 111, pp. 363-371. 
Hilpert R., 2002, "Chapter 7, External flow”, Introduction to Heat Transfer, Forth Edition, Editors: Incropera F.P. and DeWitt D.P., John Wiley \& Sons”, New York.

Jakob M., 1949, "Heat transfer”, vol. 1, John Wiley \& Sons, New York.

Laouadi A. and Atif M., 2001, 'Natural convection heat transfer within multi-layer domes", International Journal of Heat and Mass Transfer, vol. 44, pp. 1973-1981.

McGowan A.G., Desjarlais A.O. and Wright J.L., 1998, "Simulation and testing of pyramid and barrel vault skylights", ASHRAE Transactions: Symposia, pp. 832-844.

Melissari B. and Argyropoulos Stavros A., 2004, "The identification of transition convective regimes in liquid metals using a computational approach", An International Journal of Progress in Computational Fluid Dynamics, vol. 4, pp. 69-77.

Melissari B. and Argyropoulos Stavros A., 2005, "Development of a heat transfer dimensionless correlation for spheres immersed in wide range of Prandtl number fluids", International Journal of Heat and Mass transfer, vol. 48, pp. 433-4341.

Oosthuizen, P.H. and Naylor D., 1999, 'Introduction to convective heat transfer analysis', McGraw-Hill, New York.

Pop I. and Yan B., 1998, "Forced convection flow past a circular cylinder and sphere in a darcian fluid at large Peclet number", Int. Comm. Heat and Mass Transfer, vol.25, no.2, pp. 261-267.

Raithby G.D. and Hollands K.G.T., 1998, "Chapter 4, Natural convection”, in Hand-book of heat transfer, Editors: W.M. Rohsenow, J.P. Hartnett and Y.I. Cho, McGraw-Hill, New York. 
Raithby G.D. and Hollands K.G.T., 1975, “A general method of obtaining approximation solution to laminar and turbulent free convection problems", in Advances Heat Transfer, Editors: T.F. Irvine and J.P. Hartnett eds., vol. 11, pp. 266-315, Academic, New York.

Roache P.J., Ghia K.N. and White F.M., 1986, "Editorial policy statement on the control of numerical accuracy", ASME Journal of Fluids Engineering, vol. 108, pp. 2.

National Research Council of Canada (NRC), Institute for Research in Construction (IRC), 2007, "SkyVision", http://irc.nrc-cnrc.gc.ca/ie/lighting/daylight/skyvision e.html

Yin, S.H., Powe R.E., Scanlan J.A., and Bishop E.H., 1973, "Natural convection flow patterns in spherical annuli", International Journal of Heat and Mass Transfer, vol. 16, pp. 1785-1795.

Yovanovich M.M., 1987, “On the effect of shape, aspect ratio and orientation upon natural convection from isothermal bodies of complex shape", ASME Heat Transfer Division Winter Annual Meeting, Dec 13-18, vol. 82, pp. 121-129.

Versteeg H.K. and Malalasekera W., 1995, “An introduction to computational fluid dynamics the finite volume method", Prentice Hall.

Ziskind G., Zhao B., Katoshevski D. and Bar-Ziv E., 2001, "Experimental study of the forces associated with mixed convection from a heated sphere at small Reynolds and Grashof numbers in cross-flow", International Journal of Heat and Mass Transfer, vol. 44, pp. 4381-4389. 